\title{
Searching for molecular mechanisms of psychiatric diseases: examples from autism to rapid cycling
}

\section{Dissertation}

\author{
for the award of the degree \\ "Doctor rerum naturalium" \\ within the doctoral degree program \\ "Systems Neuroscience" \\ of the Georg-August-Universität Göttingen
}

submitted by

Artem Gurvich

born in Moscow

Göttingen 2014 


\section{Doctoral thesis committee}

Prof. Dr. Dr. Hannelore Ehrenreich (supervisor, first reviewer)

Clinical Neuroscience

Max Planck Institute of Experimental Medicine

Hermann-Rein-Straße 3

37075 Göttingen

Germany

Prof. Dr. André Fischer (second reviewer)

European Neuroscience Institute Göttingen

Grisebachstr. 5

37077 Göttingen

Germany

Prof. Dr. Nils Brose

Dept. of Molecular Neurobiology

Max Planck Institute

for Experimental Medicine

Hermann-Rein-Str. 3

37075 Göttingen

Germany

Date of submission of the thesis: 17.06.2014

Date of oral examination: 29.07.2014 


\section{Declaration}

I hereby declare that the thesis "Searching for molecular mechanisms of psychiatric diseases: examples from autism to rapid cycling" has been written independently and with no sources or aids other than those quoted.

Göttingen, June 17, 2014

Artem Gurvich 


\section{Acknowledgement}

I would like to express my deepest gratitude to my supervisor, Professor Hannelore Ehrenreich. I sincerely appreciate that you have granted me an opportunity to work in such an amazing place and to be surrounded by a unique atmosphere. You have been a tremendous mentor for me, providing me with patient guidance through every step of my work. Thank you for always being responsive and for giving all kinds of assistance immediately whenever it was needed. You have greatly contributed not only to my professional but also to my personal growth. I feel exceptionally lucky to have been able to work under your supervision.

I would also like to thank my thesis committee members, Professor André Fischer and Professor Nils Brose for constructive discussions during my progress report meetings.

I am particularly grateful to Professor Ralf Heinrich and Professor Martin Göpfert for introducing me to the Drosophila field, for providing me with all necessary equipment and expertise, and for carefully supervising my work with the flies.

I would like to thank all the present and past members of my research group. I wish to specifically thank Dr. Sergi Papiol for being my kind and patient guide in the field of genetics and more. I would like to offer my special thanks to Dr. Martin Begemann for being an amazing colleague and person of ready sympathy, generously sharing his professional expertise. Thank you to Anne Kästner for being a great colleague and for fun times during our trips to visit patients. I wish to thank Swetlana Sperling for guiding and assisting me with the lab procedures, and for being so reliable and kind to me. Thank you to Liane Dahm for continuous advice and help whenever it was needed. Thank you also to Kati Ostmeier for her substantial support regarding all the lab procedures. Thank you to Heather Sanders for correcting this manuscript with care. Giulia, you became my great and close friend and I am very thankful for your constant support and understanding.

Finally, I would like to thank my mother Elena Kazmina, my father Edward Gurvich and the whole Bazhenov family for helping me in all possible ways at every moment throughout these years, for constant encouragement, understanding, patience and love. It is of utmost importance for me to have your kind support. 


\section{Table of Contents}

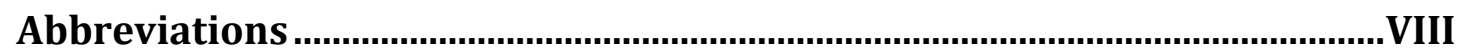

Preface

1. Introduction ............................................................................................. 1

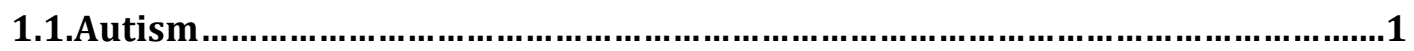

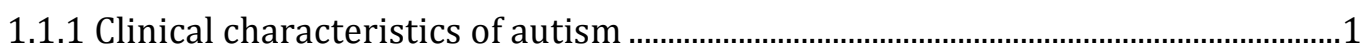

1.1.2 Genetic basis of autism: monogenetic cases ...........................................................2

1.1.3 Neuropsychiatric disorders as diseases of the synapse: emphasis on autism ...4

1.1.4. D. melanogaster as an animal model for ASD ...........................................................

1.2 Rapid cycling syndrome.........................................................................10

1.2.1 Clinical characteristics of rapid cycling bipolar disorder....................................10

1.2.2 Potential involvement of prostaglandins in bipolar disorder: preliminary evidence

1.2.3 Key players of the prostaglandin pathway ………………………………….........13

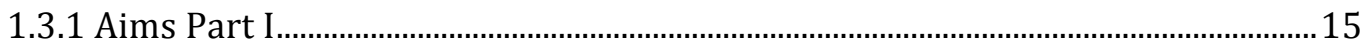

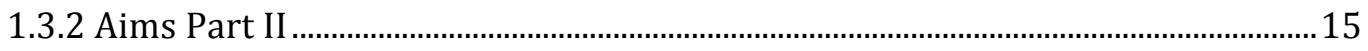

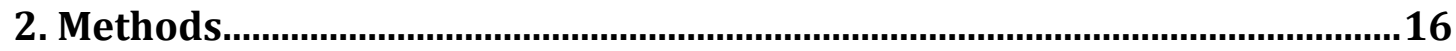

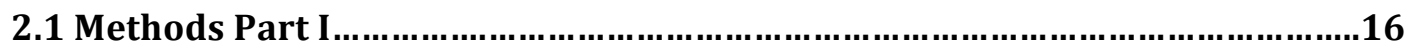

2.1.1 GRAS population and healthy controls ..................................................................16

2.1.2 Selection of the single nucleotide polymorphisms..................................................16

2.1.3 Genotyping procedures ......................................................................................... 17

2.1.5 Case - control association study ……………..........................................................18

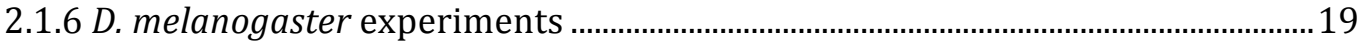

2.1.6.1 dnrxn1 -mutant animals ........................................................................................19

2.1.6.2 dnl2 -mutant animals ...........................................................................................19

2.1.6.3 Sound and video recordings ............................................................................. 19

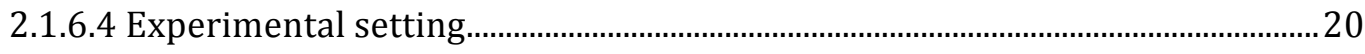

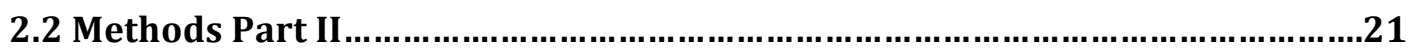

2.2.1 Patients with bipolar disorder and monopolar depression: cohort collection

2.2.2 Psychopathology rating............................................................................................. 21

2.2.3 Isolation of peripheral blood mononuclear cells (PBMCs) ....................................21

2.2 .4 qRT-PCR

2.2.5 Statistical analysis ............................................................................................... 22 


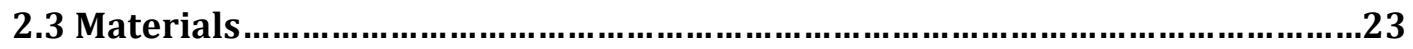

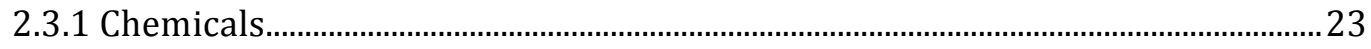

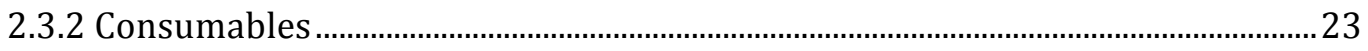

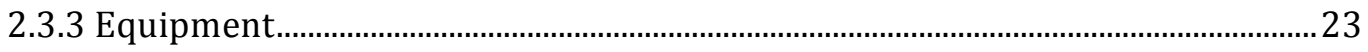

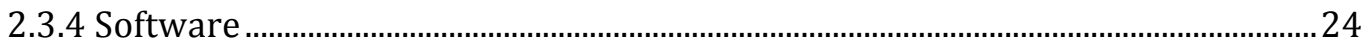

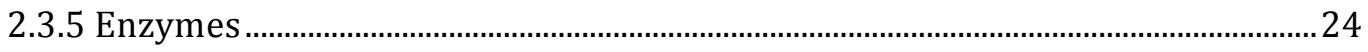

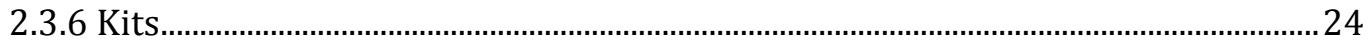

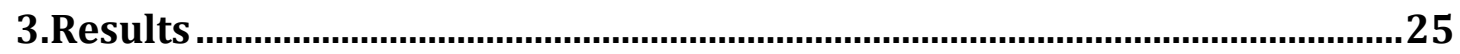

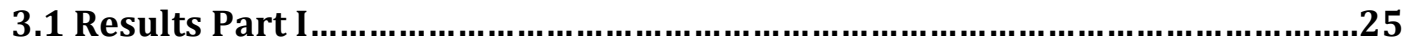

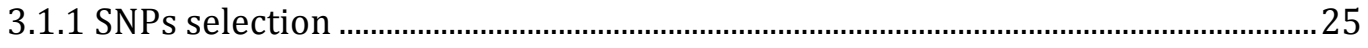

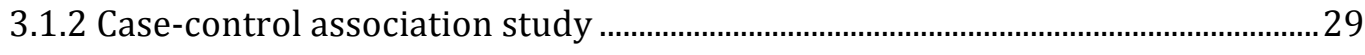

3.1.3 Screen testing of dnrxn and D. melanogaster ....................................................... 34

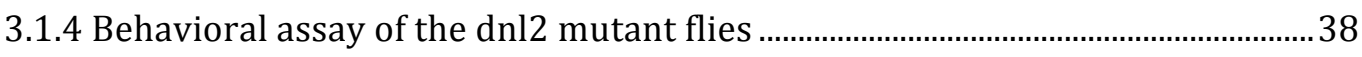

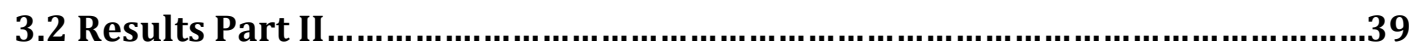

3.2.1 Demographic and clinical characteristics of four rapid cyclers...........................39

3.2.2 Bipolar and monopolar patients cohort description ............................................... 39

3.2.3 PTGDS, ARK1C3 and PTGS2/COX2 $2_{P A N}$ episode specific expression in rapid cyclers, patients with bipolar disorder and monopolar depression...............................39

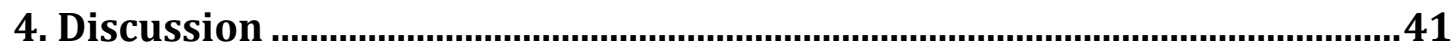

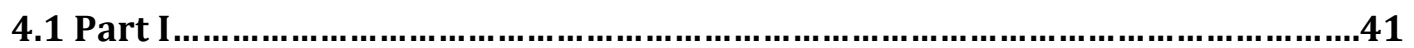

4.1.1 Synaptic genes and schizophrenia risk …………............................................... 41

4.1.2 D. melanogaster as a model of autism ...................................................................... 42

4.2 Part II: Prostaglandin related genes in rapid cycling bipolar disorder.........44

5. Outlook

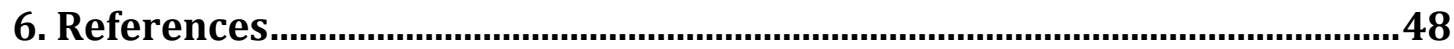

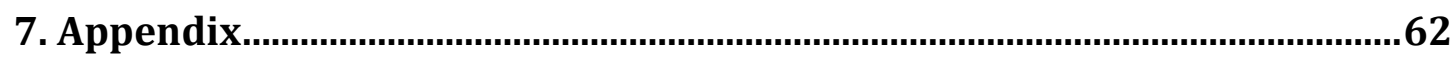

7.1 First author publication in press.....................................................62

7.2 Original publication: "Monogenic heritable autism gene neuroligin impacts

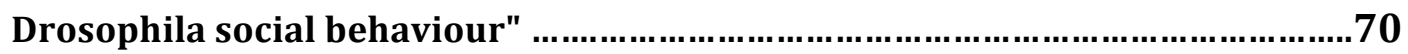

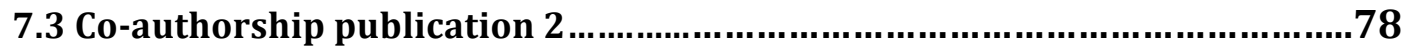

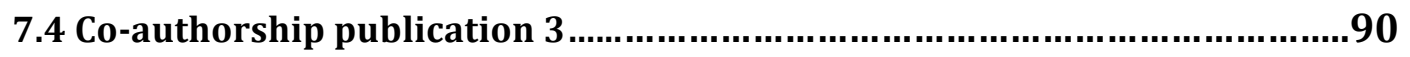

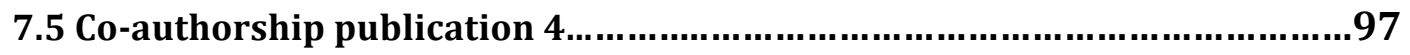




\section{Abbreviations}
AA
Arachidonic acid
ADI-R
Autism Diagnostic Interview, Revised
ADOS
Autism Diagnostic Observation Schedule,
AKR1C3
Aldo-keto reductase family 1 , member $\mathrm{C} 3$
$\operatorname{Arg}$
Arginine
ASD
Autism spectrum disorders
CASPR
Contactin-associated protein
CDNA
Complementary DNA
CIRL
Calcium-Independent Receptor for alpha-Latrotoxin
$\mathrm{CNV}$
Copy number variant
COX-1
Cyclooxygenase 1
COX-2
Cyclooxygenase-2
CPDA
Citrate Phosphate Dextrose Adenine
CS
Canton S
Cys
Cysteine
D. melanogaster
Drosophila melanogaster
DNA
Deoxyribonucleic acid
dnlgn2
Drosophila neuroligin 2
dnrxn1
Drosophila neurexin 1
dNTP
Deoxyribonucleotide
DSM-5
Diagnostic and Statistical Manual of Mental Disorders, 5th edition
EDTA Ethylenediaminetetraacetic acid
EGF
Epidermal growth factor, 15
EPSC
excitatory postsynaptic current, 15
GABA
Gamma-aminobutyric acid
GlyR
Glycerin receptor
GRAS
Göttingen Research Association for Schizophrenia
GWAS
Genome wide association study
HAMD
Hamilton rating scale for depression 


$\begin{array}{ll}\text { Hz } & \text { Herz } \\ \text { IPI } & \text { Interpulse interval } \\ \text { KB } & \text { Kilobase } \\ \text { KO } & \text { Knock out } \\ \text { LNS } & \text { Laminin/neurexin/sex hormone-binding globulin-domain } \\ \text { MAF } & \text { Minor allele frequency } \\ \text { mIPSC } & \text { Miniature inhibitory postsynaptic currents } \\ \text { mRNA } & \text { Messenger RNA } \\ \text { NAD+ } & \text { Nicotinamide adenine dinucleotide } \\ \text { NADPH } & \text { Nicotinamide adenine dinucleotide phosphate-oxidase } \\ \text { NLG4X } & \text { Neuroligin 4X } \\ \text { NLGN3 } & \text { Neuroligin 3 } \\ \text { NLGN4Y } & \text { Neuroligin 4Y } \\ \text { NLJ } & \text { Neuromuscular junction } \\ \text { NRXN1 } & \text { Neurexin 1 } \\ \text { P2RX7 } & \text { P2X purinoceptor 7 } \\ \text { PANSS } & \text { Positive and Negative Syndrome Scale } \\ \text { PBMC } & \text { Peripheral blood mononuclear cell } \\ \text { PCR } & \text { Polymerase chain reaction } \\ \text { PGAS } & \text { Phenotype-based genetic association study } \\ \text { PGD2 } & \text { Prostaglandin D2 } \\ \text { PGG2 } & \text { Prostaglandin G2 } \\ \text { PGH2 } & \text { Prostaglandin H2 } \\ \text { PGJ2 } & \text { Prostaglandin J2 } \\ \text { PPARy } & \text { Peroxisome Proliferator-Activated Receptor Activator } \\ \text { PSD } & \text { Post synaptic density protein } \\ \text { PTGDS } & \text { Prostaglandin D2 synthase } \\ \text { PTGS2 } & \text { Prostaglandin-endoperoxide synthase 2 } \\ \text { qRT-PCR } & \text { Real-time reverse-transcription quantitative polymerase } \\ \text { RNA } & \text { chain reaction } \\ \text { SD } & \text { Ribonucleic acid } \\ \text { SEM } & \\ & \text { Standard deviation } \\ \text { NArror of the mean }\end{array}$


SNP

TNF

UTR

VNC

YMRS

15PGDH
Single nucleotide polymorphism

Tumor necrosis factor

Untranslated region

Ventral nerve cord

Young mania rating scale

15-hydroxyprostaglandin dehydrogenase 


\section{Preface}

During my doctoral thesis I worked on several projects, aimed to investigate molecular mechanisms of neuropsychiatric disorders. These projects were:

1. The contribution of common variants of autism related synaptic genes to neuropsychiatric risk, as exemplified in schizophrenia.

Result: $\quad$ Common variants (Single Nucleotide Polymorphisms, SNPs) of synaptic genes NLGN3, NLG4X, NLGN4Y, SHANK3 and NRXN1 are not associated with global schizophrenia risk in the GRAS sample.

2. D. melanogaster, lacking dnrxn1 and dnlgn2, as plausible model for autism spectrum disorders.

Result: $\quad$ D. melanogaster dnrxn1 - KO flies demonstrated

locomotor impairment which did not allow testing of socially relevant readouts. Dnlgn2 - KO flies have normal motor activity and show impairment in social interaction.

3. Analysis of episode-specific gene expression of prostaglandin related genes in peripheral blood mononuclear cells of patients with rapid cycling bipolar disorder.

Result: $\quad$ Genes involved in the prostaglandin cascade show a phase specific differential expression pattern in rapid cycling patients.

Project 1 is a side project of a larger study on autism, spanning beyond the scope of this thesis. The results described here will later be incorporated in a publication, which is currently being prepared.

Project 2 has led to a publication, provided in the appendix. Additional experiments on dnrxn KO flies that I performed and analyzed, but that were not included in the publication, are presented here in detail. Other results are presented briefly here with reference to the appendix for details.

Project 3 is my first author publication, which is currently in press. Results and methods are shortly presented here with reference to the manuscript in the appendix for details.

Furthermore I have contributed as a coauthor to three other papers:

Hagemeyer et al, EMBO Molecular Medicine, 2012

Hammer et al, Molecular Psychiatry, 2013

Wojcik et al, Molecular Medicine, 2013. 


\section{Introduction}

\subsection{Autism}

\subsubsection{Clinical characteristics of autism}

Autism, first described in 1943 by Kanner (Kanner 1943), is listed under the category "Autism Spectrum Disorder" in the Diagnostic and Statistical Manual of Mental Disorders (DSM-5). The main features of Autism Spectrum Disorder (ASD) are: (A) persistent impairment of social interaction and communication; (B) restricted, repetitive patterns of behavior activities and interests; (C) persistence of the above mentioned symptoms since early childhood and (D) impairment of everyday living caused by the symptoms (AmericanPsychiatricAssociation 2013).

Autism is most often recognized by parents due to a delay in speech and language development at the mean age of 19 months (De Giacomo and Fombonne 1998). Some symptoms, which may include impaired orienting to name, looking at the faces of people around, imitative behavior and sharing of affect, may be recognized at earlier stages (Ozonoff, et al. 2008).

The symptom pattern in autistic individuals is presented in a broad spectrum with a considerable variability of symptom structure and severity across individuals (Geschwind 2009). A number of attempts have been made to define subgroups of patients based on various criteria, such as signs of early dysmorphogenesis (Miles, et al. 2005), patterns of social interaction (Castelloe and Dawson 1993; Wing and Gould 1979), intelligence quotient (Bartak and Rutter 1976) and neurocognitive profiles (Tager-Flusberg and Joseph 2003).

A number of studies have attempted to classify the clinical patterns using different diagnostic instruments, like ADI-R (Lord, et al. 1994) or ADOS (Lord, et al. 2000), or by applying various statistical approaches, such as principle component analysis, latent class analysis or factor mixture modeling (Boomsma, et al. 2008; Georgiades, et al. 2013; Georgiades, et al. 2011; Georgiades, et al. 2007; Kamp-Becker, et al. 2009; Munson, et al. 2008; 
Snow, et al. 2009; van Lang, et al. 2006). These studies represent an approach, where the distinctions in autism spectrum disorders are thought to be qualitative (Frazier, et al. 2010), whereas other researchers argue in favor of a dimensional approach, in which autistic traits are seen as a quantitative spectrum of traits (Constantino 2011). This is supported by the assessment of the distribution of autistic traits in the general population (Constantino and Todd 2003; Constantino and Todd 2005) and by the notion that some autistic behavioral traits have been observed in unaffected family members of autistic patients (Losh, et al. 2008; Murphy, et al. 2000; Piven, et al. 1997; Szatmari, et al. 2000). Interestingly, in addition to being seen in the general population, the autistic phenotype may also share common features with other psychiatric disorders, particularly schizophrenia. Thus, patients with ASD may suffer from paranoid (Craig, et al. 2004) and psychotic symptoms (Toal, et al. 2009), whereas patients of both disorders might show such symptoms as communication impairments (Bagner, et al. 2003; Condray, et al. 2002; DeLisi 2001) and social functioning deficits (Bellack, et al. 1990; Edwards, et al. 2002).

\subsubsection{Genetic basis of autism: monogenetic cases}

Prevalence of autism is estimated to be 62 affected individuals in 10,000 (Elsabbagh, et al. 2012). Autism spectrum disorders have a high concordance rate of $88 \%-95 \%$ for monozygotic twins, compared to the lower rate in dizygotic twins, ranging from 0\%-31\% (Bailey, et al. 1995; Rosenberg, et al. 2009; Taniai, et al. 2008). Heritability rates range from $80 \%-93 \%$ (Bailey, et al. 1995; Lichtenstein, et al. 2010). Various genetic mechanisms have been suggested to explain such a high genetic influence in the disease. Thus, genome wide association studies (GWAS) have revealed a number of single nucleotide polymorphisms (SNPs) and copy number variations (CNVs) associated with autism (Alarcon, et al. 2008; Maestrini, et al. 2010; Szatmari, et al. 2007; Weiss, et al. 2009). Rare and de novo CNVs were also identified as important genetic markers for ASD (Marshall, et al. 2008; Morrow, et al. 2008; Sebat, et al. 2007; Stefansson, et al. 2014). In genome wide linkage studies the regions $2 q, 7 q$ and $17 q, 15 q$ and $22 q$ have recurrently shown positive results for ASD (Freitag 2007; IMGSAC 2001; Kim, et al. 2008; 
Malhotra and Sebat 2012; Szatmari, et al. 2007). However, around 10\% of autistic cases may be explained by rare de novo mutations (Abrahams and Geschwind 2008; Weiss, et al. 2009). Among those traceable causes of these monogenic forms of ASD, a number of synaptic genes were identified.

Two genetic alterations in SHANK3 were shown to cause autism in a study by Durand et al.: a de novo $142 \mathrm{~kb}$ deletion of 22q13, mapping to exon 8 of SHANK3, and a $\mathrm{G}$ nucleotide insertion in exon 21 , which has later been shown by Arons et al. to lead to loss of function (Arons, et al. 2012; Durand, et al. 2007). A SHANK3 mutation in a third family, studied by Durand et al., a large $800 \mathrm{~kb}$ deletion in $22 \mathrm{q}$ was identified in a girl with autism, suffering from severe language impairment. Interestingly her brother suffering from Asperger syndrome with fluent speech but compromised social interaction, had a 22qter partial trisomy. Authors suggested an important role of fine gene dosage in the regulation of speech and communication abilities. Indeed, in further studies using a mouse model, mutations in Shank3 have caused a deficit in social interaction (Peca, et al. 2011), and a dose-related phenotype-genotype correlation has been observed in patients with deletions, including SHANK3 (Sarasua, et al. 2011). However, a mouse model overexpressing Shank3 shows a manic-like hyperactivity behavior (consistent with two human cases of SHANK3 duplication with attention deficit hyperactivity disorder (ADHD) and bipolar disorder reported in study), but no autism-like phenotype (Han, et al. 2013).

A study published by Jamain et al. has reported mutations in the homologous neuroligin genes, found in two unrelated families with siblings suffering from autism spectrum disorders (Jamain, et al. 2003). In one family a 1186insT NLGN4 frameshift mutation has been found in two siblings, one with typical autism and another with Asperger syndrome, but not in their unaffected brother. The mutation causes a stop codon and premature protein termination.

Another mutation, namely $1351 \mathrm{C} \rightarrow \mathrm{T}$ transition in $N L G N 3$, which results in the amino acid substitution Arg451 $\rightarrow$ Cys451, was found in both affected siblings of another family. Nonsynonymous mutations in NLGN4, namely $1597 \mathrm{~A} \rightarrow \mathrm{G}$ and $1253 \mathrm{del}(A G)$, have further been found in individuals with autism, as the 
studies of Laumonnier et al. and Pampanos et al. reported (Laumonnier, et al. 2004; Pampanos, et al. 2009). NLGN4X and NLGN3 are situated on the Xchromosome, in the regions $X p 22.3$ and $X q 13$ respectively, which have been affected in autistic cases (Auranen, et al. 2002; Gillberg 1998; Shao, et al. 2002; Shinawi, et al. 2009; Wentz, et al. 2013). The location of these genes on the X-chromosome may partially explain a male-to-female ratio of $4: 1$ among affected individuals (Fombonne 2003; Ritvo, et al. 1989). Moreover, mutations in another homologous sex-chromosome related gene, NLGN4Y situated on the $Y$ chromosome, was reported in an autistic case (Yan, et al. 2008). Further animal model studies, testing neuroligin deficient mice, have shown autism related phenotypes. Specifically, Nlgn3-knock out mice showed a deficit in social novelty and olfaction (Radyushkin, et al. 2009) and Nlgn4knock out mice showed a deficit in social behavior (Jamain, et al. 2008), while both null mutations resulted in reduced ultrasound vocalization and brain volume. Moreover, further tests of Nlgn 4 - knock out mice showed that these mice also show stereotyped repetitive behaviors detected by marble burying test, increased circling episodes in spontaneous homecage behavior and extensive grooming (El-Kordi, et al. 2013).

\subsubsection{Neuropsychiatric disorders as diseases of the synapse: emphasis on autism}

As discussed by Baudouin et al. (Baudouin 2014), the fact that such a wide range of underlying genetic patterns leads to a relatively convergent phenotype in ASD patients may be explained by the fact that the genetic markers identified in association and linkage studies might affect similar functional molecular pathways. Thus, the genetic findings concerning SHANK3, NLGN3, NLGN4X, and NLGN4 coding for post-synaptically localized proteins show that disturbance in synaptic functioning contributes to the complex autistic phenotype. Interestingly the NRXN1 gene, which codes for the binding partner of neuroligin proteins (Ichtchenko, et al. 1996), has also been associated with autism spectrum disorders (Kim, et al. 2008). 
Neurexins have initially been identified as receptors of $\alpha$-latrotoxin (a black widow venom, binding to presynaptic nerve terminals) as proteins, specifically expressed in the brain and present in excitatory and inhibitory neurons as shown by in-situ hybridization (Ullrich, et al. 1995; Ushkaryov, et al. 1992). Neurexin genes (I-III) each have two promoters, yielding transcripts for $\alpha$ - and $\beta$ - neurexins which, in combination with alternative splicing, results in thousands of isoforms (Missler and Sudhof 1998). Both longer $\alpha$-neurexins, containing five LNS and three EGF-like domains, and shorter $\beta$-neurexins, which lack those domains but have a laminin $G$ domain, can interact with postsynaptic neuroligins (Rudenko, et al. 1999; Ushkaryov, et al. 1994; Ushkaryov and Sudhof 1993). Neuroligins were isolated as binding partners of neurexins using an affinity matrix made from $\beta$-neurexins (Ichtchenko, et al. 1995; Ichtchenko, et al. 1996). Nguyen and Sudhof have shown a Ca2+ dependent binding of transfected cell lines expressing neuroligin-1 and neurexin $1 \beta$ (Nguyen and Sudhof 1997). Neuroligin-1 has been proven to localize in the postsynaptic membrane, where it extends into the synaptic cleft and co-localizes with glutamatergic synapses (Song, et al. 1999). Neuroligin-4 is situated (as studied in rodents) in inhibitory synapses within the retina, spinal cord, thalamus, colliculi and brainstem (Hoon, et al. 2011), similar to neuroligin-2 (Varoqueaux, et al. 2004). Studies in cultured neurons show that neuroligin-3 can be localized in inhibitory and in excitatory synapses (Budreck and Scheiffele 2007; Levinson, et al. 2010). Neuroligins contain a PDZbinding site on a C-terminal domain and have been shown to physiologically interact with PSD-95 and thus with glutamate receptors in excitatory synapses (Irie, et al. 1997). They also interact with collybistin in inhibitory synapses (Papadopoulos, et al. 2008) and with gephyrin (Graf, et al. 2004) connecting to GABA receptors. Further, direct yeast two-hybrid assays have shown that neuroligins interact with Shank3 (Meyer, et al. 2004), and a study in rodent neurons has shown that changes in levels of Shank3 provokes alterations in pre- and postsynaptic protein levels through neurexin-neuroligin signaling. Moreover, SHANK3 might have a functional connection to $N R X N 1$, due to its binding to latrophilins (CIRLs) which form NRXNs-like receptors to $\alpha$-latrotoxin (Tobaben, et al. 2000). 
As summarized by Krueger et al. (Krueger, et al. 2012), several lines of evidence show that the neuroligin-neurexin complex is involved in in-vitro synapse formation (Graf, et al. 2004; Scheiffele, et al. 2000), in-vivo synapse maturation and maintenance (Varoqueaux, et al. 2006), and its activitydependent validation (Chubykin, et al. 2007).

The link of these synaptic functions of the neuroligin-neurexin complex to an autistic phenotype has been made by performing animal model studies, such as a knock in mouse line, carrying an Arg451 $\rightarrow$ Cys451 mutation in neuroligin3 that was previously associated with ASD (Jamain, et al. 2003; Tabuchi, et al. 2007), and another line lacking Nlgn4 entirely (Jamain, et al. 2008). The Arg451 $\rightarrow$ Cys 451 knock in mutation, leading to a $90 \%$ decrease in neuroligin3 in mice, leads to an increase in inhibitory synaptic transmission and an increased number of GABAergic synapses, whereas this was not observed in mice with a complete knock out of Nlgn3 (Tabuchi, et al. 2007). This agrees with the hypothesis that an imbalance in inhibition and excitation may be one of the factors laying ground for an autistic phenotype, as reviewed by Rubenstein and Merzenich (Rubenstein and Merzenich 2003). Indeed, the prevalence of epileptiform electroencephalograms in autistic individuals has been estimated as high as 60\% (Spence and Schneider 2009). Furthermore, a study based on an optogenetic method showed that altered excitation and inhibition balance led to social behavior deficits in mice (Yizhar, et al. 2011).

Similarly, behavioral characterization of the knock in mice carrying the Arg451 $\rightarrow$ Cys451 mutation showed a decrease in social interaction in tests with a novel caged target mouse, and an increase in spatial learning and memory in the Morris water maze (Tabuchi, et al. 2007). This overlaps with the autistic phenotype in humans, since some individuals have normal to enhanced cognitive abilities in combination with impaired social interaction (AmericanPsychiatricAssociation 2013).

A knock out mouse lacking Nlgn4 shows reduced interest in conspecific mice and reduced ultrasound vocalization in contact with a female (Jamain, et al. 2008). Furthermore, the loss of Nlgn4 provokes a reduction in the number of glycerin receptors (GlyR) and leads to slower glycinergic miniature inhibitory postsynaptic currents (mIPSCs), as shown in a retina model (Hoon, et al. 
2011). Mice lacking Nrxn1 demonstrate a decrease in miniature excitatory postsynaptic current (EPSC) frequency, and decreased evoked excitatory synaptic strength (Etherton, et al. 2009), however, behavioral characterization of the Nrxn1 knock out model is somewhat inconsistent (Etherton, et al. 2009; Grayton, et al. 2013; Laarakker, et al. 2012).

Overall these data suggest an important role of synapse altered functionality in the autistic phenotype, where the neurexin-neuroligin-shank complex may be crucial for synapse stabilization and maturation, which occurs in the perinatal period, shortly before manifestation of the first signs of autism (Bourgeron 2009).

Synaptic alterations were also suggested to play an important role in schizophrenia, another disorder of the neuropsychiatric spectrum (Yin, et al. 2012). Genes encoding proteins involved in synaptic function were shown to play an etiological role in schizophrenia (Karlsgodt, et al. 2008; Stephan, et al. 2006). Post mortem studies revealed altered expression of synaptic proteins in the brain tissue of schizophrenic patients (Eastwood, et al. 2001; Harrison and Eastwood 1998; Knable, et al. 2004). Furthermore, genes involved in regulation of synaptic transmitter release and synaptic plasticity were shown to regulate disease symptomatology, in particular cognitive performance of schizophrenic patients (Begemann, et al. 2010; Grube, et al. 2011).

Interestingly, the Copy Number Variants (CNVs) of presynaptic NRXN1 were associated with both schizophrenia (Kirov, et al. 2008; Rujescu, et al. 2009; Walsh, et al. 2008) and autism (Kim, et al. 2008; Szatmari, et al. 2007). A genetic overlap based on rare structural variants can be seen between schizophrenia and autism (Sebat, et al. 2009). In a recent study, de novo mutations in genes, revealed by exome sequencing of $>600$ schizophrenia trios, were shown to overlap with de novo mutations identified in autism (Fromer, et al. 2014).

\subsubsection{D. melanogaster as an animal model for ASD}

Several Drosophila genes have been reported to be homologous to human neuroligins and neurexins, although none of them have a particular similarity to human orthologous genes. 
Drosophila neuroligin homolog CG31146, also referred to as Drosophila neuroligin 1 (dnlg1) has been identified in an unbiased mutagenesis screen for genes that influence neuromuscular structure in flies (Banovic, et al. 2010). The study was mainly focused on neuromuscular junctions (NLJ), which serve as a model for synaptic formation and functioning in Drosophila (Collins and DiAntonio 2007), and was able to isolate mutations in $d n \lg 1$, resulting in NMJs with a strongly reduced number of synaptic boutons. It was shown that $d n \lg 1$ is specifically expressed and functionally required at the postsynaptic side of the NMJ, which corresponds to the data about postsynaptic localization of neuroligins in humans, as described in chapter 1.1.3. The study of Banovic et al. was unable to identify any expression of this gene in the central nervous system (Banovic, et al. 2010).

A Drosophila gene named gliotactin, has been suggested as the most homologous to NLGN3 in humans (Gilbert, et al. 2001). Gliotactin is expressed in the glia, associated with the olfactory neurons from the maxillary palp and antennae (Gilbert, et al. 2001), which corresponds to the data in mice (Radyushkin, et al. 2009). However, despite the fact that the knock out embryos are morphologically normal, they are unable to hatch and were paralyzed (Zeev-Ben-Mordehai, et al. 2003). The latter obviously made behavioral tests of knockout flies impossible.

Another Drosophila gene CG13772, also referred to as Drosophila neuroligin 2 (dnlg2), codes for a protein which has a similar structure to vertebrate neuroligins: an N-terminal extracellular acetylcholinesterase-like domain, a single transmembrane region, and a C-terminal cytoplasmic region with a conserved PDZ binding motif (Sun, et al. 2011). Dnlg2 is expressed in the embryonic brain, ventral nerve cord and glutamatergic neuromuscular junction (NMJ) (Sun, et al. 2011). The same study has identified the strong co-localization of the dnlg2 with the Drosophila neurexin homolog, dnrxn, in the central nervous system and neuromuscular junction (NMJ), also outlining the important role of $d n l g 2$ in synaptic development. The expression of this gene in the CNS was widespread and uniform and was not preferentially localized with any specific neurotransmitter or neurohormone.

As mentioned previously, the expression analysis of dnlg2 high levels in the brain and ventral nerve cord (VNC) of third-instar larvae, and co-localization 
with dnrxn (Sun, The Journal of Neuroscience, 2011). The same colocalization has been seen in NMJ. This data goes along with the fact that human neuroligins and dnrxn form a complex in the synaptic region, performing important functions in synaptic transmission and differentiation as discussed in chapter 1.1.3. Dnrxn is reported to be homologous to vertebrae a-Neurexin (26\% amino acid identity with the rat a-neurexin 1) (Zeng, et al. 2007).

No additional neurexin-like genes or transcripts in Drosophila were revealed. A gene named Drosophila neurexin IV has been identified as an analog of the human Neurexin family gene CASPR (Baumgartner, S, Cell, 1996); however, as mentioned by Nguyen and Südhof (Nguyen and Sudhof 1997), it cannot be regarded as a human neurexin homolog distantly homologous to vertebrate neurexins, as it has a different domain structure and is expressed in glia but not neurons (Banerjee and Bhat 2007).

Dnrxn expression in the central nervous system is maintained at all stages of development (Zeng, et al. 2007). It was shown that the dnrx null mutants are viable, fertile, but have a reduced lifespan. The dnrx null mutants have fewer synaptic boutons, but more active zones per bouton (similar to dn/2 null mutants); however, in contrast to dn/2 null mutants, they show a decrease in transmitter release (Li, et al. 2007; Sun, et al. 2011).

While Sun et al. (Sun, et al. 2011) have focused on locomotor activity of different mutants lacking $d n r x$ and/or $d n / g 2$ (a homozygous mutant of $d n / 2$ that lacked one copy of $d n r x$, and a homozygous mutant for $d n r x$ that lacked one copy of dnl2), Zeng et al. (Zeng, et al. 2007) looked at associative learning in larvae of $d n r x$ mutant flies. The associative learning was reduced in hypomorphic mutants and more severely reduced in null mutant larvae. These findings provide some grounds to further test the central nervous system function in dnrx mutant flies.

Banovic et al. failed to detect any direct interactions between $d n r x$ and $d n \lg 1$ in neuromuscular junctions (Banovic, et al. 2010). However, dnrx and dnlgn1 mutants shared phenotypical similarities in abnormalities of synapse structure, while dnlgn1 mutations resulted in more severe disturbances.

Thus, given the fact Drosophila $d n r x$ and $d n l g 2$ mutants have not been previously tested for social interaction, performing such behavioral 
experiments may deliver first evidence for Drosophila to be used as a model organism for autism spectrum disorders.

One approach to investigate social behavior in Drosophila is to study their courtship behavior. The courtship behavior in Drosophila includes several steps (Greenspan and Ferveur 2000). The male orients towards the female, taps the female's abdomen and thorax with a foreleg, and extends the wing nearest to the female. Vibration of the wing generates the courtship song. Then comes the licking of the female's genitalia and an attempt to copulate. The courtship song is only produced by males and has been shown in playback experiments to reduce the female locomotion and to increase her receptivity (Gailey, et al. 1986). The song consists of a continuous oscillation known as the "sine song", followed by pulses, named the "pulse song", which are separated by so called interpulse intervals (IPI) (Tauber and Eberl 2003). The song can be characterized by various parameters (Gleason 2005), which are important in species recognition to prevent interbreeding.

In this study, several approaches using the readouts of the courtship behavior were used, which will be discussed in respective chapters and in the attached original publication in the appendix (Hahn, et al. 2013).

\subsection{Rapid cycling syndrome}

\subsubsection{Clinical characteristics of rapid cycling bipolar disorder}

The rapid cycling syndrome is a subtype of the bipolar disorder, which is characterized by the presence of manic phases and/or depressive phases. $A$ manic episode is described as a distinct period of abnormally elevated, expansive or irritable mood, causing impairment in social functioning, which is not attributed to any substance and is accompanied by at least three of the following symptoms: grandiosity, decreased need for sleep, pressure to keep talking, flight of ideas, increased distractibility, psychomotor agitation, and/or involvement in activities with potential painful consequences (AmericanPsychiatricAssociation 2013). Depressive episodes are characterized by the presence of at least five of the following symptoms: depressed mood, diminished interest in almost all activities, weight loss, 
hyper- or insomnia, stable psychomotor retardation or agitation, loss of energy, feeling of guilt, diminished concentration, and/or suicidal ideations; these symptoms are not due to any substance consumption and lead to impairment in social and occupational functioning (AmericanPsychiatricAssociation 2013).

Rapid cycling is listed in the DSM-5 as a specifier for bipolar disorder and it is mainly defined by the presence of at least four mood episodes in the previous twelve months that meet the criteria for a manic, hypomanic or major depressive episode (AmericanPsychiatricAssociation 2013). Faster switches in polarity, namely in the course of weeks to days (ultradian) and faster than once in 24 hours (ultra-rapid) have also been described (Kramlinger and Post 1996).

The term "rapid cycling" was first coined in a study aiming to identify the clinical features leading to unsuccessful treatment by lithium (Dunner and Fieve 1974). Indeed, rapid cycling syndrome was later shown to have a much higher clinical non-improvement rate than non-rapid cycling bipolar disorder and not only upon treatment with lithium (Tondo, et al. 2003).

This data, in combination with a relatively high prevalence of rapid cycling among bipolar patients (>12\%) (Kupka, et al. 2003), provides sufficient reasons for exploring the molecular mechanism underlying this syndrome.

\subsubsection{Potential involvement of prostaglandins in bipolar disorder:}

\section{preliminary evidence}

Begemann and colleagues published a case report, describing a female case with an extreme rapid cycling syndrome that has been monitored for over 20 years (Begemann, et al. 2008). The case report describes a female patient, born in 1945, with no prior medical illness and no evidence of neuropsychiatric illnesses in her family. In 1991, she became ill with rapid cycling syndrome of extreme severity and kept a diary of her illness, which was used to reconstruct 108 cycles over a 16 -year period. The time series suggests complex rhythms in periodicity with a mean total cycle length of $53 \pm$ $21 \mathrm{~d}$, switching within hours between manic (mean $28 \pm 14 \mathrm{~d}$ ) and depressed (mean $26 \pm 14$ d) episodes without normal intervals. 
To investigate possible underlying biological mechanisms, a strategy to identify genes differentially expressed in the manic and depressed phases has been applied. Firstly, eight blood samples were collected during two consecutive depressed and manic episodes on two consecutive days each. After screening by microarrays GeneChip Human Genome U133 Plus 2.0 (Affymetrix, Santa Clara, CA, USA), the data set was submitted to two bioinformatic processing steps. Any genes that differed between the two consecutive days within a particular episode were excluded (arbitrary daily variation). Further genes were excluded that were differentially expressed within the two depressed or within the two manic episodes (arbitrary monthly or inter-episode variation of the same mood state). The expression patterns of the remaining depressed and manic episode genes were subsequently compared. Genes found to be differentially expressed by microchip screening were confirmed by qRT-PCR in all samples (blood sampling was extended beyond the initial screening period, and regulated genes were again validated more than one year later).

Among other groups of genes, which have shown an episode specific expression pattern, the prostaglandin metabolism related genes PTGDS (lipocalin-type prostaglandin D synthetase) and AKR1C3 (prostaglandin D2 11-ketoreductase) were shown to have a higher expression in depressed episodes, and have been addressed further. A clinical experiment using a treatment approach was completed by applying a compassionate use of the cyclooxygenase inhibitor Celecoxib (Celebrex, Pfizer, $2 \times 200 \mathrm{mg}$ daily oral). A significant improvement in clinical symptomatology upon treatment with Celecoxib as measured with Hamilton Rating Scale for Depression (HAMD), Young Mania Rating Scale (YMRS) and Positive and Negative Syndrome Scale (PANSS) was reported.

Other studies also showed the effectiveness of COX2 inhibitors in the treatment of affective disorders. For example, a double blind add-on study by Müller et al. (Muller, et al. 2006) showed that Celecoxib was effective in reducing symptom severity in patients suffering from an acute depressive episode. This result was replicated in a study with a similar design three years later (Akhondzadeh, et al. 2009). Anti-inflammatory drugs from other classes, namely polyunsaturated fatty acids and anti-TNFalpha, were also effective in 
depression treatment, as recently reviewed by Fond et al. (Fond, et al. 2013). These treatment approaches were mostly derived from the idea of underlying inflammatory pathology in affective states (Leboyer, et al. 2012).

Interestingly, prostaglandin related genes have previously been shown to play an important role in hibernation. Specifically, Prostaglandin D2 $\left(\mathrm{PGD}_{2}\right)$ shows increased expression levels in the brain during the torpor phase in chipmunks (Takahata, et al. 1996). Also, the mRNA levels of Prostaglandin D2 Synthase (PTGDS) declines significantly in the hypothalamus of ground squirrels across the hibernation cycle (O'Hara, et al. 1999), whereas intracerebral injections of $\mathrm{PGD}_{2}$ promotes sleep in rats and monkeys (Hayaishi 1999; Onoe, et al. 1988). These findings allow parallels to be drawn between rapid cycling syndrome and hibernation (Begemann, et al. 2008).

\subsubsection{Key players of the prostaglandin pathway}

One key player in the prostaglandin pathway, which has been used as a clinical target for decades, is cyclooxygenase (COX), also named Prostaglandin-endoperoxide synthase (PTGS), which catalyzes the synthesis

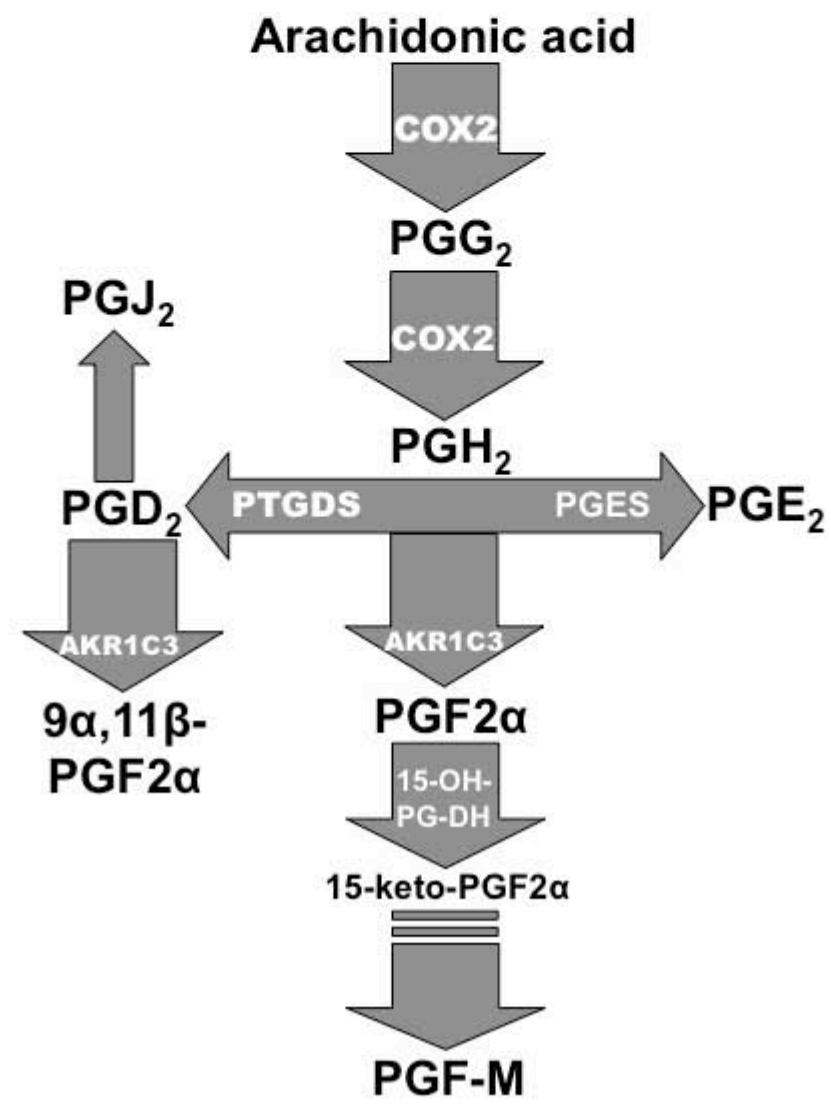

Figure 1 Key players of the Prostaglandin Pathway, modified from Gurvich et al, in 
of Prostaglandin $\mathrm{G}_{2}\left(\mathrm{PGG}_{2}\right)$ and Prostaglandin $\mathrm{H}_{2}\left(\mathrm{PGH}_{2}\right)$ by arachidonic acid (AA) (DeWitt and Smith 1988).

The COX gene has two isoforms, namely COX-1 and COX-2, which share a great structural and biochemical similarity, and both have arachidonic acid as a substrate; however, COX-1 activity is physiological, whereas COX-2 activity is mostly induced and pathological (Vane, et al. 1998). COX-2 is expressed also constitutively in the brain, specifically in the forebrain, and is enriched in the cortex, in the hippocampus in neuronal cells (Yamagata, et al. 1993), in microglia and in endothelial cells (Laflamme, et al. 1999). The two structurally and functionally interconnected sites (the cyclooxygenase site and peroxidase site) of the cOX protein catalyze two consecutive reactions: first is the cyclooxygenase reaction, in which arachidonate and two $\mathrm{O}_{2}$ molecules are converted to $P \mathrm{PG}_{2}$, and then the peroxidase reaction occurs, in which $P \mathrm{GG}_{2}$ is converted to $\mathrm{PGH}_{2}$ (Smith, et al. 2000). The Cyclooxygenase 2 coding gene has two isoforms, referred to as PTGS2, which has been identified in brain tissue (Knott, et al. 2000), and PTGDS2a, so far only identified in thrombocytes (Censarek, et al. 2004).

Additionally, several metabolic pathways are possible. $\mathrm{PGH}_{2}$ can be converted to Prostaglandin $D_{2}\left(P G D_{2}\right)$ in a reaction catalyzed by Prostaglandin $D_{2}$ synthase (PTGDS) (Urade and Hayaishi 2000).

$P G D_{2}$ is then non-enzymatically converted to Prostaglandin $J_{2}\left(P G J_{2}\right)$ and then to $15 \Delta-\mathrm{PGJ}_{2}$ (Desmond, et al. 2003), which activates Peroxisome Proliferator-Activated Receptor Activator $\gamma$ (PPAR $\gamma$ ), a molecule important for the inhibition of pro-inflammatory signals (Dave and Amin 2013). In the presence of Aldo-keto reductase family 1 member C3 (AKR1C3), however, another pathway is activated. In this pathway, the protein AKR1C3, also referred to as type $517 \beta$ - hydroxysteroid dehydrogenase (17 $\beta-H S D)$ due to its involvement in the steroid hormone metabolism (Lin, et al. 1997) and as prostaglandin F synthase (Matsuura, et al. 1998), exhibits two type of activities in the prostaglandin pathway. Firstly, $\mathrm{PGH}_{2}$ 9,11-endoperoxide reductase activity catalyzes the formation of Prostaglandin $F 2$ alpha $\left(P F_{2} \alpha\right)$ directly from $\mathrm{PGH}_{2}$, and secondly, $\mathrm{PGD}_{2}$ 11-ketoreductase activity, which in the presence of NADPH catalyzes the formation of $9 \alpha, 11 \beta-\mathrm{PGF}_{2}$ from $\mathrm{PGD}_{2}$ 
(Komoto, et al. 2004; Liston and Roberts 1985; Watanabe, et al. 1986). $\mathrm{PGH}_{2}$ can also be converted to Prostaglandin $E_{2}\left(P E_{2}\right)$ by Prostaglandin $E$ synthase (PGES) (Nakanishi and Rosenberg 2013). Finally, $\mathrm{PGH}_{2}$ can be processed by NAD+-linked 15-hydroxyprostaglandin dehydrogenase (15PGDH), which leads to the formation of low biologically active 15-keto metabolites, which, taking into consideration the reciprocal interaction of 15PGDH and COX-2, allows 15PGDH to be regarded as the enzyme responsible for the inactivation of prostaglandins (Tai, et al. 2006).

\subsubsection{Aims Part I}

The aim of the project related to autism was to (a) investigate the plausible contribution of normal variation in autism related synaptic genes as risk factors in a schizophrenic population, applying a case-control design, and (b) to further explore the contribution of these genes to social behavior, suggesting Drosophila melanogaster as an animal model.

\subsubsection{Aims Part II}

The aim in Part II of the study was to further investigate the role of prostaglandin related genes in the pathogenesis of the rapid cycling syndrome. For this purpose it was set to explore (a) whether the results published earlier by our group can be replicated in other rapid cyclers, (b) whether another gene involved in the prostaglandin metabolism of COX2 will also show phase dependent changes in mRNA levels, and (c) whether such changes can also be observed in a sample of patients suffering from nonrapid cycling bipolar disorder and/or monopolar depression. 


\section{Methods}

\subsection{Methods Part I}

\subsubsection{GRAS population and healthy controls}

The Göttingen Research Association for Schizophrenia patient data collection has been extensively described elsewhere (Ribbe, et al. 2010). Briefly, in the time period between 2005 and 2008, a total of 1071 schizophrenic patients were investigated by a travelling team all across Germany. For 1041 patients $(66.7 \% \mathrm{men} / 33.3 \%$ women; mean age $39.54 \pm 12.55)$, the diagnosis of schizophrenia or schizoaffective disorder was confirmed by a careful re-check of diagnostic criteria.

Healthy control subjects, who gave written informed consent, were voluntary blood donors and were recruited by the Department of Transfusion Medicine at the Georg-August-University of Göttingen according to national guidelines for blood donation. They fulfilled health criteria, confirmed by a pre-donation screening process containing standardized questionnaires and interviews, as well as hemoglobin, blood pressure, pulse, and body temperature determinations. Blood samples from a total of 2265 subjects were obtained $(\mathrm{m} / \mathrm{f}=1303 / 962)$ with the mean age of $33.8 \pm 12.2$ years, with a range from 18 to 69 years. Participation as healthy controls for the GRAS sample was anonymous, with information restricted to age, gender, blood donor health state and ethnicity.

\subsubsection{Selection of the single nucleotide polymorphisms}

The selection of the single nucleotide polymorphisms (SNPs) was first performed by making an extensive database/ in silico analysis using the openaccess databases: The National Center for Biotechnology Information (NCBI)(Sherry, et al. 2001), The University of California Santa Cruz genome 
browser (UCSC)(Kent, et al. 2002), Ensembl genome browser (Flicek, et al. 2013), and data from the Hapmap project (HapMapConsortium. 2003).

\section{Criteria for selection}

The SNPs for the genes NLGN3, NLGN4X, NLGN4Y, NRNX1, and SHANK3 were selected to be of a relatively equal distance from each other across the gene. The linkage disequilibrium - the non-random association of alleles was taken into account, so that each SNP would at most report by itself for the corresponding region, not serving as a proxy for another selected marker.

To make sure that the study has enough power to detect the plausible effects, the minor allele frequencies (MAF) were selected so that most of the selected SNPs had a MAF of around 0.5 and only few of them had the MAF around 0.15 .

\subsubsection{Genotyping procedures}

DNA from whole blood was isolated using the JETQUICK Blood \& Cell Culture DNA Spin Kit (Genomed GmbH, Löhne, Germany). Two vials EDTA whole blood (S-Monovette ${ }^{\circledR}$ EDTA K2 Gel, Sarstedt, Germany), (ca 2,7 ml) were poured into one Falcon tube and mixed with 150 RNase A (Roche Diagnostics $\mathrm{GmbH}$, Mannheim, Germany). Following the addition of $250 \mu \mathrm{l}$ of Protease, the contents were mixed vigorously and $5 \mathrm{ml}$ of $\mathrm{K} 1$ Buffer was added. The tubes were then incubated for $10 \mathrm{~min}$ at $70{ }^{\circ} \mathrm{C} ; 5 \mathrm{ml}$ of ethanol was added, mixed vigorously and the solution was put through a JETQUICK Maxi-spin Column. The column was centrifuged for $3 \mathrm{~min}$ at $2000 \mathrm{xg}$. The flow-through was discarded, $10 \mathrm{ml}$ of $\mathrm{KX}$ Buffer was introduced to the column and the column was centrifuged for $5 \mathrm{~min}$ at $4000 \mathrm{xg}$. The flow-through was discarded, $10 \mathrm{ml}$ of $\mathrm{K} 2$ Buffer was applied and the column was again centrifuged for $5 \mathrm{~min}$ at $4000 \mathrm{xg}$. Once more, the flow-through was discarded and the column was centrifuged for $17 \mathrm{~min}$ at $4000 \mathrm{xg}$. The column was put

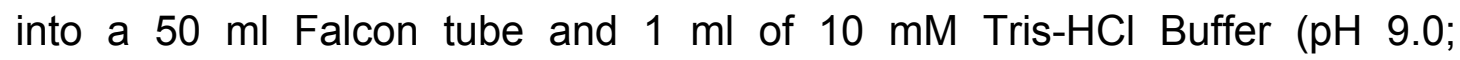


preheated to $70^{\circ} \mathrm{C}$ ) was added. This mixture was incubated for $5 \mathrm{~min}$ at room temperature and centrifuged for $3 \mathrm{~min}$ at $4000 \mathrm{xg}$. The flow-through was collected into Eppendorf tubes.

Genotyping was performed with simple probes, ordered from TIB Molbiol, Berlin, Germany. Simple probes for overall 22 SNPs in the NLGN3, NLGN4X, NLGN4Y, NRNX1, and SHANK3 were ordered.

First a test run with a limited sample set of human DNA and a water negative control was performed for each SNP probe to determine the best master mix solution:

\begin{tabular}{|c|c|c|c|c|c|}
\hline Master mix & Solution & Reagent Mix & Genotyping Mix & $\mathrm{MgCl}_{2}$ & Water \\
\hline 1 & $1: 10,3 m M M g C L_{2}$ & $0,5 \mu l$ & $1 \mu l$ & $0,6 \mu l$ & $5,9 \mu l$ \\
\hline 2 & $1: 10,1,5 \mathrm{mM} \mathrm{MgCL} 2$ & $0,5 \mu l$ & $1 \mu l$ & - & $6,5 \mu l$ \\
\hline 3 & $1: 20,3 m M M g C L_{2}$ & $0,5 \mu l$ & $0,5 \mu l$ & $7,2 \mu l$ & $6,7 \mu l$ \\
\hline 4 & $1: 10,2,5 \mathrm{mM} \mathrm{MgCL} 2$ & $0,5 \mu l$ & $0,5 \mu l$ & $2,4 \mu l$ & $6,7 \mu l$ \\
\hline
\end{tabular}

The test runs with $2 \mu$ l of DNA per sample and $8 \mu$ of the described master mixes were performed in the Roche LC480 Light Cycler (Roche Diagnostics $\mathrm{GmbH}$, Mannheim, Germany). The master mix for each SNP was selected according to the best Melting Curves profile as compared to those provided by TIB Molbiol. Furthermore, the genotyping of all subjects and healthy controls was performed using the best master mix in the Multiwell plate 384 (Roche Diagnostics $\mathrm{GmbH}$, Mannheim, Germany).

The Melting curves were analyzed using the LightCycler® 480 Software, Version 1.5. The Tm-calling and the curve shape for each patient was checked and in case of unusual curve shape or shifted peak melting temperature (as compared to the profile provided by TIB Molbiol for each probe), the PCR run was repeated.

\subsubsection{Case - control association study}

The case - control study was performed using the SPSS Statistics 17.0 for Mac (BM Deutschland GmbH, Ehningen, Germany). The Pearson Chi-square 
test was used to assess if the genotype frequencies were different between the case and the control group.

\subsubsection{Drosophila melanogaster experiments}

\subsubsection{1 dnrxn1 -mutant animals}

Originally two populations of balanced flies were obtained: DfExcel6191/Tm6b and $n r x-1241$ / Tm3c. Tubby pupae of DfExcel6191/Tm6b were collected and virgin females were separated. This was done based on the following criteria: female virgin flies have larger abdomens, the abdomen is very tender to the touch and white colored, and finally, females with a greenish spot on their dorsal part (meconium) are virgins. They were crossed against $n r x-1241$ / Tm3c males. The progeny of nrx-1241/DfExcel6191 was collected, first selecting the non-Tubby pupae and then the fetched flies lacking the Serrate maker. Virgin females were collected. Males and females were separated from each other in groups until the experiments.

\subsubsection{2 dn/2 -mutant animals}

dn/2-deficient mutant lines $\left(d n / 2^{K O 17}, d n / 2^{K O 70}\right)$, generated by targeted knock out of the dn/2 genomic locus (Sun, et al. 2011), were obtained. Virgin 7 days old decapitated wild type females were used as courtship targets and males were between 7 and 12 days of age and socially naïve. All recordings were performed at temperature $\left(20-22^{\circ} \mathrm{C}\right)$. Results reported are taken from $d n / 2^{K O 17}$ flies, whereas trial tests with $\mathrm{dnl} 2^{\mathrm{KO} 0}$ flies have shown similar, though sometimes weaker effects in acoustic communication effects. A detailed description is presented in the original publication (Hahn, et al. 2013), see Appendix.

\subsubsection{Sound and video recordings}

Acoustic signals were recorded in a sound proof chamber with a microphone (Bruel\&Kjaer Type 4165), and then amplified (Bruel\&Kjaer Type 2619 and 5935) and directly digitized. The software Audacity 1.3 .12 beta (http://audacity.sourceforge.net) was used for data acquisition and analysis. 
The total duration of sine and pulse songs was determined between the 1st and 6th minutes of the recording period. From each recorded male (where possible) three representative song sequences, that contained both pulse and sine song, were selected for the following analysis. Frequency spectra of pulse songs and sine songs were determined by Fourier transformation with 2048 Hanning window. The dominant frequency component was determined from these spectra. Interpulse intervals were determined for pulse song periods.

Video recording using a Logitec Web-Camera were done simultaneously with the sound recording.

The experimenter was blinded to genotypes during data acquisition and analysis.

\subsubsection{Experimental setting}

For $n r n x-1$ mutant flies, courtship songs were recorded during a 10-minute interaction between one 4-7 day old mutant (nrx-1241/DfExcel6191) male or one control (Canton-S) male and one intact virgin 4-7 day old wild type Canton-S female at room temperature. Additionally, 4-7 day old Canton $S$ males were tested during interaction with 4-7 day old virgin mutant (nrx1241/DfExce/6191) females under the same conditions. Flies were tested in two sets, on separate days, at the same time of the day (around mid-day). The experimenter was blind to the genotypes while performing the experiments and analyzing the data.

The experimental setting used in testing the dn/2 mutant flies is described in the original publication (Hahn, et al. 2013) in the appendix. Here is a short summary.

In an inter-individual distance assay ten mature flies of the same genotype were put in a featureless arena. After an exploration period, their individual positions were recorded, analyzed by ivTools software suite (developed by Lindemann \& Braun, https://opensource.cit-ec.de/projects/ivtools) and MatLab (Mathworks inc.) was used to calculate the median distance of each fly from all other flies in the assay. The competitive courtship assay was performed 
with a decapitated female and two males in a round arena; videos were recorded. Courtship and agonistic interactions during the 1st, 4th, 7th, 10th and 13th minute were scored through analysis of individual frames by an observer blind to the genotype of the flies. To assess the circadian locomotor activity, the flies were put into a glass tube $(3 \mathrm{~mm}$ in diameter and $7 \mathrm{~mm}$ in length) sealed with food at one end and with a gas permeable cap on the other. The tube was put into a special incubator (Tritech Research, CircKinetics). Midline crossings of individual flies were counted over 96 hours.

\subsection{Methods Part II}

\subsubsection{Patients with bipolar disorder and monopolar depression:}

\section{cohort collection}

The detailed description of Methods Part II is presented in the manuscript (Gurvich et al in press) attached in the Appendix. Here a short summary.

The collection of data and samples has been approved by the ethical committee of the Georg-August-University of Göttingen. All patients have signed an informed consent. The recruitment took place between 2010 and 2013. Four rapid cycling patients ( 3 males and 1 female) were included in the study, as well as 43 patients with non-rapid cycling bipolar affective disorder and 97 monopolar depressed patients. All patients kept their preexisting medication and no systematic intervention was undertaken in this study.

\subsubsection{Psychopathology rating}

Psychopathology was rated using standard scores: Hamilton Depression Rating Scale (HAMD) (Hamilton 1967), Beck Depression Inventory (BDI) (Beck, et al. 1961), Yong Mania Rating Scale (YMRS) (Young, et al. 1978) and Positive and Negative Syndrome Scale (PANSS) (Kay, et al. 1987).

\subsubsection{Isolation of peripheral blood mononuclear cells (PBMCs)}

Blood samples, obtained via phlebotomy into CPDA vials (Citrate Phosphate Dextrose Adenine, Sarstedt, Germany) underwent the standard Ficoll-Paque Plus isolation procedure (GE Healthcare, Munich, Germany). RNA isolation was performed using the miRNeasy Mini Kit (Qiagen, Hilden, Germany). The 
cDNA was transcribed from $1 \mu \mathrm{g}$ of total RNA with a mixture of oligo-dT- and hexamer primers, dNTPs, DTT and 200U SuperScriptlll (Life Technologies $\mathrm{GmbH}$, Darmstadt, Germany). The mixture was incubated for $10 \mathrm{~min}$ at $25^{\circ} \mathrm{C}$, $45 \mathrm{~min}$ at $50^{\circ} \mathrm{C}$ and $45 \mathrm{~min}$ at $55^{\circ} \mathrm{C}$.

\subsection{4 qRT-PCR}

For the qRT-PCR, the cDNA was diluted $1: 25$ in a $10 \mu l$ reaction mix, containing $5 \mu$ I SYBR Green (Life Technologies GmbH, Darmstadt, Germany) and $1 \mathrm{pmol}$ of each primer.

The following primer sequences were used:

PTGDS: 5' CGGCTCCTACAGCTACCG 3' (forward)

5' CAGCGCGTACTGGTCGTA 3' (reverse)

AKR1C3: 5' CATTGGGGTGTCAAACTTCA 3' (forward)

5' CCGGTTGAAATACGGATGAC 3' (reverse)

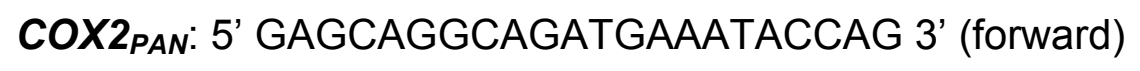

5' GTTGGAAGCACTCTATGGTGACA 3' (reverse)

PTGS2: 5' GATCCCCAGGGCTCAAACAT 3' (forward)

5' TCATATTTACGGTGAAACTCTGG 3' (reverse)

P2RX7: 5' AGGAAGAAGTGCGAGTCCAT 3' (forward)

5' CTGCTGGTTCACCATCCTAA 3' (reverse)

The samples were run with technical triplicates on the LightCycler480 system (Roche Diagnostics GmbH, Mannheim, Germany).

A detailed procedure description is provided in the manuscript attached in the Appendix.

\subsubsection{Statistical analysis}

All numerical results are presented as mean $\pm S D$ in the text and mean $\pm S E M$ in the figures. Student $t$ test (two-tailed or one-tailed if applicable) was performed using GraphPad Prism version 5.04 for Windows, GraphPad Software, San Diego California USA. 
2.3 Materials

\subsubsection{Chemicals}

\section{Product}

Chloroform

Ethanol 99,8\%

Ficoll-Paque PLUS

Power SYBR Green

\section{Supplier}

Merck KGaA, Darmstadt (Germany)

Sigma-Aldrich, Steinheim (Germany)

GE Healthcare Munich (Germany)

Life Technologies GmbH, Darmstadt (Germany)

\section{Product number}

102445

603002005

17144003

4367659

\subsubsection{Consumables}

\section{Product}

S-Monovette CPDA

Microwell plate 96

Multiwell plate 384

\section{Supplier}

SARSTEDT AG \& Co., Nümbrecht (Germany)

Thermo Fisher Scientific, Roskilde (Denmark)

Roche Diagnostics, Mannheim (Germany)
Product number

051167001

269620

04729749001

\subsubsection{Equipment}

\section{Product}

EpMotion robot 5075

Light Cycler 480

Water purification system arium 611

\section{Centrifuges}

Megafuge 16R

Megafuge 3.0

Mini Spin Plus

Biofuge Haemo

\section{Supplier}

Eppendorf AG, Hamburg (Germany)

Roche Diagnostics GmbH, Mannheim (Germany)

Sartorius, Göttingen (Germany)

Thermo Fisher Scientific, Bonn (Germany)

Thermo Fisher Scientific, Bonn (Germany)

Eppendorf AG, Hamburg (Germany)

Thermo Fisher Scientific, Bonn (Germany) 


\subsubsection{Software}

\section{Product}

Audacity 1.3 .13 beta

GraphPad Prism 5

LightCycler ${ }^{\circledast} 480$ Software, Version 1.5 SPSS

\section{Supplier}

http://audacity.sourceforge.net/

GraphPad Software Inc., La Jolla (USA)

Roche Diagnostics $\mathrm{GmbH}$, Mannheim (Germany)

IBM Deutschland GmbH, Ehningen (Germany)

\subsubsection{Enzymes}

\section{Product}

RNase A

SuperScript III

\section{Supplier}

Roche Diagnostics GmbH, Mannheim (Germany)

Life Technologies GmbH, Darmstadt (Germany)

\section{Product number}

10109169001

18080-044

\subsubsection{Kits}

\section{Product}

\section{Supplier}

Product number

miRNeasy Mini Kit

Qiagen, Hilden (Germany)

217004

JETQUICK Blood \& Cell Culture DNA Spin Kit

Genomed GmbH, Löhne, 440050

(Germany) 


\section{Results}

\subsection{Results Part I}

\subsubsection{SNPs selection}

\section{NLGN3}

The gene size of 26,3 KB allowed distribution of the SNPs at nearly equal distances (5-7 KB) and the detection of possible signals from each part of the gene. Figure 2 shows the position of the SNPs on the gene.

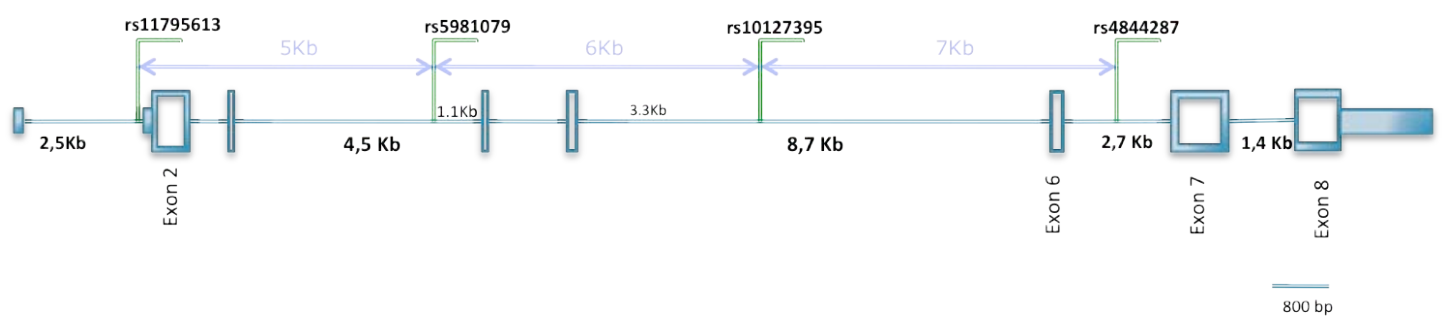

Figure 2. Schematic representation of the NLGN3 gene. Lines represent introns, empty boxes represent exons, filled boxes represent 3'- and 5' UTR regions. The distances between exons are written in black. Distances between SNPs are written in purple.

All SNPs in the NLGN3 gene met the selection criteria for minimal allelic frequency. The minimal allelic frequencies and the SNP alleles are presented in the table below.

$\begin{array}{lll}\text { SNP } & \text { MAF } & \text { Alleles } \\ \text { rs11795613 } & 0.5 & \text { A:G } \\ \text { rs5981079 } & 0.456 & \text { T:C } \\ \text { rs10127395 } & 0.482 & \text { T:G } \\ \text { rs4844287 } & 0.456 & \text { T:C }\end{array}$

Table 1. Single nucleotide polymorphisms (SNPs) in the NLGN3 gene. The minor allele frequency (MAF) for each SNP and the SNP alleles are shown. 


\section{SHANK3}

This gene is $58,57 \mathrm{~KB}$ in size and was covered by nine SNPs, which are shown in the figure below.

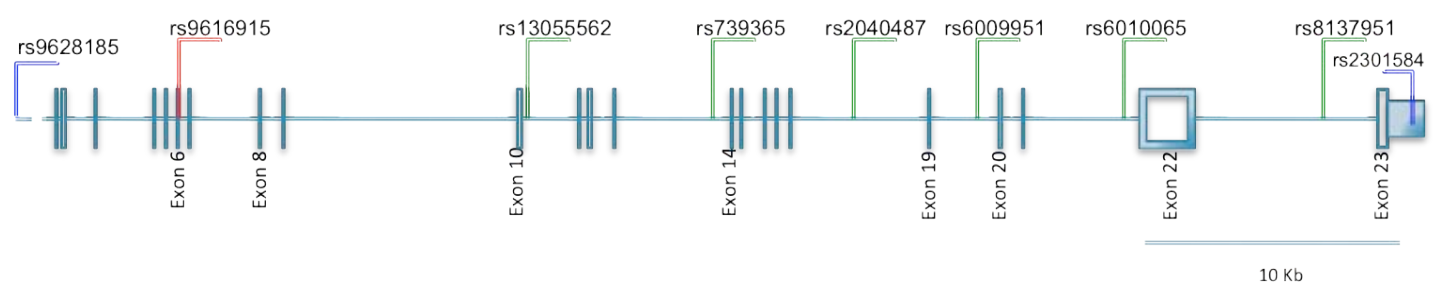

Figure 3: Schematic representation of the SHANK3 gene. Lines represent introns, empty boxes represent exons, filled boxes represent 3' UTR region.

Table 2 shows the characteristics of the SNPs selected for SHANK3. Eight SNPs have minor allele frequencies close to 0.5 . Although rs2301584 has a smaller MAF, its location is in the 3'UTR region, involved in expression regulation (Barrett, et al. 2012; Pichon, et al. 2012). Another SNP, rs9628185, is situated $3 \mathrm{~KB}$ away from the transcription start site, in the promoter region, which in case of significant association signals might shed light directly onto expression regulation mechanisms (Ayoubi and Van De Ven 1996; JuvenGershon and Kadonaga 2010).

$\begin{array}{lll}\text { SNP } & \text { MAF } & \text { Alleles } \\ \text { rs9628185 } & 0.478 & \text { T:C } \\ \text { rs9616915 } & 0.491 & \text { C:T } \\ \text { rs13055562 } & 0.491 & \text { A:G } \\ \text { rs739365 } & 0.321 & \text { C:T } \\ \text { rs2040487 } & 0.429 & \text { A:G } \\ \text { rs6009951 } & 0.402 & \text { C:T } \\ \text { rs6010065 } & 0.441 & \text { G:C } \\ \text { rs8137951 } & 0.286 & \text { G:A } \\ \text { rs2301584 } & 0.164 & \text { G:A }\end{array}$

Table 2 Single nucleotide polymorphisms (SNPs) in the SHANK3 gene. The minor allele frequency (MAF) for each SNP and the SNP alleles are shown 


\section{NLGN4X}

Since the gene size, at $337,8 \mathrm{~KB}$, is relatively big, the SNPs were selected to specifically cover the gene region, where mutations have been reported to cause autism, i.e. in exon 5: K378R reported by (Pampanos, et al. 2009), 1253delAG reported by (Laumonnier, et al. 2004) and 1186insT reported by (Jamain, et al. 2003). The rs2290488 was chosen due to its position in the exonic region of the splice variant NM_020742.3, while the slice variants NM_181332.2 and NM_001282145.1 map to the intronic region (Kent, et al. 2002). The rs 1455316 SNP is 3237 base pairs away from the coding sequence start at the 5 ' end.

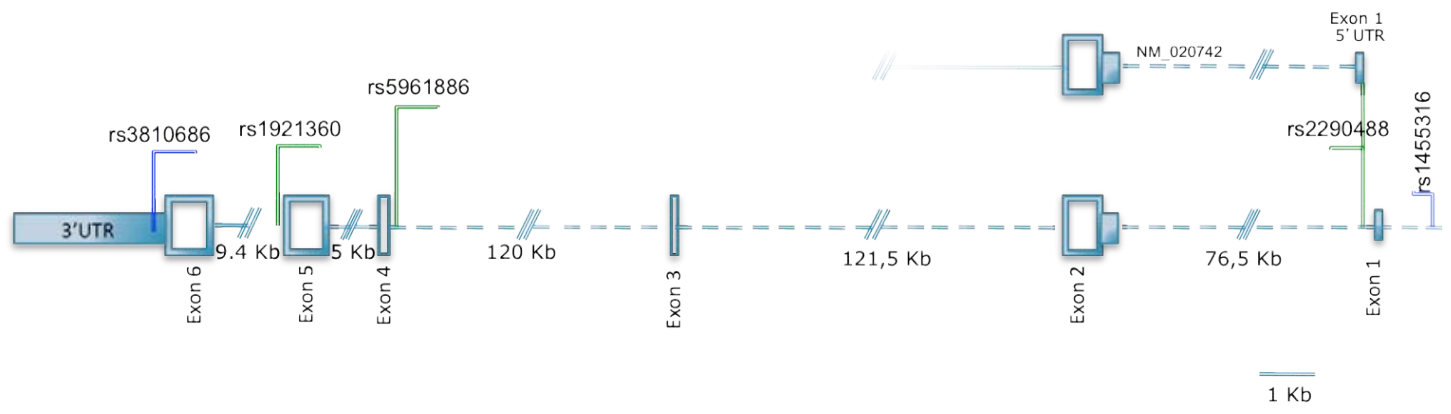

Figure 4 Schematic representation of the NLGN4X gene. Lines represent introns, empty boxes represent exons, filled boxes represent $3^{\prime}$ ' and $5^{\prime}$ ' UTR region

$\begin{array}{lll}\text { SNP } & \text { MAF } & \text { Alleles } \\ \text { rs1455316 } & 0.244 & \text { G:A } \\ \text { rs2290488 } & 0.4 & \text { C:G } \\ \text { rs5961886 } & 0.494 & \text { C:T } \\ \text { rs1921360 } & 0.325 & \text { G:T } \\ \text { rs3810686 } & 0.3 & \text { C:T }\end{array}$

Table 3 Single nucleotide polymorphisms (SNPs) in the NLGN4X gene. The minor allele frequency (MAF) for each SNP and the SNP alleles are shown 


\section{NLGN4Y}

Due to a repressed meiotic recombination in the y-chromosome (Sun and Heitman 2012) only two markers were chosen. Both have relatively low MAF, since they are situated on an infrequently recombining chromosome, but both lie in close vicinity to the exons (Fig $5)$.

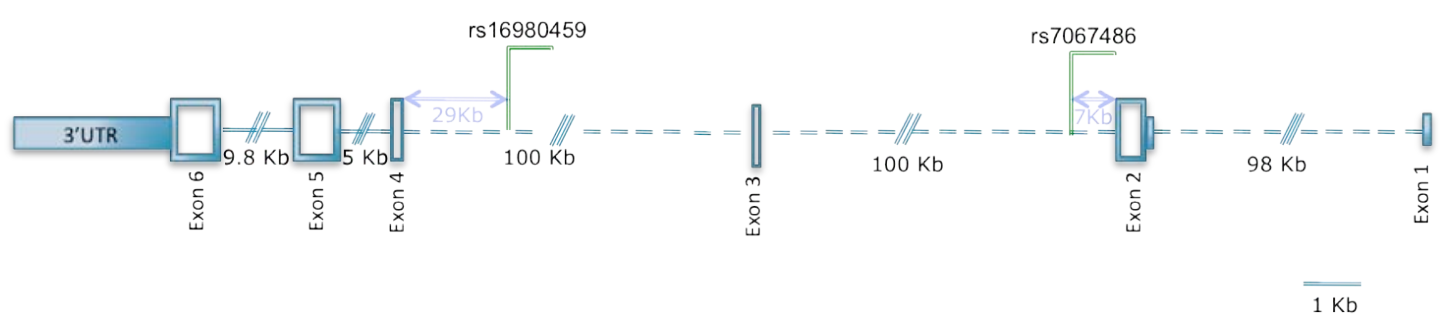

Figure 5 Schematic representation of the NLGN4Y gene. Lines represent introns, empty boxes represent exons, filled boxes represent 3' UTR and 5' UTR regions

$\begin{array}{lll}\text { SNP } & \text { MAF } & \text { Alleles } \\ \text { rs7067486 } & 0.3 & \text { G:A } \\ \text { rs16980459 } & 0.15 & \text { A:G }\end{array}$

Table 4 Single nucleotide polymorphisms (SNPs) in the NLGN4Y gene. The minor allele frequency (MAF) for each SNP and the SNP alleles are shown

\section{NRXN1}

The gene is very big in size, spreading over $1 \mathrm{MB}$ on chromosome 2. The extensive coverage of the gene with SNPs positioned in regular intervals would be difficult to accomplish due to its big size. Thus, two markers were selected and both are situated in the UTR- regions.

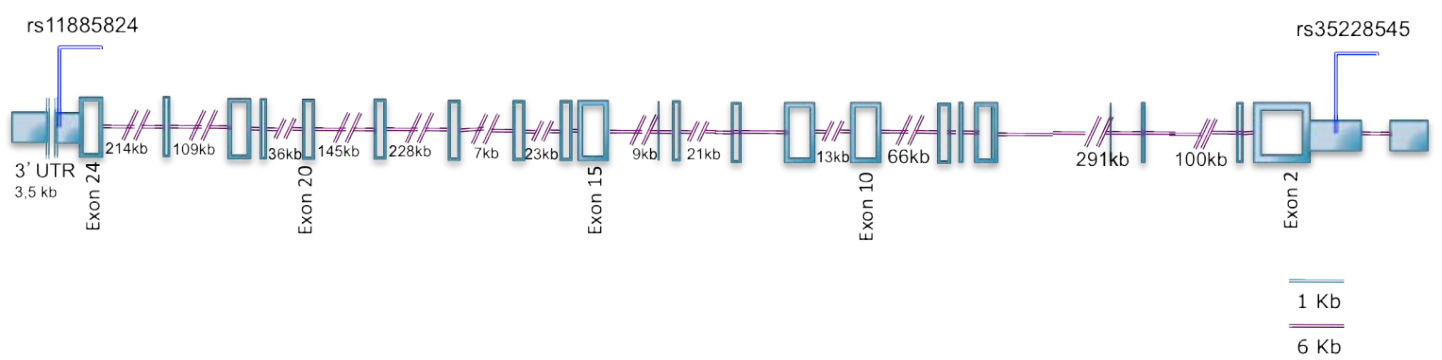

Figure 6 Schematic representation of the NRXN1 gene. Lines represent introns, empty boxes represent exons, filled boxes represent 3' UTR and 5' UTR regions 


$\begin{array}{lll}\text { SNP } & \text { MAF } & \text { Allel } \\ \text { SNP2 } & 0.2 & \text { C:A } \\ \text { SNP1 } & 0.25 & \text { A:G }\end{array}$

Table 5 Single nucleotide polymorphisms (SNPs) in the NRXN1 gene. The minor allele frequency (MAF) for each SNP and the SNP alleles are shown

\subsubsection{Case-control association study}

All SNPs fulfilled the Hardy- Weinberg equilibrium as shown in Table 6.

\begin{tabular}{|l|l|l|}
\hline \multicolumn{2}{|l|}{ NLGN3 (women) } & \multicolumn{2}{l|}{ P controls } \\
\hline rs11795613 & 0,87 & 0,75 \\
\hline rs5981079 & 0,59 & 0,99 \\
\hline rs10127395 & 0,47 & 0,61 \\
\hline rs4844287 & 0,42 & 0,55 \\
\hline SHANK3 & 0,33 & 0,98 \\
\hline rs9628185 & 0,98 & 0,97 \\
\hline rs9616915 & 0,93 & 0,22 \\
\hline rs13055562 & 0,98 & 0,70 \\
\hline rs739365 & 0,25 & 0,99 \\
\hline rs2040487 & 0,66 & 0,84 \\
\hline rs6009951 & 0,81 & 0,99 \\
\hline rs6010065 & 0,20 & 0,99 \\
\hline rs8137951 & \multicolumn{2}{|l|}{} \\
\hline NLGN4X (women) & 0,56 & 0,99 \\
\hline rs1455316 & 0,94 & 0,72 \\
\hline rs2290488 & 0,77 & 0,33 \\
\hline rs5961886 & 0,98 & 0,90 \\
\hline rs1921360 & 0,98 & 0,99 \\
\hline rs3810686 & \multicolumn{2}{|l|}{} \\
\hline
\end{tabular}




\begin{tabular}{|l|l|l|}
\hline \multicolumn{2}{|l|}{ NRXN1 } \\
\hline rs35228545 & 0,65 & 0,48 \\
\hline rs118855824 & 0,79 & 0,49 \\
\hline
\end{tabular}

Table 6 Test for deviation from Hardy-Weinberg-Equilibrium (HWE) for NLGN3 (for women), SHANK3, NLGN4X (for women), and NRXN1.

The case control study did not deliver any significant results. Table 7 lists the results of genomic comparisons for each SNP. For genes in the sex chromosomes, analysis was performed separately for men and women.

\begin{tabular}{|c|c|c|c|c|}
\hline \multicolumn{5}{|c|}{ NLGN4X } \\
\hline & \multicolumn{3}{|c|}{ Genotypes } & $P$-value \\
\hline rs1455316 (MEN) & C & \multicolumn{2}{|r|}{$T$} & \multirow{3}{*}{0.459} \\
\hline Cases $(n=691)$ & $514(74.4 \%)$ & \multicolumn{2}{|c|}{$177(25.6 \%)$} & \\
\hline Controls $(n=657)$ & $477(72.6 \%)$ & \multicolumn{2}{|c|}{$180(25.6 \%)$} & \\
\hline rs2290488 (MEN) & G & \multicolumn{2}{|r|}{ C } & \multirow{3}{*}{0.980} \\
\hline Cases $(n=700)$ & $441(63.0 \%)$ & \multicolumn{2}{|c|}{$259(37.0 \%)$} & \\
\hline Controls $(n=661)$ & $416(62.3 \%)$ & \multicolumn{2}{|c|}{$245(37.1 \%)$} & \\
\hline rs5961886 (MEN) & C & \multicolumn{2}{|r|}{$T$} & \multirow{3}{*}{0.912} \\
\hline Cases $(n=699)$ & $278(39.8 \%)$ & \multicolumn{2}{|c|}{$421(60.2 \%)$} & \\
\hline Controls $(n=660)$ & $265(40.2 \%)$ & \multicolumn{2}{|c|}{$395(59.8 \%)$} & \\
\hline rs1921360 (MEN) & G & \multicolumn{2}{|r|}{$\mathbf{T}$} & \multirow{3}{*}{0.128} \\
\hline Cases $(n=692)$ & $389(56.2 \%)$ & \multicolumn{2}{|c|}{$303(43.8 \%)$} & \\
\hline Controls $(n=660)$ & $398(60.3 \%)$ & \multicolumn{2}{|c|}{$262(39.7 \%)$} & \\
\hline rs3810686 (MEN) & C & \multicolumn{2}{|r|}{$T$} & \multirow{3}{*}{0.209} \\
\hline Cases $(n=700)$ & $401(57.3 \%)$ & \multicolumn{2}{|c|}{$299(42.7 \%)$} & \\
\hline Controls $(n=663)$ & $402(60.6 \%)$ & & $(39.4 \%)$ & \\
\hline rs1455316 (Women) & $\mathrm{CC}$ & TT & TC & \\
\hline Cases $(n=339)$ & $186(54.9 \%)$ & $28(8.3 \%)$ & $125(36.9 \%)$ & 0.306 \\
\hline Controls $(n=461)$ & $228(49.5 \%)$ & $40(8.7 \%)$ & $193(41.9 \%)$ & \\
\hline rs2290488 (Women) & GG & CC & GC & \\
\hline Cases $(n=340)$ & $137(40.3 \%)$ & $43(12.6 \%)$ & $160(47.1 \%)$ & 0.305 \\
\hline Controls $(n=461)$ & $173(37.5 \%)$ & $76(16.5 \%)$ & $212(46.0 \%)$ & \\
\hline
\end{tabular}


Results

\begin{tabular}{|c|c|c|c|c|}
\hline rs5961886 (Women) & CC & TT & TC & \multirow{3}{*}{0.298} \\
\hline Cases $(n=339)$ & $53(15.6 \%)$ & $132(38.9 \%)$ & $154(45.4 \%)$ & \\
\hline Controls $(n=462)$ & $58(12.6 \%)$ & $172(37.2 \%)$ & $232(50.2 \%)$ & \\
\hline rs1921360 (Women) & GG & TT & TG & \multirow{3}{*}{0.866} \\
\hline Cases $(n=334)$ & $108(32.3 \%)$ & $64(19.2 \%)$ & $162(48.5 \%)$ & \\
\hline Controls $(n=461)$ & $157(34.1 \%)$ & $84(18.2 \%)$ & $220(47.7 \%)$ & \\
\hline rs3810686 (Women) & CC & TT & TC & \multirow{3}{*}{0.695} \\
\hline Cases $(n=339)$ & $108(31.9 \%)$ & $66(19.5 \%)$ & $165(48.7 \%)$ & \\
\hline Controls $(n=462)$ & $158(34.2 \%)$ & $81(17.5 \%)$ & $223(48.3 \%)$ & \\
\hline \multicolumn{5}{|c|}{ NLGN3 } \\
\hline & \multicolumn{3}{|c|}{ Genotypes } & $P$-value \\
\hline rs11795613 (MEN) & \multicolumn{2}{|l|}{$\mathbf{A}$} & G & \multirow{3}{*}{0.938} \\
\hline Cases $(n=701)$ & \multicolumn{2}{|c|}{$376(53.6 \%)$} & $5(46.4 \%)$ & \\
\hline Controls $(n=663)$ & \multicolumn{2}{|l|}{$357(53.8 \%)$} & $6(46.2 \%)$ & \\
\hline rs5981079 (MEN) & \multicolumn{2}{|l|}{$\mathbf{T}$} & C & \multirow{3}{*}{0.988} \\
\hline Cases $(n=701)$ & \multicolumn{2}{|l|}{$418(59.6 \%)$} & $3(40.4 \%)$ & \\
\hline Controls $(n=662)$ & \multicolumn{2}{|c|}{$395(59.7 \%)$} & $7(40.3 \%)$ & \\
\hline rs10127395 (MEN) & \multicolumn{2}{|l|}{$\mathbf{T}$} & G & \multirow{3}{*}{0.993} \\
\hline Cases $(n=699)$ & $424(60.7 \%)$ & & $(39.3 \%)$ & \\
\hline Controls $(n=663)$ & $402(60.6 \%)$ & & $(39.4 \%)$ & \\
\hline rs4844287 (MEN) & $T$ & & C & \\
\hline Cases $(n=701)$ & $431(61.5 \%)$ & & $(38.5 \%)$ & 0.774 \\
\hline Controls $(n=662)$ & $402(60.7 \%)$ & & $(39.3 \%)$ & \\
\hline rs11795613 (Women) & AA & GG & GA & \\
\hline Cases $(n=340)$ & $102(30.0 \%)$ & $74(21.8 \%)$ & $164(48.2 \%)$ & 0.672 \\
\hline Controls $(n=461)$ & $128(27.8 \%)$ & $111(24.1 \%)$ & $222(48.2 \%)$ & \\
\hline rs5981079 (Women) & TT & CC & TC & \\
\hline Cases $(n=340)$ & $141(41.5 \%)$ & $50(14.7 \%)$ & $149(43.8 \%)$ & 0.069 \\
\hline Controls $(n=461)$ & $155(33.6 \%)$ & $82(17.8 \%)$ & $224(48.6 \%)$ & \\
\hline rs10127395 (Women) & TT & GG & TG & \\
\hline Cases $(n=340)$ & $145(42.6 \%)$ & $49(14.4 \%)$ & $146(42.9 \%)$ & 0.827 \\
\hline Controls $(n=462)$ & $187(40.5 \%)$ & $69(14.9 \%)$ & $206(44.6 \%)$ & \\
\hline rs4844287 (Women) & TT & CC & TC & 0.907 \\
\hline
\end{tabular}




\begin{tabular}{|c|c|c|c|c|}
\hline Cases $(n=340)$ & 146 (42.9\%) & $49(14.4 \%)$ & $145(42.6 \%)$ & \\
\hline Controls $(n=461)$ & $191(41.4 \%)$ & $67(14.5 \%)$ & $203(44.0 \%)$ & \\
\hline \multicolumn{5}{|c|}{ SHANK3 } \\
\hline & \multicolumn{3}{|c|}{ Genotypes } & P-value \\
\hline rs9628185 & TT & CC & TC & \multirow{3}{*}{0.476} \\
\hline Cases $(n=1040)$ & $281(27.0 \%)$ & $263(25.3 \%)$ & $496(47.7 \%)$ & \\
\hline Controls $(n=1128)$ & $286(25.4 \%)$ & $275(24.4 \%)$ & $567(50.3 \%)$ & \\
\hline rs9616915 & TT & CC & TC & \multirow{3}{*}{0.843} \\
\hline Cases $(n=1039)$ & $236(22.7 \%)$ & $288(27.7 \%)$ & $515(49.6 \%)$ & \\
\hline Controls $(n=1130)$ & $245(21.7 \%)$ & $319(28.2 \%)$ & $566(50.1 \%)$ & \\
\hline rs13055562 & GG & AA & GA & \multirow{3}{*}{0.614} \\
\hline Cases $(n=1006)$ & $198(19.7 \%)$ & $318(31.6 \%)$ & $490(480.7 \%)$ & \\
\hline Controls $(n=1091)$ & $231(21.2 \%)$ & $349(32.0 \%)$ & $511(46.8 \%)$ & \\
\hline rs739365 & TT & CC & TC & \multirow{3}{*}{0.314} \\
\hline Cases $(n=1036)$ & $95(9.2 \%)$ & $507(48.9 \%)$ & $434(41.9 \%)$ & \\
\hline Controls $(n=1128)$ & $123(10.9 \%)$ & $525(46.5 \%)$ & $480(42.6 \%)$ & \\
\hline rs2040487 & GG & AA & GA & \multirow{3}{*}{0.333} \\
\hline Cases $(n=993)$ & $192(19.3 \%)$ & $342(34.4 \%)$ & $459(46.2 \%)$ & \\
\hline Controls $(n=1106)$ & $187(16.9 \%)$ & $385(34.8 \%)$ & $534(48.3 \%)$ & \\
\hline rs6009951 & $\mathrm{CC}$ & TT & CT & \\
\hline Cases $(n=1039)$ & 347 (33.4\%) & $173(16.7 \%)$ & $519(50.0 \%)$ & 0.814 \\
\hline Controls $(n=1030)$ & $392(34.7 \%)$ & $183(16.2 \%)$ & $555(49.1 \%)$ & \\
\hline rs6010065 & GG & CC & CG & \\
\hline Cases $(n=1033)$ & $305(29.5 \%)$ & $206(19.9 \%)$ & $522(50.5 \%)$ & 0.059 \\
\hline Controls $(n=1130)$ & $298(26.4 \%)$ & $269(23.8 \%)$ & $563(49.8 \%)$ & \\
\hline rs8137951 & GG & AA & GA & \\
\hline Cases $(n=1036)$ & $519(50.1 \%)$ & $72(6.9 \%)$ & $445(43.0 \%)$ & 0.096 \\
\hline Controls $(n=1129)$ & $542(48.0 \%)$ & $107(9.5 \%)$ & $480(42.5 \%)$ & \\
\hline & & $\overline{G N 4 Y}$ & & \\
\hline rs7067486 & A & & G & \\
\hline Cases $(n=692)$ & $310(44.8$ & & $(55.2 \%)$ & 0.058 \\
\hline Controls $(n=660)$ & $262(39.7$ & & $3(60.3 \%)$ & \\
\hline rs16980459 & $\bar{A}$ & & $\mathbf{G}$ & \\
\hline Cases $(n=696)$ & $640(92.0$ & & $(8.0 \%)$ & 0.059 \\
\hline Controls $(n=659)$ & $623(94.5$ & & $(5.5 \%)$ & \\
\hline
\end{tabular}


Results

\begin{tabular}{|c|c|c|c|c|}
\hline \multicolumn{5}{|c|}{ NRXN1 } \\
\hline rs35228545 & AA & GG & AG & \multirow{3}{*}{0.323} \\
\hline Cases $(n=1039)$ & $508(48.9 \%)$ & $103(9.9 \%)$ & $428(41.2 \%)$ & \\
\hline Controls $(n=1127)$ & $534(47.4 \%)$ & $97(8.6 \%)$ & $496(44.0 \%)$ & \\
\hline rs118855824 & AA & CC & AC & \multirow{3}{*}{0.118} \\
\hline Cases $(n=1035)$ & $33(3.2 \%)$ & $716(69.2 \%)$ & $286(27.6 \%)$ & \\
\hline Controls $(n=1126)$ & $34(3.0 \%)$ & $735(65.3 \%)$ & $357(31.7 \%)$ & \\
\hline
\end{tabular}

Table 7 Case - control study of the synaptic genes in the GRAS population. P-value reported is derived from the genotypic comparisons. 


\subsubsection{Screen testing of dnrxn and D. melanogaster}

Sound recordings, obtained from Canton $S$ (wild type) flies, demonstrated a normal pattern characteristic of courtship song for Drosophila melanogaster (Tauber and Eberl 2003). Figure 7 demonstrates how a male wild type fly switches repeatedly from sine to pulse song.

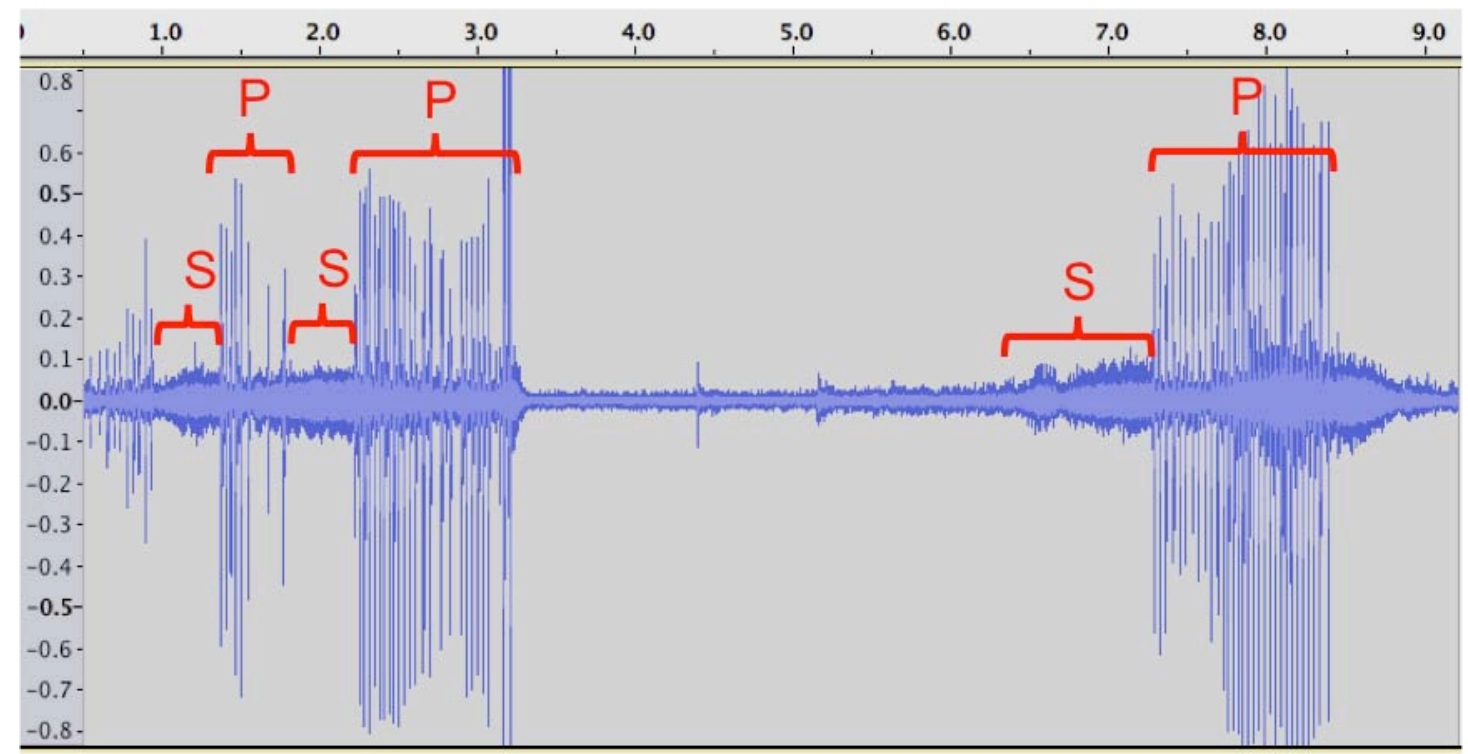

Figure 7 Courtship song of a male Canton S D. Melanogaster. Sine (S) and Pulse (P) songs are highlighted.

Figure 8 represents the pulse song of the Cantos $S$ fly in an enlarged time scale. The average Inter Pulse Interval $(I P I)$ : $I P I=36( \pm 3,8)$ milliseconds (Fig8). This corresponds to the normal average pulse song characteristics of the wild type Drosophila (Tauber and Eberl 2003).

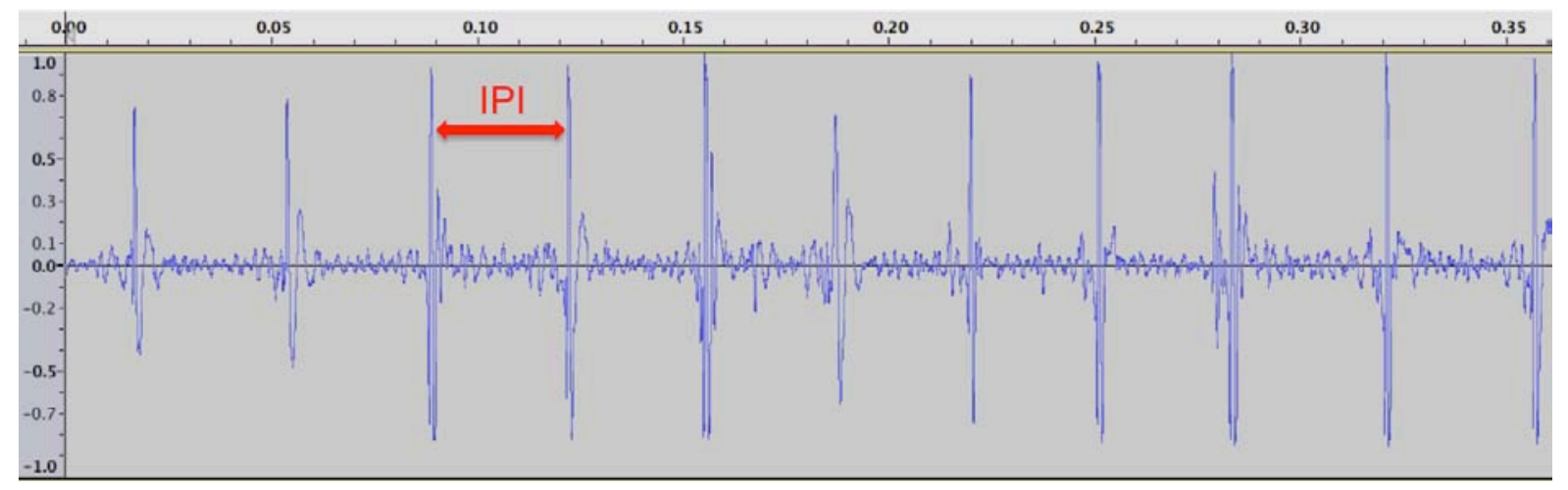

Figure 8 Pulse song of a Canton $\mathbf{S}$ drosophila in an enlarged time scale.

The Interpulse Interval (IPI) is highlighted. 


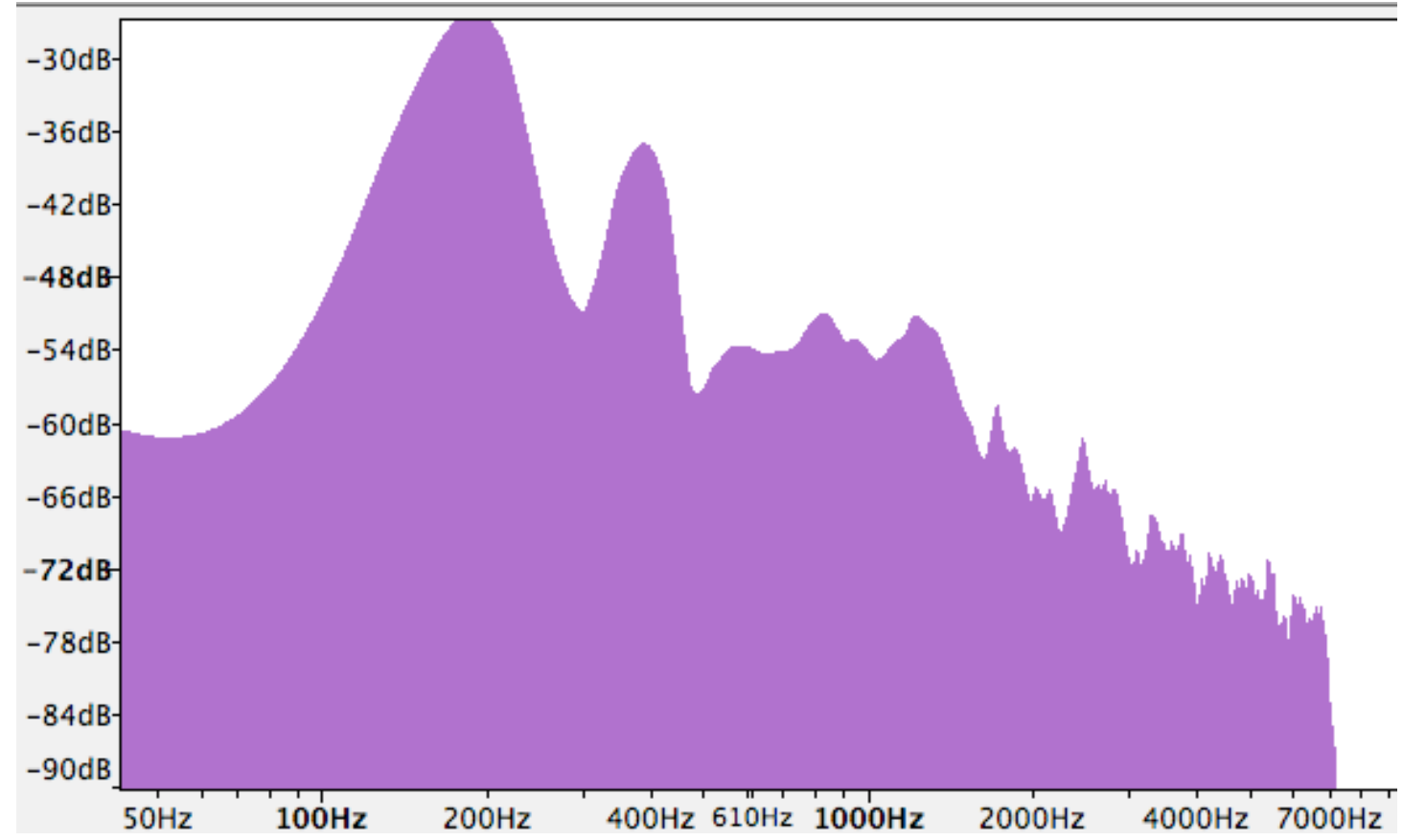

Figure 9 Spectral analysis of the sine song of the Canton $S$ (wild type) male song. The

Peak frequency is $190 \mathrm{~Hz}$.

Twelve trials were run in two sets of experiments. Eight mutant male flies and four male Canton $\mathrm{S}$ (wild type) were tested. Table 8 shows the duration of the sine and pulse songs of the $n r x-1241 / D f E x c e / 6191$ and control flies, measured between the $1^{\text {std }}$ and $6^{\text {th }}$ minutes of the recording period.

\begin{tabular}{|c|c|c|c|}
\hline \multicolumn{3}{|c|}{ Set 1 } & Pulse (sec) \\
\hline Muleject & Genotype & Sine (sec) & 4,5 \\
\hline Male2 & Mutant & silent & silent \\
\hline Male3 & Mutant & 6,5 & 2,4 \\
\hline Male4 & Mutant & silent & silent \\
\hline Male5 & CS & silent & silent \\
\hline \multicolumn{4}{|c|}{ Set 2 } \\
\hline Male1 & Mutant & $<1$ & single pulses \\
\hline Male2 & Mutant & 2 & single pulses \\
\hline Male3 & CS & 60 & 53 \\
\hline Male4 & Mutant & 2 & single pulses \\
\hline Male5 & Mutant & 1 & single pulses \\
\hline Male6 & CS & 43 & 38 \\
\hline Male7 & Mutant & 1 & 6,3 \\
\hline
\end{tabular}

Table 8 Courtship songs of male mutant nrx-1241/DfExce/6191 and control flies. The duration of the sine and pulse songs (where applicable) is shown. 
Two of the eight flies did not produce any sound. The video recording has showed that they tried to initiate the courtship song production, but stayed with one wing extended and could not produce any sound, which is indicative of a severe locomotor impairment (Fig 9). The other six male mutant $n r x-$ 1241/DfExce/6191 flies produced sounds, however they were abnormal courtship songs. Thus, no pulse song was produced, which normally should contain from 2 to 50 pulses per train (Tauber and Eberl 2003), but single pulses were observed instead (Fig 10). The sine song produced by male mutant nrx-1241/DfExcel6191 flies was also abnormal. The overall length of the sine song of the mutant flies was shorter than that of wild type flies.

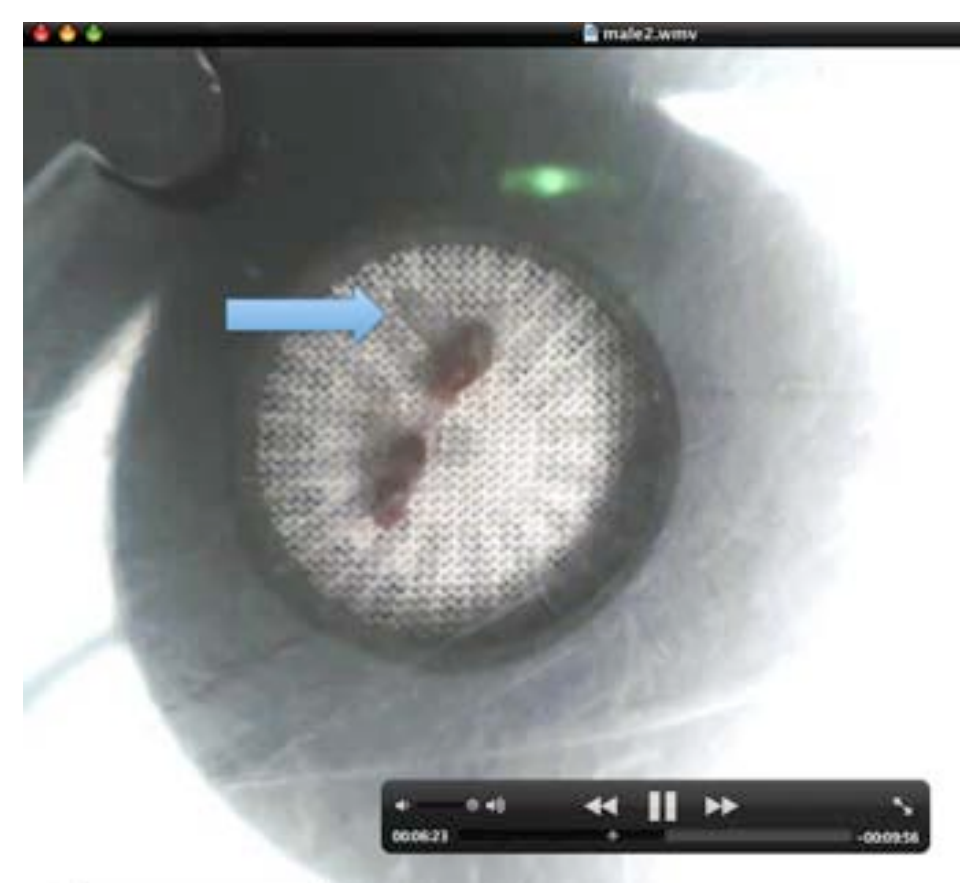

\section{$06: 23: 53$}

Figure 10: Male mutant $n r x-1241 / D f E x c e / 6191$ tries to initiate the courtship song production, but the wing stays extended, indicating a locomotor impairment. 


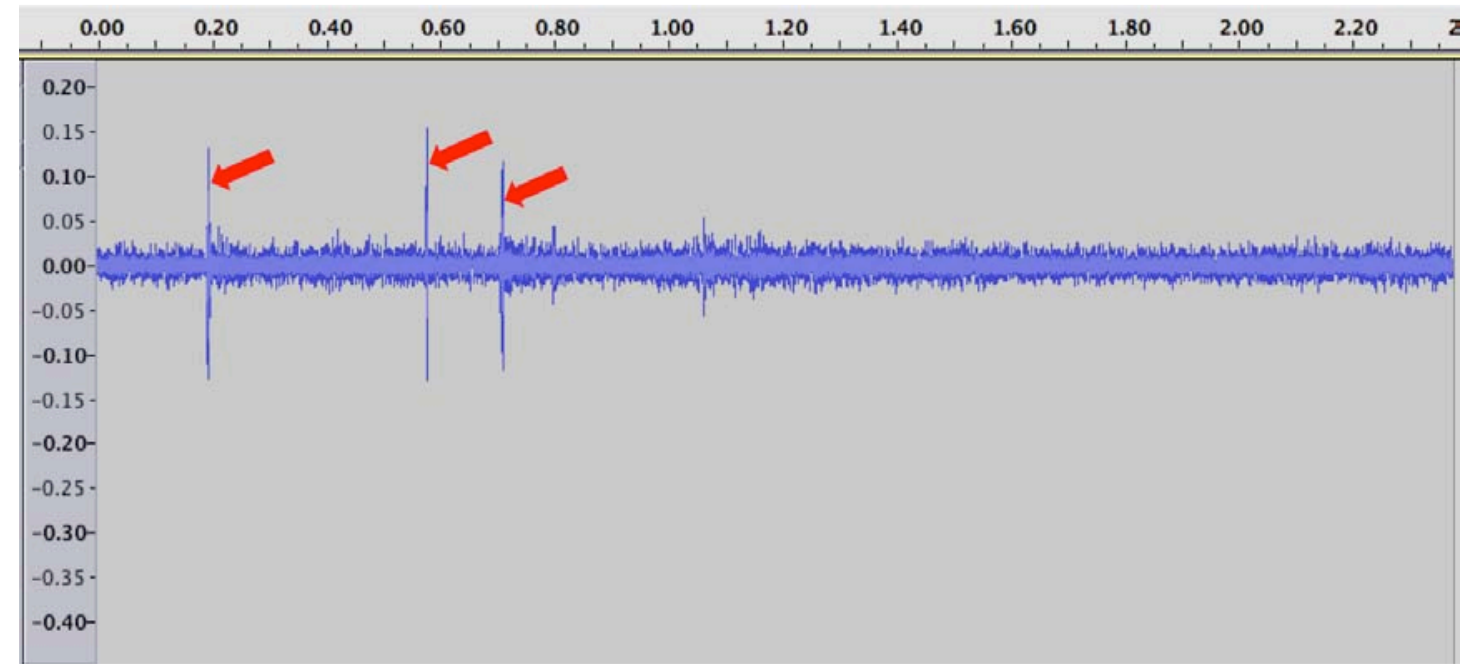

Figure 11: Male mutant $n r x-1241 / D f E x c e / 6191$ is only able to produce single pulses not accompanied by a sine song.

The sine song produced my $n r x-1241 / D f E x c e / 6191$ flies also arrived in short bouts of about 0,1 seconds (Fig 12) and had abnormal spectral characteristics (max frequencies $646 \mathrm{~Hz}$ and $1175 \mathrm{~Hz}$ ) as compared to the Canton $\mathrm{S}$ flies (max frequency $190 \mathrm{~Hz}$ ) (Fig 9 and Fig13).

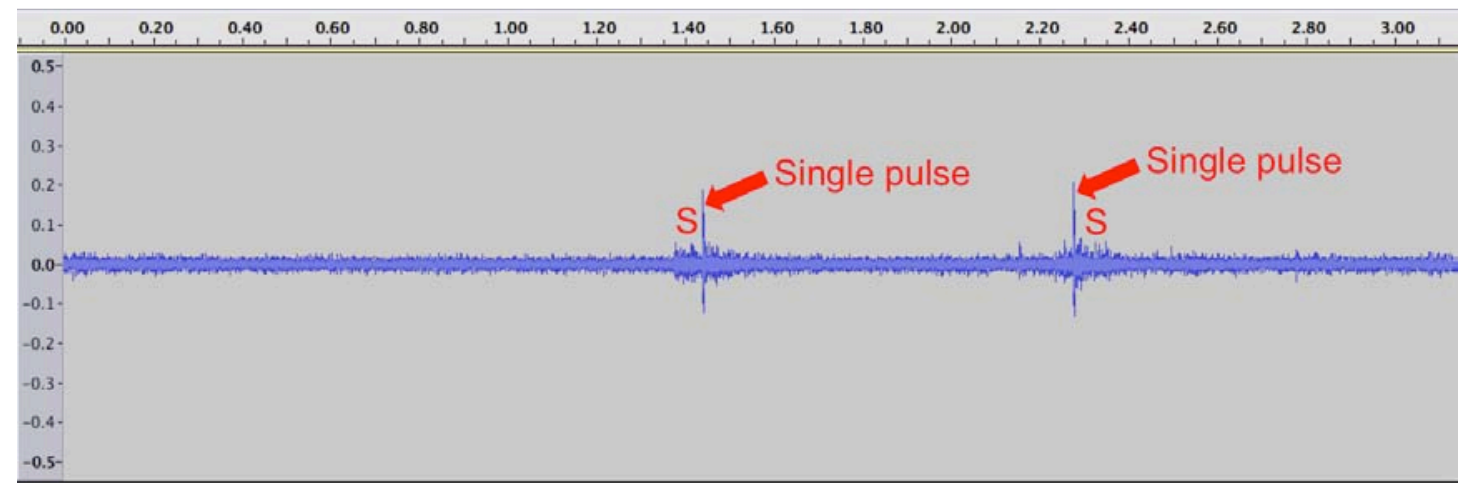

Figure 12: Abnormal sine song (S) of $n r x-1241 / D f E x c e / 6191$ male fly. The song is produced in very short bouts, with reduced amplitude and accompanied by single pulses. 


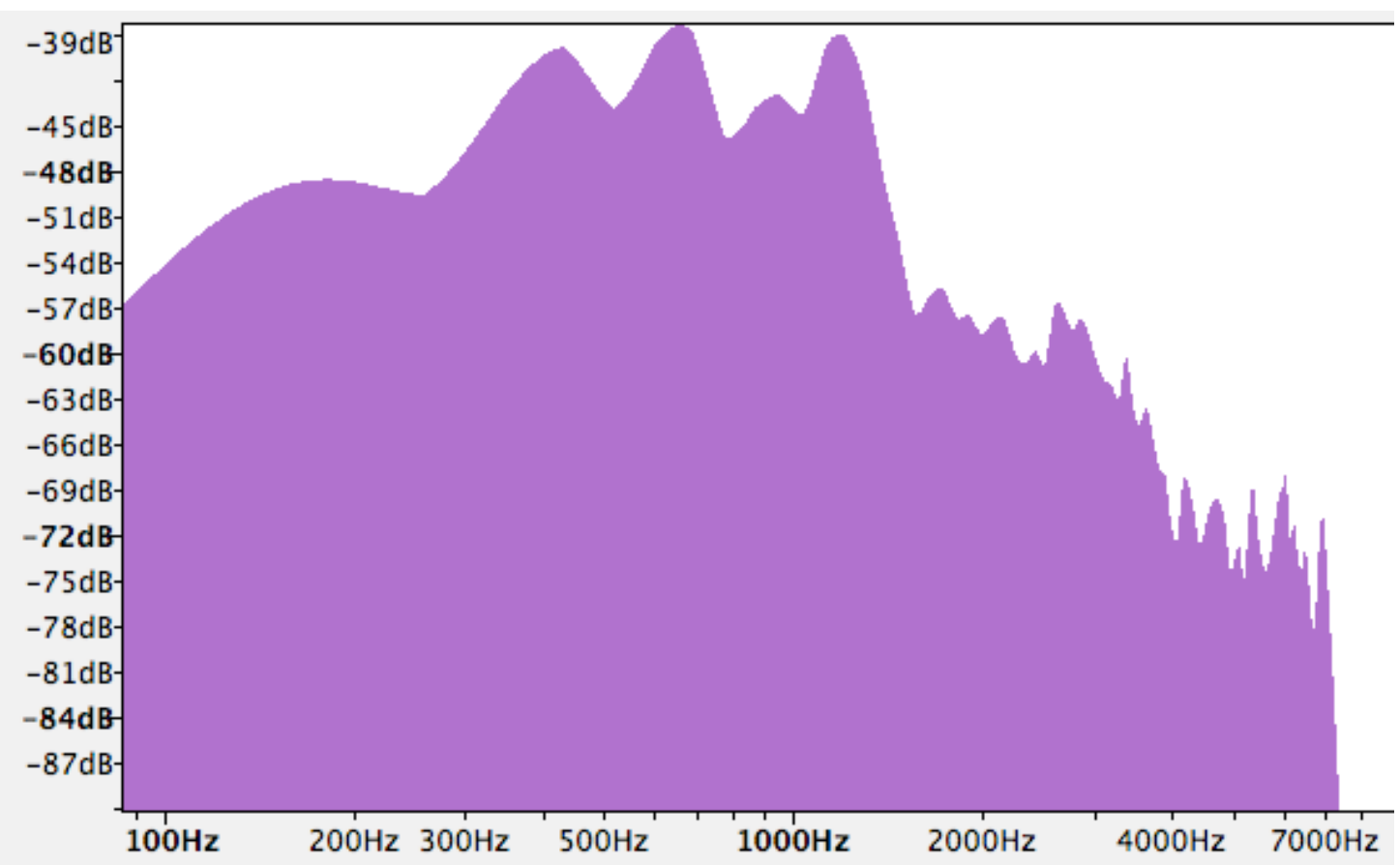

Figure 13: Spectrum analysis of the sine song of the $n r x-1241 / D f E x c e / 6191$ male fly shows abnormal characteristics of song; peak frequencies at $646 \mathrm{~Hz}$ and $1175 \mathrm{~Hz}$.

Additionally, the screen testing of $n r x-1241 / D f E x c e / 6191$ mutant male flies showed that the flies are either silent or produce a severely abnormal pattern of the courtship song, which is due to their severe locomotor impairment.

\subsubsection{Behavioral assay of the dnl2 mutant flies}

The detailed description of the results is provided in the Appendix in Hahn et al. 2013. Here the results summary is provided.

The acoustic signals of the dn/2-deficient flies had overall characteristics similar to those of the wild type flies, though the IPI of the pulse song was shorter and the volume of the sine song was reduced in $d n l^{K O 17}$ flies compared to the wild type flies.

The median distance of individual flies to all other flies in the arena during the group behavior assay was significantly larger in $d n l^{K O 17}$ mutants than in wild type flies.

In the assay, where two males of equal genotype were assessed during their interaction with a decapitated mature virgin wild type female, the $d n l^{K O 17}$ mutants showed no difference in courtship behavior as compared to wild types. However in the mixed assay, where one $d n l^{K 017}$ mutant was competing 
with one wild type male, the mutant flies performed significantly less courtship, initiated less courtship attempts, and the courtship bouts were shorter than in the wild type males. Moreover, a large proportion of mutant males did not initiate courtship at all while competing with the wild type males in the mixed assay. The durations of agonistic interaction in the equal genotype and mixed assays was reduced in the $d n l^{K O 17}$ mutants as compared to the wild types. The results are suggestive of a reduced motivation in the males. Furthermore, no difference was found in the general activity and peripheral sensory processing of the mutant or the wild type flies.

\subsection{Results Part II}

\subsubsection{Demographic and clinical characteristics of four rapid cyclers}

Four rapid cycling patients, one female (born in 1958) and three male (born in 1961, 1941 and 1959) were included in the study. The detailed sociodemographic and clinical data is presented in Table 1 in the attached manuscript (Gurvich et al, in press), in the appendix.

\subsubsection{Bipolar and monopolar patients cohort description}

43 patients with bipolar affective disorder (male/female=24/19; mean age $50.0 \pm 12.1$ years; mean age at disease onset: $34.5 \pm 11.6$ years; mean number

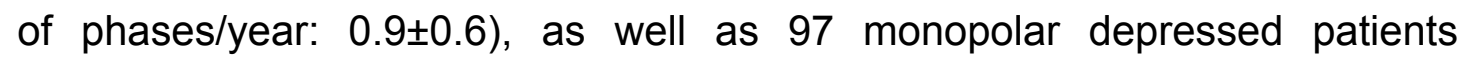
(male/female=42/55; mean age 49.4 \pm 15.0 years; mean age at disease onset: 41.0 \pm 15.7 years; mean number of phases/year: $1.2 \pm 1.3$ ) were recruited.

\subsubsection{PTGDS, ARK1C3 and PTGS2/COX2 ${ }_{P A N}$ episode specific} expression in rapid cyclers, patients with bipolar disorder and monopolar depression.

Detailed results are provided in the manuscript in the Appendix (Gurvich et al, in press), but here is a short summary.

The expression levels of genes that were previously shown to be differentially regulated in a phase specific manner (PTGDS and $A K R 1 C 3)$ were explored 
once more in four patients with rapid cycling bipolar disorder. Furthermore, as a new readout of the prostaglandin synthesis pathway, a ratio of PTS2/COX2 $2_{P A N}$ was determined (the ratio was calculated to specifically address the brain expressed splice variant). P2RX7 (P2X purinoceptor7) was also assessed as a non-prostaglandin related control gene. In order to apply an integrative approach and to assess the expression levels in all four rapid cycling patients, the mean of all measured values per gene regardless of episode was calculated and set to $100 \%$; all individual data obtained were then expressed in \% of this mean value for each gene. Thus, replicating previous findings from the original case report (Begemann, et al. 2008), the levels of PTGDS were significantly higher in the depressed phases of the rapid cycling patients. In contrast, $A K R 1 C 3$ neither showed any significant differential regulation as assessed by integrative approach, nor did it have a consistent trend for such regulation in the individual patient panels. The new PTS2/COX2PAN demonstrated a trend to be increased in depressed episodes in three out of four patients, resulting in borderline significance upon integration. The control gene, $P 2 R X 7$, did not show any steady trend in these 4 patients.

The expression levels of PTGDS, AKR1C3, $P 2 R X 7$ and the ratio PTS2/COX2PAN measured in PBMCs of 43 patients with non-rapid cycling bipolar disorder and 97 monopolar depressed patients did not show any episode specific changes. 


\section{Discussion}

\subsection{Part I}

\subsubsection{Synaptic genes and schizophrenia risk}

A major involvement of synaptic function and homeostasis in autism spectrum disorders is well documented (Bourgeron 2009). This study aimed to further explore the association of several synaptic genes, NLGN3, NLGN4X, NLGN4Y, SHANK3 and NRXN1, previously shown to play an important role in autism spectrum disorders (Durand, et al. 2007; Jamain, et al. 2003; Szatmari, et al. 2007) with neuropsychiatric risk. Altered synaptic functioning has also been reported to be pivotal in another psychiatric disorder schizophrenia (Yin, et al. 2012) - which has a phenotypic and genetic overlap with autism spectrum disorders (Owen, et al. 2011). Thus it was intriguing to explore whether the common variation of these synaptic genes plays a role in schizophrenia risk. Therefore, a population of more than one thousand schizophrenic patients, included in the GRAS sample (Ribbe, et al. 2010), was genotyped with respect to the Single Nucleotide Polymorphisms (SNPs) selected to representatively cover each of the five synaptic genes. Then a case-control study was undertaken. The analysis for the sex chromosomelinked genes, NLGN3, NLGN4X and NLGN4Y, was performed separately for men and women. The case-control study did not reveal any association of NLGN3, NLGN4X, NLGN4Y, SHANK3 or NRXN1 with schizophrenia in the GRAS population.

The role of NLGN3, NLGN4X, NLGN4Y and SHANK3 in the etiology of autism was initially described based on mutations leading to major disturbance in gene function, whereas subsequent genome-wide association studies, taking into account common polymorphisms, failed to find any association of these genes with an autistic phenotype (Anney, et al. 2012; Wang, et al. 2009; Weiss, et al. 2009). Thus, it is possible that the lack of association of these genes with schizophrenia is due the fact that those genes only lead to the disease when severely mutated, whereas common polymorphisms do not play a role in the neuropsychiatric disease risk. NRNX1 was associated with 
neuropsychiatric risk based on Copy Number Variants (CNVs), which are far rarer than SNPs. Similarly, the lack of association of NRXN1 with schizophrenia risk in this study may be indicative of the primarily important role of CNVs in the disease risk and not SNPs. However a much more likely explanation is the heterogeneity of the disease group called "schizophrenia".

Whether common polymorphisms (SNPs) in these synaptic genes are important for the subtle phenotype alterations and/or disease symptomatology is a matter of further investigation.

\subsubsection{D. melanogaster as a model of autism}

Previous studies addressed the possible behavioral implications upon knock out of neuroligin and neurexin genes in mice. Specifically, the Nlgn-3 knock out (KO) mice showed a deficit in social novelty and olfaction (Radyushkin, et al. 2009), while the Nlgn4-KO mice showed a deficit in social behavior (Jamain, et al. 2008), and both null mutations had reduced ultrasound vocalization and brain volume. Neuroligins and neurexins are evolutionary conserved (Knight, et al. 2011) and previous studies described $D$. melanogaster mutants lacking genes, which are homologous to human neurexins and neuroligins (Sun, et al. 2011; Zeng, et al. 2007). However, none of the previous studies explored whether flies lacking neurexin and/or neuroligin demonstrate autism related features in behavior.

Thus, $n r x-1241 / D f E x c e / 6191$ mutant $D$. melanogaster, lacking the neurexin 1 gene, and $d n l^{K O 17}$ mutant flies, lacking the neuroligin - 2 gene, were obtained and involved in behavioral testing. Since one of the key features of an autistic phenotype is impaired social interaction, this behavioral read-out was directly addressed, testing the courtship, agonistic and group behavior of the flies. The courtship behavior in male flies includes several steps, one of which is producing a courtship song, consisting of so-called sine and pulse songs; this is performed by a unilateral wing extension and its vibration (Tauber and Eberl 2003). The courtship song of $n r x-1241 / D f E x c e l 6191$ mutant male flies was recorded during their interaction with a female virgin. Two out of eight tested mutants did not produce any sound at all, though the video recording showed that they attempted to initiate courtship song production, but stayed with one wing extended, and were therefore unable produce any 
sound. The other six male mutant nrx-1241/DfExcel6191 flies produced abnormal courtship songs. While a normal courtship song should contain from 2 to 50 pulses per train, a song of $n r x-1241 /$ DfExcel6191 contained only separate pulses. The overall length of their sine song was shorter than the one of the wild type flies. The sine song of nrx-1241/DfExcel6191 flies also came in short bouts of about 0,1 seconds and had abnormal spectral characteristics (max frequencies $646 \mathrm{~Hz}$ and $1175 \mathrm{~Hz}$ ) compared to the Canton $S$ flies (max frequency $190 \mathrm{~Hz}$ ). The fundamental frequency of the sine song of Canton $S$ wild type flies obtained in this study is consistent with previous reports on spectral characteristics of the wild type sine songs (von Schilcher 1976; Wheeler, et al. 1988). These results are indicative of a severe locomotor impairment in the dnrxn1- deficient flies, which did not allow further testing or drawing any conclusions on social interaction of the mutants.

In contrast, dnln2-deficient flies did not show any major locomotor disabilities. The courtship songs of $d n \ln 2$ - mutants showed reduced intensity of the sine song and shorter interpulse intervals of the pulse song. Since dnln2 - mutant flies were shown to have reduced synaptic transmission in the neuromuscular junction (Banovic, et al. 2010; Sun, et al. 2011), reduced amplitude of wing vibration provoked by decreased muscle activation in the NMJ leads to a lower intensity of the sine song. Altered synaptic properties in thoracic circuits, which generate the pulse song pattern, and/or differences in the intensity of their activation by descending brain neurons is likely responsible for the reduced interpulse intervals of the pulse song (Clyne and Miesenbock 2008). The courtship song of male $D$. melanogaster aims to make the female more receptive and reduce her activity so that further courtship acts can be performed by the male, finally leading to copulation (Greenspan and Ferveur 2000). Thus, altered acoustic communication in the dnln2 -KO flies, which goes along with reduced ultrasound vocalization in N/gn-3 and N/gn-4 deficient mice (Jamain, et al. 2008; Radyushkin, et al. 2009), lack of locomotor and sensory impairment, and larger interindividual distances in the group behavior assay are clearly indicative of an impaired social interaction between these mutant flies. This, in turn, may be due to compromised information processing in the central nervous system circuits responsible for behavior initiation and coordination. Mushroom bodies are the structures 
which play a role in these processes in insects (Popov, et al. 2005). Interestingly, the neuroligin and neurexin expression in mushroom bodies of a honeybee was shown to be regulated by social exposure (Biswas, et al. 2010). This further supports the role of neuroligin- neurexin complexes in social behavior across species and provides intriguing perspectives for studying neuroligin - deficient Drosophila.

\subsection{Part II: Prostaglandin related genes in rapid cycling bipolar disorder}

This study was initiated based on a previous report of a female case with extreme rapid cycling, where genes involved in prostaglandin metabolism were shown to be regulated in a phase-specific manner (Begemann, et al. 2008). For further investigation of the prostaglandin related genes in rapid cycling syndrome, four patients suffering from this disease were included in the study and expression levels of PTGDS and AKR1C3 were measured in the PBMCs from various phases. Indeed, the phase specific expression pattern of PTGDS was replicated in this study, with significantly more expression of the gene during depression. In contrast, AKR1C3 was not differentially regulated in depressive or in manic phases in this study. AKR1C3 and PTGDS are closely related functionally. The product of PTGDS, Prostaglandin D2, has an important role in sleep regulation (Matsumura, et al. 1994; Urade and Hayaishi 2011), while sleep pattern is known to be disturbed in bipolar disorder (Plante and Winkelman 2008). A strong stimulation or accumulation of $\mathrm{PGD}_{2}$ over the course of severe rapid cycling syndrome may lead to a more pronounced induction of AKR1C3 (Mantel, et al. 2012), which could explain the upregulation of $A K R 1 C 3$ in the initial report of extreme symptom severity (Begemann, et al. 2008) and not in the present study, where the patients' psychopathology was less severe.

Furthermore, a new read-out, PTGS2/COX2 $2_{P A N}$, specifically addressing the expression levels of a brain expressed cyclooxygenase isoform, showed a differential phase dependent expression pattern in pooled PBMC samples during depressive episodes. This further supports a strong involvement of the prostaglandin cascade regulation in the course of rapid cycling syndrome. 
No significant results, however, were obtained when expression of these genes was estimated in a phase specific manner in patients with non-rapid cycling bipolar disorder and monopolar depression. It is also important to mention that only a limited number of samples were available from these patients after a 3-year recruitment period, due to rare episode occurrence in non-rapid cycling individuals. This did not allow application of the integrative approach while analyzing the data. Thus these negative findings in non-rapid cycling bipolar patients and monopolar depressed patients may be a result of inter-individual mean expression variability. 


\section{Outlook}

The case-control study did not reveal significant association between common genetic variants in autism related synaptic genes and schizophrenia risk. As discussed previously, this may be due to the fact that only severe gene disruptions may lead to disease, as shown by case reports on autism. Common variation in these genes however may contribute to different aspects of symptomatology of the neuropsychiatric disorders, such as autistic features in schizophrenia. This can be studied by careful analysis of autism related phenotypic readouts and their association with common genetic variants of the synaptic genes, using the PGAS approach (Begemann, et al. 2010). Furthermore, patients having the genotype associated with a more pronounced autistic symptomatology may be selected as donors of fibroblasts, which can be reprogrammed to neurons using an induced pluripotent stem cell technique. This will allow for the application of electrophysiological tests on neurons directly obtained from patients with important genotype-phenotype correlations. Indeed this ambitious and promising project is currently running in our lab on the basis of the GRAS sample.

The present study revealed that dnlgn2 -KO Drosophila melanogaster demonstrate impaired social interaction, as measured by courtship, agonistic and group behavior. It would be intriguing to perform rescue experiments to further validate present findings. Also it would important to assess if other Drosophila neuroligins, namely dnlgn 3 and $d n \operatorname{lgn} 4$ ( $d n \ln 1$ is not expressed in the nervous system), work as counterparts of dnlgn2, allowing formation of proper excitation/inhibition balance, which may be disturbed in autism (Rubenstein and Merzenich 2003). Additionally, based on findings in honeybees that indicate a regulatory role of social exposure in the expression of neuroligins and neurexins (Biswas, et al. 2010), it would interesting to investigate if similar effects may be seen in Drosophila. 
This study also provided further evidence that genes involved in the prostaglandin synthesis cascade are differentially regulated across phases of rapid cycling syndrome. This provides ground for a prospective study in a larger sample of rapid cycling patients and implicates medical intervention strategies, e.g. with cyclooxygenase inhibitors, that would potentially revalidate the compassionate use of medication, as suggested in the initial case report (Begemann, et al. 2008). This would contribute to the development of novel treatment approaches for rapid cycling bipolar disorder, which is hardly manageable with the existing conventional treatment approaches. It would also be intriguing to further explore the gene expression patterns in patients with non-rapid cycling bipolar disorder and monopolar depression in a larger sample, including individuals for a longitudinal followup, importantly ensuring repetitive blood sample collection in several phases for each patient. This will allow for better controlling of inter-individual variability upon data analysis, which may mask existing phase dependent gene expression patterns. Finally, no construct valid animal model is available for bipolar disorder. This study provides evidence, which may lead to the creation of a transgenic mouse model with inducible and reversible PTGDS expression in the brain, thus allowing the study of behavioral consequences of episode specific gene expression changes. 


\section{References}

Abrahams, B. S., and D. H. Geschwind

2008 Advances in autism genetics: on the threshold of a new neurobiology. Nat Rev Genet 9(5):341-55.

Akhondzadeh, S., et al.

2009 Clinical trial of adjunctive celecoxib treatment in patients with major depression: a double blind and placebo controlled trial. Depress Anxiety 26(7):607-11.

Alarcon, M., et al.

2008 Linkage, association, and gene-expression analyses identify CNTNAP2 as an autism-susceptibility gene. Am J Hum Genet 82(1):150-9.

AmericanPsychiatricAssociation

2013 Diagnostic and statistical manual of mental disorders fifth edition. Washington DC, Longon, England.

Anney, R., et al.

2012 Individual common variants exert weak effects on the risk for autism spectrum disorderspi. Hum Mol Genet 21(21):4781-92.

Arons, M. H., et al.

2012 Autism-associated mutations in ProSAP2/Shank3 impair synaptic transmission and neurexin-neuroligin-mediated transsynaptic signaling. The Journal of neuroscience : the official journal of the Society for Neuroscience 32(43):14966-78.

Auranen, M., et al.

2002 A genomewide screen for autism-spectrum disorders: evidence for a major susceptibility locus on chromosome 3q25-27. American journal of human genetics 71(4):777-90.

Ayoubi, T. A., and W. J. Van De Ven

1996 Regulation of gene expression by alternative promoters. FASEB J 10(4):453-60.

Bagner, D. M., M. R. Melinder, and D. M. Barch

2003 Language comprehension and working memory language comprehension and working memory deficits in patients with schizophrenia. Schizophr Res 60(2-3):299-309.

Bailey, A., et al.

1995 Autism as a strongly genetic disorder: evidence from a British twin study. Psychol Med 25(1):63-77.

Banerjee, S., and M. A. Bhat

2007 Neuron-glial interactions in blood-brain barrier formation. Annu Rev Neurosci 30:235-58.

Banovic, D., et al.

2010 Drosophila neuroligin 1 promotes growth and postsynaptic differentiation at glutamatergic neuromuscular junctions. Neuron 66(5):724-38.

Barrett, L. W., S. Fletcher, and S. D. Wilton

2012 Regulation of eukaryotic gene expression by the untranslated gene regions and other non-coding elements. Cell Mol Life Sci 69(21):3613-34.

Bartak, L., and M. Rutter 
1976 Differences between mentally retarded and normally intelligent autistic children. J Autism Child Schizophr 6(2):109-20.

Baudouin, S. J.

2014 Heterogeneity and convergence: the synaptic pathophysiology of autism. Eur J Neurosci 39(7):1107-13.

Beck, A. T., et al.

1961 An inventory for measuring depression. Archives of general psychiatry 4:561-71.

Begemann, M., et al.

2010 Modification of cognitive performance in schizophrenia by complexin 2 gene polymorphisms. Archives of general psychiatry 67(9):879-88.

Begemann, M., et al.

2008 Episode-specific differential gene expression of peripheral blood mononuclear cells in rapid cycling supports novel treatment approaches. Mol Med 14(9-10):546-52.

Bellack, A. S., et al.

1990 An analysis of social competence in schizophrenia. The British journal of psychiatry : the journal of mental science 156:809-18.

Biswas, S., et al.

2010 Sensory regulation of neuroligins and neurexin I in the honeybee brain. PLoS One 5(2):e9133.

Boomsma, A., et al.

2008 A new symptom model for autism cross-validated in an independent sample. J Child Psychol Psychiatry 49(8):809-16.

Bourgeron, $\mathrm{T}$.

2009 A synaptic trek to autism. Curr Opin Neurobiol 19(2):231-4.

Budreck, E. C., and P. Scheiffele

2007 Neuroligin-3 is a neuronal adhesion protein at GABAergic and glutamatergic synapses. Eur J Neurosci 26(7):1738-48.

Castelloe, P., and G. Dawson 1993 Subclassification of children with autism and pervasive developmental disorder: a questionnaire based on Wing's subgrouping scheme. J Autism Dev Disord 23(2):229-41.

Censarek, P., et al.

2004 Cyclooxygenase COX-2a, a novel COX-2 mRNA variant, in platelets from patients after coronary artery bypass grafting. Thromb Haemost 92(5):925-8.

Chubykin, A. A., et al.

2007 Activity-dependent validation of excitatory versus inhibitory synapses by neuroligin-1 versus neuroligin-2. Neuron 54(6):919-31.

Clyne, J. D., and G. Miesenbock

2008 Sex-specific control and tuning of the pattern generator for courtship song in Drosophila. Cell 133(2):354-63.

Collins, C. A., and A. DiAntonio

2007 Synaptic development: insights from Drosophila. Curr Opin Neurobiol 17(1):35-42.

Condray, R., et al. 
2002 The language system in schizophrenia: effects of capacity and linguistic structure. Schizophrenia bulletin 28(3):475-90.

Constantino, J. N.

2011 The quantitative nature of autistic social impairment. Pediatr Res 69(5 Pt 2):55R-62R.

Constantino, J. N., and R. D. Todd

2003 Autistic traits in the general population: a twin study. Archives of general psychiatry 60(5):524-30.

2005 Intergenerational transmission of subthreshold autistic traits in the general population. Biological psychiatry 57(6):655-60.

Craig, J. S., et al.

2004 Persecutory beliefs, attributions and theory of mind: comparison of patients with paranoid delusions, Asperger's syndrome and healthy controls. Schizophr Res 69(1):29-33.

Dave, M., and A. R. Amin

2013 Yin-Yang regulation of prostaglandins and nitric oxide by PGD2 in human arthritis: reversal by celecoxib. Immunol Lett 152(1):47-54.

De Giacomo, A., and E. Fombonne

1998 Parental recognition of developmental abnormalities in autism. Eur Child Adolesc Psychiatry 7(3):131-6.

DeLisi, L. E.

2001 Speech disorder in schizophrenia: review of the literature and exploration of its relation to the uniquely human capacity for language. Schizophrenia bulletin 27(3):481-96.

Desmond, J. C., et al.

2003 The aldo-keto reductase AKR1C3 is a novel suppressor of cell differentiation that provides a plausible target for the noncyclooxygenase-dependent antineoplastic actions of nonsteroidal antiinflammatory drugs. Cancer Res 63(2):505-12.

DeWitt, D. L., and W. L. Smith

1988 Primary structure of prostaglandin G/H synthase from sheep vesicular gland determined from the complementary DNA sequence. Proc Natl Acad Sci U S A 85(5):1412-6.

Dunner, D. L., and R. R. Fieve

1974 Clinical factors in lithium carbonate prophylaxis failure. Archives of general psychiatry 30(2):229-33.

Durand, C. M., et al.

2007 Mutations in the gene encoding the synaptic scaffolding protein SHANK3 are associated with autism spectrum disorders. Nat Genet 39(1):25-7.

Eastwood, S. L., D. Cotter, and P. J. Harrison

2001 Cerebellar synaptic protein expression in schizophrenia. Neuroscience 105(1):219-29.

Edwards, J., H. J. Jackson, and P. E. Pattison

2002 Emotion recognition via facial expression and affective prosody in schizophrenia: a methodological review. Clin Psychol Rev 22(6):789-832.

El-Kordi, A., et al. 
2013 Development of an autism severity score for mice using Nlgn4 null mutants as a construct-valid model of heritable monogenic autism. Behav Brain Res 251:41-9.

Elsabbagh, M., et al.

2012 Global prevalence of autism and other pervasive developmental disorders. Autism Res 5(3):160-79.

Etherton, M. R., et al.

2009 Mouse neurexin-1alpha deletion causes correlated electrophysiological and behavioral changes consistent with cognitive impairments. Proc Natl Acad Sci U S A 106(42):17998-8003.

Flicek, P., et al.

2013 Ensembl 2013. Nucleic Acids Res 41(Database issue):D48-55.

Fombonne, E.

2003 Epidemiological surveys of autism and other pervasive developmental disorders: an update. J Autism Dev Disord 33(4):365-82.

Fond, G., et al.

2013 Effectiveness and tolerance of anti-inflammatory drugs' add-on therapy in major mental disorders: a systematic qualitative review. Acta Psychiatr Scand.

Frazier, T. W., et al.

2010 Autism spectrum disorders as a qualitatively distinct category from typical behavior in a large, clinically ascertained sample. Assessment 17(3):308-20.

Freitag, C. M.

2007 The genetics of autistic disorders and its clinical relevance: a review of the literature. Mol Psychiatry 12(1):2-22.

Fromer, M., et al.

2014 De novo mutations in schizophrenia implicate synaptic networks. Nature 506(7487):179-84.

Gailey, D. A., R. C. Lacaillade, and J. C. Hall

1986 Chemosensory elements of courtship in normal and mutant, olfaction-deficient Drosophila melanogaster. Behav Genet 16(3):375-405.

Georgiades, S., et al.

2013 Investigating phenotypic heterogeneity in children with autism spectrum disorder: a factor mixture modeling approach. J Child Psychol Psychiatry 54(2):206-15.

Georgiades, S., et al.

2011 Phenotypic overlap between core diagnostic features and emotional/behavioral problems in preschool children with autism spectrum disorder. J Autism Dev Disord 41(10):1321-9.

Georgiades, S., et al.

2007 Structure of the autism symptom phenotype: A proposed multidimensional model. J Am Acad Child Adolesc Psychiatry 46(2):18896.

Geschwind, D. H.

2009 Advances in autism. Annu Rev Med 60:367-80.

Gilbert, M., et al. 
2001 Neuroligin 3 is a vertebrate gliotactin expressed in the olfactory ensheathing glia, a growth-promoting class of macroglia. Glia 34(3):15164.

Gillberg, C.

1998 Chromosomal disorders and autism. J Autism Dev Disord 28(5):415-25.

Gleason, J. M.

2005 Mutations and natural genetic variation in the courtship song of Drosophila. Behav Genet 35(3):265-77.

Graf, E. R., et al.

2004 Neurexins induce differentiation of GABA and glutamate postsynaptic specializations via neuroligins. Cell 119(7):1013-26.

Grayton, H. M., et al.

2013 Altered social behaviours in neurexin 1alpha knockout mice resemble core symptoms in neurodevelopmental disorders. PLoS One 8(6):e67114.

Greenspan, R. J., and J. F. Ferveur 2000 Courtship in Drosophila. Annu Rev Genet 34:205-232.

Grube, S., et al.

2011 A CAG repeat polymorphism of KCNN3 predicts SK3 channel function and cognitive performance in schizophrenia. EMBO Mol Med 3(6):309-19.

Hahn, N., et al.

2013 Monogenic heritable autism gene neuroligin impacts Drosophila social behaviour. Behav Brain Res 252:450-7.

Hamilton, M.

1967 Development of a rating scale for primary depressive illness. The British journal of social and clinical psychology 6(4):278-96.

Han, K., et al.

2013 SHANK3 overexpression causes manic-like behaviour with unique pharmacogenetic properties. Nature 503(7474):72-7.

HapMapConsortium., International 2003 The International HapMap Project. Nature 426(6968):789-96.

Harrison, P. J., and S. L. Eastwood 1998 Preferential involvement of excitatory neurons in medial temporal Hayaishi, 0. lobe in schizophrenia. Lancet 352(9141):1669-73.

1999 Prostaglandin D2 and sleep--a molecular genetic approach. Journal of sleep research 8 Suppl 1:60-4.

Hoon, M., et al.

2011 Neuroligin-4 is localized to glycinergic postsynapses and regulates inhibition in the retina. Proc Natl Acad Sci U S A 108(7):3053-8.

Ichtchenko, K., et al.

1995 Neuroligin 1: a splice site-specific ligand for beta-neurexins. Cell 81(3):435-43.

Ichtchenko, K., T. Nguyen, and T. C. Sudhof 1996 Structures, alternative splicing, and neurexin binding of multiple neuroligins. J Biol Chem 271(5):2676-82.

IMGSAC 
2001 A genomewide screen for autism: strong evidence for linkage to chromosomes 2q, 7q, and 16p. Am J Hum Genet 69(3):570-81.

Irie, M., et al.

1997 Binding of neuroligins to PSD-95. Science 277(5331):1511-5.

Jamain, S., et al.

2003 Mutations of the X-linked genes encoding neuroligins NLGN3 and NLGN4 are associated with autism. Nat Genet 34(1):27-9.

Jamain, S., et al.

2008 Reduced social interaction and ultrasonic communication in a mouse model of monogenic heritable autism. Proc Natl Acad Sci U S A 105(5):1710-5.

Juven-Gershon, T., and J. T. Kadonaga

2010 Regulation of gene expression via the core promoter and the basal transcriptional machinery. Dev Biol 339(2):225-9.

Kamp-Becker, I., et al.

2009 Dimensional structure of the autism phenotype: relations between early development and current presentation. J Autism Dev Disord 39(4):557-71.

Kanner, L.

1943 Autistic Disturbances of Affective Contact. Nervous Child 2(3):217250.

Karlsgodt, K. H., et al.

2008 Developmental disruptions in neural connectivity in the pathophysiology of schizophrenia. Dev Psychopathol 20(4):1297-327.

Kay, S. R., A. Fiszbein, and L. A. Opler

1987 The positive and negative syndrome scale (PANSS) for schizophrenia. Schizophrenia bulletin 13(2):261-76.

Kent, W. J., et al.

2002 The human genome browser at UCSC. Genome Res 12(6):9961006.

Kim, H. G., et al.

2008 Disruption of neurexin 1 associated with autism spectrum disorder. Am J Hum Genet 82(1):199-207.

Kirov, G., et al.

2008 Comparative genome hybridization suggests a role for NRXN1 and APBA2 in schizophrenia. Hum Mol Genet 17(3):458-65.

Knable, M. B., et al.

2004 Molecular abnormalities of the hippocampus in severe psychiatric illness: postmortem findings from the Stanley Neuropathology Consortium. Mol Psychiatry 9(6):609-20, 544.

Knight, D., W. Xie, and G. L. Boulianne

2011 Neurexins and neuroligins: recent insights from invertebrates. Molecular neurobiology 44(3):426-440.

Knott, C., G. Stern, and G. P. Wilkin 2000 Inflammatory regulators in Parkinson's disease: iNOS, lipocortin-1, and cyclooxygenases- 1 and -2 . Molecular and cellular neurosciences 16(6):724-39.

Komoto, J., et al. 
2004 Crystal structure of human prostaglandin F synthase (AKR1C3). Biochemistry 43(8):2188-98.

Kramlinger, K. G., and R. M. Post

1996 Ultra-rapid and ultradian cycling in bipolar affective illness. The British journal of psychiatry : the journal of mental science 168(3):31423.

Krueger, D. D., et al.

2012 The role of neurexins and neuroligins in the formation, maturation, and function of vertebrate synapses. Curr Opin Neurobiol 22(3):412-22.

Kupka, R. W., et al.

2003 Rapid and non-rapid cycling bipolar disorder: a meta-analysis of clinical studies. J Clin Psychiatry 64(12):1483-94.

Laarakker, M. C., et al.

2012 Sex-dependent novelty response in neurexin-1alpha mutant mice. PLoS One 7(2):e31503.

Laflamme, N., S. Lacroix, and S. Rivest

1999 An essential role of interleukin-1beta in mediating NF-kappaB activity and COX-2 transcription in cells of the blood-brain barrier in response to a systemic and localized inflammation but not during endotoxemia. J Neurosci 19(24):10923-30.

Laumonnier, F., et al.

2004 X-linked mental retardation and autism are associated with a mutation in the NLGN4 gene, a member of the neuroligin family. Am J Hum Genet 74(3):552-7.

Leboyer, M., et al.

2012 Can bipolar disorder be viewed as a multi-system inflammatory disease? J Affect Disord 141(1):1-10.

Levinson, J. N., et al.

2010 Postsynaptic scaffolding molecules modulate the localization of Li, J., et al. neuroligins. Neuroscience 165(3):782-93.

2007 Crucial role of Drosophila neurexin in proper active zone apposition to postsynaptic densities, synaptic growth, and synaptic transmission. Neuron 55(5):741-55.

Lichtenstein, P., et al.

2010 The genetics of autism spectrum disorders and related neuropsychiatric disorders in childhood. Am J Psychiatry 167(11):135763.

Lin, H. K., et al.

1997 Expression and characterization of recombinant type 23 alphahydroxysteroid dehydrogenase (HSD) from human prostate: demonstration of bifunctional 3 alpha/17 beta-HSD activity and cellular distribution. Mol Endocrinol 11(13):1971-84.

Liston, T. E., and L. J. Roberts, 2nd

1985 Transformation of prostaglandin D2 to 9 alpha, 11 beta-(15S)trihydroxyprosta-(5Z,13E)-dien-1-oic acid (9 alpha, 11 betaprostaglandin F2): a unique biologically active prostaglandin produced enzymatically in vivo in humans. Proc Natl Acad Sci U S A 82(18):6030-4.

Lord, C., et al. 
2000 The autism diagnostic observation schedule-generic: a standard measure of social and communication deficits associated with the spectrum of autism. J Autism Dev Disord 30(3):205-23.

Lord, C., M. Rutter, and A. Le Couteur

1994 Autism Diagnostic Interview-Revised: a revised version of a diagnostic interview for caregivers of individuals with possible pervasive developmental disorders. J Autism Dev Disord 24(5):659-85.

Losh, M., et al.

2008 Defining key features of the broad autism phenotype: a comparison across parents of multiple- and single-incidence autism families. Am J Med Genet B Neuropsychiatr Genet 147B(4):424-33.

Maestrini, E., et al.

2010 High-density SNP association study and copy number variation analysis of the AUTS1 and AUTS5 loci implicate the IMMP2L-DOCK4 gene region in autism susceptibility. Mol Psychiatry 15(9):954-68.

Malhotra, D., and J. Sebat

2012 CNVs: harbingers of a rare variant revolution in psychiatric genetics. Cell 148(6):1223-41.

Mantel, A., et al.

2012 Aldo-keto reductase 1C3 is expressed in differentiated human epidermis, affects keratinocyte differentiation, and is upregulated in atopic dermatitis. J Invest Dermatol 132(4):1103-10.

Marshall, C. R., et al.

2008 Structural variation of chromosomes in autism spectrum disorder. Am J Hum Genet 82(2):477-88.

Matsumura, H., et al.

1994 Prostaglandin D2-sensitive, sleep-promoting zone defined in the ventral surface of the rostral basal forebrain. Proceedings of the National Academy of Sciences of the United States of America 91(25):11998-2002.

Matsuura, K., et al.

1998 Identification of a principal mRNA species for human 3alphahydroxysteroid dehydrogenase isoform (AKR1C3) that exhibits high prostaglandin D2 11-ketoreductase activity. J Biochem 124(5):940-6.

Meyer, G., et al.

2004 The complexity of PDZ domain-mediated interactions at glutamatergic synapses: a case study on neuroligin. Neuropharmacology 47(5):724-33.

Miles, J. H., et al.

2005 Essential versus complex autism: definition of fundamental prognostic subtypes. Am J Med Genet A 135(2):171-80.

Missler, M., and T. C. Sudhof

1998 Neurexins: three genes and 1001 products. Trends in genetics : TIG 14(1):20-6.

Morrow, E. M., et al.

2008 Identifying autism loci and genes by tracing recent shared ancestry. Science 321(5886):218-23.

Muller, N., et al. 
2006 The cyclooxygenase-2 inhibitor celecoxib has therapeutic effects in major depression: results of a double-blind, randomized, placebo controlled, add-on pilot study to reboxetine. Mol Psychiatry 11(7):680-4.

Munson, J., et al.

2008 Evidence for latent classes of IQ in young children with autism spectrum disorder. Am J Ment Retard 113(6):439-52.

Murphy, M., et al.

2000 Personality traits of the relatives of autistic probands. Psychol Med 30(6):1411-24.

Nakanishi, M., and D. W. Rosenberg

2013 Multifaceted roles of PGE2 in inflammation and cancer. Semin Immunopathol 35(2):123-37.

Nguyen, T., and T. C. Sudhof

1997 Binding properties of neuroligin 1 and neurexin 1beta reveal function as heterophilic cell adhesion molecules. J Biol Chem 272(41):26032-9.

O'Hara, B. F., et al.

1999 Gene expression in the brain across the hibernation cycle. J Neurosci 19(10):3781-90.

Onoe, H., et al.

1988 Prostaglandin D2, a cerebral sleep-inducing substance in monkeys. Proceedings of the National Academy of Sciences of the United States of America 85(11):4082-6.

Owen, M. J., et al.

2011 Neurodevelopmental hypothesis of schizophrenia. The British journal of psychiatry : the journal of mental science 198(3):173-5.

Ozonoff, S., et al.

2008 The onset of autism: patterns of symptom emergence in the first years of life. Autism Res 1(6):320-8.

Pampanos, A., et al.

2009 A substitution involving the NLGN4 gene associated with autistic behavior in the Greek population. Genet Test Mol Biomarkers 13(5):6115.

Papadopoulos, T., et al.

2008 Collybistin is required for both the formation and maintenance of GABAergic postsynapses in the hippocampus. Molecular and cellular

Peca, J., et al. neurosciences 39(2):161-9.

2011 Shank3 mutant mice display autistic-like behaviours and striatal dysfunction. Nature 472(7344):437-42.

Pichon, X., et al.

2012 RNA binding protein/RNA element interactions and the control of translation. Curr Protein Pept Sci 13(4):294-304.

Piven, J., et al.

1997 Broader autism phenotype: evidence from a family history study of multiple-incidence autism families. Am J Psychiatry 154(2):185-90.

Plante, D. T., and J. W. Winkelman

2008 Sleep disturbance in bipolar disorder: therapeutic implications. Am J Psychiatry 165(7):830-43. 
Popov, A. V., et al.

2005 The Role of the Flabellar and Ellipsoid Bodies of the Central Complex of the Brain of Drosophila Melanogaster in the Control of Courtship Behavior and Communicative Sound Production in Males. Neuroscience and Behavioral Physiology 35(7):741-750.

Radyushkin, K., et al.

2009 Neuroligin-3-deficient mice: model of a monogenic heritable form of autism with an olfactory deficit. Genes Brain Behav 8(4):416-25.

Ribbe, K., et al.

2010 The cross-sectional GRAS sample: a comprehensive phenotypical data collection of schizophrenic patients. BMC psychiatry 10:91.

Ritvo, E. R., et al.

1989 The UCLA-University of Utah epidemiologic survey of autism: prevalence. The American journal of psychiatry 146(2):194-9.

Rosenberg, R. E., et al.

2009 Characteristics and concordance of autism spectrum disorders among 277 twin pairs. Arch Pediatr Adolesc Med 163(10):907-14.

Rubenstein, J. L., and M. M. Merzenich

2003 Model of autism: increased ratio of excitation/inhibition in key neural systems. Genes Brain Behav 2(5):255-67.

Rudenko, G., et al.

1999 The structure of the ligand-binding domain of neurexin Ibeta: regulation of LNS domain function by alternative splicing. Cell 99(1):93101.

Rujescu, D., et al.

2009 Disruption of the neurexin 1 gene is associated with schizophrenia. Hum Mol Genet 18(5):988-96.

Sarasua, S. M., et al.

2011 Association between deletion size and important phenotypes expands the genomic region of interest in Phelan-McDermid syndrome (22q13 deletion syndrome). J Med Genet 48(11):761-6.

Scheiffele, P., et al.

2000 Neuroligin expressed in nonneuronal cells triggers presynaptic development in contacting axons. Cell 101(6):657-69.

Sebat, J., et al.

2007 Strong association of de novo copy number mutations with autism. Science 316(5823):445-9.

Sebat, J., D. L. Levy, and S. E. McCarthy

2009 Rare structural variants in schizophrenia: one disorder, multiple mutations; one mutation, multiple disorders. Trends in genetics : TIG 25(12):528-35.

Shao, Y., et al.

2002 Genomic screen and follow-up analysis for autistic disorder. Am J Med Genet 114(1):99-105.

Sherry, S. T., et al.

2001 dbSNP: the NCBI database of genetic variation. Nucleic Acids Res 29(1):308-11.

Shinawi, M., et al. 
2009 The Xp contiguous deletion syndrome and autism. Am J Med Genet A 149A(6):1138-48.

Smith, W. L., D. L. DeWitt, and R. M. Garavito

2000 Cyclooxygenases: structural, cellular, and molecular biology. Annu Rev Biochem 69:145-82.

Snow, A. V., L. Lecavalier, and C. Houts 2009 The structure of the Autism Diagnostic Interview-Revised: diagnostic and phenotypic implications. J Child Psychol Psychiatry 50(6):734-42.

Song, J. Y., et al.

1999 Neuroligin 1 is a postsynaptic cell-adhesion molecule of excitatory synapses. Proc Natl Acad Sci U S A 96(3):1100-5.

Spence, S. J., and M. T. Schneider

2009 The role of epilepsy and epileptiform EEGs in autism spectrum disorders. Pediatr Res 65(6):599-606.

Stefansson, H., et al.

2014 CNVs conferring risk of autism or schizophrenia affect cognition in controls. Nature 505(7483):361-6.

Stephan, K. E., T. Baldeweg, and K. J. Friston

2006 Synaptic plasticity and dysconnection in schizophrenia. Biological psychiatry 59(10):929-39.

Sun, M., et al.

2011 Neuroligin 2 is required for synapse development and function at the Drosophila neuromuscular junction. J Neurosci 31(2):687-99.

Sun, S., and J. Heitman

2012 Should Y stay or should Y go: the evolution of non-recombining sex chromosomes. Bioessays 34(11):938-42.

Szatmari, P., et al.

2000 The familial aggregation of the lesser variant in biological and nonbiological relatives of PDD probands: a family history study. J Child Psychol Psychiatry 41(5):579-86.

Szatmari, P., et al.

2007 Mapping autism risk loci using genetic linkage and chromosomal rearrangements. Nat Genet 39(3):319-28.

Tabuchi, K., et al.

2007 A neuroligin-3 mutation implicated in autism increases inhibitory synaptic transmission in mice. Science 318(5847):71-6.

Tager-Flusberg, H., and R. M. Joseph

2003 Identifying neurocognitive phenotypes in autism. Philos Trans R Soc Lond B Biol Sci 358(1430):303-14.

Tai, H. H., et al.

2006 NAD+-linked 15-hydroxyprostaglandin dehydrogenase: structure and biological functions. Curr Pharm Des 12(8):955-62.

Takahata, R., et al.

1996 Seasonal variation in levels of prostaglandins D2, E2 and F2(alpha) in the brain of a mammalian hibernator, the Asian chipmunk. Prostaglandins, leukotrienes, and essential fatty acids 54(1):77-81.

Taniai, H., et al. 
2008 Genetic influences on the broad spectrum of autism: study of proband-ascertained twins. Am J Med Genet B Neuropsychiatr Genet 147B(6):844-9.

Tauber, Eran, and Daniel F. Eberl

2003 Acoustic communication in Drosophila. Behavioural Processes 64(2):197-210.

Toal, F., et al.

2009 Psychosis and autism: magnetic resonance imaging study of brain anatomy. The British journal of psychiatry : the journal of mental science 194(5):418-25.

Tobaben, S., T. C. Sudhof, and B. Stahl

2000 The G protein-coupled receptor CL1 interacts directly with proteins of the Shank family. J Biol Chem 275(46):36204-10.

Tondo, L., J. Hennen, and R. J. Baldessarini

2003 Rapid-cycling bipolar disorder: effects of long-term treatments. Acta Psychiatr Scand 108(1):4-14.

Ullrich, B., Y. A. Ushkaryov, and T. C. Sudhof

1995 Cartography of neurexins: more than 1000 isoforms generated by alternative splicing and expressed in distinct subsets of neurons. Neuron 14(3):497-507.

Urade, Y., and 0. Hayaishi

2000 Biochemical, structural, genetic, physiological, and pathophysiological features of lipocalin-type prostaglandin D synthase. Biochim Biophys Acta 1482(1-2):259-71.

2011 Prostaglandin D2 and sleep/wake regulation. Sleep medicine reviews 15(6):411-8.

Ushkaryov, Y. A., et al.

1994 Conserved domain structure of beta-neurexins. Unusual cleaved signal sequences in receptor-like neuronal cell-surface proteins. J Biol Chem 269(16):11987-92.

Ushkaryov, Y. A., et al.

1992 Neurexins: synaptic cell surface proteins related to the alphalatrotoxin receptor and laminin. Science 257(5066):50-6.

Ushkaryov, Y. A., and T. C. Sudhof

1993 Neurexin III alpha: extensive alternative splicing generates membrane-bound and soluble forms. Proc Natl Acad Sci U S A 90(14):6410-4.

van Lang, N. D., et al.

2006 Structural equation analysis of a hypothesised symptom model in the autism spectrum. J Child Psychol Psychiatry 47(1):37-44.

Vane, J. R., Y. S. Bakhle, and R. M. Botting

1998 CYCLOOXYGENASES 1 AND 2. Annual Review of Pharmacology and Toxicology 38(1):97-120.

Varoqueaux, F., et al.

2006 Neuroligins determine synapse maturation and function. Neuron 51(6):741-54.

Varoqueaux, F., S. Jamain, and N. Brose 
2004 Neuroligin 2 is exclusively localized to inhibitory synapses. Eur J Cell Biol 83(9):449-56.

von Schilcher, Florian

1976 The role of auditory stimuli in the courtship of Drosophila melanogaster. Animal Behaviour 24(1):18-26.

Walsh, T., et al.

2008 Rare structural variants disrupt multiple genes in neurodevelopmental pathways in schizophrenia. Science 320(5875):53943.

Wang, K., et al.

2009 Common genetic variants on 5p14.1 associate with autism spectrum disorders. Nature 459(7246):528-33.

Watanabe, K., et al.

1986 Stereospecific conversion of prostaglandin D2 to (5Z,13E)-(15S)-9 alpha-11 beta,15-trihydroxyprosta-5,13-dien-1-oic acid ( 9 alpha,11 betaprostaglandin F2) and of prostaglandin $\mathrm{H} 2$ to prostaglandin F2 alpha by bovine lung prostaglandin $F$ synthase. Proc Natl Acad Sci U S A 83(6):1583-7.

Weiss, L. A., et al.

2009 A genome-wide linkage and association scan reveals novel loci for autism. Nature 461(7265):802-8.

Wentz, E., et al.

2013 A case report of two male siblings with autism and duplication of Xq13-q21, a region including three genes predisposing for autism. Eur Child Adolesc Psychiatry.

Wheeler, D. A., W. L. Fields, and J. C. Hall

1988 Spectral analysis of Drosophila courtship songs: D. melanogaster, D. simulans, and their interspecific hybrid. Behav Genet 18(6):675-703.

Wing, L., and J. Gould

1979 Severe impairments of social interaction and associated abnormalities in children: epidemiology and classification. J Autism Dev Disord 9(1):11-29.

Yamagata, K., et al.

1993 Expression of a mitogen-inducible cyclooxygenase in brain neurons: regulation by synaptic activity and glucocorticoids. Neuron 11(2):371-86.

Yan, J., et al.

2008 Analysis of the neuroligin 4Y gene in patients with autism. Psychiatr Genet 18(4):204-7.

Yin, D. M., et al.

2012 Synaptic dysfunction in schizophrenia. Adv Exp Med Biol 970:493516.

Yizhar, O., et al.

2011 Neocortical excitation/inhibition balance in information processing and social dysfunction. Nature 477(7363):171-8.

Young, R. C., et al.

1978 A rating scale for mania: reliability, validity and sensitivity. The

British journal of psychiatry : the journal of mental science 133:429-35.

Zeev-Ben-Mordehai, T., et al. 
2003 The intracellular domain of the Drosophila cholinesterase-like neural adhesion protein, gliotactin, is natively unfolded. Proteins 53(3):758-67.

Zeng, X., et al.

2007 Neurexin-1 is required for synapse formation and larvae associative learning in Drosophila. FEBS Lett 581(13):2509-16. 


\section{Appendix}

\subsection{First publication in press}

\section{Brief Report}

\section{A role for prostaglandins in rapid cycling suggested by episode-specific gene expression shifts in peripheral blood mononuclear cells: a preliminary report}

\begin{abstract}
Gurvich A, Begemann M, Dahm L, Sargin D, Miskowiak K, Ehrenreich $\mathrm{H}$. A role for prostaglandins in rapid cycling suggested by episodespecific gene expression shifts in peripheral blood mononuclear cells: a preliminary report.

Bipolar Disord 2014: 00: 000-000. (C) 2014 John Wiley \& Sons A/S. Published by John Wiley \& Sons Ltd.

Objectives: Over $12 \%$ of patients with bipolar disorder exhibit rapid cycling. The underlying biological mechanisms of this extreme form of bipolar disease are still unknown. This study aimed at replicating and extending findings of our previously published case report, where an involvement of prostaglandin synthesis-related genes in rapid cycling was first proposed.
\end{abstract}

Methods: Psychopathological follow-up of the reported case was performed under cessation of celecoxib treatment. In a prospective observational study, patients with bipolar disorder $(n=47$; of these, four had rapid cycling) or with monopolar depression $(n=97)$ were recruited over a period of three years. Repeated psychopathology measurements were conducted using standard instruments. Peripheral blood mononuclear cells (PBMC) were obtained during as many consecutive episodes as possible and processed for mRNA isolation and quantitative real-time reverse transcriptase polymerase chain reaction for prostaglandin D2 synthase ( $P T G D S)$, aldo-ketoreductase family 1 , member $\mathrm{C} 3(A K R 1 C 3), C O X 2_{P A N}$, prostaglandin-endoperoxide synthase 2 (PTGS2), and purinergic receptor $\mathrm{P} 2 \mathrm{X}$, ligand-gated ion channel 7 $4(P 2 R X 7)$.

Results: The follow-up of our original case of a patient with rapid $\mathbf{5}$ cycling who had shown impressive psychopathological improvement under celecoxib revealed complete loss of this effect upon discontinuation of the COX2 inhibitor. Episode-specific gene expression measurements in PBMC of four newly recruited rapid cycling patients confirmed the higher expression of $P T G D S$ in depressive compared to manic phases. Additionally, higher relative expression of $P T G S 2$ $C O X 2_{P A N}$ was found. No comparable alterations were observable in samples available from the remaining 43 patients with bipolar disorder and the 97 monopolar depressed patients, emphasizing the advantages of the rapid cycling condition with its rapid and frequent shifts for identification of gene expression changes.

Conclusions: This study supports a role for prostaglandins in rapid cycling and advocates the cyclooxygenase cascade as a treatment target in this condition.

\section{Artem Gurvich $^{\mathrm{a}, *}$, Martin} Begemann $^{\mathrm{a}, \mathrm{b} *}$, Liane Dahm ${ }^{\mathrm{a}}$, Derya Sargin ${ }^{\mathrm{a}}$, Kamilla Miskowiak ${ }^{\mathrm{c}}$ and Hannelore Ehrenreich ${ }^{\mathrm{a}, \mathrm{b}}$

${ }^{a}$ Clinical Neuroscience, Max Planck Institute of Experimental Medicine, ${ }^{\mathrm{D}} \mathrm{DFG}$ Center for Nanoscale Microscopy and Molecular Physiology of the Brain (CNMPB), Göttingen, Germany, ${ }^{\circ}$ Department of Psychiatry. Copenhagen Psychiatric Centre, Copenhagen ص University Hospital, Rigshospitalet, Denmark

\section{doi: 10.1111/bdi.12223}

Key words: aldo-ketoreductase family 1 , member C3 (AKR1C3) - bipolar disorder celecoxib - cyclooxygenase 2 (COX2) hibernation - monopolar depression purinergic receptor $P 2 X$, ligand-gated ion

6. channel 7 (P2RX7) - prostaglandin synthesis prostaglandin D2 synthase (PTGDS) prostaglandin-endoperoxide synthase 2 (PTGS2)

Received 11 March 2014, revised and accepted [ for publication 16 May 2014

\section{Corresponding author:}

Professor Hannelore Ehrenreich, M.D., D.V.M. Clinical Neuroscience

Max Planck Institute of Experimental Medicine

s Göttingen ????

Germany

Fax: +49-551-3899670

E-mail: ehrenreich@em.mpg.de

"These authors contributed equally to this project. 


\section{Gurvich et al.}

Rapid cycling constitutes a severe and disabling 7 subgroup of bipolar affective disorders, present in $>12 \%$ of the bipolar population (1). According to DSM-5 (2), the essential feature of rapid cycling bipolar disorder is the occurrence of at least four mood episodes during the previous 12 months, These episodes can occur in any combination and order. They must meet both the duration and symptom number criteria for a major depressive, manic, or hypomanic episode and be demarcated by either a period of full remission or a switch to an episode of the opposite polarity. Manic and hypomanic episodes are counted as being on the same pole. Except for the fact that they occur more frequently, the episodes are no different from those seen in a non-rapid cycling pattern. The underlying etiology and pathogenesis of bipolar disease in general and of rapid cycling in particular are still completely unknown.

We previously described the case of a female 8 patient with pronounced rapid cycling, whom we have followed up for $>20$ years (3). In this case, we uncovered consistent episode-specific expression shifts in genes encoding determinants of prostaglandin synthesis, prostaglandin D2 synthase $(P T G D S)$ and aldo-ketoreductase family 1 , member C3 (AKR1C3). Since PTGDS and prostaglandins are associated with hibernation $(4,5)$ as an established cycling condition sharing behavioral and autonomic features with bipolar disease, we proposed a pathological hibernation hypothesis of rapid cycling (3). The unavailability of effective treatment options in this severe condition prompted us to initiate a compassionate use approach, employing a cyclooxygenase 2 (COX2) inhibitor. This approach was independently supported by recent treatment trials in affective disorders using celecoxib as an anti-inflammatory strategy $(6,7)$. In our case, celecoxib indeed led to considerable attenuation of affective symptom severity, both depressive and manic (3).

Here we provide a brief follow-up of our original 9 female patient with regard to psychopathology upon cessation of celecoxib treatment. Encouraged by these findings, we initiated the present prospective study to (i) extend the original peripheral blood mononuclear cell (PBMC) gene expression

10 findings to four other rapid cycling patients by including PTGDS, $A K R 1 C 3, C O X 2_{P A N}$, and prostaglandin-endoperoxide synthase 2 (PTGS2) in the analysis and adding purinergic receptor $\mathrm{P} 2 \mathrm{X}$, ligand-gated ion channel $7(P 2 R \times 7)$ as a prostaglandin-unrelated control gene; and (ii) integrate PBMC samples of a number of bipolar and monopolar affective patients without rapid cycling diagnosis into the analyses.

\section{Materials and methods}

Patients

The present prospective study was approved by the ethical committee of the Georg August University of Göttingen. All patients gave informed consent. 12 Recruitment took place from 2010 to 2013. From a total of seven patients (three male and four female) who were identified during the recruitment period as rapid cyclers, four patients (three male and one female) agreed to return for regular follow-up examinations and could thus be included. Their sociodemographic and clinical characteristics are shown in Table 1. Moreover, 43 patients with bipolar affective disorder (male/female $=24 / 19$; mean age: $50.0 \pm 12.1$ years; mean age at disease onset: $34.5 \pm 11.6$ years; mean number of phases/ year: $0.9 \pm 0.6$ ) as well as 97 monopolar depressed patients $($ male $/$ female $=42 / 55$; mean age: $49.4 \pm$ 15.0 years; mean age at disease onset: $41.0 \pm$ 15.7 years; mean number of phases/year: $1.2 \pm 1.3$ ) were recruited. All patients either kept their pre-existing medication during the study or had treatment performed as usual. There was no $\mathbf{1 3}$ particular systematic intervention in this study.

\section{Psychopathology rating}

Psychopathology was assessed using standard instruments: the Hamilton Depression Rating Scale (HAM-D) (8), Beck Depression Inventory (BDI) (9), Young Mania Rating Scale (YMRS) (10), and Positive and Negative Syndrome Scale (PANSS) (11).

\section{Procedures}

Isolation of PBMC. Blood samples were obtained via phlebotomy into citrate phosphate dextrose adenine (CPDA) vials (Sarstedt, Germany) 14 between 7:00 a.m. and 8:00 a.m. after an overnight fast. PBMC were collected by applying the standard Ficoll-Paque Plus isolation procedure (GE Healthcare, Munich, Germany). For RNA isolation, the miRNeasy Mini Kit (Qiagen, Hilden, Germany) was used. The cDNA was transcribed from $1 \mu \mathrm{g}$ of total RNA with a mixture of oligo$\mathrm{dT}$ and hexamer primers, dNTPs, DTT and $200 \mathrm{U}$ SuperScriptIII (Life Technologies GmbH, Darmstadt, Germany). The mixture was incubated for $10 \mathrm{~min}$ at $25^{\circ} \mathrm{C}, 45 \mathrm{~min}$ at $50^{\circ} \mathrm{C}$, and $45 \mathrm{~min}$ at $55^{\circ} \mathrm{C}$.

Quantitative real-time reverse transcriptasepolymerase chain reaction $(q R T-P C R)$. For the qRT-PCR, the cDNA was diluted $1: 25$ in a $10-\mu 1$ 
Prostaglandins in rapid cycling

Table 1. Sociodemographic and clinical characteristics of four patients with rapid cycling bipolar disorder

\begin{tabular}{|c|c|c|c|c|c|c|c|c|}
\hline & \multicolumn{2}{|c|}{ Patient 1 (male) } & \multicolumn{2}{|c|}{ Patient 2 (male) } & \multicolumn{2}{|c|}{ Patent 3 (male) } & \multicolumn{2}{|c|}{ Patient 4 (female) } \\
\hline \multicolumn{9}{|c|}{ Sociodemographic data } \\
\hline Year of birth & \multicolumn{2}{|l|}{1958} & \multicolumn{2}{|l|}{1962} & \multicolumn{2}{|l|}{1941} & \multicolumn{2}{|l|}{1959} \\
\hline Education, years ${ }^{a}$ & \multicolumn{2}{|c|}{$10+3.5$} & \multicolumn{2}{|c|}{$9+3.5$} & \multicolumn{2}{|l|}{$13+6.0$} & \\
\hline Employment & \multicolumn{2}{|c|}{ Early retirement ${ }^{b}$} & \multicolumn{2}{|c|}{ Early retirement $^{c}$} & \multicolumn{2}{|c|}{ Early retirement ${ }^{\mathrm{b}}$} & \multicolumn{2}{|c|}{ Early retirement $^{d}$} \\
\hline Disease course & & & & & & & & \\
\hline Age of onset, years & \multicolumn{2}{|l|}{20} & \multicolumn{2}{|l|}{27} & \multicolumn{2}{|l|}{22} & \multicolumn{2}{|l|}{50} \\
\hline Phases/year & \multicolumn{2}{|l|}{$8-10$} & \multicolumn{2}{|l|}{45} & \multicolumn{2}{|l|}{$10-12$} & \multicolumn{2}{|l|}{$12-16$} \\
\hline \multicolumn{9}{|l|}{ Psychopathology } \\
\hline HAM-D score & $0-5$ & $8-28$ & $0-5$ & $13-16$ & $5-9$ & $8-33$ & $0-2$ & $12-17$ \\
\hline BDI score & n.p. & $20-36$ & 8 & n.p. & $7-25$ & $8-48$ & $7-10$ & $13-17$ \\
\hline YMRS score & $14-20$ & $0-1$ & $23-27$ & 0 & $9-26$ & 0 & $12-17$ & $0-1$ \\
\hline PANSS $_{\text {total }}$ score & $33-45$ & $31-53$ & 48 & $49-60$ & $39-55$ & $37-85$ & $38-43$ & 25-92 \\
\hline Suicidality & No & Thoughts & No & Thoughts & No & Thoughts & No & Thoughts \\
\hline \multicolumn{9}{|c|}{ Autonomic and physical signs } \\
\hline Phase & M & D & M & D & M & D & M & D \\
\hline Sleep & Less & Increase & Less & Increase & Less & Less & Less & Less \\
\hline Appetite & Increase & Loss & Loss & Loss & Increase & Loss & Increase & Loss \\
\hline Weight & No change & Loss & Gain & Loss & No change & Loss & No change & No change \\
\hline \multicolumn{9}{|l|}{ Medication } \\
\hline Present & \multirow{2}{*}{\multicolumn{2}{|c|}{$\begin{array}{l}\text { QTP, LTG, VAL, } \\
\text { L-thyroxin } \\
\text { Lithium }\end{array}$}} & \multirow{2}{*}{\multicolumn{2}{|c|}{$\begin{array}{l}\text { Perphenazine, LTG, VAL, } \\
\text { melperone, citalopram } \\
\text { Lithium, carbamazepine, } \\
\text { haloperidol, } \\
\text { levomepromazine, } \\
\text { benperidol, } \\
\text { olanzapine, clozapine, } \\
\text { phenobarbital, ECT }\end{array}$}} & \multirow{2}{*}{\multicolumn{2}{|c|}{$\begin{array}{l}\text { Venlafaxine, aripiprazole, } \\
\text { L-thyroxin } \\
\text { Lithium, bupropion, LTG, } \\
\text { VAL, QTP, lorazepam, ECT }\end{array}$}} & \multirow{2}{*}{\multicolumn{2}{|c|}{$\begin{array}{l}\text { Venlafaxine, QTP, } \\
\text { lithium, LTG, ECT } \\
\text { Lithium }\end{array}$}} \\
\hline Past & & & & & & & & \\
\hline
\end{tabular}

$\mathrm{BDI}=$ Beck Depression Inventory; $\mathrm{D}=$ depressed phase; $\mathrm{ECT}=$ electroconvulsive therapy; HAM-D = Hamilton Depression Rating Scale; $L T G=$ lamotrigine; $M=$ manic phase; $n . p$. = not performed; PANSS = Positive and Negative Syndrome Scale; $Q T P=$ quetiapine; $\mathrm{VAL}=$ valproate; $\mathrm{YMRS}=$ Young Mania Rating Scale.

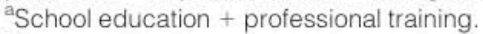

${ }^{\mathrm{b}} \mathrm{Age}=41$ years.

${ }^{\mathrm{C}} \mathrm{Age}=47$ years.

${ }^{\mathrm{d}} \mathrm{Age}=51$ years .

reaction mix, containing $5 \mu \mathrm{l}$ of SYBR Green (Life Technologies $\mathrm{GmbH}$ ) and 1 pmol of each primer. The following primer sequences were employed:

(i) PTGDS: 5' CGGCTCCTACAGCTACCG $3^{\prime}$ (forward); 5' CAGCGCGTACTGGTCGTA $3^{\prime}$ (reverse)

(ii) $A K R 1 C 3: 5^{\prime}$ CATTGGGGTGTCAAACTT CA $3^{\prime}$ (forward); 5' CCGGTTGAAATACGGATGAC $3^{\prime}$ (reverse)

(iii) $C O X 2_{P A N}: 5^{\prime}$ GAGCAGGCAGATGAAATACCAG $3^{\prime}$ (forward); 5' GTTGGAAGCACTC TATGGTGACA $3^{\prime}$ (reverse)

(iv) PTGS2: 5' GATCCCCAGGGCTCAAACAT $3^{\prime}$ (forward); 5' TCATATTTACGGTGAAACT CTGG $3^{\prime}$ (reverse) (v) $P 2 R X 7: 5^{\prime}$ AGGAAGAAGTGCGAGTCCAT 3' (forward); 5' CTGCTGGTTCACCATCCTAA $3^{\prime}$ (reverse)

(vi) GAPDH: 5' CTGACTTCAACAGCGA- 15 CACC $3^{\prime}$ (forward); 5' TGCTGTAGCCAAATTC GTTGT $3^{\prime}$ (reverse)

The samples were run with technical triplicates on the LightCycler480 system (Roche Diagnostics GmbH, Mannheim, Germany) with the following profile: pre-heating at $95^{\circ} \mathrm{C}$ for $10 \mathrm{~min} ; 45$ cycles of $95^{\circ} \mathrm{C}$ for $15 \mathrm{sec}$ and $60^{\circ} \mathrm{C}$ for $1 \mathrm{~min}$. Cycle threshold (CT) values were standardized to CT values of $G A P D H$. In order to control for intraand interplate variability due to technical reasons, a reference sample, containing cDNA from pooled 


\section{Gurvich et al.}

PBMC-derived mRNA from four healthy controls, was included at the beginning, in the middle, and at the end of each plate. The values standardized to $G A P D H$ were further standardized to the mean CT value of the reference sample in the respective plate.

\section{Statistical analyses}

All numerical results are presented as mean \pm standard deviation (SD) in the text and mean \pm standard error (SEM) in the figures. Student's $t$-test (two-tailed or one-tailed if applicable) was performed using GraphPad Prism version 5.04 for Windows (GraphPad Software, San Diego, CA, USA).

\section{Results}

In our previous rapid cycling case report, a significant decrease in the severity of depressive as well as manic symptoms was observed upon treatment with the COX2 inhibitor celecoxib (3). During a depressed phase, the patient decided to discontinue celecoxib treatment. This gave us the opportunity to monitor psychopathology upon discontinuation

17 of celecoxib (using HAM-D and YMRS). As illustrated in Figure 1A, values returned to pretreatment levels.

This finding further indicated a causal involvement of prostaglandins in rapid cycling and encouraged us to start the present prospective observational study, aiming to reproduce the expression shifts of prostaglandin-related genes in rapid cycling. In parallel, we included bipolar affective disorder and monopolar depressed patients. During the recruitment process, a total of seven rapid cycling patients (three male and four female) were identified, of whom four (three male and one female) agreed to return for frequent regular follow-up investigations including blood sampling. Patient characteristics of these four individuals, including sociodemographic, psychopathological and medication data, are given in Table 1 .

All four patients were receiving social security insurance after early retirement due to permanent disability. With at least four affective episodes per year, the diagnosis of rapid cycling was supported in all subjects. Mood stabilizers, antipsychotics, and antidepressant medication were given in previous years with very limited success.

In these patients, we explored the expression in PBMC of PTGDS and $A K R I C 3$, the two genes involved in prostaglandin synthesis which

20 were previously found to be regulated during

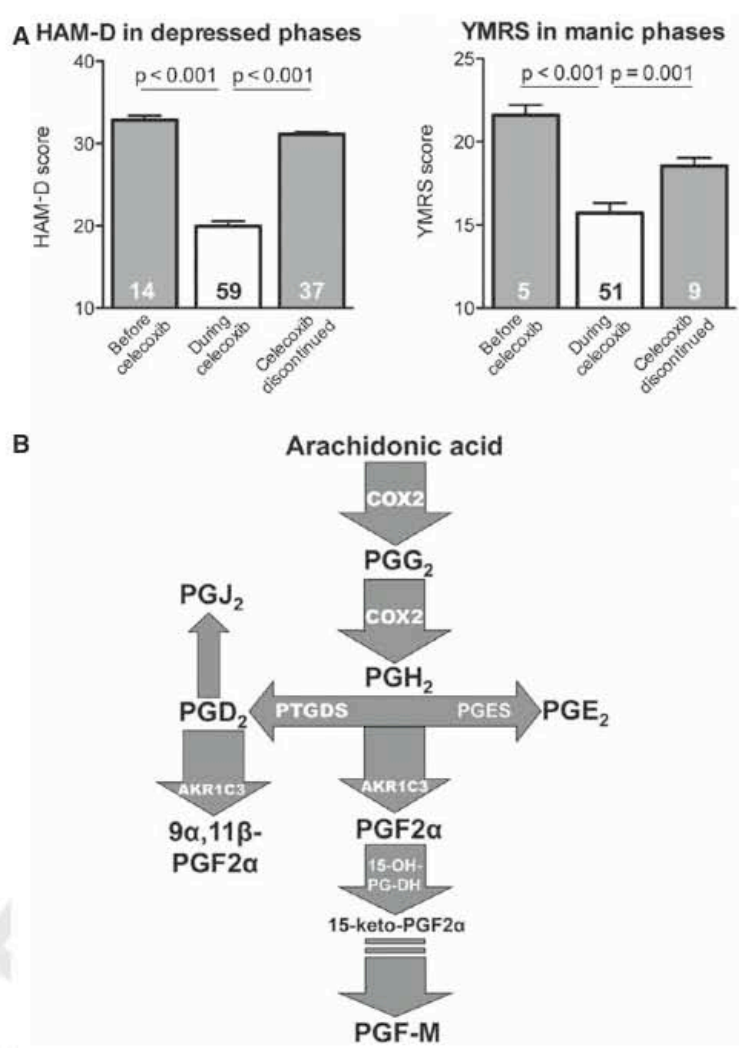

Fig. 1. (A) Psychopathology rating of a female patient with 18 severe rapid cycling before, during, and upon cessation of celecoxib treatment $(300 \mathrm{mg} /$ day $)$. Numbers in the columns denote the number of independent psychopathology ratings in the respective episodes. Mean \pm standard error is presented. (19 HAM-D $=$ Hamilton Depression Rating Scale; YMRS = Young Mania Rating Scale. (B) Prostaglandin synthesis pathway illustrating the position of the enzymes encoded by the genes of interest (34-37). COX2 = cyclooxygenase-2 (alias PTGS2 = prostaglandin-endoperoxide synthase 2$) ; \quad P G G 2=$ prostaglandin $\mathrm{G} 2 ; P G H 2=$ prostaglandin $\mathrm{H} 2 ; P G E 2=$ prostaglandin $\mathrm{E} 2 ; P G J 2=$ prostaglandin $\mathrm{J} 2 ; P G F 2 \alpha=$ prostaglandin $\mathrm{F} 2 \alpha ; P G D 2=$ prostaglandin D2; $P G F-M=$ prostaglandin F metabolites; PTGDS $=$ prostaglandin D2 synthase; $A K R 1 C 3=$ aldo-ketoreductase family 1 , member $\mathrm{C} 3$; $P G E S=$ prostaglandin E synthase; $15-O H-P G-D H=15$-hydroxyprostaglandin dehydrogenase.

episodes (Fig. 1B) (3). As a new central readout in prostaglandin synthesis-related genes, we additionally determined the ratio of $P T G S 2 / C O X 2$ $P A N$. COX2, better referred to as COX2PAN, has two splice variants, PTGS2 and COX2a, the latter having been identified in platelets $(12,13)$. To specifically address potential alterations of the brain-expressed PTGS2 $(14,15)$ in relation to $C O X 2_{P A N}$, we calculated their ratio. As a non-prostaglandin-related control gene, not indisputably associated with bipolar disorder (16-18), we utilized $P 2 R X 7$ encoding the P2X 21 purinoceptor 7 . 


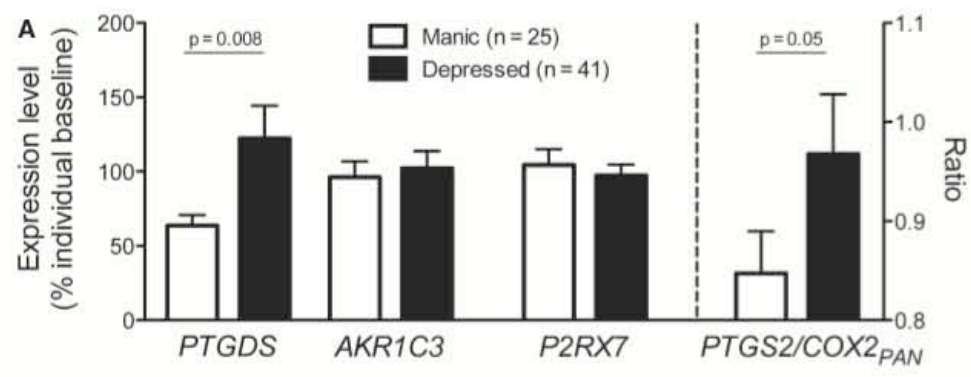

B Patient\#1

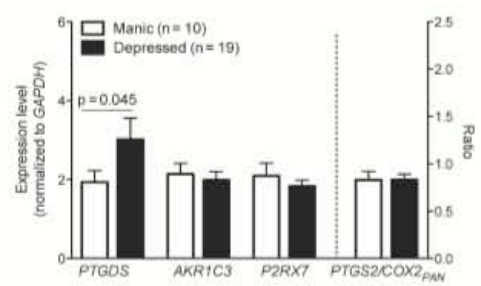

D Patient\#3

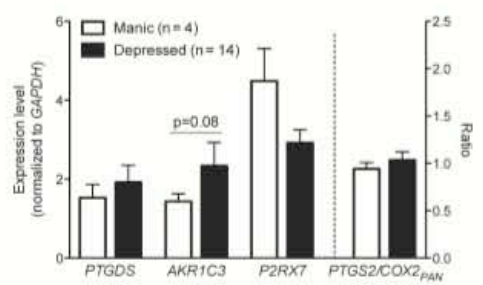

C Patient\#2

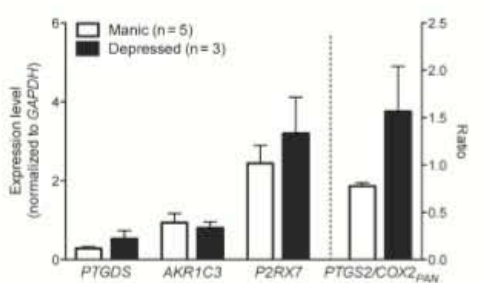

E Patient\#4

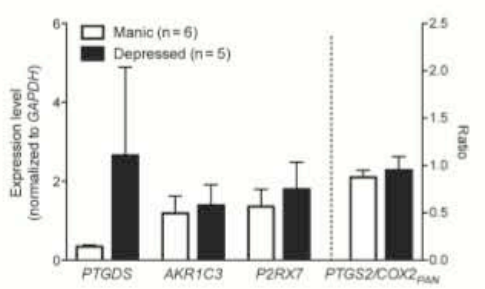

Fig. 2. Episode-specific gene expression in four rapid cycling patients. (A) Relative expression level of the genes of interest in all four patients together during all available manic and depressed phases. For comparability of expression shifts among patients, every subject's episodic shifts were normalized to his individual mean value $(100 \%)$. (B-E) Presentation of individual raw data (normalized to $G A P D H)$ for all patients separately. The n values given in brackets in (A)-(E) represent the total numbers of samples taken per affec- $\mathbf{2 5}$ tive state, in (A) referring to all four patients, and in (B)-(E) referring to individual patients. Mean \pm standard error is presented. 26 $A K R / C 3=$ aldo-ketoreductase family 1 , member $\mathrm{C} 3 ; P 2 R X 7$, purinergic receptor $\mathrm{P} 2 \mathrm{X}$, ligand-gated ion channel $7 ; P T G D S=$ pros- 27 taglandin D2 synthase; PTGS2 = prostaglandin-endoperoxide synthase 2 .

Figure 2A integrates the PBMC gene expression levels of all repeated measurements during manic and depressed episodes for all four rapid cycling patients. For this integrative approach, data obtained from each individual patient had to be first standardized to the individual mean expression level of each gene. This was done by calculating the mean of all measured values per gene regardless of episode, and setting it to $100 \%$. All individual data obtained were then expressed for

22 each gene as a percentage of this mean value. This allowed the integration of all patients with their repeated measurements into Figure 2A. The necessity for processing using this method

23 becomes obvious upon inspection of the variable 24 individual expression levels shown in Figures 2BE. The individual patient panels reveal a consistent trend of an upregulation of PTGDS mRNA during depressed phases as detected in our origi- nal case (3). In contrast, $A K R I C 3$ showed only an inconsistent trend of increased expression, which failed to become significant in the integrative approach (Fig. 2A). The new readout PTGS2/ $C O X 2_{P A N}$ revealed a trend of an increase in depressed episodes in three of the four subjects, resulting in borderline significance upon integration. The control gene, $P 2 R X 7$, did not show any steady trend in these four patients.

Figures $3 \mathrm{~A}-\mathrm{D}$ display the raw data (normalized to $G A P D H$ ) for PTGDS, AKRIC3, P2RX7 and $P T G S 2 / C O X 2_{\text {PAN }}$ mRNA expression in PBMC obtained from 43 patients with bipolar disorder in manic, depressed, hypomanic, and euthymic episodes, respectively (total $\mathrm{N}=162$ ). Analogously, 28 Figures 3E-H present data for 97 patients with monopolar depression in depressed and euthymic episodes (total $\mathrm{N}=237$ ). Obviously, there is no evidence of episode-specific expression changes of 


\section{Gurvich et al.}
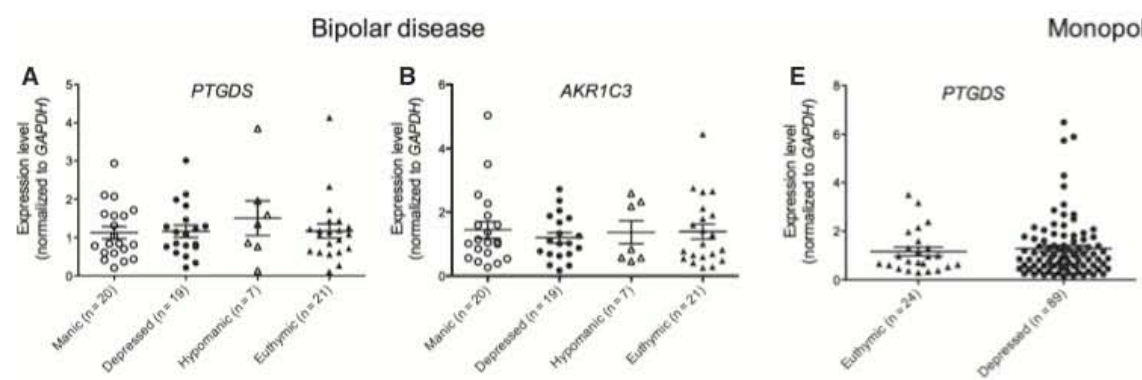

Monopolar depression
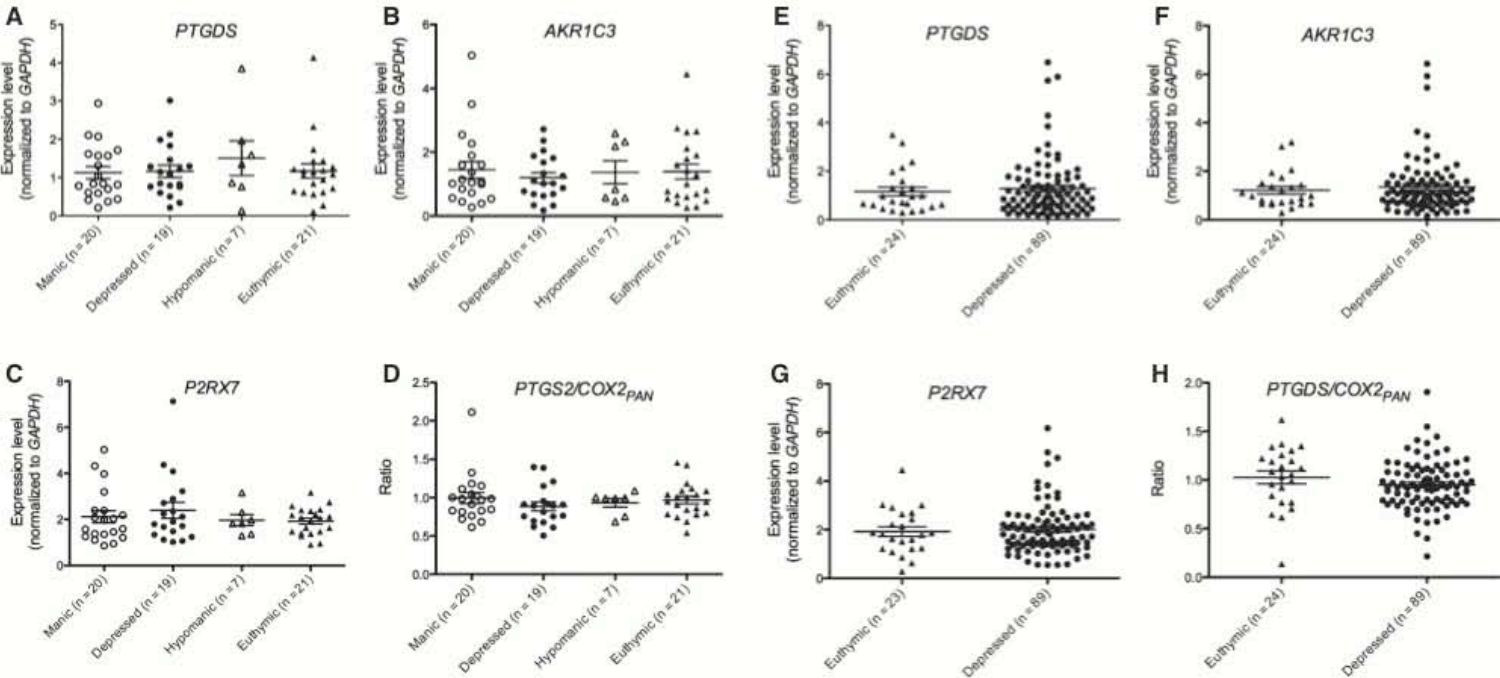

Fig. 3. (A-D) Scatter plots of episode-specific gene expression in 162 peripheral blood mononuclear cell (PBMC) samples from 43 patients with bipolar disorder. (E-H) Scatter plots of episode-specific gene expression in 237 PBMC samples from 97 patients with monopolar depression. Each symbol represents the mean value of all data obtained from each individual patient in the respective phase. Therefore, $n$ values do not add up to sample numbers. The differing numbers $(n=24$ versus $n=23)$ in euthymic phases shown in (E) and (G) are due to missing data for one patient sample with technical problems in the polymerase chain reaction. $A K R 1 C 3=$ aldo-ketoreductase family 1 , member $\mathrm{C} 3 ; P 2 R X 7$, purinergic receptor $\mathrm{P} 2 \mathrm{X}$, ligand-gated ion channel $7 ; P T G D S=$ pros- $\mathbf{2}$ taglandin D2 synthase; PTGS2 = prostaglandin-endoperoxide synthase 2.

these genes in the two groups of patients (Fig. 3). We note, however, that only a very limited number of samples per individual (one to four per subject on average) could be obtained over the recruitment period of three years, reflecting the much rarer occurrence of episodes in non-rapid cycling cases. This precluded an overall analysis of individual means in a fashion comparable to the rapid cycling subjects. The possibility thus cannot be excluded that interindividual mean expression variability rather than the restriction of a specific role for prostaglandin-related genes to rapid cycling accounts for the negative result.

\section{Discussion}

In the present study, the prostaglandin synthesisrelated gene expression findings of our previous case report on a patient with an extreme form of rapid cycling (3) could essentially be independently replicated in another four patients with this condition. The mRNA levels of PTGDS and PTGS2/ $C O X 2_{P A N}$ were significantly increased in pooled PBMC samples during depressed episodes. These findings, together with the observed alleviation of symptoms under COX2 inhibition in our case report, support an involvement of prostaglandins in rapid cycling. In contrast, in the present ana- lyzed PBMC samples from two larger cohorts of non-rapid cycling bipolar disorder and depressed patients, no episode-specific expression of these genes was detected.

We are fully aware that PBMC are not brain tissue (which would be impossible to obtain repeatedly during rapid cycling episodes in humans) and that our sometimes borderline significant findings could be epiphenomena rather than an indication of causality. Another limitation of the present study is the fact that we did not measure and quantify protein or enzymatic products and metabolites. For this, the required amount of material for reliable, repeated analyses would not easily be available and most likely be subject to even higher variability than already observed for mRNA expression. Nevertheless, the efficacy of prostaglandin-targeting treatment and its complete loss upon cessation thereof in the reported case may underscore at least some causal relationship of the mRNA expression changes measured here with the disease process.

Remarkably, recent clinical trials used COX2 inhibitors for treatment of affective disorders with the idea of applying an anti-inflammatory strategy to these diseases $(6,7,19-21)$. Even though our approach was originally derived from our pathological hibernation hypothesis (3), the effectiveness 
of these regimens lends further support to a causal role of the prostaglandin cascade in these illnesses.

According to the present findings, PTGDS as

31 the enzyme responsible for formation of $\mathrm{PGD}_{2}$

32 apparently plays a predominant role in rapid cycling. Notably, a recent report on attention-deficit hyperactivity disorder, which is even considered as a possible prodrome of bipolar disorder (22), described elevated expression of PTGDS in this condition (23).

33 In contrast to PTGDS, we were unable to reliably replicate the episode-specific expression pattern of $A K R I C 3$ observed in our previously reported case. PTGDS and $A K R I C 3$ are functionally closely related regarding the generation of

34,35 $\mathrm{PGF}_{2}$ metabolites from $\mathrm{PGD}_{2}$ (Fig. 1B). The consistently higher expression in depressed phases of

36 PTGDS but not $A K R I C 3$ may emphasize a central role of its product, $\mathrm{PGD}_{2}$, in mood and sleep regulation (24-28), with also the latter heavily affected

37 in bipolar disorders (29). A strong stimulation or accumulation of $\mathrm{PGD}_{2}$ in the course of a severe rapid cycling syndrome may lead to a more pronounced induction of $A K R I C 3$ (30). This could explain the increase in $A K R I C 3$ in the original case

38 (3) and would also be consistent with the similar tendency observed in patient 3 with his particularly

39 accentuated psychopathology (Table 1).

Although the involvement of prostaglandins in rapid cycling is further supported by this study, no conclusions can be drawn at this point regarding non-rapid cycling bipolar disorder or monopolar depression. This may be due to the limited number of blood samples per individual obtained within the relatively short recruitment and follow-up period of $\leq 3$ years. High interindividual variation in PBMC-derived mRNA expression levels and the small number of samples per individual (precluding a calculation of individual mean expression values as performed here for rapid cycling patients) may have veiled a potential regulation of prostaglandin-associated genes. An alternative explanation could be that rapid cycling constitutes a biologically separate subgroup of bipolar disease involving prostaglandins and resembling features of the hibernation program $(4,5,31,32)$. Prospective studies on more subjects and with longer individual follow-up with more PBMC samples per person would possibly help to clarify this issue. The beneficial effects of the use of COX 2 inhibitors

40 as an anti-inflammatory strategy obtained in recent clinical trials for affective disorders $(6,7,19-21)$, however, may already indicate a broader role of prostaglandins in these conditions.

Unfortunately, there is no construct-valid animal model known for bipolar disease, which would be helpful to gain mechanistic insights into the dramatic and disabling intraindividual behavioral shifts in humans. We envision that transgenic mice with inducible and reversible $P T G D S$ expression in the brain might be useful as a first step to model and study episode-specific gene expression changes and their behavioral consequences.

Interestingly, in a recent genome-wide association study in bipolar disease, a significant association signal [single nucleotide polymorphism (SNP) 41 rs4650608] was found in close proximity to PTGFR 42 (33). This lends further support to the hypothesis that prostaglandins are causally involved in the pathogenesis of bipolar disorder. The low odds ratio (1.14 for the $\mathrm{T}$ allele) would support the notion that only a subgroup of individuals may be characterized by a genetic predisposition involving the prostaglandin pathway.

To conclude, the present data indicate once more a potential causal relationship of the prostaglandin system with rapid cycling bipolar disorder. 43 Based on these findings, the development of novel treatment strategies for rapid cycling and of adequate animal models to study the pathogenesis of this severe disorder should be encouraged.

\section{Acknowledgements}

This work was supported by the Max Planck Society, the Max Planck Förderstiftung, and the DFG (CNMPB).

\section{Disclosures}

The authors of this paper do not have any commercial associations that might pose a conflict of interest in connection with this manuscript.

\section{Author contributions}

$\mathrm{MB}$, with the help of $\mathrm{AG}$, performed all patient-related work, i.e., recruitment, psychopathology rating, and blood sampling. AG, supported by LD, conducted all lab work and qPCR data analysis. AG and HE performed the statistical analyses and designed the figures. HE, supported by MB, DS, and KM, planned, supervised, and coordinated the project, and interpreted the final data. $\mathrm{HE}, \mathrm{MB}$, and AG wrote the manuscript. All authors contributed to all versions of this paper.

\section{References}

1. Kupka RW, Luckenbaugh DA, Post RM, Leverich GS, Nolen WA. Rapid and non-rapid cycling bipolar disorder: a meta-analysis of clinical studies. J Clin Psychiatry 2003; 64: 1483-1494

2. Diagnostic and Statistical Manual of Mental Disorders: DSM-5, 5th edn. Washington, DC: American Psychiatric Association, 2013.

3. Begemann M, Sargin D, Rossner MJ et al. Episode-specific differential gene expression of peripheral blood mono- 


\section{Gurvich et al.}

nuclear cells in rapid cycling supports novel treatment approaches. Mol Med 2008; 14: 546-552.

4. Takahata R, Matsumura H, Eguchi $\mathrm{N}$ et al. Seasonal variation in levels of prostaglandins D2, E2 and F2(alpha) in the brain of a mammalian hibernator, the Asian chipmunk. Prostaglandins Leukot Essent Fatty Acids 1996; 54: 77-81.

5. O'Hara BF, Watson FL, Srere HK et al. Gene expression in the brain across the hibernation cycle. J Neurosci 1999; 19: $3781-3790$

6. Muller N, Schwarz MJ, Dehning S et al. The cyclooxygenase-2 inhibitor celecoxib has therapeutic effects in major depression: results of a double-blind, randomized, placebo controlled, add-on pilot study to reboxetine. Mol Psychiatry 2006; 11: 680-684.

7. Akhondzadeh S, Jafari S, Raisi F et al. Clinical trial of adjunctive celecoxib treatment in patients with major depression: a double blind and placebo controlled trial. Depress Anxiety 2009; 26: 607-611.

8. Hamilton M. Development of a rating scale for primary depressive illness. Br J Soc Clin Psychol 1967; 6: 278-296.

9. Beck AT, Ward CH, Mendelson M, Mock J, Erbaugh J. An inventory for measuring depression. Arch Gen Psychiatry 1961; 4: 561-571.

10. Young RC, Biggs JT, Ziegler VE, Meyer DA. A rating scale for mania: reliability, validity and sensitivity. $\mathrm{Br} \mathrm{J}$ Psychiatry 1978; 133: 429-435.

11. Kay SR, Fiszbein A, Opler LA. The Positive and Negative Syndrome Scale (PANSS) for schizophrenia. Schizophr Bull 1987; 13: 261-276.

12. Censarek P, Freidel K, Udelhoven $M$ et al. Cyclooxygenase COX-2a, a novel COX-2 mRNA variant, in platelets from patients after coronary artery bypass grafting. Thromb Haemost 2004: 92: 925-928.

13. Censarek P, Steger G, Paolini C et al. Alternative splicing of platelet cyclooxygenase- 2 mRNA in patients after coronary artery bypass grafting. Thromb Haemost 2007; 98 1309-1315.

14. Pasinetti GM, Aisen PS. Cyclooxygenase-2 expression is increased in frontal cortex of Alzheimer's disease brain. Neuroscience 1998; 87: 319-324.

15. Chang JW, Coleman PD, O'Banion MK. Prostaglandin $\mathrm{G} / \mathrm{H}$ synthase-2 (cyclooxygenase-2) mRNA expression is decreased in Alzheimer's disease. Neurobiol Aging 1996; 17: 801-808.

16. Backlund L, Lavebratt C, Frisen L et al. P2RX7: expression responds to sleep deprivation and associates with rapid cycling in bipolar disorder type 1. PLoS ONE 2012; 7: e43057.

17. Barden N, Harvey M, Gagne B et al. Analysis of single nucleotide polymorphisms in genes in the chromosome 12Q24.31 region points to $\mathrm{P} 2 \mathrm{RX} 7$ as a susceptibility gene to bipolar affective disorder. Am J Med Genet B Neuropsychiatr Genet 2006; 141B: 374-382.

18. Feng WP, Zhang B, Li W, Liu J. Lack of association of P2RX7 gene rs2230912 polymorphism with mood disorders: a meta-analysis. PLoS ONE 2014; 9: e88575.

19. Muller N. COX-2 inhibitors as antidepressants and antipsychotics: clinical evidence. Curr Opin Investig Drugs 2010; 11: 31-42.

20. Fond G, Hamdani N, Kapczinski F et al. Effectiveness and tolerance of anti-inflammatory drugs' add-on therapy in major mental disorders: a systematic qualitative review. Acta Psychiatr Scand 2014; 129: 163-179.
21. Leboyer M, Soreca I, Scott J et al. Can bipolar disorder be viewed as a multi-system inflammatory disease? J Affect Disord 2012; 141: 1-10.

22. Singh MK, DelBello MP, Kowatch RA, Strakowski SM. Co-occurrence of bipolar and attention-deficit hyperactivity disorders in children. Bipolar Disord 2006; 8: 710-720.

23. Marin-Mendez JJ, Patino-Garcia A, Segura V, Ortuno F, Galvez MD, Soutullo CA. Differential expression of prostaglandin D2 synthase (PTGDS) in patients with attention deficit-hyperactivity disorder and bipolar disorder. J Affect Disord 2012; 138: 479-484.

24. Onoe H, Ueno R, Fujita I, Nishino H, Oomura Y, Hayaishi O. Prostaglandin D2, a cerebral sleep-inducing substance in monkeys. Proc Natl Acad Sci USA 1988; 85; 4082-4086.

25. Hayaishi O. Prostaglandin D2 and sleep-a molecular genetic approach. J Sleep Res 1999; 8 (Suppl. 1): 60-64.

26. Matsumura H, Nakajima T, Osaka T et al. Prostaglandin D2-sensitive, sleep-promoting zone defined in the ventral surface of the rostral basal forebrain. Proc Natl Acad Sci USA 1994; 91: 11998-12002.

27. Urade Y, Hayaishi O. Prostaglandin D2 and sleep/wake regulation. Sleep Med Rev 2011; 15: 411-418.

28. Haack M, Lee E, Cohen DA, Mullington JM. Activation of the prostaglandin system in response to sleep loss in healthy humans: potential mediator of increased spontaneous pain. Pain 2009; 145: 136-141.

29. Plante DT, Winkelman JW. Sleep disturbance in bipolar disorder: therapeutic implications. Am J Psychiatry 2008; 165: 830-843.

30. Mantel A, Carpenter-Mendini AB, Vanbuskirk JB, De Benedetto A, Beck LA, Pentland AP. Aldo-keto reductase $1 \mathrm{C} 3$ is expressed in differentiated human epidermis, affects keratinocyte differentiation, and is upregulated in atopic dermatitis. J Invest Dermatol 2012; 132: 1103-1110.

31. Prendergast BJ, Freeman DA, Zucker I, Nelson RJ. Periodic arousal from hibernation is necessary for initiation of immune responses in ground squirrels. Am J Physiol Regul Integr Comp Physiol 2002; 282: R1054-R1062.

32. Donahue SW, Galley SA, Vaughan MR et al. Parathyroid hormone may maintain bone formation in hibernating black bears (Ursus americanus) to prevent disuse osteoporosis. J Exp Biol 2006; 209: 1630-1638.

33. Chen DT, Jiang X, Akula N et al. Genome-wide association study meta-analysis of European and Asian-ancestry samples identifies three novel loci associated with bipolar disorder. Mol Psychiatry 2013; 18: 195-205.

34. Vane JR, Bakhle YS, Botting RM. Cyclooxygenases 1 and 2. Annu Rev Pharmacol Toxicol 1998; 38: 97-120.

35. FitzGerald GA. COX-2 and beyond: approaches to prostaglandin inhibition in human disease. Nat Rev Drug Discov $2003 ; 2: 879-890$.

36. Matsuura K, Shiraishi H, Hara A et al. Identification of a principal mRNA species for human 3alpha-hydroxysteroid dehydrogenase isoform (AKR1C3) that exhibits high prostaglandin D2 11-ketoreductase activity. J Biochem 1998; 124: $940-946$.

37. Costine BA, Inskeep EK, Blemings KP, Flores JA, Wilson ME. Mechanisms of reduced luteal sensitivity to prostaglandin F2alpha during maternal recognition of pregnancy in ewes. Domest Anim Endocrinol 2007; 32: 106-121. 


\subsection{Original manuscript: Drosophila project}

Research report

\section{Monogenic heritable autism gene neuroligin impacts Drosophila social behaviour}

Nina Hahn ${ }^{a}$, Bart Geurten ${ }^{\mathrm{a}, \mathrm{e}}$, Artem Gurvich ${ }^{\mathrm{b}, \mathrm{c}}$, David Piepenbrock ${ }^{\mathrm{a}, \mathrm{e}}$, Anne Kästner ${ }^{\mathrm{b}, \mathrm{c}}$, Damiano Zanini ${ }^{a}$, Guanglin Xing ${ }^{d}$. Wei Xie ${ }^{d}$, Martin C. Göpfert ${ }^{\mathrm{a}, c, e}$, Hannelore Ehrenreich ${ }^{\mathrm{b}, \mathrm{c}}$, Ralf Heinrich ${ }^{\mathrm{a}, *}$

a Department of Cellular Neurobiology, Institute for Zoology, University of Göttingen, 37077 Göttingen, Germany

${ }^{\mathrm{b}}$ Clinical Neuroscience, Max Planck Institute for Experimental Medicine, 37077 Göttingen, Germany

'DFG Research Center for Nanoscale Microscopy and Molecular Physiology of the Brain, Germany

${ }^{d}$ The Key Laboratory of Developmental Genes and Human Diseases, Institute of Life Sciences, Southeast University, Nanjing 210009, China

- DFG Collaborative research Center SFB 888-C2, Germany

\section{H I G H L I G H T S}

- Social behaviour of neuroligin2deficient Drosophila melanogaster was studied.

- Social interactions are reduced and acoustic communication signals altered.

- Mutant flies have normal general activity and intact sensory perception.

- Neuroligin2-deficiency affects brain circuits that coordinate social behaviours.

- Mutant flies display the behavioural core symptoms of autism spectrum disorders.

\section{A R T I CLE I N F O}

Article history:

Received 4 January 2013

Received in revised form 31 May 2013

Accepted 13 June 2013

Available online 19 June 2013

Keywords:

Drosophila melanogaster

Neuroligin

Social behaviour

Acoustic communication

Behavioural transition

Autism
G R A P H I C A L A B S T R A C T
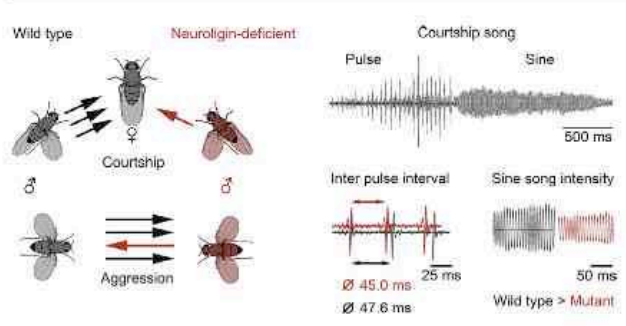

A B S T R A C T

Autism spectrum disorders (ASDs) are characterized by deficits in social interactions, language development and repetitive behaviours. Multiple genes involved in the formation, specification and maintenance of synapses have been identified as risk factors for ASDs development. Among these are the neuroligin genes which code for postsynaptic cell adhesion molecules that induce the formation of presynapses, promote their maturation and modulate synaptic functions in both vertebrates and invertebrates. Neuroligin-deficient mice display abnormal social and vocal behaviours that resemble ASDs symptoms. Here we show for the fly Drosophila melanogaster that deletion of the $d n / 2$ gene, coding for one of four Neuroligin isoforms, impairs social interactions, alters acoustic communication signals, and affects the transition between different behaviours. dnl2-Deficient flies maintain larger distances to conspecifics and males perform less female-directed courtship and male-directed aggressive behaviours while the patterns of these behaviours and general locomotor activity were not different from wild type controls. Since tests for olfactory, visual and auditory perception revealed no sensory impairments of $d n l 2$-deficient mutants, reduced social interactions seem to result from altered excitability in central nervous neuropils that initiate social behaviours. Our results demonstrate that Neuroligins are phylogenetically conserved not only regarding their structure and direct function at the synapse but also concerning a shared implication in the regulation of social behaviours that dates back to common ancestors of humans and flies.

\footnotetext{
* Corresponding author at: Department of Cellular Neurobiology, Schwann-Schleiden-Research Center, Julia-Lermontowa-Weg 3, 37077 Göttingen, Germany.

Tel.: +49551 39177958; fax: +4955139177952.

E-mail address: rheinri1@gwdg.de (R. Heinrich).
} 
In addition to previously described mouse models, Drosophila can thus be used to study the contribution of Neuroligins to synaptic function, social interactions and their implication in ASDs.

(C) 2013 Published by Elsevier B.V.

\section{Introduction}

Neuroligins represent a family of postsynaptic cell adhesion molecules that establish bidirectional trans-synaptic signalling complexes via association with presynaptic neurexins [1-3]. Neuroligins' intracellular domains are important for the accumulation of postsynaptic density proteins (including PSD-95, SAPAP, Homer and subunits of metabotropic and ionotropic glutamate receptors at excitatory synapses and collybistin, S-SCAM and GABA $A^{-}$ or glycine receptors at inhibitory synapses) through direct association with Shank or gephyrin scaffolding proteins [3-5]. Neuroligins' extracellular domains bind presynaptic Neurexins which serve as organizers for presynaptic scaffolding proteins and proteins of the synaptic release machinery [6]. Different genes and a number of splice variants of these trans-synaptic adhesion proteins have been suggested to contribute to a combinatorial code that determines synapse identity and network connectivity during brain development [3]. Impairments of several components of this transsynaptic signalling complex, including Neuroligins, Neurexins, and Shanks, have been associated with autism spectrum disorders (ASDs) $[1,2,4,5,7]$ that are characterized by three core behavioural symptoms: impairment in language and communication, deficits in social interactions and stereotyped repetitive actions.

Though Neuroligins can induce the formation of presynapses they are not required for synaptogenesis but rather promote the maturation, maintenance and activity dependent modulation of synapses [8-10]. In humans, especially neuroligin 3 and neuroligin 4 have been linked to autistic phenotypes [11] and mice deficient of these or other neuroligin genes have impaired synaptic release $[8,9]$ and plasticity $[10]$ and display abnormal social and vocal behaviours [12-15]. Deficits in social behaviour of mice and humans are suggested to arise from unbalanced excitatory and inhibitory synaptic signalling in relevant brain circuits [16] caused by inactivation of particular Neuroligin isoforms that preferably function at excitatory or inhibitory synapses $[8,17,18]$.

Neuroligins and Neuroligin-Neurexin trans-synaptic interactions are conserved from nematodes, molluscs, and insects to mammals [19]. Insects, like mammals, contain multiple neuroligin genes that lead to a variety of protein isoforms by alternative splicing [20]. Phylogenetic analyses of neuroligin gene sequences revealed distinct clusters of vertebrate, insect and nematode homologs that emerged from a single common ancestor in sea urchins [20]. Drosophila contains four neuroligin genes (dnl14) none of which displaying a particular similarity with one of the human orthologs. Only two of these four neuroligins in Drosophila melanogaster, dnl1 specifically expressed in muscle and dnl2 expressed in muscle and neuronal postsynapses, have so far been studied for their role in the formation and function of neuromuscular junctions (NMI). Absence of either Dnl1 or Dnl2 does not prevent NMJ formation but interferes with NMJ expansion, active zone addition, and the molecular composition of glutamate receptors during subsequent muscle development causing an overall reduced (dnl1 deletion) or increased (dnl2 deletion) synaptic excitation in the mature neuromuscular system $[21,22]$.

A large number of human disease genes have well conserved homologues in the Drosophila genome [23] and Drosophila is increasingly used to study mechanisms of neurodegenerative and neurological diseases (e.g. Parkinson's Disease; Alzheimer's Disease, fragile $\times$ syndrome) while its potential value as a model system for disorders that are mainly diagnosed by psychiatric phenotypes has not yet been recognized (reviewed in [24]).
Given the association of human Neuroligins with ASDs and assuming that social behaviours are regulated by central nervous circuits (which excludes a role of $d n l 1$ being exclusively expressed at the neuromuscular junction) we analyzed acoustic communication signals and social behaviour of a Drosophila dnl2 null mutant ( $d n / 2^{K O 17}$ generated and characterized for its function at the NM] by Sun et al. [22]). Our studies revealed characteristic alterations in social interactions and communication patterns that resemble autism-like phenotypes described in ASD patients and mouse models for this disease. Since control experiments revealed no impairments of motor coordination, general activity and peripheral sensory processing, the observed behavioural phenotypes of dnl2-deficient flies must result from altered excitability in central nervous circuits that initiate and coordinate social and other behaviours in Drosophila.

\section{Materials and methods}

\subsection{Animals}

Studies were performed with wild type Cantons Drosophila melanogaster and a dn/2-deficient mutant line $\left(d n / 2^{\mathrm{KO} 17}\right)$, generated by targeted knockout of the $d n / 2$ genomic locus [22]. The mutants had been outcrossed into Canton $S$ background through several generations. Virgin 7 day old decapitated wild type females were used as courtship targets. Behavioural tests were performed with 7-12 days old socially naive males, that have been isolated for individual housing within two hours after eclosion. All recordings were made at room temperature $\left(20-22^{\circ} \mathrm{C}\right)$.

Some experiments were repeated with another dni2-deficient mutant line Some experiments were repeated with another dni2-deficient mutant line
$\left(d n 2^{K 070}\right)[22]$, showing similar though in most cases weaker effects on acoustic communication signals and social interactions. Similar studies were also attempted with neurexin-deficient $D$. melanogaster (dnrx 1 described in [25]). Since these flies had severe locomotor impairments and were not able to produce any acoustic communication signals, there detailed behavioural analysis was not completed.

\subsection{Sound recordings}

Male courtship songs were recorded with a microphone (Bruel \& Kjaer Type $4165)$ in a soundproof chamber, amplified, band pass filtered $(70-5.000 \mathrm{~Hz})$ and directly digitized with a sampling frequency of $44.100 \mathrm{~Hz}$. The software Audacity 1.3.12beta (http://audacity.sourceforge.net) was used for acoustic data acquisition and analysis. Frequency spectra were determined by Fast Fourier Transformation with a 4096 width Hanning window. Maximal song intensities Transformation with a 4096 width Hanning window. Maximal song intensities
were compared on a relative basis from recordings with identical settings, registered as dBFS (logarithmic scale without absolute calibration) by the Audacity software. Statistical comparison of song parameters (usually not normally distributed but with equal variance) was performed with the Mann-Whitney rank sum test.

\section{Inter individual distance assay}

Ten mature flies ( 5 males and 5 females) of same genotype were simultaneously aspirated into an equally illuminated featureless round arena $(665 \mathrm{~mm}$ ). Flies were allowed to disperse and explore the arena for $5 \mathrm{~min}$. Subsequently, their individual positions were recorded (at $100 \mathrm{~Hz}$ frame rate with a Dalsa GigaE (Dalsa) camera) during five periods of 1 min duration, starting at 5, 8, 11, 14 and $17 \mathrm{~min}$ after introduction to the arena. Three experiments were performed with both wild type and $d n 12^{\mathrm{KO} I 7}$ mutant flies giving data for 30 flies per genotype. Flies' positions and orientations were tracked with the ivTools software suite (developed by Lindemann \& Braun, https://opensource.cit-ec.de/projects/ivtools). MatLab (Mathworks Inc.) was used to determine the median distance of each fly to all other flies in the assay on 60.000 frames per experiment and to calculate its median distance to the other flies over the entire observation period. Durations of males' unilateral wing extensions were determined from the recorded movies by an observer that was blind to the genotype of the flies. For statistic mutation tests on the differences between the medians of different experimental groups were performed. Permutation tests belong to the family of exact significance test as first introduced by Fisher $[26]$ and refined by various authors [e.g. $27,28]$. 


\subsection{Competitive courtship assay}

One day before behavioural testing male flies were labelled by small dots of acrylic paint on the dorsal thorax. Behavioural assay was performed with one decapitated virgin female and two males that were simultaneously aspirated into a round arena ( $(4 \mathrm{~mm})$. After $5 \mathrm{~min}$ acclimatization, behaviour was recorded from above with a webcam (Hercules, Deluxe Optical Glass) with a frame rate of $30 \mathrm{~Hz}$ during observation periods of $15 \mathrm{~min}$ and movies were stored in avi format. Courtship and agonistic interactions during the $1 \mathrm{st}, 4 \mathrm{th}, 7 \mathrm{th}, 10 \mathrm{th}$ and 13 th minute were scored through analysis of individual frames $(2 \mathrm{~Hz}$ temporal resolution) by an observer that was blind to the genotype of the flies. Statistical analysis was identical to evaluation of data from inter individual distance assays. Since the same data set was used for comparison more than one time, we corrected the p-values with the Benjamini-Hochberg procedure [29,30]. For calculations we used the Matlab implementation of Benjamini and Hochberg's procedure by David M. Groppe, Dept. of Cognitive Science, University of California, San Diego (http://www. mathworks,com/matlabcentral/fileexchange/29274-mass-univariate-erp-toolbox/ content/fdr bh.m).

\subsection{Circadian locomotion assay}

Flies were placed in glass tubes ( $(3 \mathrm{~mm}$; length $7 \mathrm{~mm}$ ) sealed with food on one end and with a gas permeable cap on the other. The glass tubes were introduced into a special incubator for assessment of circadian activity (Tritech Research, CircKinetics). Midline crossings of individual flies were automatically counted for $96 \mathrm{~h}$ ( 4 days with $12 \cdot 12 \mathrm{~h}$ light dark cycles at $24^{\circ} \mathrm{C}$ ) starting with the first complete light phase $>15 \mathrm{~h}$ after introduction to the tubes. Numbers of flies' centre crossings were no normally distributed and therefore evaluated by ANOVA on ranks (Kruskal-Wallis) and post hoc Mann-Whitney rank sum test.

\subsection{Electroretinogram}

Flies were waxed to a teflon holder and placed in a dark box. Light stimuli were generated with a 3 W white InGaN-LED (model ASMT-MWE2-NMNO0, Avago Technologies). Stimuli were delivered through a glass optical fibre $(\varphi 0.5 \mathrm{~mm})$ contained in a hypodermic needle whose opening was positioned perpendicular to the eye's surface at a distance of $0.5 \mathrm{~mm}$. The hypodermic needle was connected with the eye via a drop of conductive gel (SignaGel, Parker Laboratories) to serve as recording electrode. A second sharp hypodermic needle was impaled below the fly's scutellum as indifferent electrode. Recorded signals were amplified with a custom built high input-impedance DC amplifier, A/D converted with a sampling rate of $20 \mathrm{kHz}$ with a Micro1401-3 data acquisition box (Cambridge Electronic Design) and registered with Spike 2 (version 7.09, Cambridge Electronic Design).

\subsection{Auditory functions}

Peripheral auditory functions were tested as described in [31]. Vibrations of the flies' sound receiver, the arista, were measured by laser Doppler vibrometry (PSV 400, Polytec $\mathrm{GmbH}$ ). Best frequencies of arista oscillations were determined from the power spectrum of its vibrations in the absence of sound stimulation. Antennae were stimulated with pure tones at their individual best frequency and with artificial pulse song (inter pulse interval $30 \mathrm{~ms}$, carrier frequency $170 \mathrm{~Hz}$ ), Stimulus particle velocity at the fly's position in the sound field was measured with a pressure gradient microphone (Emkay NR 3158, Knowles Electronics Inc.). Compound action potentials (CAPs) were recorded with an electrolytically tapered tungsten electrode inserted between antenna and head and the indifferent electrode place in the scutellum. Recorded signals during stimulation with different sound intensities were digitized with $12.1 \mathrm{kHz}$ sampling rates and subjected to Fast Fourie Transformation. CAP amplitudes were determined as Fourier amplitudes at twice the stimulus frequency.

\section{Results}

\subsection{Acoustic communication signals}

Courtship songs of $d n / 2$-deficient males contained regular pulse and sine songs (Fig. 1A) as typically produced by wild type flies during courtship [32]. The average inter pulse interval, a temporal parameter critical for species recognition and song attractiveness [33], was significantly shorter in pulse songs of $d n l 2^{K 017}$ mutants $(45 \mathrm{~ms})$ than in wild type males ( $47.6 \mathrm{~ms} ; p=0.009$ ) (Fig. 1B). In contrast, regularity and numbers of pulses within pulse trains, sound intensity of pulses, numbers of oscillations per pulse, and average intra pulse frequencies (wild type: $214.6 \mathrm{~Hz}$; $d n l 2^{\mathrm{K} 017}$ : $230.7 \mathrm{~Hz}$ ) were not different between genotypes. Sine songs of dn $2^{\mathrm{KO}}{ }^{27}$ mutants were significantly reduced in volume compared

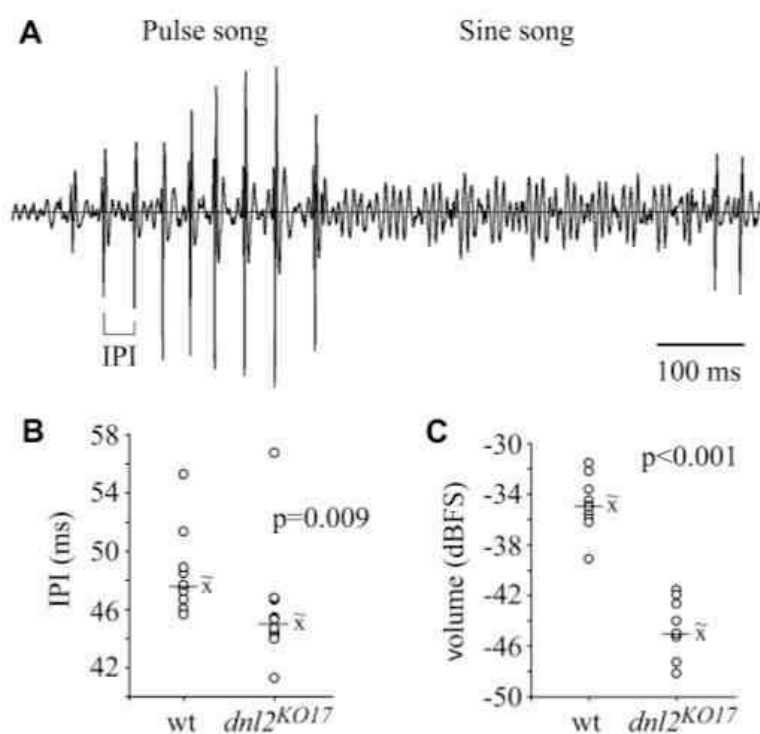

Fig. 1. Acoustic communication signals of male dnl2-deficient and wild type $D$. melanogaster. (A) Oscillogram of a courtship song with typical pulse song and sine song patterns generated by a dnl2 ${ }^{\mathrm{KO} 17}$ mutant male. Inter pulse interval (IPI) is indicated. (B) dnI2 ${ }^{\mathrm{KO} 17}$ mutant males have shorter IPI duration than wild type flies (wt). $N=10$ for each genotype, each value represents the average of IPIs from three randomly chosen pulse trains with at least 14 pulses. (C) Sine song intensity is reduced in dnl2 $2^{\mathrm{KOIZ}}$ mutant males compared to wild type males. $n=10$ for each genotype, each value represents the average of the maximal sound intensities from three randomly chosen sine songs.

to those of wild type males ( $p<0.001$ ) (Fig. 1C) but did not differ in any other characteristic including the average sine song frequency (wild type: $125 \mathrm{~Hz}$; dnl2 ${ }^{\mathrm{KO} 17}: 121.8 \mathrm{~Hz}$ ) and the duration of sine song sequences. Thus, courtship songs of $d n l 2^{K 017}$ mutants were similar to those of wild type D. melanogaster in all except two parameters, the duration of inter pulse intervals and the intensity of sine songs.

\subsection{Inter individual distance and transition between behaviours}

To study the distribution and behaviour of small fly groups, we placed five male with five female flies of the same genotype into a featureless round arena. The median distance of individual flies to all other flies in the arena was significantly larger in $d n l 2^{K O 17}$ mutants (median $34.94 \mathrm{~mm}$ ) than in wild type flies (median $30.09 \mathrm{~mm}$ ) (Fig. 2A). While the median average distance between wild type flies gradually increased over the 5 observation periods of 1 min duration (from 26.12 to $34.25 \mathrm{~mm}$ ), dnl2 $2^{\mathrm{KO} 17}$ mutants maintained large inter individual distances already in the first observation period (median average distance: $38.11 \mathrm{~mm}$ ) with no regular increase in subsequent periods (Fig. 2B). This resulted in a gradual decrease of differences between wild type and mutant flies' median average distances with assay duration (from $p=0.0005$ in minute $5-6$ to no difference in minute $17-18$ ). In contrast, typical male to female distances during courtship where not different between wild type and $d n l 2^{K O 17}$ flies. If courted females moved away, wild type males immediately retracted the wing that produced the courtship song (median until wing retraction: $9.85 \mathrm{~s}$ ). dnl2-deficient males adjusted their behaviour to the new situation after longer latencies (median: $42.19 \mathrm{~s}$ ) and spent much longer periods with one extended wing than wild type males (Fig. 2C). A similar defect in timely switching from one behaviour to the next was concerned with locomotor patterns. While wild type flies change walking directions by stopping, turning into the new 
A

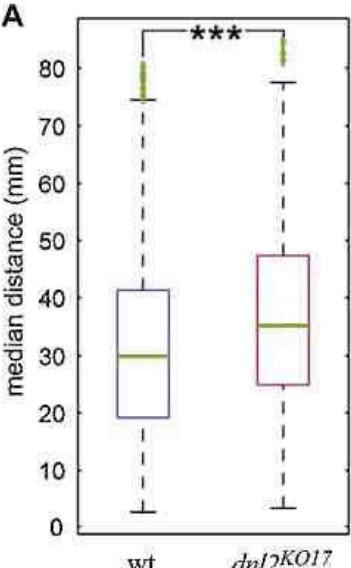

C

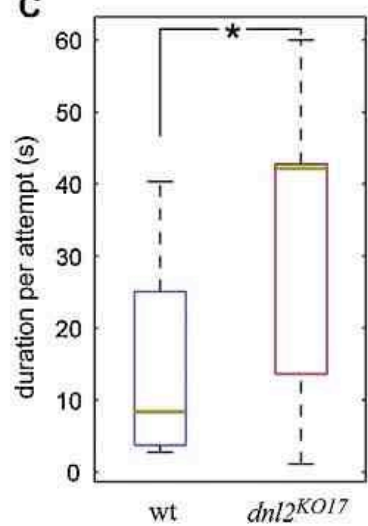

B

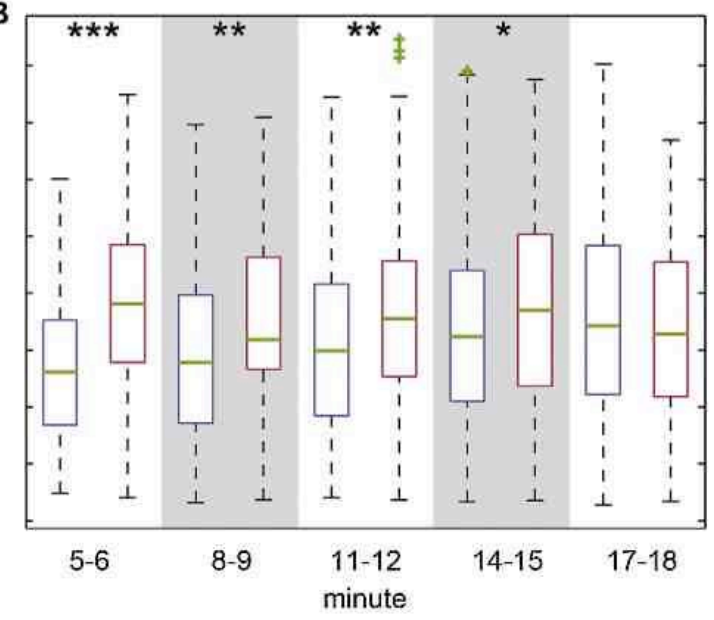

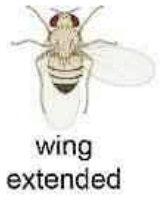

wing

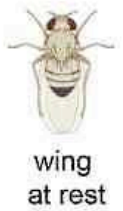

Fig. 2. Group behaviour of wild type and $d n i 2$-deficient $D$. melanogaster. (A) dnl2kol7 mutant flies maintain a larger median distance to nine conspecifics in a featureless arena than wild type flies (wt). dnl $2^{\mathrm{KOI}}$ : median $34.94 \mathrm{~mm}$, wt: median $30.09 \mathrm{~mm}, p=0.00001, \mathrm{~N}=30$ flies per genotype assayed in three trials with 10 flies; total of 5 periods of 1 min duration. (B) Average inter individual distances during the 5 individual periods analyzed: 5-6, 8-9, 11-12, 14-15 and $17-18$ min after introduction into the arena. Differences of average distance to conspecifics between wild type and mutant flies are large during the first period $(5-6: p=0.0005)$ and gradually decrease until the 5 th observation period ( $8-9 ; p=0.0029 ; 11-12 ; p=0.0147 ; 14-15 ; p=0.0367 ; 17-18$; no difference) due to a steady increase of inter individual distances between wild type flies. (C) Unilateral wing extension by wild type males was restricted to short periods of courtship in the immediate vicinity of a female. Instead of immediately retracting the extended wing when the courted female moved away, dn $22^{\mathrm{k} 017}$ mutant males usually did not terminate courtship posture but kept the wing in the extended position for significantly prolonged periods of time. dnl2 $2^{\text {k017 }}$; median 42.19 s, wt: median $9.85 \mathrm{~s}, p=0.0242$ ). "Represents $p<0.05$, " represents $p<0.01$, "*'represents $p<0.001$, permutation tests on median differences.

direction and continue walking, dnl2 ${ }^{K O 17}$ mutants do not separate translational and rotational activity but instead continue walking into the rotational phase and basically walk in a curve.

\subsection{Courtship and agonistic behaviour}

In the competitive courtship assay two males switch between male-male agonistic interactions and courtship towards one decapitated mature virgin wild type female. Either two socially naive males of the same genotype (equal assay with two wild type or two $d n l 2^{K O 17}$ ) or one male of each genotype (mixed assay) were combined in this assay and the time that each male spent performing male-directed agonistic behaviour and female-directed courtship behaviour was determined. In assays with males of equal genotype, $d n l 2^{K O 17}$ mutant males spent similar total durations courting the female, initiated courtship at similar frequencies but performed courtship bouts with prolonged duration, compared with wild type males (Fig. $3 \mathrm{~A}-\mathrm{C}$ ). In mixed assays, where one $d n l 2^{K O 17}$ mutant male directly competed with one wild type male, the mutants performed less courtship, initiated courtship more rarely and continued individual courtship bouts for shorter periods than wild type males (Fig. $3 \mathrm{~A}-\mathrm{C}$ ). In assays with two males of different genotype a large proportion (six out of ten) of $d n / 2$ deficient males performed no courtship at all while courtship activity of co-assayed wild type males was higher in most individual pairings but variable between different experiments (Fig. 3A). Total durations of agonistic interactions were reduced in $d n 2^{K O 17}$ mutant compared to wild type in both equal genotype and mixed genotype assays (Fig. 3D). The reduced total duration of agonistic interactions in $d n l 2^{K O 17}$ deficient males resulted from a lower tendency (not significant) to initiate respective behaviours in equal genotype assays (Fig. 3E) and from a shorter duration of individual agonistic bouts in mixed genotype competitions (Fig. 3F). Male fly agonistic encounters are terminated by retraction of the loser which often stimulates chasing by the winner [34] and the duration for which a fly continues an encounter has been associated with its aggressive motivation [35]. Our results therefore suggest a reduced motivation of dnl2-deficient males to engage in both male-directed agonistic interactions and female-directed courtship. 
A

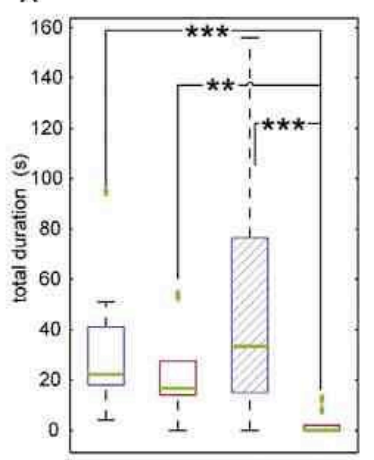

D

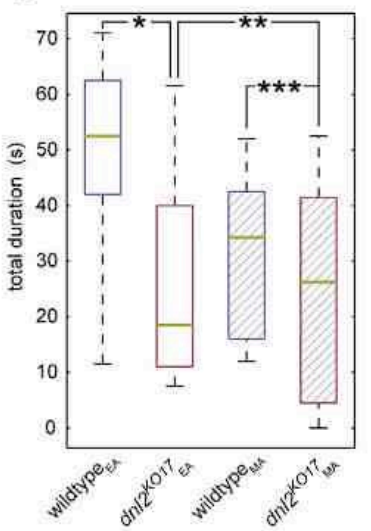

B

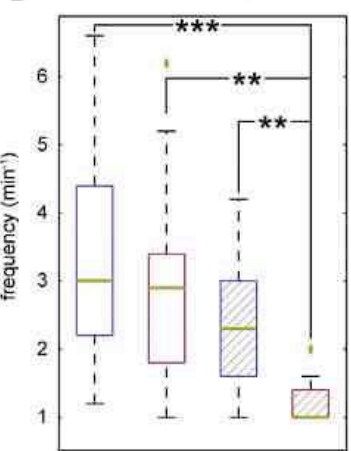

E

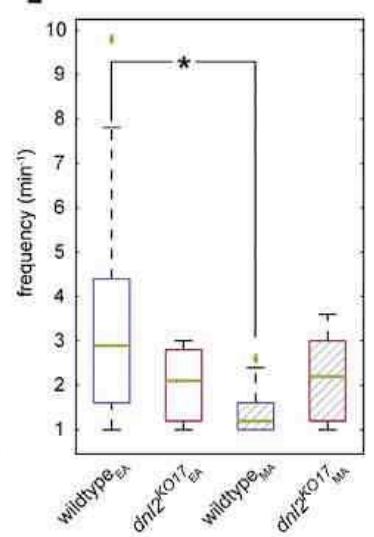

C

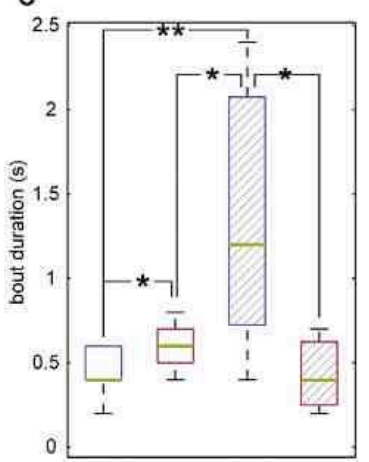

$\mathrm{F}$

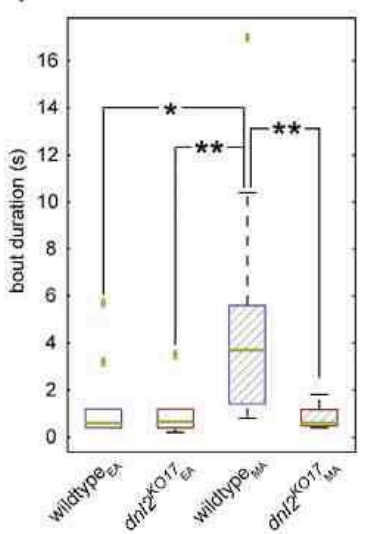

Fig. 3. Competitive courtship assays with wild type and dnl2-deficient $D$. melanogaster. (A) In assays with males of equal genotype (EA, first and second column) dni2 ${ }^{\mathrm{KO} 17}$ mutant males spent slightly less time (not significant) courting the female than wild type (wt) males. When males of both genotypes directly competed in the same assays (MA, third and fourth column) dnl2kol7 mutant males hardly courted at all (significantly less than in equal genotype assays) and wild type males displayed prolonged total courtship duration (B) dnl2-deficient males initiated courtship as frequently as wild types in equal genotype assays often but displayed reduced courtship initiation in direct competition with wild type males. (C) Individual courtship bouts of dni2-deficient males were longer than those of wild type reduced courtship initiation in direct competition with wild type males. (C) Individual courtship bouts of dnil2-deficient males were longer than those of wild type
males in equal genotype assays but shorter during direct competition in mixed genotype assays. (D) In assays with males of equal genotype (EA, first and second column) and during direct competition of males from both genotypes MA (third and fourth column) dnl2-deficient flies spent less time performing agonistic behaviours than wild type flies. (E) The tendency (no significance) to initiate aggressive encounters of dnl2-deficient males compared to wild type males was reduced in equal genotype assays but enhanced (due to low initiation frequency of wild types) during direct competition in mixed genotype assays. (F) If aggressive encounters were initiated in mixed genotype assays, their duration was significantly prolonged in wild type males. Each column contains data from $N=10$ socially naive males, analysis includes five periods of 1 min each from assays of 15 min duration. 'Represents $p<0.05$, “"represents $p<0.01$, ${ }^{* *}$ represents $p<0.001$, permutation tests on median differences.

To assess whether a reduced general activity or sensory impairments may cause the behavioural alterations of $d n l 2^{K 017}$ mutant flies, we performed several tests.

\subsection{General activity}

As a measure for general activity, we monitored the locomotor activity of male flies isolated in small glass tubes by counting the number of centre crossings over the course of four days under 12:12 light:dark cycles. Both wild type (compare with [36]) and dnl2-deficient flies exhibited rhythmic bimodal activity with peaks around light to dark and dark to light transitions (Fig. 4). There was no difference in the total number of centre crossings (median wild type $=2022(n=19)$; median $d n l 2^{K 017}=2294(n=20)$ ) but we observed a less pronounced oscillation between high activity phases coinciding with light changes and low activity periods in the middle of light and dark periods in dnl2-deficient compared to wild type males.

\subsection{Peripheral sensory processing}

Since $d n / 2$-deficient males selectively courted females and fought against males, processing of chemosensory information concerned with gender and sexual state recognition [37,38] appeared to be intact. To exclude potential impairments of peripheral visual pathways in $d n / 2^{K 017}$ mutant flies we recorded electroretinograms (ERGs) from the flies' compound eye surface. Comparison of ERGresponses (phasic-tonic responses of photoreceptors and phasic responses of lamina neurons [39]) to different intensities of white light stimulation revealed no differences between wild type and dnl2 ${ }^{K 017}$ mutant flies (Fig. 5A). In order to assess the functionality of the auditory system flies were acoustically stimulated with pulse song or pure tones at individual best frequencies of their aristae. Pulse songs were equally well represented in extracellular recordings from antennal nerves of wild type and $d n / 2^{K O 17}$ mutant males and no significant differences in auditory thresholds were detected (Fig. 5B) suggesting that the peripheral components of the auditory system are not compromised by the absence of Dnl2. 

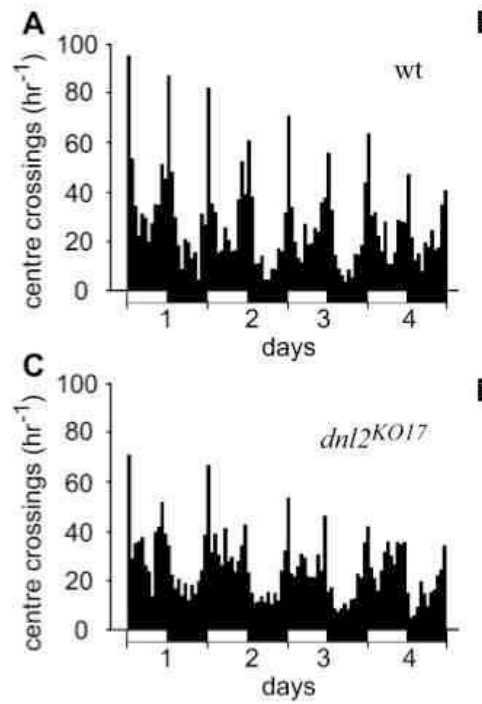

B.
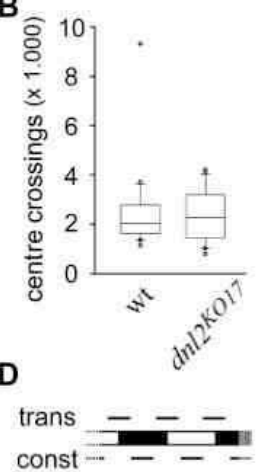

\begin{tabular}{l|c|c} 
& wt & KO17 \\
\hline trans & 1625 & 1406 \\
\hline const & 568 & 955 \\
\hline ratio & 2.86 & 1.47
\end{tabular}

Fig. 4. Locomotor activity of male wild type and dnl2-deficient D. melanogaster. (A and C) Average number of centre crossings of wild type (wt) $(A, n=19)$ and $d n 2^{\mathrm{Kol}}$. mutant $(C, n=20)$ males during four days under $12: 12 \mathrm{~h}$ light/dark cycles. Both genotypes show increased activity coinciding with light/dark and dark/light changes. (B) Total numbers of centre crossings during the four day observation period. Wild type and dnl2 $2^{\mathrm{KO} 17}$ mutant males are equally active. (D) Comparison of average individual activity between periods of six hours centering around light/dark and dark/light changes (trans) and six hours in the middle of light or dark phase (const) reveals less pronounced differences between "active" and "inactive" periods in $d n 12^{\mathrm{KO} 17}$ mutant compared to wild type males.

\section{Discussion}

Postsynaptic Neuroligins contribute to the formation of bidirectional trans-synaptic signalling complexes that promote preand postsynaptic differentiation and regulate synaptic functions. It is believed that disturbance of Neuroligin/Neurexin signalling promotes ASDs phenotypes through impairment of synaptic development, synaptic transmission and imbalance of excitatory and inhibitory synapses in brain circuits implicated in the regulation of social behaviour $[1,2,4,17,18]$. Both Neuroligins and Neurexins are evolutionary conserved [19] and orthologs of various proteins that associate with them to form functional pre- and postsynaptic complexes in the mammalian nervous system have been identified in invertebrate species. Neuroligin/Neurexin trans-synaptic signalling has been studied in rats and mice, the nematode $C$. elegans [9], the mollusc $A$. californica [40], the honeybee $A$. mellifera $[20]$ and at the neuromuscular junction of $D$. melanogaster $[21,22,41]$. Results of these studies suggest similar functions of Neuroligin/Neurexin signalling in the initiation, maturation, transmission and plasticity of vertebrate and invertebrate synapses.

Our study addressed the question whether impairment of Neuroligin/Neurexin trans-synaptic signalling impacts Drosophila's social behaviour and whether parallels to ASDs-like phenotypes reported in humans and mice (summarized in the introduction) could be detected. Neurexin-deficient males (described in [25]) displayed severe locomotor defects and were not able to produce courtship songs, though unilateral wing extension was occasionally observed (data not shown). A detailed analysis of neurexin-deficient flies was therefore not performed. In contrast. mutant lines deficient of the central nervously expressed $d n / 2$ displayed no obvious motor impairments and were able to produce both types of courtship song patterns with the same accuracy as wild-type Drosophila males, suggesting that the central pattern
A

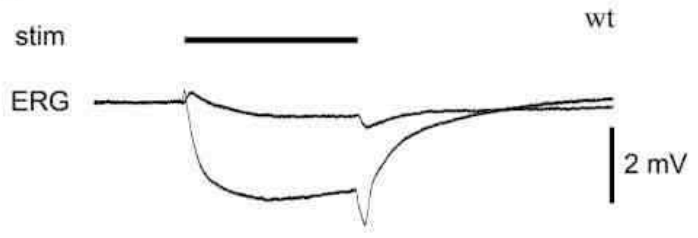

stim

$d n 12^{\mathrm{KOI7}}$

ERG

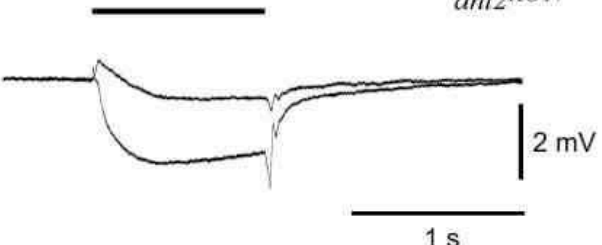

B

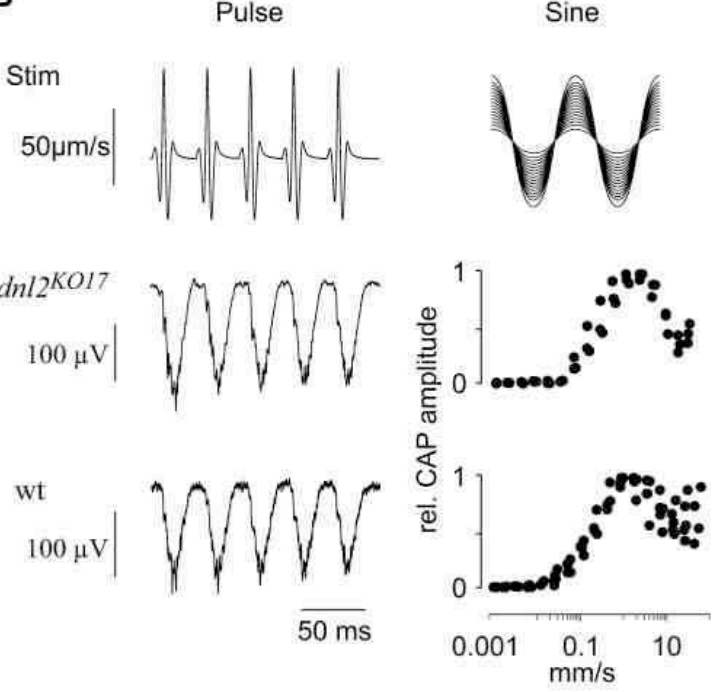

Fig. 5. Visual and auditory perception of male wild type and dnl2-deficient $D$. melanogaster (A) Electroretinogram (ERG) recordings: wild type (wt) and $d n 12^{\text {Ko1? }}$ mutant flies respond with typical ERG potentials of similar amplitudes to stimulation with white light (stim). ERG responses to identical low light intensity (upper traces in each diagram) and high light intensity (lower traces) stimuli are superimposed (each trace represents an average of three responses from the same fly). (B) Extracellular recordings of acoustically stimulated compound action potentials (CAPs) from the antennat no of $30 \mathrm{~ms}$; carrier frequency $170 \mathrm{~Hz}$ ) evokes correlated activity of similar amplitudes in the antennal nerve of wild type (wt) and dnl2k017 mutant flies. Right. Stimulation with pure sine signals of different intensities at the antenna's best frequency reveals no difference in response thresholds between wild type ( $N=5$ animals tested $)$ and dnl2-deficient $(N=3)$ flies, Each data point represents the average compound action potential amplitude of one fly to 10 stimuli.

generators for both pulse and sine song seem to function properly. Comparison of acoustic communication patterns of dnl2-deficient and wild-type flies revealed two differences, a reduced intensity of sine songs and shorter duration of inter pulse intervals. The reduced sine song intensity of $d n 2^{K 017}$ mutants likely results from a weaker synaptic transmission at the neuromuscular junction (reported by $[21,22])$ causing reduced muscle activation and lower amplitudes of wing vibrations. The reduced inter pulse interval must result from altered synaptic properties in thoracic pulse song pattern 
generating circuits and/or differences in the intensity of their activation by descending brain neurons [42]. The inter pulse interval is the critical parameter for species recognition and song attractiveness [33] and deviation from a species-typical range should reduce Drosophila's courtship success and reproduction. Altered ultrasound vocalization was also reported from mouse models for autism. While mice with impaired Neuroligin/Neurexin signalling displayed generally reduced calling rates $[12,15,43]$ other mouse strains with ASDs-like phenotypes displayed abnormal spectral and temporal song patterns [44]. Similar to the reduced rates of acoustic communication observed in mice with impaired Neuroligin/Neurexin trans-synaptic signalling, reduced courtship singing was also observed in our studies on dnl2-deficient Drosophila.

Distances between individuals of $D$. melanogaster have been studied in different behavioural settings which distinguished and emphasized different aspects including dispersal/exploration [45]. intrinsic social space [46-48] or group formation [49]. It has been demonstrated that inter individual space may depend on the balance of attractive and repulsive sensory signals, previous social experience like isolation or mating and also on the type of arena and the number of flies used for the assay (summarized in [48]). Our assay, which excluded exploration/dispersal during the first minutes after introduction into the arena, revealed that wild type flies initially establish shorter distances to conspecifics that steadily increase between 5 and $18 \mathrm{~min}$ after being placed in the arena, suggesting a gradually decreasing tendency to engage in short range interactions with other individuals. In contrast, dnl2-deficient flies displayed this low tendency, reflected in large inter individual distances, already after $5 \mathrm{~min}$ in the arena without showing consistent changes with progressing time in the arena.

Since $d n / 2$-deficient flies were equally active as wild type flies. displayed no sensory and motoric impairments, and were able to produce the typical components of courtship and agonistic behaviours, their reduced social interactions and impaired transition between different behaviours (e.g. from courtship singing to subsequent behaviour; between walking and turning) appear to result from altered information processing in central nervous circuits responsible for the initiation and coordination of behaviour. Especially the mushroom bodies and the central complex, that express Dnl2 (Chen and Xie, unpublished), have been implicated in these functions in insects $[50,51]$. Studies in the honeybee Apis mellifera revealed expression of various neuroligins and neurexin in the mushroom bodies [20] and regulation of brain neuroligin and neurexin expression by social interactions (comparison of isolated versus hive bees) during early adulthood [52]. This suggests that Neuroligin/Neurexin signalling may also be involved in behavioural plasticity resulting from social experience, which modulates the age-related division of labour in honeybee colonies. A similar relevance of activity-dependent neuroligin-and/or neurexin-mediated synaptic plasticity in the mature brain has also been documented in mice $[53,54]$ and has recently been implicated in the aetiology of ASDs [55].

Phenotypes very similar to those described here for $d n l 2$ deficient Drosophila have also been reported in various mouse models for ASDs including neuroligin-deficient mice. Behavioural and cognitive impairments of these mice, as well as ASDssymptoms in humans, have been linked to altered balance of excitation and inhibition in critical brain circuits that normally results from specific functions of different Neuroligin isoforms at excitatory and inhibitory synapses $[8,17,18]$ and altered functions of Neuroligin/Neurexin trans-synaptic signalling complexes [5]. Similarly, differential effects on synaptic properties are also mediated by $d n l 1$ and $d n l 2$ at the Drosophila neuromuscular junction $[21,22]$. Since $d n l 1$ is expressed in muscle but not in the nervous system, it will be interesting to see, whether $d n / 3$ or $d n l 4$ act as "balancing counterparts" to dnl2 within the nervous system to establish proper excitation/inhibition ratios. Circuits that regulate the initiation and intensity of social behaviours including acoustic communication may be especially sensitive to disturbances of neuroligin-mediated synaptic fine tuning and this sensitivity seems to be shared by humans, mice and Drosophila and probably other social insects like honeybees. Thus, phylogenetical conservation of Neuroligins from flies to humans extends beyond their molecular structure and their direct function at the synapse [19] and also includes their implication in the regulation of social behaviours.

With a huge battery of genetic tools available, Drosophila can serve as a useful model to further explore the contribution of Neuroligins and functionally associated molecules (e.g. neurexin and intracellular components of pre- and postsynaptic complexes) for synaptic functions, regulation of social behaviour and ASDs-related abnormalities.

\section{Acknowledgements}

We thank Margret Winkler and Stefanie Pauls for animal care, Christina Korthals for the execution of some behavioural experiments and Dr. Heribert Gras for advice concerning statistical analysis.

\section{References}

[1] Dean C, Dresbach T. Neuroligins and neurexins: linking cell adhesion, synapse formation and cognitive function. TINS 2006:29:21-9.

[2] Sudhof TC. Neuroligins and neurexins link synaptic function to cognitive disease, Nature 2008:455:903-11.

[3] Krueger DD, Tuffy LP, Papadopoulos T, Brose N. The role of neurexins and neuroligins in the formation, maturation, and function of vertebrate synapses. Current Opinion in Neurobiology 2012;22:412-22.

[4] Peca J, Feng G. Cellular and synaptic network defects in autism. Current Opinion in Neurobiology 2012;22:866-72.

[5] Arons MH, Thynne C]. Grabrucker AM, Li D, Schoen M, Cheyne JE, et al. Autism-associated mutations in ProSAP2/Shank3 impair synaptic transmission and neurexin-neuroligin-mediated transsynaptic signaling. Journal of Neuroscience 2012:32:14966-78.

[6] Wittenmayer N, Körber C, Liu H, Kremer T, Varoqueaux F, Chapman ER, et al. Postsynaptic neuroligin 1 regulates presynaptic maturation. Proceedings of the National Academy of Sciences of United States of America 2009:106:13564-9.

[7] Durand CM, Perroy J. Loll F. Perrais D, Bourgeron T. Montcouquiol M, et al. Durand CM, Perroy J. Loll F, Perrais D, Bourgeron T, Montcouquiol M, et al.
SHANK3 mutations identified in autism lead to modification of dendritic SHANK3 mutations identified in autism lead to modification of dendritic
spine morphology via an actin-dependent mechanism. Molecular Psychiatry 2012:17:71-84.

[8] Varoqueaux F, Aramuni G, Rawson RL, Mohrmann R, Missler M, Gottmann $\mathrm{K}$, et al. Neuroligins determine synapse maturation and function. Neuron 2006:51:741-54

[9] HuZ, Hom S, Kudze T, Tong X-J. Choi S, Aramuni G, et al. Neurexin and neuroligin mediate retrograde synaptic inhibition in C elegans, Science 2012;337:980-4.

[10] Baudouin S], Gaudias J, Gerharz S, Hatstatt L, Zhou K, Punnakkal P, et al, Shared synaptic pathophysiology in syndromic and nonsyndromic rodent models of autism. Science 2012:338:128-32.

[11] Jamain S, Quach H, Betancur C, Rastam M, Colineaux C, Gillberg IC, et al. Mutations of the X-linked genes encoding neuroligins NLGN3 and NLGN4 are associated with autism. Nature Genetics 2003:34:27-9.

[12] Jamain S, Radyushkin K. Hammerschmidt K, Granon S, Boretius S, Varoqueaux $\mathrm{F}$, et al. Reduced social interaction and ultrasonic communication in a mouse F, et al. Reduced social interaction and ultrasonic communication in a mouse
model of monogenic heritable autism. Proceedings of the National Academy of Sciences of United States of America 2008:105:1710-5.

[13] Radyushkin K, Hammerschmidt K, Boretius S, Varoqueaux F, El-Kordi A, Ronnenberg A, et al. Neuroligin-3-deficient mice: model of a monogenic heritable form of autism with an olfactory deficit. Genes, Brain and Behavior 2009;8:416-25.

[14] Ey E, Leblond CS, Bourgeron T. Behavioural profiles of mouse models for autism spectrum disorders. Autism Research 2011:4:5-16.

[15] Wöhr M, Silverman JL, Scattoni ML, Turner SM, Harris MJ. Saxena R, et al. Developmental delays and reduced pup ultrasonic vocalizations but normal sociability in mice lacking the postsynaptic cell adhesion protein neuroligin 2. Behavioural Brain Research 2012 (in press) http://dx.doi.org/10.1016/j.bbr.2012.07.024

[16] Rubenstein JL, Merzenich MM. Model of autism: increased ratio of excitation/inhibition in key neural systems. Genes, Brain and Behavior 2003:2:255-67.

[17] Chih B. Engelman H, Scheiffele B. Control of excitatory and inhibitory synapse formation by neuroligins. Science 2005:307:1324-8. 
[18] Han S. Tai C, Westenbroek RE, Yu FH, Cheah CS, Potter GB, et al. Autistic-like behaviour in $\mathrm{Scn}_{1 \mathrm{a}} \mathrm{i}_{-}$mice and rescue by enhanced GABA-mediated neuroransmission. Nature 2012:489:385-90.

[19] Knight D, Xie W, Boulianne GL Neurexins and Neuroligins: recent insights from invertebrates. Molecular Neurobiology 2011:44:426-40.

[20] Biswas S, Russell R], Jackson CJ. Vidovic M, Ganeshina O, Oakeshott JG, et al. Bridging the synaptic gap: neuroligins and neurexin $I$ in Apis mellifera. PLoS ONE 2008:1:e3542.

[21] Banovich D, Khorramshahi O, Owald D, Wichmann C, Riedt T, Fouquet W, et al. Drosophila neuroligin 1 promotes growth and postsynaptic differentiation at glutamatergic neuromuscular junctions. Neuron 2010:66:724-38.

[22] Sun M, Xing G, Yuan L, Gan G, Knight D. With SI, et al, Neuroligin 2 is required for synapse development and function at the Drosophila neuromuscular junction. synapse development and function at the

[23] Reiter LT, Potocki L. Chien S, Gribskov M, Bier E. A systematic analysis of human disease-associated gene sequences in Drosophila melanogaster. Genome Research 2001:11(6):1114-25.

[24] van Alphen B, van Swinderen B. Drosophila strategies to study psychiatric disorders. Brain Research Bulletin 2011, http://dx.doi.org/10.1016/j.brainresbull.2011.09.007 (in press).

[25] Zeng X, Sun M, Liu L, Chen F, Wei L, Xie W. Neurexin-1 is required for synapse formation and larvae associative learning in Drosophila, FEBS Letters 2007:581:2509-16.

[26] Fisher RA. Statistical methods for research workers. 12th ed. Edinburgh: Oliver and Boyd: 1954

[27] Crowley PH. Resampling methods for computation-intensive data analysis in ecology and evolution. Annual Review of Ecology and Systematics 1992:23:405-47

[28] Ernst MD. Permutation methods: a basis for exact inference. Statistical Science 2004:19:676-85.

[29] Benjamini Y, Hochberg Y. Controlling the false discovery rate: a practical and powerful approach to multiple testing. Journal of the Royal Statistical Society: Series B 1995:57:289-300.

[30] Groppe DM, Urbach TP, Kutas M. Mass univariate analysis of eventrelated brain potentials/fields I: a critical tutorial review. Psychophysiology 2011:48:1711-25.

[31] Effertz T, Wiek R, Gopfert MC. NompC TRP channel is essential for Drosophila sound receptor function. Current Biology 2011:21:1-6.

[32] Tauber E, Eberl DF. Acoustic communication in Drosophila. Behavioural Processes 2003:64:197-210.

133] Bennet-Clark HC, Ewing AW. Pulse interval as a critical parameter in the courtship song of Drosophila melanogaster. Animal Behaviour 1969:17:755-9.

[34] Jonsson T, Kravitz EA, Heinrich R. Sound production during agonistic behavior of male Drosophila melanogaster. Fly 2011:5:29-38.

[35] Chen S, Lee AY, Bowens NM, Huber R, Kravitz EA. Fighting fruit flies: a model system for the study of aggression. Proceedings of the National Academy of Sciences of United States of America 2002:99:5664-8.

[36] Helfrich-Förster C. Neurobiology of the fruit fly's circadian clock. Genes, Brain and Behavior 2005;4:65-76.

[37] Ferveur JF. Cuticular hydrocarbons: their evolution and roles in Drosophila pheromonal communication. Behavior Genetics 2005;35:279-95.
[38] Fernández MP, Chan YB, Yew JY, Billeter JC, Dreisewerd K, Levine JD, et al. Pheromonal and behavioural cues trigger male-to-female aggression in Drosophila. PLoS Biology 2010;8:e10000541.

[39] Heisenberg M. Separation of receptor and lamina potentials in the electroretinogram of normal and mutant Drosophila. Journal of Experimental Biology 1971:55:85-100.

[40] Choi YB, Li HL, Kassabov SR, Jin I, Puthanveettil SV, Karl KA, et al. Neurexinneuroligin transsynaptic interaction mediates learning-related synaptic remodelling and long-term facilitation in Aplysia. Neuron 2011:70:468-81.

[41] Li], Ashley J. Bhat MA Crucial role of Drosophila neurexin in proper active zone apposition to postsynaptic densities, synaptic growth, and synaptic transmission. Neuron 2007:55:741-55.

[42] Clyne JD, Miesenböck G. Sex-specific control and tuning of the pattern generator for courtship song in Drosophila. Cell 2008:133:354-63.

[43] Wöhr M, Roullet Fl, Hung AY, Ricceri L.. Crawley JN. Communication impairments in mice lacking Shank 1 : reduced levels of ultrasonic vocalizations and scent marking behaviour. PLoS ONE 2011;6:e20631.

[44] Scattoni ML, Gandhy SU, Ricceri L, Crawley JN. Unusual repertoire of vocalizations in the BTBR T+Hf] mouse model for autism. PLoS ONE 2008;3:e3067.

145] Simon JC, Dickson WB, Dickinson MH. Prior mating experience modulates the dispersal of Drosophila in males more than in females. Behavior Genetics 2011:41:754-67

[46] Bolduc FV, Valente D, Nguyen AT, Mitra PP. Tully T. An assay for social interaction in Drosophila fragile X mutants. Fly 2010;4:216-25.

[47] Simon JC, Dickinson MH. A new chamber for studying the behaviour of Drosophila. PLoS ONE 2010;5:e8793.

[48] Simon AF, Chou MT, Salazar ED, Nicholson T, Saini N. Metchev S, et al. A simple assay to study social behaviour in Drosophila: measurement of social space within a group. Genes, Brain and Behavior 2012;11:243-52.

[49] Schneider J. Dickinson MH, Levine JD. Social structures depend on innate determinants and chemosensory processing in Drosophila. Proceedings of the National Academy of Sciences of United States of America 2012:109:17174-9.

[50] Popov AV, Peresleni AI, Ozerskii PV, Shchekanov EE, Savvateeva-Popova EV. The role of the flabellar and ellipsoid bodies of the central complex of the brain of Drosophila melanogaster in the control of courtship behaviour and communicative sound production in males. Neuroscience and Behavioral Physiology 2005:35:741-50.

[51] Serway CN, Kaufman RR, Strauss R, de Belle JS. Mushroom bodies enhance initial motor activity in Drosophila. Journal of Neurogenetics 2009;23:173-84.

[52] Biswas S, Reinhard J, Oakeshott J. Russell R, Srinivasan MV, Claudianos C. Sensory regulation of neuroligins and neurexin $l$ in the honeybee brain. PLOS ONE sory regulation of
2010:5:e9133.

[53] Dahlhaus R, Hines RM, Eadie BD, Kannangara TS, Hines DJ, Brown CE, et al. Overexpression of the cell adhesion protein neuroligin-1 induces learning deficits and impairs synaptic plasticity by altering the ration of excitation to inhibition in the hippocampus. Hippocampus 2010:20:305-22.

[54] Schapitz IU, Behrend B, Pechmann Y, Lappe-Siefke C, Kneussel S], Wallace KE et al. Neuroligin 1 is dynamically exchanged at postsynaptic sites. Journal of Neuroscience 2010;30:12733-44.

[55] Ebert DH, Greenberg ME. Activity-dependent neuronal signalling and autism spectrum disorder. Nature 2013:493:327-37. 


\title{
7.3 Co-authorship publication 2
}

\section{A myelin gene causative of a catatonia- depression syndrome upon aging}

\author{
Nora Hagemeyer ${ }^{1 \dagger}$, Sandra Coebbels ${ }^{2 \dagger}$, Sergi Papiol ${ }^{1,3 \dagger}$, Anne Kästner ${ }^{1}$, Sabine Hofer ${ }^{4,5}$, \\ Martin Begemann ${ }^{1}$, Ulrike C. Gerwig ${ }^{2}$, Susann Boretius ${ }^{3,4}$, Georg L. Wieser ${ }^{2}$, \\ Anja Ronnenberg ${ }^{1}$, Artem Gurvich ${ }^{1}$, Stephan H. Heckers ${ }^{6}$, Jens Frahm ${ }^{3,4,5}$, \\ Klaus-Armin Nave ${ }^{2,3 * *}$, Hannelore Ehrenreich ${ }^{1,3 *}$
}

Keywords: anxiety; axonal degeneration; diffusion tensor imaging; low-grade inflammation; social withdrawal

DOI 10.1002/emmm.201200230

Received January 09, 2012 Revised February 09, 2012 Accepted February 13, 2012
Severe mental illnesses have been linked to white matter abnormalities, documented by postmortem studies. However, cause and effect have remained difficult to distinguish. CNP (2', $3^{\prime}$-cyclic nucleotide $3^{\prime}$-phosphodiesterase) is among the oligodendrocyte/myelin-associated genes most robustly reduced on mRNA and protein level in brains of schizophrenic, bipolar or major depressive patients. This suggests that CNP reduction might be critical for a more general disease process and not restricted to a single diagnostic category. We show here that reduced expression of CNP is the primary cause of a distinct behavioural phenotype, seen only upon aging as an additional 'pro-inflammatory hit'. This phenotype is strikingly similar in Cnp heterozygous mice and patients with mental disease carrying the AA genotype at CNP SNP rs2070106. The characteristic features in both species with their partial CNP 'loss-of-function' genotype are best described as 'catatoniadepression' syndrome. As a consequence of perturbed CNP expression, mice show secondary low-grade inflammation/neurodegeneration. Analogously, in man, diffusion tensor imaging points to axonal loss in the frontal corpus callosum. To conclude, subtle white matter abnormalities inducing neurodegenerative changes can cause/amplify psychiatric diseases.

\section{INTRODUCTION}

The CNP gene encodes the enzyme $2^{\prime}, 3^{\prime}$-cyclic nucleotide $3^{\prime}$ phosphodiesterase (CNP) which is present in non-compacted

(1) Division of Clinical Neuroscience, Max Planck Institute of Experimental Medicine, Göttingen, Germany

(2) Department of Neurogenetics, Max Planck Institute of Experimental Medicine, Göttingen, Germany

(3) DFG Research Center for Molecular Physiology of the Brain (CMPB), Göttingen, Germany

(4) Biomedizinische NMR Forschungs $\mathrm{GmbH}$, Max Planck Institute for Biophysical Chemistry, Göttingen, Germany

(5) Bernstein Center for Computational Neuroscience (BCCN), Göttingen, Germany

(6) Vanderbilt Department of Psychiatry, Nashville, TN, USA

*Corresponding author: Tel: +49 5513899 628; Fax: +49 5513899 670;

E-mail: ehrenreich@em.mpg.de

**Corresponding author: Tel: +49 5513899 757; Fax: +49 5513899 758;

E-mail: nave@em.mpg.de

'These authors contributed equally to this work. myelin areas such as the inner mesaxon, paranodal loops and Schmidt-Lantermann incisures (Braun et al, 2004; Yu et al, 1994), and accounts for about $4 \%$ of total central nervous system myelin proteins (Braun et al, 2004). CNP is expressed early in development of oligodendrocytes (Yu et al, 1994), increases with onset of myelination and remains detectable in these cells throughout life (Scherer et al, 1994). In vitro and in vivo studies demonstrated a regulatory function of CNP for process outgrowth in oligodendrocytes (Gravel et al, 1996; Lee et al, 2005; Yin et al, 1997), as well as an interaction with microtubules, cytoskeleton and RNA (Bifulco et al, 2002; De Angelis \& Braun, 1996; Gravel et al, 2009; Lee et al, 2005).

Studies employing homozygous Cnp-null mutant mice revealed that $\mathrm{Cnp}$ is essential for axonal survival but not for myelin assembly (Lappe-Siefke et al, 2003). In fact, Cnp-/mice show progressive axonal swellings and brain inflammation with first motor deficits occurring at 4 months that progress to severe hindlimb paralysis and death at 8-15 months (LappeSiefke et al, 2003). In contrast, Cnp $+/-$ mice with a $50 \%$ reduced $\mathrm{Cnp}$ expression do not exhibit any signs of inflamma- 
tion nor of abnormalities in neurological scoring or behaviour at least until the age of 12 months (Lappe-Siefke et al, 2003; Wieser et al, in preparation), indicating that lower Cnp levels can be fully compensated for

Nevertheless, decreased CNP expression could have pathophysiological significance. CNP is among the oligodendrocyte/ myelin-associated genes identified to be most robustly reduced both on mRNA and protein level in postmortem brains of schizophrenic, bipolar or major depressive patients (Aston et al, 2005; Mitkus et al, 2008; Tkachev et al, 2003). These findings suggest that CNP reduction might be critical in a more general disease process and that the potential role of this molecule is not restricted to a single diagnostic category but of global relevance for severe mental disorders.

Several genetic association studies have explored a potential impact of genetic variability in the CNP gene (chr17q21.2, $11 \mathrm{~Kb})$ on the overall risk for schizophrenia, with inconclusive results so far (Che et al, 2009; Peirce et al, 2006). Interestingly, however, a synonymous (Gly/Gly) single nucleotide polymorphism (SNP), localized in the fourth exon of the gene (rs2070106), influences CNP expression in the human cortex, especially in frontal areas, with the rarer A-allele showing lower expression in comparison to the G-allele (Iwamoto et al, 2008; Mitkus et al, 2008; Peirce et al, 2006).

Recent work indicates that in major psychiatric disorders like schizophrenia and depression, low-grade inflammation constitutes a crucial mechanism in the final common disease pathway (reviewed in Monji et al, 2009). Already the normal aging process is associated with slightly increased brain inflammation characterized by, for example, enhanced levels of pro-inflammatory cytokines, higher microglial numbers and increased reactivity with augmented expression of microglial surface markers (reviewed in, e.g. Miller \& Streit, 2007; Sparkman \& Johnson, 2008; Streit, 2006).

To address the pathophysiological relevance of reduced CNP expression, we chose CNP partial 'loss-of-function' genotypes with aging as an additional 'pro-inflammatory hit'. We examined old Cnp $+1-$ mice and schizophrenic patients with the AA versus GG genotype in the CNP SNP rs2070106. We report here the surprising association of CNP partial loss-offunction with a catatonia-depression syndrome both in mouse and man upon aging. Importantly, we provide evidence for lateonset low-grade inflammation in mice as a plausible pathophysiological mechanism. In patients carrying the low-expression genotype (AA), a comparable process might be reflected by axonal loss in the frontal corpus callosum as detectable by neuroimaging.

\section{RESULTS}

Brains of aging $\mathrm{Cnp}+\mathrm{I}-$ mice are characterized by enhanced inflammation, astrogliosis and axonal degeneration

Immunohistochemical analysis of mouse brains from age 4 to 26 months revealed an age-related increase in the number of ionized calcium-binding adapter molecule 1 (IBA-1) and Mac-3 positive microglia, infiltrating T-lymphocytes (cluster of differentiation 3; $\mathrm{CD} 3$ ) and astrocytes (glial fibrillary acidic protein, GFAP) in corpus callosum, striatum and anterior commissure (month 4 vs. month 26: all $p \leq 0.01$; for wild-type (Wt) as well as $\mathrm{Cnp}+/-$ mice). This increase was significantly more pronounced in old Cnp+/- as compared to Wt mice (Fig 1A-H). Axonal swellings (spheroids) as readout of neurodegeneration were determined in corpus callosum, striatum and anterior commissure using amyloid precursor protein (APP) immunoreactivity (Fig 1I/J). At the age of 4 months, no positive APP staining was detected. Thereafter, an age-dependent increase in axonal swellings became evident, again more prominent in Cnp+/ - mice (Fig 1I/J). Determination of Cnp mRNA expression in brains of young versus old mice revealed a remarkable decrease upon aging in Wt mice, which, however, still maintained levels above those in $\mathrm{Cnp}+\mathrm{/}-$ mice (Fig $1 \mathrm{~K}$ ). In both Wt and $\mathrm{Cnp}+/-$ mice, we found a corresponding age-dependent reduction of Cnp protein in purified myelin membranes, with the lowest overall level in aged Cnp $+/-$ (Fig 1L). Proteolipid protein (Plp), a control protein for compact myelin, also decreased with age but independent of the Cnp genotype (Fig 1L). Taken together, old $\mathrm{Cnp}+/$ - mice show a more pronounced low-grade inflammatory phenotype with axonal degeneration compared to Wt mice.

Aged $\mathrm{Cnp}+\mathrm{I}-$ mice have a slightly elevated anxiety profile but normal motor activity, coordination and strength

To test whether the pronounced histological changes upon aging are associated with any behavioural consequences, we investigated aged (24 months old) Cnp+/- and Wt mice employing a comprehensive test battery. In the open field test, a measure for general locomotor activity and anxiety, Cnp + /mice tended to spend less time in the centre than Wt $(p=0.096$; Fig 2A). Velocity and total distance travelled in the open field were comparable in both genotypes (Fig $2 \mathrm{~B}$ and $\mathrm{C}$ ), indicating normal activity. In the elevated plus maze, a classical anxiety test, open arm visits were reduced in Cnp + / - mice ( $p=0.036$; Fig 2D), whereas, the light/dark-box did not yield differences in the time spent in light (Fig 2E). Freezing behaviour is seen as another indicator of anxiety/fear in rodents. Cnp $+1-$ mice showed higher percentage of freezing in the fear conditioning chamber already at baseline, that is before measurement of conditioned or cued memory ( $p=0.007$; Fig $2 \mathrm{~F}$ ), precluding the use of fear conditioning for memory assessment in these mice. Like basic motor activity, which proved to be normal, motor performance, coordination and motor learning, as evaluated in a 2-day rota-rod testing, were not different between genotypes (Fig 2G). Also, gait analysis detected no motor abnormalities or ataxia (see, e.g. Fig $2 \mathrm{H}$ depicts forelimb stride of left and right paw) and muscle strength, measured by the grip strength test, did not differ between genotypes (Fig 2I). To summarize, 24 months old Cnp $+1-$ mice show normal overall motor performance and a mildly elevated anxiety profile in different anxiety-relevant tests compared to Wt mice.

\section{Aged Cnp+l- mice show impaired social and} exploratory behaviour

Social behaviour of aged Wt and Cnp+/- mice was tested in a three-partite chamber. This test measures the preference of a 

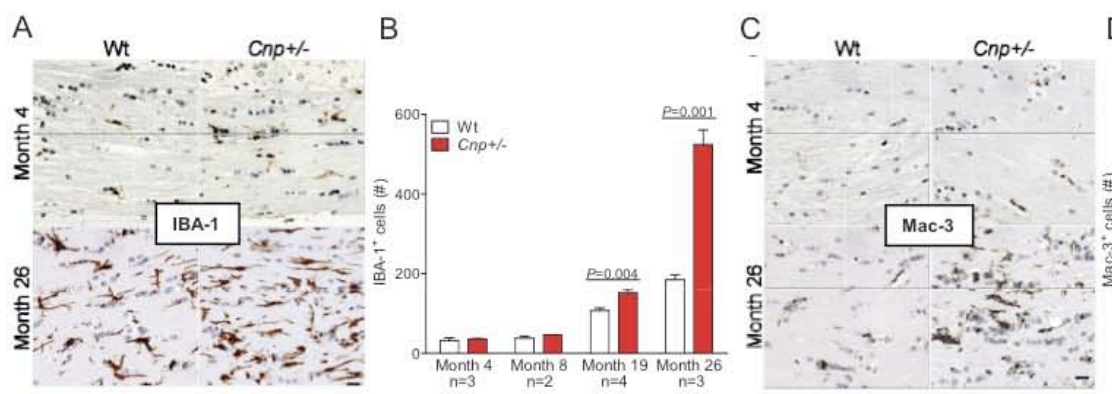

$\mathrm{D}$
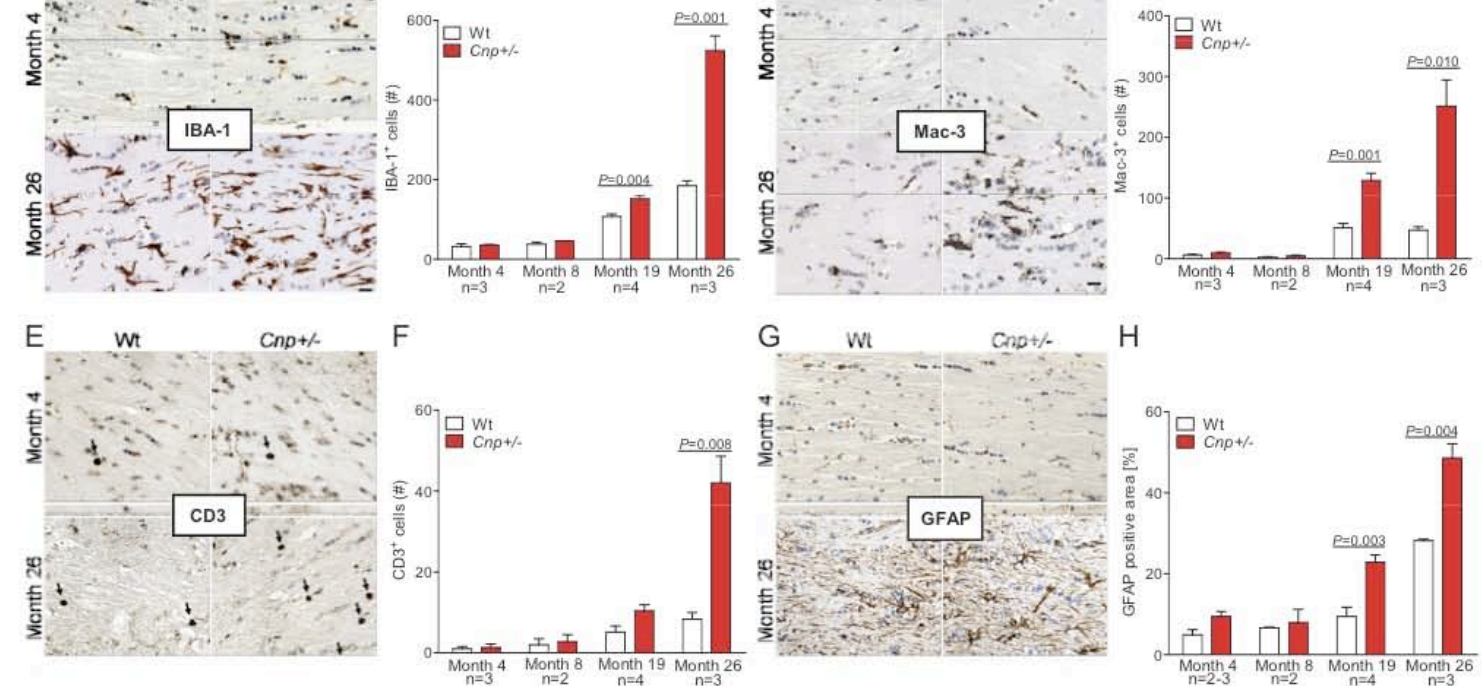

$$
J
$$
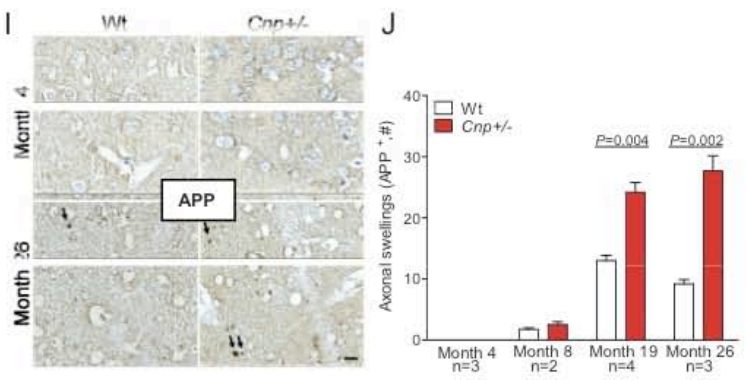

$\mathrm{K}$

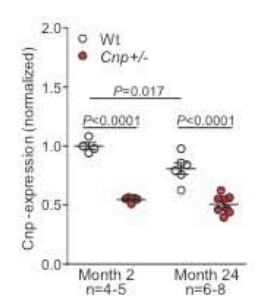

$\mathrm{H}$

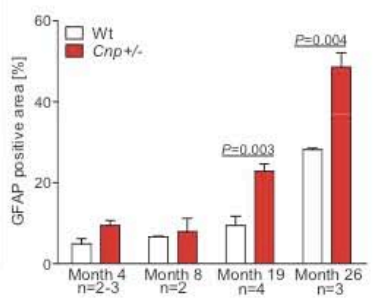

L

Wt Cnpt/-

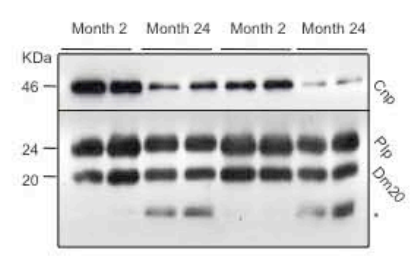

Figure 1. Low-grade brain inflammation and axonal degeneration in aged $\mathrm{Cnp}+\mathrm{I}-$ mice.

A. Representative microscopic images of the corpus callosum from 4 months (upper panels) and 26 months (lower panels) old Wt and $\mathrm{Cnp}+/-$ mice, immunostained for IBA-1; scale bar $20 \mu \mathrm{m}$.

B. Bar graph gives the age-dependent quantification of the total number of IBA-1 positive microglia in the corpus callosum of Wt and Cnp $+/-$ mice. For all quantifications (B, D, F, H, J), $n$ numbers indicated; mean \pm s.e.m. presented; two-sided Student's t-test used.

C. Representative microscopic images of the corpus callosum from 4 months (upper panels) and 26 months (lower panels) old Wt and Cnp $+/-$ mice, immunostained for Mac-3; scale bar $20 \mu \mathrm{m}$.

D. Bar graph gives the age-dependent quantification of the total number of Mac-3 positive microglia in the corpus callosum of Wt and Cnp+/- mice.

E. Representative microscopic images of the corpus callosum from 4 months (upper panels) and 26 months (lower panels) old Wt and $\mathrm{Cnp}+/-$ mice, immunostained for CD3; black arrows exemplify respective positive cells; scale bar $20 \mu \mathrm{m}$.

F. Bar graph gives the age-dependent quantification of the total number of $\mathrm{CD} 3$ positive T-lymphocytes in the corpus callosum, striatum and anterior commissure of Wt and Cnp+l- mice.

C. Representative microscopic images of the corpus callosum from 4 months (upper panels) and 26 months (lower panels) old Wt and Cnp $+/-$ mice, immunostained for GFAP; scale bar $20 \mu \mathrm{m}$.

H. Densitometrical quantification of the GFAP positive area in the corpus callosum.

I. Representative microscopic images of the striatum from 4 months (upper panels) and 26 months (lower panels) old Wt and Cnp $+/$ - mice, immunostained for APP; black arrows exemplify respective positive cells; scale bar $20 \mu \mathrm{m}$.

J. Bar graph gives the age-dependent quantification of the APP positive axonal swellings in the corpus callosum, striatum and anterior commissure of Wt and Cnp+l- mice.

K. Cnp mRNA expression level of Wt and Cnp+/- mice at months 2 and 24, normalized to mean value of ATP synthase subunit beta (Atp $5 b$ ) and acidic ribosomal phosphoprotein PO (RplpO) as housekeeper genes and to 2 months old Wt (1.0); mean \pm s.e.m. presented; two-sided Student's t-test used.

L. Cnp protein expression of Wt and Cnp $+/$ - mice at months 2 and 24, compared to Plp as control protein of compact myelin; " low-size band detected in aged brain myelin with the Plp antibody directed against the C-terminus of PLP/DM20. 


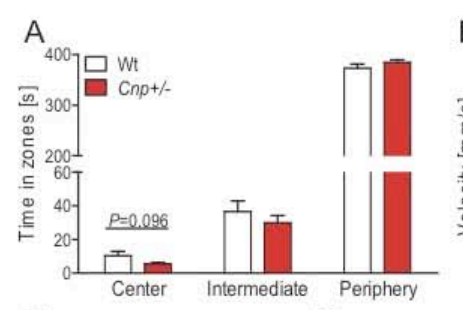

G

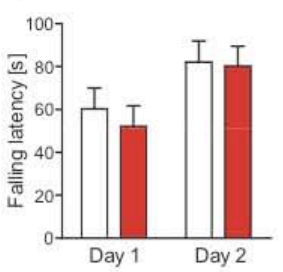

$\mathrm{H}$
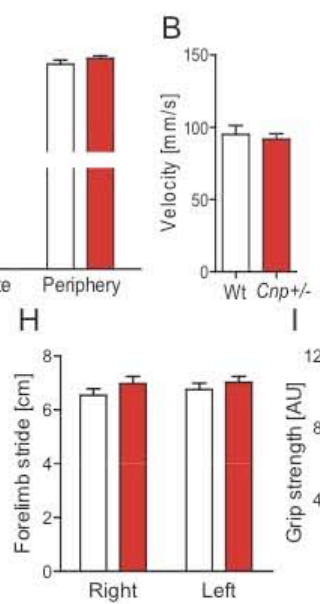

$\mathrm{N}$

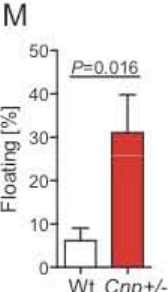

$\mathrm{N}$

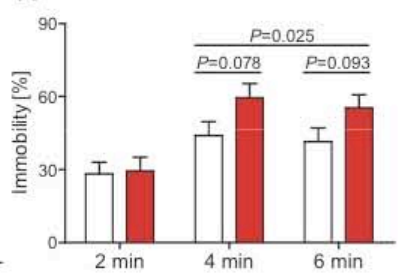

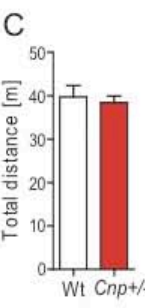
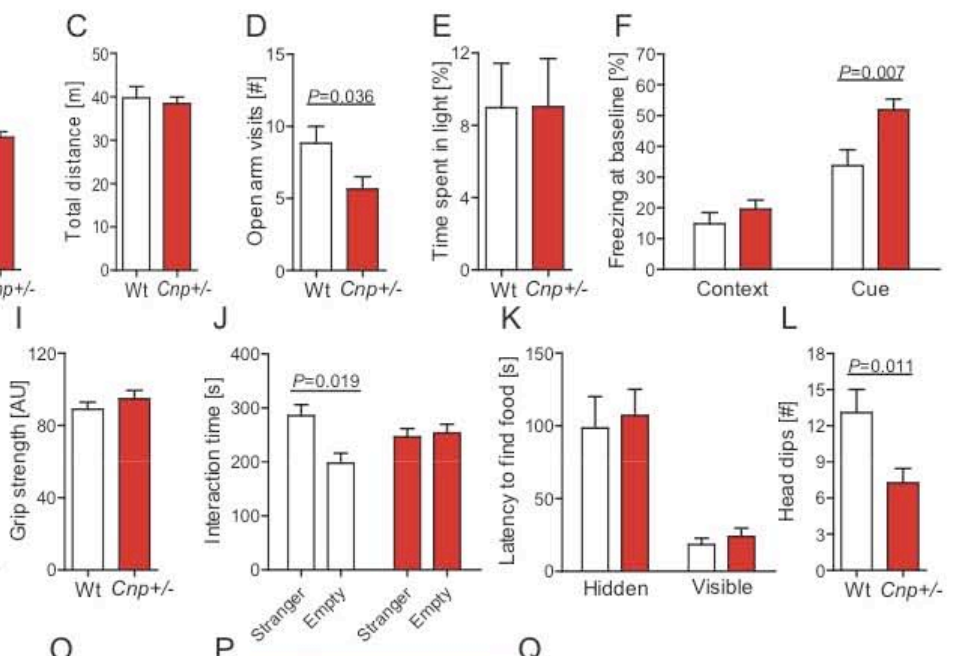

O

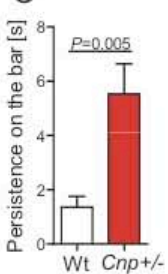

Q

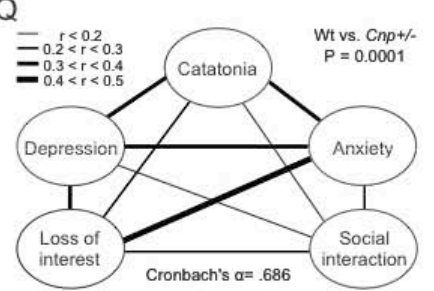

Figure 2. Aged $\mathrm{Cnp}+\mathrm{I}-$ mice show a phenotype composed of catatonia, depression, loss of interest, impaired social interaction and anxiety. A-C Open arm parameters.

D. Elevated plus maze.

E. Light/dark box paradigm.

F. Baseline freezing in the context and cue memory task of fear conditioning.

C. Rota-rod.

H. Gait analysis.

I. Grip strength

J. Sociability testing in the three-partite chamber.

K. Buried-food finding test - latency to find hidden versus visible food pellets.

L. Hole board.

M. Floating rate in a $90 \mathrm{~s}$ swim trial.

N. Tail suspension test.

O. Bar test for catatonia.

P. Typical posture of a catatonic Cnp + / - mouse during the bar test; see also videos of Supporting Information.

Q. Behavioural composite score displayed as intercorrelation network of Z-transformed items. Line thickness indicates the degree of correlation between 2 respective items. The composite score differs between genotypes $(p=0.0001)$. For all behavioural experiments, 24 months old mice were used: Wt $n=9-11$ and $C n p+I-n=10-16$; mean \pm s.e.m. presented; two-sided or paired $t$-tests used where applicable.

mouse for a chamber containing a small wire cage with a stranger mouse in comparison to a chamber with an empty wire cage. Aged Wt mice displayed the expected behaviour, that is spent significantly more time close to the cage with the stranger mouse compared to the empty wire cage $(p=0.019)$, whereas, Cnp $+1-$ mice did not show preference. To control for altered olfaction as a potential confounder of social behaviour in mice, the buried-food-finding test was performed, confirming normal olfactory function in both groups (Fig 2K). In the hole board test, measuring exploratory behaviour of mice, old $\mathrm{Cnp}+\mathrm{/}-$ mice had significantly less head dips ( $p=0.011$; Fig $2 \mathrm{~L}$ ), indicating loss of interest (in the absence of any signs of altered basic motor activity). To conclude, old Cnp $+/$ - mice demonstrate several facets of a loss of interest in the outside world.

\section{Aged $\mathrm{Cnp}+\mathrm{I}-$ mice exhibit features of depression and catatonia}

In the Morris water maze task, Cnp+/- mice displayed prominent floating behaviour, precluding analysis of this test for learning and memory. Analysis of the time mice spent floating within a swim trial of $90 \mathrm{~s}$ yielded threefold higher floating rates of $\mathrm{Cnp}+\mathrm{/}-$ mice in comparison to $\mathrm{Wt}$, which we interpret as a potential sign of depression ( $p=0.016$; Fig $2 \mathrm{M}$ ). To further consolidate this hypothesis, we performed an 
established test to measure depression in rodents, the tail suspension test, which determines over $6 \mathrm{~min}$ the time mice spend immobile. Fractionated analysis revealed that $c n p+/-$ mice had a higher duration of immobility in the second and last third of the test period compared to Wt ( $p=0.025$; Fig $2 \mathrm{~N}$ ), consistent with the typical 'give up' behaviour of depressed individuals. A phenotype, thus far observed in mice only upon induction (e.g. body pinch or drug exposure; Amir, 1986; Chaperon \& Thiebot, 1999) is catatonia/catalepsy, a state of immobility where mice persist in an externally imposed abnormal posture for a prolonged time period. Mice are put into a position where they have to grab a bar while standing with their hind paws on the floor (as illustrated in Fig 2P; for a striking example see videos of Supporting Information). Wt mice swiftly left this position, whereas, $\mathrm{Cnp}+/-$ mice persisted in this posture ( $p=0.005$; Fig 20). Taken together, old $\mathrm{Cnp}+/-$ mice exhibit a catatonia-depression syndrome.

\section{Creating a mouse behavioural composite, the 'catatonia- depression score'}

For translational purposes and confirmation of the internal consistency of our behavioural readouts in aged mice, we calculated intercorrelations between the observed behavioural sub-phenotypes catatonia, depression, loss of interest, impaired social interaction and anxiety as target variables. These variables, put together in a composite score, were internally consistent (Cronbach's $\alpha=.686$; Fig 2Q). Operationalization of the score items is detailed in the Materials and Methods Section. Expectedly, the score was significantly higher in Cnp + /$(0.32 \pm 0.44)$ than in Wt mice $(-0.43 \pm 0.41 ; p=0.0001)$. Based on these findings, we wondered whether reduced expression of the CNP gene in aging human patients may have a similar influence on the phenotype.

Exploiting the GRAS data base for a phenotype-based genetic association study on the role of CNP genotypes in a 'catatonia-depression syndrome'

To search for potential behavioural consequences of a previously described CNP loss-of-function genotype in humans (Iwamoto et al, 2008; Mitkus et al, 2008; Peirce et al, 2006), we conducted a phenotype-based genetic association study (PGAS) targeting the CNP SNP rs2070106 (A/G; Fig 3A) in $>1000$ schizophrenic patients of the Göttingen Research Association for Schizophrenia (GRAS) data collection (Begemann et al, 2010; Ribbe et al, 2010). As a first step, we performed a casecontrol analysis (schizophrenic patients vs. healthy controls) and found that this genetic marker does not contribute to an increased risk of schizophrenia in our population, as proven by the genotypic and the allelic chi-square comparison $(p>0.05$; Table I of Supporting Information).

Next, a composite score including all variables represented in the mouse behaviour composite was created that also yielded good internal consistency with a Cronbach's $\alpha=.695$ (Fig 3B). The operationalization of the score items is explained in the Materials and Methods Section. As illustrated in Fig 3C, the composite score shows a clear age and genotype (rs2070106) association: AA subjects develop a significantly higher score with increasing age as compared to GG carriers, with the dissociation of the regression lines starting at around the age of 40 years. We therefore set a cut-off of 40 years and focused on the older schizophrenic patients with our further PGAS analysis.

The characteristics of the GRAS patients with an age $\geq 40$ years, separated by AA versus GG genotype of rs2070106, are presented in Table 1. These data demonstrate that both genotype groups are comparable with respect to basic sociodemographic and clinical/disease control variables but differ highly significantly in the composite score measuring the catatonia-depression syndrome. Interestingly, heterozygote individuals (GA) are very similar to GG subjects. They do not show an intermediate phenotype in the composite score (Table II of Supporting Information). Importantly, when screening all items of the composite separately, a significant age-associated genotype (GG vs. AA) effect, comparable to the mouse findings, becomes evident for all (Fig 1 of Supporting Information).

CNP rs2070106 genotypes influence myelin/axon integrity in the frontal corpus callosum fibres, a candidate region of catatonia-depression

Based on clinical observation of the affected individuals - both mouse and man - and the scarce information in the literature on brain areas potentially involved in the catatonic phenomenon (Arora \& Praharaj, 2007; Northoff et al, 2004), we hypothesized that aging $\mathrm{AA}$ individuals displaying the catatonia-depression syndrome, in contrast to GG subjects, should show differences in axonal integrity of frontal crossing fibres. To prove this hypothesis, a subset of older patients of both genotypes (GG $n=11$; AA $n=10$ ) from the GRAS sample was selected and matched according to age, gender and duration of disease (Table 1). These patients, living all over Germany, were reinvited to Göttingen for diffusion tensor imaging (DTI). Indeed, DTI identified higher axial diffusivity (AD) and a higher apparent diffusion coefficient (ADC) in the frontal part of the corpus callosum (genu) of AA subjects as compared to GG patients ( $p \leq 0.005$ for both values; Fig 3E), consistent with a more progressed axonal loss/degeneration. This effect was specific for the frontal commissural fibres and was not observed in the posterior corpus callosum taken as a control region (Fig 3F). ADC values in the genu were generally correlated with age but, despite the small number of imaged subjects, resulted in a significant difference between genotypes upon linear discriminant analysis (LDA; $p<0.05$; Fig 3G). Importantly, there were no global brain volume differences detectable between GG and AA subjects that could have accounted for DTI results ( $p>0.05$ for all comparisons; Fig $3 \mathrm{H}$ ).

\section{DISCUSSION}

We report here the unexpected finding that CNP loss-of-function genotypes are causative of a mental syndrome, consisting of catatonia, depression, mild anxiety/social withdrawal, impaired social interaction and reduced interest in the outside world, which is remarkably similar in mouse and man. In both species, age becomes an important cofactor, supporting the view that the 
A

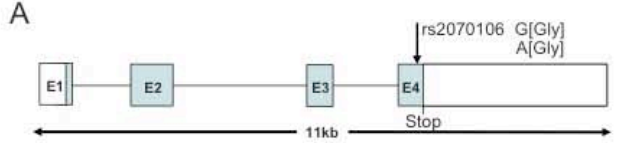

$\mathrm{B}$

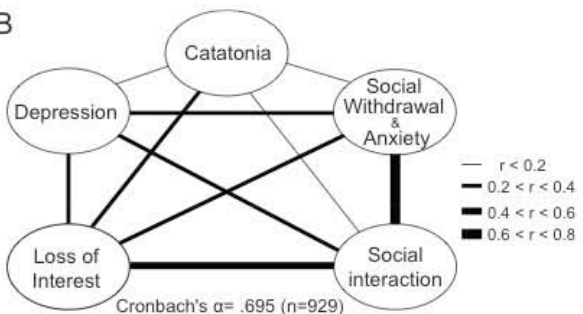

D

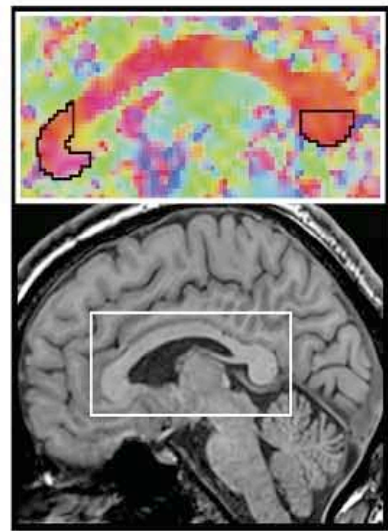

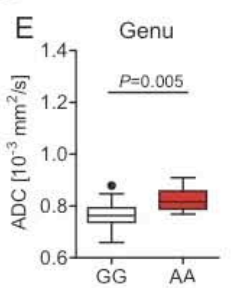

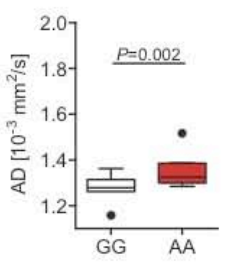

C
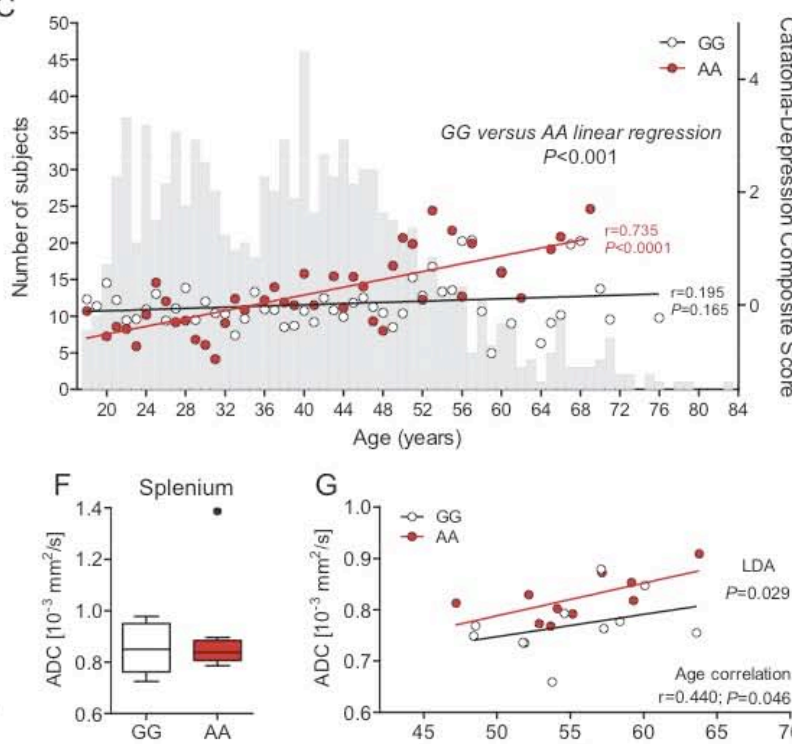

G
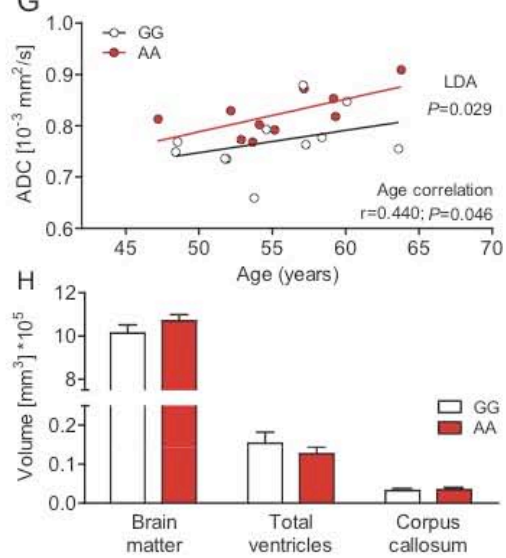

Figure 3. Age- and genotype-dependent association of the CNP rs2070106 SNP with a catatonia-depression syndrome in the GRAS sample of schizophrenic patients.

A. Schematic view of the human CNP gene structure and location of the synonymous SNP rs2070106 (A/G).

B. Intercorrelation network of all Z-transformed items of the catatonia-depression composite in the GRAS population. Line thickness indicates the degree of correlation between two respective items.

c. Correlation of genotypes with the catatonia-depression composite score across age groups. Grey bars in the background display the age distribution of the total GRAS sample of schizophrenic patients $(n=1048)$. Red or white circles denote mean values of the composite score for the respective age group and genotype (red, AA; black, GC). Linear regression lines of the genotypes dissociate after the age of 40 years. Pearson product-moment correlation applied.

D. Diffusion tensor imaging (DTI) study selecting the frontal (genu) and caudal (splenium) areas of the corpus callosum as regions of interest.

E,F ADC and AD values plotted according to rs 2070106 homozygosity status in genu (E, target region) and splenium ( $F$, control region) of the corpus callosum in a subgroup of schizophrenic individuals $>40$ years of age (CC $n=11$ and $A A n=10)$; results corrected for chlorpromazine equivalents (CPZ). Mean \pm s.e.m. presented and ANCOVA applied.

C. Correlation of ADC and age in AA and GG genotypes; linear discriminant analysis (LDA) with genotype as grouping variable and $A D C$ and age as independent variables. Pearson product-moment correlation applied.

H. Magnetic resonance imaging (MRI) volumetric comparison of brain matter, ventricular system and corpus callosum between genotypes. Mean \pm s.e.m. presented; two-sided Student's t-test applied.

underlying mechanism of this mental syndrome is a slowly progressive neurodegeneration, beginning in subcortical white matter, as described for the more rapid axonal loss in Cnp null mutant mice (Edgar et al, 2009; Lappe-Siefke et al, 2003). Importantly, the CNP loss-of-function genotype is causative of the here described behavioural syndrome but not of schizophrenia where it may only shape the aging phenotype.

In fact, the human part of this study has been obtained from a phenotypically extremely well characterized schizophrenic population (the 'GRAS data collection'), which was accessible and where all assessed items of the catatonia-depression syndrome are potentially relevant for disease subphenotypes. If a similar database on patients with, for example major depression had been available, the study would have been extended to this population. We expect that in individuals suffering from other mental disorders and even to some (perhaps mild) degree in healthy subjects, the phenotypical consequence of the CNP rs2070106 AA genotype will be 


\begin{tabular}{|c|c|c|c|c|c|}
\hline & \multicolumn{2}{|l|}{ GRAS sample $\geq 40$ years } & \multirow[t]{2}{*}{$p^{b}\left(F / X^{2}\right)$} & \multicolumn{2}{|l|}{ DTI subsample } \\
\hline & AA $(n=45)$ & GC $(n=235)$ & & AA $(n=10)$ & $\operatorname{GG}(n=11)$ \\
\hline \multicolumn{6}{|l|}{ Sociodemographic variables } \\
\hline Age, years, mean $\pm S D$ (range) & $51.04 \pm 7.65(40.44-69.93)$ & $50.16 \pm 8.42(44.03-79.49)$ & 0.515 & $52.07 \pm 4.74(44.08-60.30)$ & $49.50 \pm 5.34(40.66-58.71)$ \\
\hline Gender, No. $(\%)$, male & $26(57.8 \%)$ & $142(60.4 \%)$ & 0.740 & $7(70 \%)$ & $8(72.7 \%)$ \\
\hline Ethnicity, No. (\%), Caucasian & $43(95.6 \%)$ & $225(95.7 \%)$ & 0.892 & $10(100 \%)$ & $11(100 \%)$ \\
\hline $\begin{array}{l}\text { Years of education }{ }^{2} \text {. } \\
\text { mean } \pm S D \text { (range) }\end{array}$ & $12.27 \pm 3.82(0-21)$ & $12.32 \pm 3.36(0-27)$ & 0.933 & $12.45 \pm 3.39(9-19.5)$ & $14.14 \pm 3.16(8-19)$ \\
\hline \multicolumn{6}{|l|}{ Target variable } \\
\hline $\begin{array}{l}\text { Catatonia-depression composite } \\
\text { score, mean } \pm \text { SD (range) }\end{array}$ & $0.38 \pm 0.86(-1.13-1.91)$ & $0.03 \pm 0.74(-1.20-2.44)$ & $\begin{array}{l}0.009 \\
0.006^{\epsilon}\end{array}$ & $0.72 \pm 0.94(-0.99-1.85)$ & $0.02 \pm 0.76(-0.97-0.97)$ \\
\hline \multicolumn{6}{|l|}{ Clinical/disease control variables } \\
\hline $\begin{array}{l}\text { Age at first episode, years, } \\
\text { mean } \pm \text { SD (range) }\end{array}$ & $29.77 \pm 10.18(15.26-55.61)$ & $30.71 \pm 9.58(14.73-57.35)$ & 0.533 & $30.80 \pm 11.03(19.35-49.61)$ & $30.80 \pm 11.03(22.10-41.89)$ \\
\hline $\begin{array}{l}\text { Duration of disease (1st episode), } \\
\text { years, mean } \pm S D \text { (range) }\end{array}$ & $21.02 \pm 10.27(0.16-47.35)$ & $19.39 \pm 10.86(0.04-58.23)$ & 0.359 & $21.27 \pm 11.26(0.16-39.13)$ & $19.26 \pm 9.19(3.30-34.18)$ \\
\hline $\mathrm{CPZ}$, mean $\pm \mathrm{SD}$ (range) & $650.68 \pm 515.35(37.5-2295.0)$ & $805.76 \pm 915.14(0-7375.0)$ & 0.271 & $525.93 \pm 276.29(175-940)$ & $352.61 \pm 338.62(0-1200)$ \\
\hline PANSS pos, mean \pm SD (range) & $14.00 \pm 6.81(7-36)$ & $14.55 \pm 6.63(7-35)$ & 0.619 & $12.9 \pm 6.01(7-25)$ & $11.27 \pm 3.66(7-17)$ \\
\hline PANSS neg, mean $\pm S D$ (range) & $19.79 \pm 8.48(7-38)$ & $18.86 \pm 8.21(7-46)$ & 0.503 & $21.1 \pm 8.94(7-35)$ & $17.55 \pm 5.87(7-27)$ \\
\hline PANSS gen, mean $\pm S D$ (range) & $35.66 \pm 14.17(16-68)$ & $34.65 \pm 12.54(16-82)$ & 0.644 & $35.1 \pm 11.20(20-55)$ & $29.82 \pm 9.97(17-51)$ \\
\hline PANSS total, mean \pm SD (range) & $69.22 \pm 27.27(30-128)$ & $68.10 \pm 24.70(30-160)$ & 0.795 & $69.1 \pm 23.64(37-115)$ & $58.64 \pm 16.35(31-90)$ \\
\hline $\mathrm{CAF}$, mean $\pm S \mathrm{SD}$ (range) & $42.68 \pm 20.22(11-90)$ & $43.82 \pm 17.56(10-90)$ & 0.703 & $43.80 \pm 14.21(25-63)$ & $56.55 \pm 17.41(35-85)$ \\
\hline $\mathrm{CGl}$, mean $\pm \mathrm{SD}$ (range) & $5.75 \pm 1.35(3-8)$ & $5.62 \pm 1.13(2-8)$ & 0.488 & $6.00 \pm 0.94(5-7)$ & $5.09 \pm 1.14(3-7)$ \\
\hline
\end{tabular}

$\mathrm{CPZ}$, chlorpromazine equivalents as measure of antipsychotic drug dose; PANSS, positive and negative syndrome scale (consisting of three parts: pos; positive symptoms; neg, negative symptoms; gen, general psychopathology); GAF, global assessment of functioning; CGI, clinical global impression (see Ribbe et al, 2010 for further details).

Due to missing data upon phenotyping, sample size varies between $n=242$ and 280 in the sample of individuals with age equal to or above 40 years.

a Rating according to graduation/certificate; patients currently in school or in educational training are excluded.

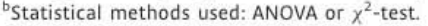

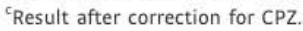

comparable. Along these lines, we show that many schizophrenic patients (and virtually all patients younger than 40 years) lack this syndrome. We would therefore like to stress again that this syndrome is independent of the diagnosis schizophrenia, which is also supported by the behavioural homology of the Cnp mouse model.

Several studies have suggested that schizophrenia and affective disorders are on a continuum of liability. Genetic linkage and association studies have proposed common disease loci for both disorders (Berrettini, 2000; O'Donovan et al, 2008). Family studies document that first-degree relatives of bipolar patients have a threefold higher risk for schizophrenia compared with first-degree relatives of healthy controls (Sham et al, 1994; Valles et al, 2000). Psychopathological syndromes, as the catatonia-depression syndrome shown here, shared by subgroups of both patient populations, would also be compatible with this overlap. Indeed, catatonia has been found to be highly prevalent in elderly patients with major depression (Starkstein et al, 1996). It will be interesting to determine whether depressed individuals that exhibit catatonic signs are also preferentially carriers of the CNP rs2070106 AA genotype.

To our knowledge, no spontaneous catalepsy in mice has as yet been reported, in contrast to pinch- or drug-induced catalepsy/catatonia (for review see, e.g. Amir, 1986; Chaperon \& Thiebot, 1999). The here observed Cnp+l- associated catalepsy/catatonia represents, therefore, the first clearly defined genetic catatonia model. Catatonia as a prominent phenotype has been extensively described by Karl Kahlbaum in 1874 (Kahlbaum, 1874) and entered the Diagnostic and Statistic Manual of Mental Disorders (APA, 2000) from its first edition in 1952 on, where it appears until now in connection with mood disorders, schizophrenia, and general medical conditions (Heckers et al, 2010). Nevertheless, reports on potential brain areas involved in this phenomenon in man are still scarce and point to frontal regions, based on, for example pronounced catatonia in a case with butterfly glioma of the frontal corpus callosum (Arora and Praharaj, 2007) or on a functional magnetic resonance imaging (MRI) study in akinetic catatonic patients during negative emotional stimulation (Northoff et al, 2004). We hypothesized that genotype-dependent axonal degeneration should be detectable in the frontal commissural fibres of the corpus callosum. These considerations were supported by the fact that the catatonia presented here in the context of a syndrome is characterized by several features of a primarily executive control (frontal lobe) deficiency in the absence of any 'classical' motor dysfunction. Indeed, we could localize axonal degeneration, determined by an increased axonal diffusivity in DTI, selectively to the genu corporis callosi.

The CNP rs2070106 AA genotype leads to reduced expression of CNP (Mitkus et al, 2008; Peirce et al, 2006), constituting 'partial loss-of-function'. Since there is an appreciable degree of linkage disequilibrium across the CNP gene (www.hapmap. org), the effects seen with the synonymous SNP rs2070106 might well be due to the influence of another genetic variant in 
close vicinity (e.g. in the $3^{\prime}$-untranslated region ( $3^{\prime}$-UTR) of the CNP gene). Alternatively, according to previous studies, synonymous SNPS may modify translational timing due to differential codon usage (Kimchi-Sarfaty et al, 2007) or inactivate an exonic splicing silencer that compensates for other genetic variations in exonic splicing enhancers (Nielsen et al, 2007).

We demonstrated increased numbers of inflammatory cells, gliosis and axonal degeneration in old $\mathrm{Cnp}+/$ - mice suggesting an important role of low-grade inflammation in the described syndrome. Even though brain sections of human patients with the respective CNP genotypes were not available in the present study, the axonal degeneration detected by DTI is an intriguing observation that might point to the hypothesis of a comparable disease mechanism in mouse and man. Mechanistic details on the subcellular functions of CNP in myelinating oligodendrocytes have been reported (Gravel et al, 2009) and are under further investigation. The secondary neuroinflammation is a well-known cause of nitric oxide-mediated axonal stress and neurodegeneration (for review see Amor et al, 2010; Smith \& Lassmann, 2002). We note that a diverse group of inherited myelinopathies in the nervous system of mice can trigger the recruitment of microglia/macrophages and T-cells (Ip et al, 2006; Kassmann et al, 2007; Martini \& Toyka, 2004), demonstrating that low-grade inflammation is a rather unspecific response of myelinating glial cells to cellular stress, possibly related to perturbed lipid metabolism (Dumser et al, 2007). Interestingly, low-grade inflammation has been found to be associated with behavioural consequences in mouse studies (Bercik et al, 2010) and hypothesized to play a role in mental diseases (Gardner \& Boles, 2011; Monji et al, 2009; Muller \& Schwarz, 2008; Schnieder \& Dwork, 2011). Respective first clinical trials employing antiinflammatory strategies in bipolar disease and schizophrenia yielded positive signals (Laan et al, 2010; Muller et al, 2010). Having information available on a predisposing genotype, individualized preventive and therapeutic approaches may be possible in the future.

To conclude, the major finding of the present study is the proof-of-principle that subtle changes of subcortical white matter can be the cause, rather than merely the consequence, of a complex neuropsychiatric syndrome. This distinction is extremely difficult in human patients with a psychiatric disease of unknown etiology, specifically when pharmacologically treated and only diagnosed (by MRI) with minor abnormalities of white matter tracts (Davis et al, 2003). Our analysis was possible by building on genetic variants of the cell type-specific CNP gene that lead to a partial loss-of-function genotype in both mouse and man. Importantly, Cnp heterozygosity (in mice) and moderately reduced $C N P$ expression levels (in humans) are well tolerated until an advanced age. At that point, however, haploinsufficiency causes a striking phenotype in mice and shapes the phenotype of a complex psychiatric disease in humans. Although we have no ultimate proof that moderately reduced CNP levels in any individual (diseased or healthy) suffice to trigger a catatonia-depression syndrome upon aging, they clearly add to other genetic factors (here in patients diagnosed with schizophrenia) such that the catatonia-depression syndrome can be well defined and emerges as remarkably similar to the isolated behavioural phenotype of aged Cnp heterozygous mice. This amazing similarity of the behavioural phenotype in two different species emphasizes the relevance of glial dysfunction in psychiatric disorders, and supports the exploration of therapeutic strategies to target the associated low-grade neuroinflammation.

\section{MATERIALS AND METHODS}

\section{Human studies}

\section{Healthy subjects}

Blood donors ( $n=1045$; Begemann et al, 2010) were recruited for the case-control study. Ethnicity (Caucasian $97.8 \%$; other ethnicities $2 \%$; unknown $0.2 \%$ ) was comparable to the patient population (Caucasian $95.5 \%$; other ethnicities $1.8 \%$; unknown $2.7 \%$ ).

\section{Schizophrenic patients}

The GRAS data collection was approved by Ethics Committees of the Georg-August-University of Göttingen and participating centres, and comprises at present 1048 patients with Diagnostic and Statistical Manual of Mental Disorders-IV (DSM-IV; APA 2000) diagnosis of schizophrenia $(81.7 \%)$ or schizoaffective disorder $(18.3 \%)$, examined between 2005-2010 in 23 centres all over Germany (for details see Ribbe et al, 2010). Interviews, testing and ratings were conducted by an invariable team of trained examiners using the 'GRAS Manual' (Begemann et al, 2010; Ribbe et al, 2010).

\section{Catatonia-depression composite}

The score consists of five phenotype domains: Depression was operationalized by items 3 (guilt feelings) and 6 (depression) of general psychopathology subscale of Positive and Negative Syndrome Scale (PANSS) (Kay et al, 1987). Catatonia was based on catatonic signs subscale of the Cambridge Neurological Inventory (gait mannerism, gegenhalten, mitgehen, imposed posture, abrupt or exaggerated spontaneous movements, iterative movements, automatic obedience and echopraxia; Chen et al, 1995). Deficits in social interaction were built on items 1 (blunted affect) and 3 (poor rapport) of PANSS negative subscale, combined with item 44 (never feeling close to another person) of Brief Symptom Inventory (Derogatis \& Melisaratos, 1983). Social withdrawallanxiety was assessed by item 4 (social withdrawal) of PANSS negative subscale and item 12 (suddenly scared for no reason) of Brief Symptom Inventory. Loss of interest in the outside world was estimated by item 7 (self-centred attitude) of PANSS negative and item 15 (preoccupation) of general subscale. Phenotype domains were $\mathrm{Z}$-standardized to be normally distributed with expectation zero and variance one. Higher values indicate worse outcome. Composite calculation was based on subjects without missing data $(n=929)$. Correlations of the five target phenotypes were assessed using Pearson product-moment correlation and internal consistency was determined using Cronbach's $\alpha$.

\section{Genotyping}

Genotyping of SNP rs2070106 was performed using SimpleProbes (TIB Molbiol, Berlin, Germany) on LightCycler480 (Roche Diagnostics, Basel, Switzerland). 
Research Article

CNP genotypes are associated with catatonia-depression

$M R I / D T I$

For MRI/DTI analyses, a subset of patients $\geq 40$ years of both genotypes (CG $n=11 ;$ AA $n=10$ ) from the GRAS sample was selected and matched according to age, gender and duration of disease. Studies were conducted at $3 \mathrm{~T}$ (Tim Trio, Siemens Healthcare, Erlangen, Germany) using a 32-channel head coil. DTI was performed at $2 \mathrm{~mm}$ isotropic resolution using diffusion-weighted single-shot stimulated echo acquisition mode (STEAM) sequences (Hofer et al, 2010; Karaus and Frahm, 2009) combining 6/8 partial Fourier encoding and parallel imaging. Protocol comprised 24-independent diffusion gradient directions and $b$-values of 0 and $900 \mathrm{smm}^{-2}$. A total of 55 transverse sections ( $2 \mathrm{~mm}$ thickness) covered brain parts dorsal and ventral to the corpus callosum. To increase signal-to-noise ratio, acquisition was repeated three times (17 min). Anatomic images were based on $T_{1}$-weighted 3D fast low angle shot (FLASH) MRI sequence (repetition time $T R=11 \mathrm{~ms}$, echo time $T E=4.9 \mathrm{~ms}$, flip angle $15^{\circ}$ ).

\section{$D T I$ regions of interest $(R O I)$}

Before calculation of diffusion tensor, diffusion-weighted MRI data sets were interpolated to $1 \mathrm{~mm}$ isotropic resolution and smoothed with a 3D Gaussian filter (half width $1 \mathrm{~mm}$ ). Individual ROIs were manually defined on colour-coded maps of the main diffusion direction without thresholding. ROIs for the corpus callosum were placed in the midsagittal plane as well as in two directly neighbouring parasagittal sections covering central portions of genu and most posterior part of splenium (Hofer \& Frahm, 2006). Mean values of fractional anisotropy (FA), $A D C, A D$ and radial diffusivity (RD) were calculated.

\section{MRI volumetry}

Analyses were performed with an automatic brain segmentation tool for surface-based cortical thickness (http://surfer.nmr.mgh.harvard.edu). $T_{1}$-weighted images underwent corrections for intensity inhomogeneity, skull strip and registration into Talairach space followed by segmentation into grey matter, white matter and various brain areas. Regional differences of cortical thickness between patient groups were investigated using Qdec (FreeSurfer for multiple comparisons and voxel-based morphometry). Statistics relied on $p \leq 0.05$ (false discovery rate corrected for multiple comparisons). Visualization employed an inflated pial surface model.

\section{Mouse studies}

\section{Mouse mutants}

Experiments were carried out according to animal policies of the German Federal State of Niedersachsen. Cnp+1- mice were genotyped with primers Cnp-E3s, $5^{\prime}$-GCCTTCAAACTCTCCATCTC-3'; CnpE3as, 5'-CCCACCCCTTTTATTACCAC-3' and puro3, 5'-CATACCCTCAAGAACGAGA- $3^{\prime}$.

\section{Immunostaining}

Mice were anesthetized with Avertin (Sigma-Aldrich, Taufkirchen, Germany) and perfused through the left ventricle with $15 \mathrm{ml}$ of Hank's balanced salt solution (Lonza, Basel, Switzerland), followed by $50 \mathrm{ml}$ of $4 \%$ paraformaldehyde in phosphate buffered saline (PBS). Brains were harvested and postfixed in $4 \%$ paraformaldehyde overnight at $4{ }^{\circ} \mathrm{C}$ and then embedded in Paraplast (Surgipath Paraplast; Leica, Wetzlar,
Germany). Microtome sections of $5 \mu \mathrm{m}$ (Microm HM400, Walldorf, Germany) were prepared. For diaminobenzidine (DAB)-based immunostaining of paraffin sections, Dako- $\mathrm{LSAB}_{2}$ system or Vectastain Elite $A B C$ kit (Vector laboratories, Burlingame, $C A$, USA) were used according to manufacturer's instructions. Primary antibodies were directed against APP (1:750, Chemicon (Millipore) Billerica, MA, USA), CD3 (1:150, Serotec, Oxford, UK), GFAP (1:200, Novocastra (Leica) Newcastle Upon Tyne, UK), IBA-1 (1:1000, Wako, Neuss, Germany) and Mac-3 (1:400, BD Pharmingen, Franklin Lakes, NJ, USA).

\section{Quantitative real time polymerase chain reaction ( $q R T-P C R$ )}

qRT-PCR was performed using SYBR green master mix (Applied Biosystems, Foster City, CA, USA) and 7500 Fast Real-Time PCR System (Applied Biosystems). Specific qRT-PCR primers were designed by Roche Universal ProbeLibrary Assay Design Center (Cnp, forward 5'TAACCCTCCCTTAGCCCCTG-3', reverse 5'-GTCCCTACCATGTCGCACCT-3'; for normalization: Atp5b forward 5'-GCATCTCCTGGCCCCATAC-3', reverse 5'-CTTTCCAACGCCACCACCT-3', Rplpo forward 5'-GATCCCCAGGGAAGACAG-3', reverse 5'-ACAATGAAGCATTTTGGATAATCA-3'). Data were analysed with Microsoft Excel 2010.

\section{Western blot}

Myelin purified from protein lysates was performed according to (Norton \& Poduslo, 1973). For Western blotting, proteins were sizeseparated in $12 \%$ sodium dodecyl sulphate (SDS)-polyacrylamide gels $(0.2 \mu \mathrm{g} / \mu \mathrm{l})$, blotted onto polyvinylidene difluoride membranes (Hybond P; GE Healthcare, München, Germany), blocked with $5 \%$ milk powder in Tris-buffered saline (TBS) and Tris-buffered saline + Triton X100 (TBST; $150 \mathrm{mM} \mathrm{NaCl}, 10 \mathrm{mM}$ Tris/HCl, pH 7.4; 0.1\% Tween20), and incubated with primary antibodies (CNPase, 1:5000, Sigma, Saint Louis, MO, USA; Plp (A431), 1:5000; Jung et al, 1996), overnight at $4^{\circ} \mathrm{C}$. Blots were washed with TBS/0.05\% Tween 20 , incubated with appropriate secondary horseradish peroxidase-conjugated antibodies (Dianova, Hamburg, Germany), washed with TBS/0.05\% Tween 20 and developed by enhanced chemiluminescence (Pierce, Rockford, IL, USA).

\section{Morphometry}

Digitized overlapping light microscopic images (20x if not otherwise stated), fused to a continuous image of a complete corpus callosum (bregma $0.74 \mathrm{~mm}$ ) by using Photoshop CS5 and Imagej software were analysed for absolute numbers of IBA-1 and Mac-3 positive cells. To quantify GFAP positive areas, a plug-in for the Imagej software for semi-automated analysis was implemented (http://www1.em.mpg.de/ wieser). APP positive axonal spheroids (analysed at $40 \times$ magnification) and $C D 3$ positive T-cells are expressed as total numbers quantified in corpus callosum, anterior commissure and striatum. For all stainings, two sections per mouse were quantified.

\section{Behavioural testing}

Tests were performed as described in detail previously, using the following order: Elevated plus maze (Radyushkin et al, 2010), open field (Radyushkin et al, 2010), light/dark box (Finn et al, 2003), rota-rod (Radyushkin et al, 2010), gait analysis (Brooks \& Dunnett, 2009), grip strength (Radyushkin et al, 2010), hole board (Radyushkin et al, 2009), sociability (Moy et al, 2004), buried-food-finding test for olfaction (Radyushkin et al, 2009), floating behaviour (analysis of swimming/ floating during a 90 s trial in the Morris water maze pool; Morris, 1984; 


\section{The paper explained}

PROBLEM:

Myelin and white matter abnormalities have been documented in neuropsychiatric diseases such as schizophrenia, major depression and bipolar disorder. However, their significance for disease mechanisms, pathogenesis or phenotypes is still obscure. A considerable number of postmortem studies found reduced expression of several myelin genes, including $2^{\prime}, 3^{\prime}$-cyclic nucleotide $3^{\prime}$-phosphodiesterase (CNP), in the brains of individuals with severe mental disease. In the present translational approach, we report for the first time phenotypical consequences of moderate CNP 'loss-of-function' genotypes, that is genetic variants leading to decreased CNP expression, both in man (single nucleotide polymorphism rs2070106) and mouse (heterozygous Cnp null mutant mice).

\section{RESULTS:}

We show that reduced CNP expression causes a distinct behavioural abnormality, seen only upon aging as an additional 'pro-inflammatory hit'. This phenotype is strikingly similar between $C_{n p}$ heterozygous mice and patients with mental disease, carrying the AA genotype at CNP SNP rs2070106. The characteristic features in both species are best described as a 'catatonia-depression' syndrome and include bizarre posturing, depression, anxiety, loss of interest in the outside world and social withdrawal. As a consequence of perturbed CNP expression, mice show secondary low-grade inflammation and degeneration of nerve fibres. Analogously, in man, diffusion tensor imaging points to axonal loss in the frontal corpus callosum.

\section{IMPACT:}

Our genetic data demonstrate that subtle white matter abnormalities can be the cause of a psychiatric syndrome. To our knowledge, CNP is the first gene identified to be associated with catatonia, and aged heterozygous null mutant mice are the first animal model of spontaneous catatonia. Moderately reduced CNP expression contributes to a distinct phenotype, which is not restricted to a single diagnostic category but could explain features of catatonia-depression in different mental disorders and possibly - to a milder degree - even in aging healthy individuals. This knowledge will help defining subgroups of (aging) subjects who may profit from novel, more specific therapeutic approaches including anti-inflammatory strategies.
Stone \& Lin, 2011), tail suspension test (Cryan et al, 2005), bar test (Kuschinsky \& Hornykiewicz, 1972; see Fig 2P and videos of Supporting Information) and fear conditioning (Radyushkin et al, 2009).

\section{Mouse score}

For the catatonia-depression score, five phenotype domains were created: (1) Depression was operationalized by floating time and tail suspension (delta time of immobility in the last 2 min minus first $2 \mathrm{~min}$ ), (II) catatonia by time on bar, (III) deficit in social interaction by delta time spent with stranger versus empty compartment, (IV) anxiety by open field-duration in centre, elevated plus maze - open arm visits, and fear conditioning - freezing at baseline in cue task and $(V)$ loss of interest by hole board - number of head dips. Composite score calculation was done in analogy to the human score and based on mice with not more than two variables missing $(n=27)$.

\section{Statistical analysis}

Statistical analyses were performed using SPSS for Windows version 17.0 (https://www.spss.com/de) and Prism5 (GraphPad Software, San Diego, CS, USA). Exact procedures are indicated in the respective sections. Note: All experiments/analyses in both men and mice were performed by persons unaware of genotypes ('blinded').

\section{Author contributions}

NH, SG, SP, KAN and HE developed study concept and design; $\mathrm{NH}$ performed all behavioural analysis of $\mathrm{Cnp}+/$ - mice; SP and AK carried out human genetic analyses and performed the human association study; UCG and GLW performed the histological analyses of Cnp+/- mice under supervision of SG; DTI study with human subjects was performed by SH and analysed under the supervision of SB and JF; Administrative, technical and material support was provided by MB, AR and AG in different aspects of the study; $\mathrm{MB}$ and $\mathrm{AK}$ coordinated and supervised the recruitment of subjects for the DTI study; SHH, SB and JF gave input to data analysis, interpretation, and manuscript preparation; NH, SG, SP, AK, KAN and HE wrote the manuscript; KAN and HE had full access to all of the data in the study and take responsibility for the integrity of the data and the accuracy of the data analysis.

\section{Acknowledgements}

We are indebted to all patients for their participation in the GRAS (Göttingen Research Association for Schizophrenia) study and all collaborating GRAS centres for their support. We are grateful to all colleagues who contributed to the GRAS data collection. This work was supported by the Max Planck Society, the DFG-Research Center for Molecular Physiology of the Brain (CMPB) and the Bernstein Center for Computational Neuroscience (BCCN) (Grant 01GQ0431). K.A.N. acknowledges grant support from ERA-Net Neuron (Grant 01EW1102) and is recipient of an ERC Advanced Grant (Axoglia).

Supporting Information is available at EMBO Molecular Medicine online. 
Research Article

CNP genotypes are associated with catatonia-depression

The authors declare that they have no conflict of interest.

\section{References}

Amir S (1986) Catalepsy induced by body pinch: relation to stress-induced analgesia. Ann N Y Acad Sci 467: 226-237

Amor S, Puentes F, Baker D, van der Valk P (2010) Inflammation in neurodegenerative diseases. Immunology 129: 154-169

APA. (2000) Diagnostic and Statistical Manual of Mental Disorders: DSM-IV-TR, Washington, American Psychiatric Association.

Arora M, Praharaj SK (2007) Butterfly glioma of corpus callosum presenting as catatonia. World J Biol Psychiatry 8: 54-55

Aston C, jiang L Sokolov BP (2005) Transcriptional profiling reveals evidence for signaling and oligodendroglial abnormalities in the temporal cortex from patients with major depressive disorder. Mol Psychiatry 10: 309-

Begemann M, Grube S, Papiol S, Malzahn D, Krampe H, Ribbe K, Friedrichs H, Radyushkin KA, El-Kordi A Benseler F, et al (2010) Modification of cognitive performance in schizophrenia by complexin 2 gene polymorphisms. Arch Gen Psychiatry 67: 879-888

Bercik P, Verdu EF, Foster JA, Macri J, Potter M, Huang X, Malinowski P, Jackson W, Blennerhassett P, Neufeld KA, et al (2010) Chronic gastrointestinal inflammation induces anxiety-like behavior and alters central nervous system biochemistry in mice. Gastroenterology 139: 2102-2112 e2101

Berrettini WH (2000) Are schizophrenic and bipolar disorders related? A review of family and molecular studies. Biol Psychiatry 48: 531-

Bifulco M, Laezza C, Stingo S, Wolff J (2002) 2', 3'-Cyclic nucleotide $3^{\prime}$ phosphodiesterase: a membrane-bound, microtubule-associated protein and membrane anchor for tubulin. Proc Natl Acad Sci USA 99: 18071812

Braun PE, Lee J, Gravel M (2004) 2',3'-cyclic nucleotide 3'-phosphodiesterase: structure, biology, and function. In: Myelin Biology and Disorders, Lazzarin RA (ed), San Diego: Elsevier Academic Press pp 499-522.

Brooks SP, Dunnett SB (2009) Tests to assess motor phenotype in mice: a user's guide. Nat Rev Neurosci 10: 519-529

Chaperon F, Thiebot MH (1999) Behavioral effects of cannabinoid agents in animals. Crit Rev Neurobiol 13: 243-281

Che R, Tang W, Zhang J, Wei Z, Zhang Z, Huang K, Zhao X, Gao J, Zhou G, Huang $P$, et al (2009) No relationship between $2^{\prime}, 3^{\prime}$-cyclic nucleotide $3^{\prime}$ phosphodiesterase and schizophrenia in the Chinese Han population: an expression study and meta-analysis. BMC Med Genet 10: 31

Chen EY, Shapleske J, Luque R, McKenna PJ, Hodges JR, Calloway SP, Hymas NF, Dening TR, Berrios GE (1995) The Cambridge Neurological Inventory: a clinical instrument for assessment of soft neurological signs in psychiatric patients. Psychiatry Res 56: 183-204

Cryan JF, Mombereau C, Vassout A (2005) The tail suspension test as a model for assessing antidepressant activity: review of pharmacological and genetic studies in mice. Neurosci Biobehav Rev 29: 571-625

Davis KL, Stewart DG, Friedman JI, Buchsbaum M, Harvey PD, Hof PR, Buxbaum J, Haroutunian V (2003) White matter changes in schizophrenia: evidence for myelin-related dysfunction. Arch Gen Psychiatry 60: 443456

De Angelis DA, Braun PE (1996) 2',3'-Cyclic nucleotide 3'-phosphodiesterase binds to actin-based cytoskeletal elements in an isoprenylationindependent manner. J Neurochem 67: 943-951

Derogatis LR, Melisaratos N (1983) The Brief Symptom Inventory: an introductory report. Psychol Med 13: 595-605

Dumser M, Bauer J, Lassmann H, Berger J, Forss-Petter S (2007) Lack of adrenoleukodystrophy protein enhances oligodendrocyte disturbance and microglia activation in mice with combined Abcd1/Mag deficiency. Acta Neuropathol 114: 573-586
Edgar IM, McLaughlin M, Werner HB, McCulloch MC, Barrie JA, Brown A, Faichney AB, Snaidero N, Nave KA, Griffiths IR (2009) Early ultrastructura defects of axons and axon-glia junctions in mice lacking expression of Cnp 1 Clia 57: 1815-1824

Finn DA, Rutledge-Gorman MT, Crabbe JC (2003) Genetic animal models of anxiety. Neurogenetics 4: 109-135

Gardner A, Boles RC (2011) Beyond the serotonin hypothesis: mitochondria, inflammation and neurodegeneration in major depression and affective spectrum disorders. Prog Neuropsychopharmacol Biol Psychiatry 35: 730743

Gravel M, Peterson J, Yong VW, Kottis V, Trapp B Braun PE (1996)

Overexpression of $2^{\prime}, 3^{\prime}$-cyclic nucleotide $3^{\prime}$-phosphodiesterase in transgenic mice alters oligodendrocyte development and produces aberrant myelination. Mol Cell Neurosci 7: 453-466

Gravel M, Robert F, Kottis V, Gallouzi IE, Pelletier J Braun PE (2009) 2', $3^{\prime}$-Cyclic nucleotide 3 '-phosphodiesterase: a novel RNA-binding protein that inhibits protein synthesis. J Neurosci Res 87: 1069-1079

Heckers S, Tandon R, Bustillo J (2010) Catatonia in the DSM-shall we move o not? Schizophr Bull 36: 205-207

Hofer S, Frahm I (2006) Topography of the human corpus callosum revisitedcomprehensive fiber tractography using diffusion tensor magnetic resonance imaging. Neuroimage 32: 989-994

Hofer S, Karaus A, Frahm J (2010) Reconstruction and dissection of the entire human visual pathway using diffusion tensor MRI. Front Neuroanat 4: 15 Ip CW, Kroner A, Bendszus M, Leder C, Kobsar I, Fischer S, Wiendl H, Nave KA Martini R (2006) Immune cells contribute to myelin degeneration and axonopathic changes in mice overexpressing proteolipid protein in oligodendrocytes. J Neurosci 26: 8206-8216

Iwamoto K, Ueda I, Bundo M, Nakano Y, Kato T (2008) Effect of a functional single nucleotide polymorphism in the $2^{\prime}, 3^{\prime}$-cyclic nucleotide $3^{\prime}$ phosphodiesterase gene on the expression of oligodendrocyte-related genes in schizophrenia. Psychiatry Clin Neurosci 62: 103-108

Jung M, Sommer I, Schachner M, Nave KA (1996) Monoclonal antibody 010 defines a conformationally sensitive cell-surface epitope of proteolipid protein (PLP): evidence that PLP misfolding underlies dysmyelination in mutant mice. J Neurosci 16: 7920-7929

Kahlbaum K (1874) Die Katatonie oder das Spannungsirresein., Berlin: Hirschwald

Karaus AH S., Frahm J, (2009) Separation of Fiber Tracts within the Human Cingulum Bundle using Single-Shot STEAM DTI. Open Med Imaging J 3: 21-27

Kassmann CM, Lappe-Siefke C, Baes M, Brugger B, Mildner A, Werner HB, Natt O, Michaelis T, Prinz M, Frahm J, et al (2007) Axonal loss and neuroinflammation caused by peroxisome-deficient oligodendrocytes. Nat Genet 39: 969-976

Kay SR, Fiszbein A, Opler LA (1987) The positive and negative syndrome scale (PANSS) for schizophrenia. Schizophr Bull 13: 261-276

Kimchi-Sarfaty C, Oh JM, Kim IW, Sauna ZE, Calcagno AM, Ambudkar SV, Gottesman MM (2007) A "silent" polymorphism in the MDR1 gene changes substrate specificity. Science 315: 525-528

Kuschinsky K, Hornykiewicz O (1972) Morphine catalepsy in the rat: relation to striatal dopamine metabolism. Eur J Pharmacol 19: 119. 122

Laan W, Grobbee DE, Selten JP, Heijnen CJ, Kahn RS, Burger H (2010) Adjuvant aspirin therapy reduces symptoms of schizophrenia spectrum disorders: results from a randomized, double-blind, placebo-controlled trial. , Clin Psychiatry 71: 520-527

Lappe-Siefke C, Goebbels S, Gravel M, Nicksch E, Lee J, Braun PE, Griffiths IR Nave KA (2003) Disruption of Cnp1 uncouples oligodendroglia functions in axonal support and myelination. Nat Genet 33: 366374

Lee J, Gravel M, Zhang R, Thibault P, Braun PE (2005) Process outgrowth in oligodendrocytes is mediated by CNP, a novel microtubule assembly myelin protein. J Cell Biol 170: 661-673 
Martini R, Toyka KV (2004) Immune-mediated components of hereditary demyelinating neuropathies: lessons from animal models and patients. Lancet Neurol 3: 457-465

Miller KR, Streit WJ (2007) The effects of aging, injury and disease on microglial function: a case for cellular senescence. Neuron Glia Biol 3: 245 253

Mitkus SN, Hyde TM, Vakkalanka R, Kolachana B, Weinberger DR, Kleinman JE, Lipska BK (2008) Expression of oligodendrocyte-associated genes in dorsolateral prefrontal cortex of patients with schizophrenia. Schizophr Res 98: 129-138

Monji A, Kato T, Kanba S (2009) Cytokines and schizophrenia: Microglia hypothesis of schizophrenia. Psychiatry Clin Neurosci 63: 257265

Morris R (1984) Developments of a water-maze procedure for studying spatial learning in the rat. I Neurosci Methods 11: 47-60

Moy SS, Nadler JJ, Perez A, Barbaro RP, Johns JM, Magnuson TR, Piven J, Crawley JN (2004) Sociability and preference for social novelty in five inbred strains: an approach to assess autistic-like behavior in mice. Genes Brain Behav 3: 287-302

Muller N, Schwarz MJ (2008) A psychoneuroimmunological perspective to Emil Kraepelins dichotomy: schizophrenia and major depression as inflammatory CNS disorders. Eur Arch Psychiatry Clin Neurosci 258: 97-106

Muller N, Krause D, Dehning S, Musil R, Schennach-Wolff R, Obermeier M, Moller HJ, Klauss V, Schwarz MJ, Riedel M (2010) Celecoxib treatment in an early stage of schizophrenia: results of a randomized, double-blind, placebo-controlled trial of celecoxib augmentation of amisulpride treatment. Schizophr Res 121: 118-124

Nielsen KB, Sorensen S, Cartegni L, Corydon TJ, Doktor TK, Schroeder LD, Reinert LS, Elpeleg O, Krainer AR, Gregersen N, et al (2007) Seemingly neutral polymorphic variants may confer immunity to splicing-inactivating mutations: a synonymous SNP in exon 5 of MCAD protects from deleterious mutations in a flanking exonic splicing enhancer. Am J Hum Genet 80: 416432

Northoff G, Kotter R, Baumgart F, Danos P, Boeker H, Kaulisch T, Schlagenhauf F, Walter H, Heinzel A, Witzel T, et al (2004) Orbitofrontal cortical dysfunction in akinetic catatonia: a functional magnetic resonance imaging study during negative emotional stimulation. Schizophr Bull 30: 405-427

Norton WT, Poduslo SE (1973) Myelination in rat brain: method of myelin isolation. J Neurochem 21: 749-757

O'Donovan MC, Craddock N, Norton N, Williams H, Peirce T, Moskvina V, Nikolov I, Hamshere M, Carroll L, Georgieva L, et al (2008) Identification of loci associated with schizophrenia by genome-wide association and followup. Nat Genet 40: 1053-1055

Peirce TR, Bray NJ, Williams NM, Norton N, Moskvina V, Preece A, Haroutunian V, Buxbaum JD, Owen MJ, O'Donovan MC (2006) Convergent evidence for $2^{\prime}, 3^{\prime}$-cyclic nucleotide $3^{\prime}$-phosphodiesterase as a possible susceptibility gene for schizophrenia. Arch Gen Psychiatry 63: 18-24

Radyushkin K, Hammerschmidt K, Boretius S, Varoqueaux F, El-Kordi A,

Ronnenberg A, Winter D, Frahm J, Fischer J, Brose N, et al (2009) Neuroligin3-deficient mice: model of a monogenic heritable form of autism with an olfactory deficit. Genes Brain Behav 8: 416-425

Radyushkin K, El-Kordi A, Boretius S, Castaneda S, Ronnenberg A, Reim K, Bickeboller H, Frahm J, Brose N, Ehrenreich $\mathrm{H}$ (2010) Complexin2 null mutation requires a 'second hit' for induction of phenotypic changes relevant to schizophrenia. Genes Brain Behav 9: 592-602

Ribbe K, Friedrichs H, Begemann M, Grube S, Papiol S, Kastner A, Gerchen MF, Ackermann V, Tarami A, Treitz A, et al (2010) The cross-sectional GRAS sample: a comprehensive phenotypical data collection of schizophrenic patients. BMC Psychiatry 10: 91

Scherer SS, Braun PE, Grinspan J, Collarini E, Wang DY, Kamholz J (1994) Differential regulation of the $2^{\prime}, 3^{\prime}$-cyclic nucleotide $3^{\prime}$-phosphodiesterase gene during oligodendrocyte development. Neuron 12: 1363-1375

Schnieder TP, Dwork A] (2011) Searching for neuropathology: gliosis in schizophrenia. Biol Psychiatry 69: 134-139

Sham PC, Jones P, Russell A, Gilvarry K, Bebbington P, Lewis S, Toone B, Murray $R$ (1994) Age at onset, sex, and familial psychiatric morbidity in schizophrenia. Camberwell Collaborative Psychosis Study. Br J Psychiatry 165: 466-473

Smith KJ, Lassmann $\mathrm{H}$ (2002) The role of nitric oxide in multiple sclerosis. Lancet Neurol 1: 232-241

Sparkman NL, Johnson RW (2008) Neuroinflammation associated with aging sensitizes the brain to the effects of infection or stress.

Neuroimmunomodulation 15: 323-330

Starkstein SE, Petracca G, Teson A, Chemerinski E, Merello M, Migliorelli R, Leiguarda R (1996) Catatonia in depression: prevalence, clinical correlates, and validation of a scale. J Neurol Neurosurg Psychiatry 60: 326-332

Stone EA, Lin Y (2011) Open-space forced swim model of depression for mice. Curr Protoc Neurosci 9: 36

Streit WJ (2006) Microglial senescence: does the brain's immune system have an expiration date? Trends Neurosci 29: 506-510

Tkachev D, Mimmack ML, Ryan MM, Wayland M, Freeman T, Jones PB, Starkey M, Webster MJ, Yolken RH, Bahn S (2003) Oligodendrocyte dysfunction in schizophrenia and bipolar disorder. Lancet 362: 798-805

Valles V, Van Os J, Guillamat R, Gutierrez B, Campillo M, Gento P, Fananas L (2000) Increased morbid risk for schizophrenia in families of in-patients with bipolar illness. Schizophr Res 42: 83-90

Yin X, Peterson J, Gravel M, Braun PE, Trapp BD (1997) CNP overexpression induces aberrant oligodendrocyte membranes and inhibits MBP accumulation and myelin compaction. J Neurosci Res 50: 238-247

Yu WP, Collarini EJ, Pringle NP, Richardson WD (1994) Embryonic expression of myelin genes: evidence for a focal source of oligodendrocyte precursors in the ventricular zone of the neural tube. Neuron 12: 1353-1362 


\subsection{Co-authorship publication 3}

\section{ORIGINAL ARTICLE}

\section{Neuropsychiatric disease relevance of circulating anti-NMDA receptor autoantibodies depends on blood-brain barrier integrity}

C Hammer ${ }^{1}$, B Stepniak ${ }^{1}$, A Schneider ${ }^{2,3,4}$, S Papiol ${ }^{1,3}$, M Tantra ${ }^{1,3}$, M Begemann ${ }^{1}$, A-L Sirén ${ }^{5}$, LA Pardo ${ }^{6}$, S Sperling ${ }^{1}$, S Mohd Jofrry ${ }^{1}$,

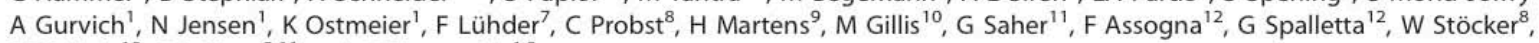
TF Schulz ${ }^{10}, \mathrm{~K}-\mathrm{A} \mathrm{Nave} \mathrm{N}^{3,11}$ and $\mathrm{H}$ Ehrenreich ${ }^{1,3}$

In 2007, a multifaceted syndrome, associated with anti-NMDA receptor autoantibodies (NMDAR-AB) of immunoglobulin-G isotype, has been described, which variably consists of psychosis, epilepsy, cognitive decline and extrapyramidal symptoms. Prevalence and significance of NMDAR-AB in complex neuropsychiatric disease versus health, however, have remained unclear. We tested sera of 2817 subjects ( 1325 healthy, 1081 schizophrenic, 263 Parkinson and 148 affective-disorder subjects) for presence of NMDAR-AB, conducted a genome-wide genetic association study, comparing $A B$ carriers versus non-carriers, and assessed their influenza $A B$ status. For mechanistic insight and documentation of $A B$ functionality, in vivo experiments involving mice with deficient bloodbrain barrier (ApoE ${ }^{-1-}$ ) and in vitro endocytosis assays in primary cortical neurons were performed. In 10.5\% of subjects, NMDAR$A B$ (NR1 subunit) of any immunoglobulin isotype were detected, with no difference in seroprevalence, titer or in vitro functionality between patients and healthy controls. Administration of extracted human serum to mice influenced basal and MK-801-induced activity in the open field only in $\mathrm{ApoE}^{-1-}$ mice injected with NMDAR-AB-positive serum but not in respective controls. Seropositive schizophrenic patients with a history of neurotrauma or birth complications, indicating an at least temporarily compromised bloodbrain barrier, had more neurological abnormalities than seronegative patients with comparable history. A common genetic variant (rs524991, $P=6.15 E-08)$ as well as past influenza $A(P=0.024)$ or $B(P=0.006)$ infection were identified as predisposing factors for NMDAR-AB seropositivity. The $>10 \%$ overall seroprevalence of NMDAR-AB of both healthy individuals and patients is unexpectedly high. Clinical significance, however, apparently depends on association with past or present perturbations of blood-brain barrier function.

Molecular Psychiatry advance online publication, 3 September 2013; doi:10.1038/mp.2013.110

Keywords: APOE; ApoE null mutant mice; autoimmunity; GWAS; influenza; NFIA

\section{INTRODUCTION}

$\mathrm{N}$-methyl-D-aspartate receptors (NMDAR) are glutamate-gated ion channels, abundantly expressed in mammalian brain. ${ }^{1}$ They form heteromers of NR1, NR2 and NR3 subunits, and are pivotal in regulating synapse function. ${ }^{2}$ In schizophrenia, NMDAR hypofunction has been hypothesized due to induction of psychotic symptoms by antagonists. ${ }^{3}$ In 2007, Dalmau et al. ${ }^{4,5}$ described a paraneoplastic syndrome, based on 12 women with ovarian teratoma, carrying IgG autoantibodies (AB) against the NMDAR NR1/2 subunits. The syndrome, termed 'anti-NMDAR encephalitis', variably consisted of psychosis, memory deficits, seizures, dyskinesia, decreased consciousness and autonomic instability. Since its initial description, a flood of publications appeared. The search for anti-NR1 lgG AB in small samples $(N=46-80)$ of schizophrenic patients yielded discordant results. ${ }^{6-8}$ Recently, $>400$ previously collected cases of anti-NMDAR encephalitis have been reviewed, most without associated tumor. ${ }^{9}$ Similarly, immunomodulatory treatment outcomes of these and around 100 more cases have been summarized. ${ }^{10}$ As a syndromepertinent pathophysiological mechanism, an AB-induced decrease of NMDAR-mediated currents, due to enhanced receptor internalization and thus reduced surface expression, has been suggested. ${ }^{11}$

Few studies explored a role of other classes of immunoglobulins (lg) in an NMDAR-AB syndrome. In individuals with slow cognitive impairment, anti-NR1 IgA AB were found, which affected synaptic protein expression and decreased NMDAR-mediated currents. ${ }^{12}$ Anti-NR1 IgM AB were described in a patient with bipolar disorder ${ }^{13}$ and in patients with herpes simplex encephalitis. ${ }^{14}$ In the largest study so far, investigating $\lg G$, IgA and $\lg M$, Steiner et $a l^{15}$ reported a higher prevalence of $A B$ of all isotypes in 121 schizophrenic patients, compared with healthy controls or patients suffering from affective disorders. Apart from tumors, no sound information is available yet on putative susceptibility factors for the development of anti-NR1 AB.

The present study was designed to (1) systematically screen in an unbiased fashion a large number $(N=2817)$ of healthy

${ }^{1}$ Clinical Neuroscience, Max Planck Institute of Experimental Medicine, Göttingen, Germany; ${ }^{2}$ Department of Psychiatry \& Psychotherapy, University Medicine Göttingen, Göttingen, Germany; ${ }^{3}$ DFG Research Center Nanoscale Microscopy and Molecular Physiology of the Brain (CNMPB), Göttingen, Germany; ${ }^{4}$ German Center for Neurodegenerative Diseases (DZNE), Göttingen, Germany: ${ }^{5}$ Department of Neurosurgery, University Clinic of Würzburg, Würzburg, Germany, ${ }^{6}$ Department of Molecular Biology of Neuronal Signals, Max Planck Institute of Experimental Medicine, Göttingen, Germany; 'Department of Neuroimmunology, Institute for Multiple Sclerosis Research and Hertie Foundation University Medicine Göttingen, Göttingen, Germany; ${ }^{8}$ Institute for Experimental Immunology, affiliated to Euroimmun, Lübeck, Germany; ${ }^{9}$ Synaptic Systems GmbH, Göttingen, Germany; ${ }^{10}$ Institute of Virology, Hannover Medical School, Hannover, Germany; ${ }^{11}$ Department of Neurogenetics, Max Planck Institute of Experimental Medicine, Göttingen, Germany and ${ }^{12}$ Department of Clinical and Behavioral Neurology, IRCCS Santa Lucia Foundation, Rome, Italy. Correspondence: Professor $\mathrm{H}$ Ehrenreich, Clinical Neuroscience, Max Planck Institute of Experimental Medicine, Göttingen 37075, Germany.

E-mail: ehrenreich@em.mpg.de

Received 22 May 2013; revised 19 July 2013; accepted 22 July 2013 
individuals and subjects suffering from schizophrenia or other brain diseases for presence of NMDAR-AB of $\lg G$, IgA or $\lg M$ isotype; (2) specifically address the question of why healthy $A B$ carriers might remain healthy, by extending this work to experiments in vivo and in vitro; (3) search for genetic and environmental factors predisposing to NMDAR-AB formation.

\section{MATERIALS AND METHODS}

\section{Participants}

Subject data were collected in accordance with ethical guidelines and the Declaration of Helsinki. Sample selection was unbiased, that is sera collection was concluded before analysis of NMDAR-AB was planned. Schizophrenic patients $(N=1081)$ were recruited from $2005-2011$ at 23 German sites in the GRAS (Göttingen Research Association for Schizophrenia) study. Patients fulfilling DSM-IV criteria for schizophrenia (81.5\%) or schizoaffective disorder $(18.5 \%)$ were included regardless of disease stage. $^{16,17}$ Healthy GRAS controls were anonymized blood donors ( $N=1272$; Transfusion Medicine, Göttingen). Health was ensured by predonation screening (questionnaires, interviews, hemoglobin, blood pressure, pulse, temperature). Patients with affective disorders $(N=148)$ were also included (ongoing GRAS extension). Parkinson patients $(N=263)$ and respective controls $(N=53)$ were recruited from 2010-2011 in Italy (Rome area). Of the GRAS patients, three volunteers carrying high titers of anti-NR1 IgG, IgA, or IgM, and three seronegative controls agreed to blood donation for mouse experiments (Supplementary Table S1, Supplementary Appendix).

\section{Phenotypical analyses}

On all schizophrenic (GRAS) patients, extensive phenotypical characterization was performed as referenced previously. ${ }^{16,17}$ Frequency and duration of prodrome, age at first psychotic episode, positive and negative syndrome scale (PANSS) scores, chlorpromazine equivalents, neurological symptoms (CNl; Cambridge Neurological Inventory) including fine motor skills (MacQuarrie dotting/tapping), current cognitive functioning (composite score comprising reasoning, executive function, verbal learning and memory), premorbid intelligence and global assessment of functioning (GAF) were employed as disease characteristics. As factors affecting blood-brain barrier (BBB) integrity, past neurotrauma (all severity levels) and birth complications (all pre- and perinatal complications) were carefully and comprehensively assessed. The final judgment regarding experience of birth complications or neurotrauma in the schizophrenic (GRAS) patient cohort was derived from a number of different sources. First, the information from semi-structured interviews about birth and neurotrauma history of each patient was used. To verify the data or increase the amount of detailed information, all discharge letters of each single patient were screened. In the case of neurotrauma, other semistructured interviews on critical life events, suicidality and aggressive behavior toward others were used to explore whether patients had experienced serious accidents (including brain trauma) or committed suicide attempts that included, for example, falls or jumps from great heights or had been involved in serious fights leading to head injuries. Finally, information from the physical exam of each patient was included to check whether any scars on the head or neck were found indicative of an injury to the head. After collecting all the data, each patient was dichotomously (yes/no) classified as having or not having experienced a neurotrauma or birth complication. In case of contradictory information, the treating physician and even the obstetric hospital were contacted, and in case of still missing information or a high level of uncertainty, patients were excluded from the analysis.

\section{Serological analyses}

Commercially available recombinant immunofluorescence tests (Euroimmun, Lübeck, Germany), standard procedures for clinical diagnosis (100\% sensitivity and $100 \%$ specificity), were used to detect NMDAR-AB, based on HEK293 cells transfected with NR1 or NR1/NR2b NMDAR-subunits., ${ }^{5,18}$ Seropositivity was assessed by two researchers independently. Titers were double-determined in two laboratories (MPI, Euroimmun) by identifying the maximum dilution at which specific fluorescence was still visible. Few samples with discrepant results were re-analyzed, leading to full concordance. The presence of IgG $A B$ against influenza $A$ and $B$ virus was determined by ELISA (Novagnost-InfluenzaA-lgG, NovagnostInfluenzaB-lgG, Siemens Healthcare Diagnostics GmbH, Eschborn,
Germany), automatically processed on BEPIII (Siemens Healthcare Diagnostics $\mathrm{GmbH}$ ), and interpreted (manufacturer's instructions) as positive, negative or borderline (the latter negative for statistics).

\section{Immunoglobulin purification}

Ammonium sulfate precipitation of a serum fraction containing immunoglobulins (Ig) and dialysis was carried out as described. ${ }^{19} \operatorname{lgG}$, $\lg A$, or $\lg M$ were quantified by immunodiffusion using NOR partigen immunoplates (Siemens Medical Solutions, Marburg, Germany).

\section{Mouse experiments}

Experiments were approved by the local Animal Protection Committee. Male $\mathrm{C} 57 \mathrm{BI} / 6 \mathrm{~N} \mathrm{ApoE}^{-/-20}$ and wild-type (WT) mice, aged $12-16$ weeks, were used (housed at 4-5 per cage, $12 \mathrm{~h}$ light/dark cycle, food/water ad libitum). Groups (4-6 each) received either extracted Ig fractions from NMDAR-AB seropositive (IgG, $\lg A$, or $\lg M$ ) or seronegative individuals (information on titer/concentration in Supplementary Table S1 and Supplementary Appendix). Daily intravenous (tail) injections ( $150 \mu \mathrm{l}$ each) were performed on 3 consecutive days. Examiners were not aware of group assignment ('blinded'). Spontaneous activity in open field ( $8 \mathrm{~min})$ was tested in all mice 3-4 days before the first injection (initial group matching). One day after the last injection, spontaneous activity $(8 \mathrm{~min}$ ) was again measured, followed by intraperitoneal injection $(0.3 \mu \mathrm{g}$ per $10 \mu \mathrm{l}$ per gram of body weight) of the non-competitive NMDAR antagonist MK801 (Dizocilpine; [5R,10S]-(+)-5-methyl-10,11-dihydro-5H-dibenzo[a,d]cy-

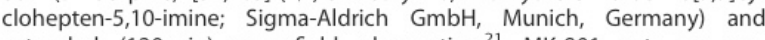
extended $(120 \mathrm{~min})$ open-field observation. ${ }^{21}$ MK-801 acts as usedependent ion-channel blocker.

Endocytosis assays and quantification

Primary cortical neurons prepared from mouse embryos (E16) were cultured on poly-L-lysine-coated glass-coverslips in MEM + B27 (Invitrogen Karlsruhe, Germany) for 14 days. Glass coverslips were washed with cold Hank's balanced salt solution (HBSS), and incubated $\left(20 \mathrm{~min}, 4^{\circ} \mathrm{C}\right)$ with Ig extracts containing either NMDAR-AB of $\operatorname{lgG}$, IgA or $\lg M$ isoforms or seronegative Ig extracts $(1: 100)$ in HBSS. The examiner was unaware of the nature of the extract ('blinded'). Unbound AB was removed (3 HBSS washes) before placing cells in pre-warmed $\left(37^{\circ} \mathrm{C}\right)$ growth medium for $15 \mathrm{~min}$ to allow internalization. After medium wash-off (cold HBSS), remaining surface NMDAR were labeled with anti-mouse NR1-AB (Abcam, Cambridge, UK) for $15 \mathrm{~min}$ on ice. After cold HBSS wash, surface-bound NR1-AB was labeled $\left(4^{\circ} \mathrm{C}, 15 \mathrm{~min}\right)$ with Alexa-488-coupled 2 nd AB (antimouse IgG; Invitrogen, Karlsruhe, Germany). After wash-off (HBSS; $4^{\circ} \mathrm{C}$ ) of unbound $A B$, cells were fixed in $4 \%$ paraformaldehyde. For quantification, confocal images of cell surface staining were taken with identical acquisition parameter on SP2 LSM (Leica, Wetzlar, Germany). Signal intensity was quantified with ImageJ, and ratio of intensity per cell surface area calculated.

\section{Genetic analyses}

A semi-custom Axiom myDesign genotyping array (Affymetrix, Santa Clara, CA, USA) was used. For description of array-specifications, quality controls and genome-wide genetic association study (GWAS), see Supplementary Appendix.

\section{Statistical analysis}

$P$-values $<0.05$ were considered significant. Data in figures are expressed as mean \pm s.e.m., in tables as mean \pm s.d.

Mouse experiments. Data were compared by analysis of variance, followed by post-hoc tests where appropriate using Prism5 (GraphPad-Software Inc., La Jolla, CA, USA) or SPSS (SPSS-Statistics 17.0, IBM-Deutschland GmbH, Munich, Germany). Greenhouse-Geisser correction was applied on violation of sphericity.

Human data. Group differences in categorical and continuous variables were assessed using Chi-square or Mann-Whitney $U$ tests. A generalized linear model was employed upon covariate inclusion. In case of normal distribution of continuous variables, $T$-tests were performed. To assess main effects and interactions between neurotrauma/birth complications and Ig-positivity regarding CNI scores, two-way independent ANOVA was 
Neuropsychiatric disease relevance of circulating NMDAR-AB

C Hammer et al

Table 1. Prevalence of anti-NR1 AB in patients and controls

\begin{tabular}{|c|c|c|c|c|c|c|c|c|}
\hline \multirow[b]{2}{*}{ Study group } & \multicolumn{8}{|c|}{ Anti-NR1 seropositivity-N (\%) } \\
\hline & Any & $\lg G$ & $\lg A$ & $\lg M$ & $\lg G+\lg A$ & $\lg G+\lg M$ & $\lg A+\lg M$ & $\lg G+\lg A+\lg M$ \\
\hline GRAS $^{\text {a }}$ patients $(N=1081)$ & $93(8.6)$ & $7(0.7)$ & $56(5.2)$ & $46(4.3)$ & $0(0)$ & $0(0)$ & $16(1.5)$ & $0(0)$ \\
\hline Affective-disorder patients $(N=148)$ & $24(16.2)$ & $5(3.4)$ & $15(10.1)$ & $7(4.7)$ & $1(0.7)$ & $0(0)$ & $2(1.4)$ & $0(0)$ \\
\hline GRAS $^{\text {a }}$ controls $(N=1272)$ & $137(10.8)$ & $5(0.4)$ & $75(5.9)$ & $80(6.3)$ & $2(0.2)$ & $3(0.2)$ & $19(1.5)$ & $1(0.1)$ \\
\hline Parkinson patients $(N=263)$ & 35 (13.3) & $1(0.4)$ & $17(6.5)$ & $25(9.5)$ & $1(0.4)$ & $1(0.4)$ & $7(2.7)$ & $1(0.4)$ \\
\hline Parkinson controls $(N=53)$ & $6(11.3)$ & $0(0)$ & $3(5.7)$ & $3(5.7)$ & $0(0)$ & $0(0)$ & $0(0)$ & $0(0)$ \\
\hline Total $(N=2817)$ & $295(10.5)$ & $18(0.6)$ & $166(5.9)$ & $161(5.7)$ & $4(0.1)$ & $4(0.1)$ & $44(1.6)$ & $2(0.1)$ \\
\hline
\end{tabular}

aRAS patients are schizophrenic individuals of the GRAS data collection (Göttingen Research Association for Schizophrenia). GRAS controls are the respective healthy control collective (see also Materials and methods).

conducted. Corrected values reflect linear regression-based residuals when age, chlorpromazine and PANSS negative scores were independent variables. PLINK (v1.07) was used to test association between singlenucleotide polymorphisms (SNPs) and anti-NR1 serological status (allelic test) and deviations from Hardy-Weinberg equilibrium. ${ }^{22}$ Principal components were generated using EIGENSTRAT (http:// genetics.med.harvard.edu/reich/Reich Lab/Software.html). Human leukocyte antigen (HLA) types were imputed for seven HLA genes using HiBag0.9.1 at four-digit resolution, based on a pre-fit European ancestry model (http://cran.r-project.org/web/packages/HIBAG/index.html). P-values were multiple-testing corrected (Bonferroni) where indicated, but are displayed uncorrected.

\section{RESULTS}

NMDAR-AB seroprevalence in 2817 individuals

$A B$ of all here analyzed isotypes (IgG, $\lg A$ and $\lg M)$, directed against the NMDAR-NR1 subunit, were identified in $10.5 \%$ of subjects (Table 1). Importantly, seroprevalence did not differ between schizophrenic (GRAS) patients (8.6\%) and GRAS controls $(10.8 \%, P=0.078)$. An apparently higher incidence in affectivedisorder patients $(16.2 \%)$ is explained by a higher mean age. In fact, seroprevalence increases with age (Supplementary Figure S1, Supplementary Table S2, Supplementary Appendix) and is higher in male than female subjects (Supplementary Table S3, Supplementary Appendix, $11.53 \%$ versus $8.68 \%, P=0.017)$. Seropositivity between Parkinson patients (13.3\%) and respective controls did not differ $(11.3 \%, P=0.694)$.

Seroprevalence and titer distribution of NMDAR-AB Ig isotypes Considering each Ig class separately, again no differences in seroprevalence among groups arose (Table 1). Occurrence of IgG anti-NR1 was infrequent (0.6\% in total) compared with IgA (5.9\%) or $\lg M(5.7 \%)$. A combination of $\lg A / \lg M A B$ was present in $1.6 \%$, combinations including IgG in only $0.1 \%$ each. $A B$ exclusively against the NR1/NR2b heterodimer, that is without reactivity against NR1 alone, were not identified. Titer distributions in patient and control groups as possible explanation of NMDAR-AB pathology did not differ (Supplementary Table S4, Supplementary Figure S2, Supplementary Appendix).

NMDAR-AB functionality in a neuronal endocytosis assay We next wondered whether $A B$ from controls and patients differ in functionality. Extracts from seropositive subjects, independently of isotype or disease state, resulted in increased endocytosis, compared with seronegative extracts (Figure 1a, Supplementary Table S1, Supplementary Appendix).

Relevance of BBB integrity for NMDAR-AB effects in mice Having comparable serological (\% seropositivity, Ig-isotype, titer distribution) and functional results in controls and patients, we asked why healthy $A B$ carriers remain healthy. We hypothesized that a compromised BBB might decide on the pathophysiological significance of NMDAR-AB. To approach this hypothesis experimentally, we employed $\mathrm{ApoE}^{-}$mice $^{20}$ (with known BBB leakage $)^{23-25}$ versus WT. Intravenous injection of purified lg fractions from NMDAR-AB seropositive (IgM, $\lg G, \lg A$ ) subjects led to alterations in spontaneous open-field activity and the response to MK-801 exclusively in $\mathrm{ApoE}^{-/-}$mice. Trends were comparable in groups receiving $\lg \mathrm{M}, \lg \mathrm{g}$ or $\lg \mathrm{A}$ extracts, resulting in significant differences on pooling (Figures $1 \mathrm{~b}$ and $\mathrm{c}$, Supplementary Figure S3, Supplementary Appendix).

Translating experimental BBB findings to schizophrenic (GRAS) patients

Overall, schizophrenic anti-NR1 carriers and non-carriers do not differ with respect to disease phenotypes, covering the symptom clusters of anti-NMDAR encephalitis (Table 2). Also, occurrence and duration of prodromal phase and age of disease onset are similar between the two groups, arguing against a sudden/ atypical syndrome start in $A B$ carriers (Table 2). Following our BBB hypothesis, we compared individuals with birth complications or past brain trauma-conditions known to provoke temporary or persistent (albeit often minor) BBB abnormalities. ${ }^{26,27}$ Indeed, also in humans, a clear impact, that is a more severe neurological phenotype, arises from the combination of compromised BBB function and circulating NMDAR-AB (Figure 1d).

Identification of first genetic susceptibility factors GWAS have been successful in identifying associations between genomic variants and autoimmune disorders, such as rheumatoid arthritis or systemic lupus erythematosus. ${ }^{28}$ We performed GWAS to spot SNPs potentially predisposing to formation of NMDAR-AB (Supplementary Appendix). We identified a genomewide significant SNP, rs 524991 (A/G, $P=6.15 \mathrm{E}-08$; Bonferroni threshold $P=8.62 \mathrm{E}-08$ ), with an odds ratio (OR) of 2.22 (95\% confidence interval $(\mathrm{Cl})=1.654-2.991$; Supplementary Figure S6, Supplementary Appendix). This variant with a minor allele frequency of $12.45 \%$ in seropositive versus $6.01 \%$ in seronegative individuals is located in an intergenic region on chromosome1 (Supplementary Figure S7, Supplementary Appendix). Its closest neighboring gene is nuclear factor $\mathrm{I} / \mathrm{A}$ (NFIA, $218.59 \mathrm{~kb}$ downstream), a transcription factor reported to mediate neuroprotective effects of NMDAR activation. ${ }^{29}$ Separate analysis of SNP rs524991 association with NMDAR$A B$ seropositivity (Table 3 ) showed a similar tendency for all study groups (except Parkinson) and no gender difference (Supplementary Table S8, Supplementary Appendix). Search for a predisposing role of HLA alleles for NMDAR-AB formation did not deliver hits, apart from a nominally significant association of HLA-A03 with seropositivity ( $P=0.01$; Supplementary Table S9, Supplementary Appendix). 

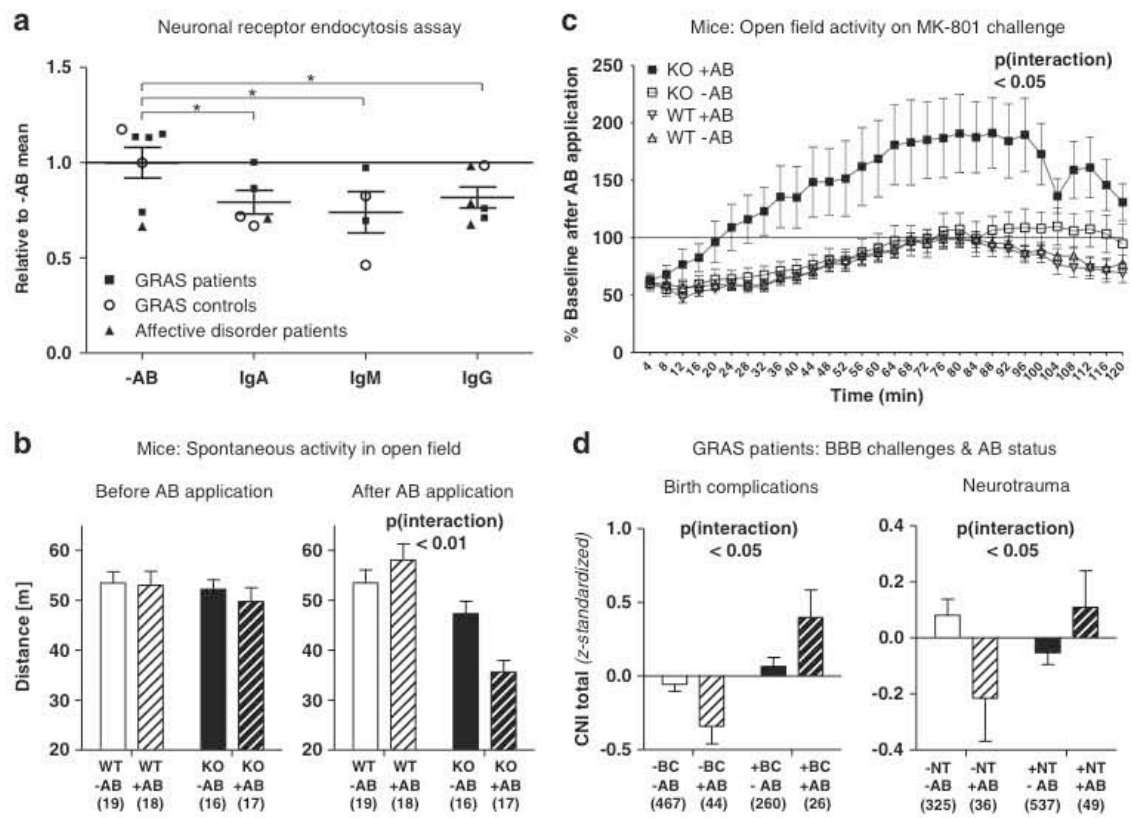

Figure 1. NMDAR-AB functionality and relevance of the blood-brain barrier. (a) Reduced $A B$ binding to primary cortical neurons indicates increased endocytosis of NMDAR after incubation with Ig extracts containing either NMDAR-AB of IgG, IgA or IgM isoforms (all $P<0.05$ ), or seronegative $\mathrm{lg}$ extracts (one-tailed T-tests). Mean values upon AB extracts (each tested in 1-3 independent experiments, dependent on serum availability) were normalized to the mean of the respective seronegative control extracts. (b) ApoE ${ }^{-1-}\left(\mathrm{KO}^{-}\right)$and WT mice do not differ in spontaneous activity in the open field before $A B$ injection. However, 1 day after the last of three daily injections with seropositive ( $+A B$ ) or seronegative $(-A B)$ Ig extracts, a decrease in spontaneous activity was evident exclusively in seropositive $A p o E^{-1}(K O+A B)$ mice: two-way ANOVA revealed a significant interaction effect of genotype and serotype $(F=8.96, P<0.01)$, as well as a significant main effect of genotype $(F=27.81, P<0.0001)$, but not of serotype. (c) Only ApoE ${ }^{-1-}(\mathrm{KO})$ mice with their known compromised BBB respond to intravenous NMDAR$A B$ extracts with a hypersensitive (psychosis-related) response in the open field to the NMDAR antagonist MK-801. Using a generalized linear model for repeated measures to evaluate results after MK- 801 treatment, we obtained significant main effects of time $(F=36.25, P<0.001)$, genotype $(F=9.54, P<0.01)$ and serotype $(F=4.85, P<0.05)$ as well as a significant genotype $\times$ serotype interaction effect $(F=5.75, P<0.05)$. (d) In the GRAS sample of schizophrenic patients, birth complications and history of neurotrauma as readouts for temporarily/persistently compromised BBB were examined in NMDAR-AB-positive versus negative subjects with respect to their impact on neurological symptom severity (CNI total score, $z$-standardized). Birth complications showed an interaction with serotype $(F=5.80, P<0.05)$ regarding $C N I$ and a main effect $(F=11.24, P=0.001)$. Likewise, past neurotrauma showed an interaction with serotype $(F=4.02, P<0.05)$. Group sizes are given as

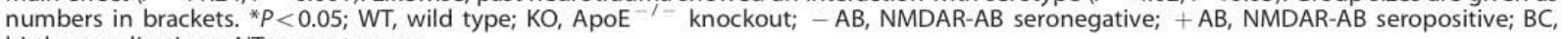
birth complications; NT, neurotrauma.

Identification of environmental susceptibility factors

As first risk factor for NMDAR-AB formation, the presence of a tumor, preferentially an ovarian teratoma was identified. ${ }^{4}$ Other predisposing factors have remained speculative. Infections have been suggested as triggers of $A B$ formation in autoimmune diseases, ${ }^{30}$ for example, Epstein-Barr virus in multiple sclerosis. ${ }^{31}$ We hypothesized that a similar role might be attributed to influenza for NMDAR-AB. Anti-NMDAR encephalitis was reported in a patient with influenza $\mathrm{H} 1 \mathrm{~N} 1$ infection and two subjects after respective vaccination. ${ }^{9}$ NMDAR-AB were described in pediatric cases of encephalitis lethargica, a condition - not unequivocallyassociated with influenza. ${ }^{32,33}$ Strikingly, we found an increased anti-NR1 $A B$ prevalence in individuals carrying anti-influenza $A$ $(P=0.024, \mathrm{OR}=1.366, \mathrm{Cl} 95 \%=1.042-1.790)$ and $\mathrm{B}(P=0.006$, $\mathrm{OR}=1.453, \mathrm{Cl} 95 \%=1.109-1.904) \mathrm{lgG}$ (Table 3). This association was present in males only (Supplementary Table S10, Supplementary Appendix).

\section{DISCUSSION}

The present study provides (1) the first large-scale systematic screen for presence of NMDAR-AB in serum of healthy and neuropsychiatrically diseased subjects. In $>2800$ unbiasedly selec ted individuals, $>10 \%$ seroprevalence of anti-NR1 AB, independent of group affiliation, was found. (2) From this unexpected observation, the fundamental question arose of why healthy $A B$ carriers have remained healthy, despite comparable distribution of $A B$ isotypes, titers and in vitro functionality. An experimental mouse model supports our central hypothesis, that is, the essential role of $\mathrm{BBB}$ integrity. Only in ApoE mutant mice, but not in respective controls, we find that human NMDAR-AB cause psychosis-related behavioral perturbation. ${ }^{21}$ The BBB role is further underlined by a hypothesis-driven outcome analysis of schizophrenic (GRAS) patients with history of birth complications or neurotrauma indicating past/present BBB insufficiency. (3) Ultimately, with a genome-wide significant marker, SNP rs524991, and an association of seropositivity with influenza $A B$ status, we provide genetic and environmental risk factors of NMDAR-AB formation.

The most remarkable finding of the present work is the high seroprevalence of NMDAR-AB in healthy individuals. Only one other study included a considerable number of healthy subjects in a screen of psychiatric patients but reported seropositivity for only 1 in 240 controls $(0.4 \%)$ and 2 in $108(<2 \%)$ affective-disorder patients. ${ }^{15}$ Seroprevalence in schizophrenic patients $(9.9 \%$ of 121$)$ 
Neuropsychiatric disease relevance of circulating NMDAR-AB C Hammer et al

\begin{tabular}{|c|c|c|c|c|}
\hline Patients and control groups & Total sample & Ig-positive individuals & Ig-negative individuals & P-value $\left(\chi^{2}, Z, T \text { value }\right)^{\mathrm{a}}$ \\
\hline Schizophrenic (GRAS) patients & $N=774-1081^{b}$ & $N=63-93^{b}$ & $N=711-988^{\mathrm{b}}$ & \\
\hline Age, years & $39.37 \pm 12.59(17-79)$ & $41.15 \pm 11.98(18-75)$ & $39.20 \pm 12.63(17-79)$ & $0.115(Z=-1.58)$ \\
\hline Gender, $N$ male (\%) & $723(66.9)$ & $68(73.1)$ & $655(66.3)$ & $0.181\left(\gamma^{2}=1.79\right)$ \\
\hline Prodrome, $N$ cases (\%) & $754(80.6)$ & $64(79.0)$ & $690(80.8)$ & $0.698\left(\gamma^{2}=0.15\right)$ \\
\hline Duration of prodrome, years & $2.81 \pm 3.57(0-28.2)$ & $2.64 \pm 3.01(0-13.0)$ & $2.83 \pm 3.62(0-28.2)$ & $0.853(Z=-0.19)$ \\
\hline Age at first episode, years & $25.88 \pm 8.90(5-68)$ & $25.85 \pm 8.98(12-51)$ & $25.89 \pm 8.90(5-68)$ & $0.890(Z=-0.11)$ \\
\hline PANSS positive score & $13.74 \pm 6.25(7-38)$ & $13.28 \pm 5.71(7-31)$ & $13.78 \pm 6.31(7-38)$ & $0.597(Z=0.60)$ \\
\hline PANSS negative score & $18.25 \pm 7.90(7-46)$ & $17.00 \pm 7.42(7-37)$ & $18.37 \pm 7.93(7-46)$ & $0.122(Z=-1.55)$ \\
\hline PANSS general score & $33.74 \pm 11.76(16-82)$ & $32.48 \pm 10.80(16-75)$ & $33.86 \pm 11.85(16-82)$ & $0.373(Z=-0.89)$ \\
\hline $\mathrm{CPZ}$ & $686.53 \pm 697.43(0-7375)$ & $628.04 \pm 537.82(0-2620)$ & $691.97 \pm 710.43(0-7375)$ & $0.580(Z=-0.55)$ \\
\hline $\mathrm{CNI}^{\mathrm{c}, \mathrm{d}}$ & $0.00 \pm 1.00(-2.71-3.07)$ & $-0.03 \pm 0.92(-2.07-2.11)$ & $0.00 \pm 1.01(-2.71-3.07)$ & $0.742(T=0.33)$ \\
\hline MacQuarrie dotting ${ }^{e}$ & $0.00 \pm 1.00(-3.61-3.22)$ & $0.14 \pm 1.14(-2.67-3.07)$ & $-0.01 \pm 0.98(-3.61-3.22)$ & $0.172(T=-1.37)$ \\
\hline MacQuarrie tapping ${ }^{e}$ & $0.00 \pm 1.00(-4.83-3.14)$ & $0.11 \pm 0.99(-2.00-3.10)$ & $-0.01 \pm 1.00(-4.83-3.14)$ & $0.261(T=-1.13)$ \\
\hline Cognitive composite score & $-0.02 \pm 0.84(-2.57-2.98)$ & $-0.01 \pm 0.89(-2.12-2.03)$ & $-0.02 \pm 0.84(-2.57-2.98)$ & $0.946(T=-0.07)$ \\
\hline Premorbid IQ (MWT-B ${ }^{f}$ ) & $25.67 \pm 6.36(4-42)$ & $26.64 \pm 6.28(6-36)$ & $25.57 \pm 6.36(4-42)$ & $0.093(Z=-1.68)$ \\
\hline GAF & $45.70 \pm 17.18(5-90)$ & $46.26 \pm 16.54(10-80)$ & $45.65 \pm 17.25(5-90)$ & $0.642(Z=-0.47)$ \\
\hline Neurotrauma, $N$ cases $(\%)$ & $648(62.4)$ & $55(62.6)$ & $593(59.8)$ & $0.592\left(\chi^{2}=0.29\right)$ \\
\hline Birth complications, $N$ cases (\%) & $307(39.7)$ & $27(42.9)$ & $280(39.4)$ & $0.589\left(\chi^{2}=0.29\right)$ \\
\hline Healthy (GRAS) controls & $N=1272$ & $N=137$ & $N=1135$ & \\
\hline Age, years & $37.43 \pm 13.24(18-69)$ & $40.90 \pm 12.17(19-68)$ & $37.01 \pm 13.31(18-69)$ & $<0.001(Z=-3.56)$ \\
\hline Gender, $N$ male $(\%)$ & $780(61.3)$ & $95(69.3)$ & $685(60.4)$ & $0.041\left(\chi^{2}=4.17\right)$ \\
\hline Affective-disorder patients & $N=148$ & $N=24$ & $N=124$ & \\
\hline Age, years & $49.70 \pm 15.49(20-92)$ & $47.38 \pm 11.87(25-76)$ & $50.15 \pm 16.09(20-92)$ & $0.314(Z=-1.01)$ \\
\hline Gender, $N$ male (\%) & $70(47.3)$ & $11(45.8)$ & $59(47.6)$ & $0.875\left(\chi^{2}=0.03\right)$ \\
\hline Parkinson patients & $N=253-263^{\mathrm{b}}$ & $N=33-35^{\mathrm{b}}$ & $N=220-228^{b}$ & \\
\hline Age, years & $66.04 \pm 10.08(36-86)$ & $69.06 \pm 8.33(45-81)$ & $65.59 \pm 10.26(36-86)$ & $0.055(Z=-1.92)$ \\
\hline Gender, $N$ male $(\%)$ & $175(66.5)$ & $28(80.0)$ & $147(64.5)$ & $0.070\left(\chi^{2}=3.29\right)$ \\
\hline Parkinson controls & $N=51-53^{b}$ & $N=6^{\mathrm{b}}$ & $N=45-47^{\mathrm{b}}$ & \\
\hline Age, years & $63.31 \pm 11.68(22-80)$ & $67.50 \pm 12.58(44-78)$ & $62.76 \pm 11.59(22-80)$ & $0.188(Z=-1.33)$ \\
\hline Gender, $N$ male (\%) & $21(39.6)$ & $2(33.3)$ & $19(40.4)$ & $0.738\left(\chi^{2}=0.11\right)$ \\
\hline
\end{tabular}

was comparable to our study. Reasons for this discrepancy are unclear but perhaps related to the smaller number of controls and their younger age. ${ }^{15}$ Importantly, analytical materials/methods of both studies were identical, schizophrenia patients show comparable seroprevalence, and the here randomly selected positive specimens for in vitro analyses all confirmed $A B$ functionality.

For exerting pathological effects, NMDAR-AB have to reach NMDAR in the brain. This brain presence may occur via (1) $A B$ transfer over a compromised BBB, which normally restricts large molecules from directly entering the brain in appreciable amounts (expected transfer over an intact BBB, for example, of lgG is only $1 / 500$, of $\operatorname{IgA} 1 / 600$, and of IgM $1 / 3000$ of the serum concentration); (2) slow accumulation of these large molecules due to reduced cerebrospinal fluid (CSF) flow, ${ }^{34}$ in which case, however, a retrograde CSF circulation would have to deliver the $A B$ back to brain tissue, or (3) intrathecal synthesis by B lymphocytes. ${ }^{34,35}$ Our seroprevalence data do not allow any conclusion on $A B$ production in brain. Therefore, healthy $A B$ carriers may differ at least in part from seropositive disease groups by lack of intrathecal $A B$ synthesis. Nevertheless, with in vivo experiments in mice and a hypothesis-driven human database screening, we underscore the likely critical role of an intact BBB as protective mechanism against circulating $A B$-mediated pathology in mouse and man.

Wild-type mice were not behaviorally affected after injection of human serum extract containing $\lg G, \lg A$ or $\lg M$ NMDAR-AB.
In contrast, $\mathrm{ApoE}^{-1-}$ mice showed differences in behavior on $A B$ injection, that is reduced spontaneous activity in the open field and hyperlocomotion following MK-801. These behavioral phenomena may be explained by the reported receptor internalization and hypofunction after hippocampal infusion of NMDAR-AB, ${ }^{11}$ as exactly opposite effects were described after NMDA application in rats. ${ }^{36}$ Stimulation of locomotion by the NMDAR antagonist MK-801 may be caused by hyperexcitability of limbic circuits through NMDAR blockade on inhibitory GABAergic neurons. ${ }^{37,38}$ This consequence of NMDA receptor inhibition would be amplified by NMDAR-AB. Similarly, increased motor cortex excitability in mice was provoked by NMDAR-AB injection into the prefrontal area. ${ }^{39}$

In the GRAS sample of schizophrenic individuals, well-documented history of birth complications and brain trauma were evaluated as proxy variables of past or present BBB impairment. ${ }^{26,27}$ Indeed, affected individuals show more severe neurological abnormalities when carrying NMDAR-AB. These findings strengthen the hypothesis of $B B B$ involvement in NMDAR-AB pathology, and-replication provided-may even justify recommendations of anti-NR1 serum screening in case of neurotrauma or other conditions with anticipated BBB dysfunction.

Importantly, we did not find any clinically relevant differences when comparing all schizophrenic NMDAR-AB carriers with all non-carriers. Perhaps with information on CSF (which we do not have in our large cohort), an expected $30 \%$ of individuals with 
$C$ Hammer et al

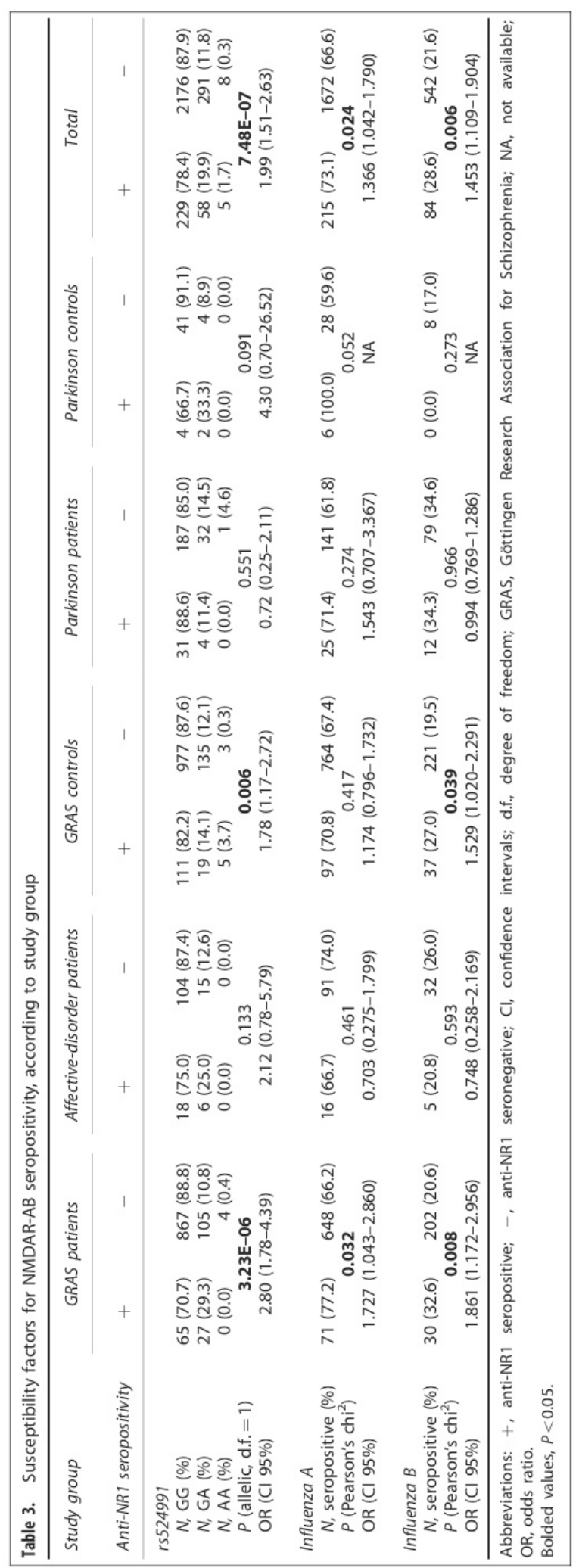

permanent barrier dysfunction ${ }^{40}$ could have been extracted and would have allowed us to uncover a clinically relevant difference between $A B$ carriers and non-carriers among them. Instead, we found a clinical difference between $A B$ carriers and non-carriers with past birth complication or neurotrauma as a proxy for at least temporary BBB disturbance. ${ }^{26}$ It is interesting to speculate that the reported $30 \%$ of schizophrenic patients with compromised barrier function ${ }^{40}$ and the post-trauma individuals recognized here might represent an (partly) overlapping subpopulation of schizophrenic subjects. Along these lines of thought, future studies may be initiated, analyzing CSF samples of a large number of schizophrenic patients for NMDAR-AB titers and determining the CSF-serum albumin quotient ${ }^{34}$ as marker of blood-CSF barrier (dys)function.

Our study is the first to investigate putative genetic susceptibility factors for the formation of anti-NR1 AB. A GWAS approach led to the identification of the genome-wide significant risk SNP rs524991. Further experiments providing mechanistic insight as well as replication analyses are warranted. By a hypothesis-driven approach, ${ }^{9,32,33}$ we uncovered an association of influenza $A$ or $B A B$ with anti-NR1 seropositivity, suggesting molecular mimicry. This phenomenon induces generation of $A B$ reacting both against pathogenic elements and autoantigens, ${ }^{30}$ and has a role in autoimmune diseases. ${ }^{41,42}$ As the influenza $A$ M2 channel and NMDAR share a common ligand, the antiviral compound amantadine, ${ }^{43}$ a putative structural homology might act as inducer of NMDAR-AB. The observed association was found in males only. Interestingly, males have a higher incidence of influenza, ${ }^{44}$ and male mice exert a more vigorous immune response on influenza infection. ${ }^{45}$ This gender disposition might also explain the higher anti-NR1 $A B$ prevalence in males.

In conclusion, our study draws an increasingly complex picture of NMDAR-AB pathology, with anti-NMDAR encephalitis possibly constituting the extreme end of a broad spectrum of mild to severe phenotypes associated with NMDAR autoimmunity. Beyond the NMDAR-AB studied here, loss of blood-brain barrier integrity may generally constitute a major risk factor for detrimental effects of peripheral $A B$ against central nervous system epitopes.

\section{CONFLICT OF INTEREST}

Dr Stöcker is a full-time employee of and holds stocks in Euroimmun AG. Dr Martens is a full-time employee of Synaptic Systems GmbH. All other authors declare no conflict of interest.

\section{ACKNOWLEDGEMENTS}

This work was supported by the Max Planck Society, the Max Planck Förderstiftung, and the DFG (CNMPB). Christian Hammer acknowledges grant support by the Daimler and Benz Foundation as well as by the Brain and Behavior Foundation. Suhaidah Mohd Jofrry, through support by the Faculty of Pharmacy, Universiti Teknologi MARA, Bandar Puncak Alam, Selangor, Malaysia, received a scholarship from the Ministry of Higher Education Malaysia. We are grateful to Sylvia Siefarth, Euroimmun, for excellent technical assistance.

\section{REFERENCES}

1 Li F, Tsien JZ. Memory and the NMDA receptors. N Engl J Med 2009; 361:302-303. 2 Lau CG, Zukin RS. NMDA receptor trafficking in synaptic plasticity and neuropsychiatric disorders. Nat Rev Neurosci 2007; 8: 413-426.

3 Homayoun H, Moghaddam B. NMDA receptor hypofunction produces opposite effects on prefrontal cortex interneurons and pyramidal neurons. J Neurosci 2007; 27: 11496-11500.

4 Dalmau J, Tuzun E, Wu HY, Masjuan J, Rossi JE, Voloschin A et al. Paraneoplastic anti-N-methyl-D-aspartate receptor encephalitis associated with ovarian teratoma. Ann Neurol 2007; 61: 25-36. 
5 Dalmau J, Gleichman AJ, Hughes EG, Rossi JE, Peng X, Lai M et al. Anti-NMDAreceptor encephalitis: case series and analysis of the effects of antibodies. Lancet Neurol 2008; 7: 1091-1098.

6 Masdeu JC, Gonzalez-Pinto A, Matute C, Ruiz De Azua S, Palomino A, De Leon J et al. Serum IgG antibodies against the NR1 subunit of the NMDA receptor not detected in schizophrenia. Am J Psychiatry 2012; 169: 1120-1121.

7 Zandi MS, Irani SR, Lang B, Waters P, Jones PB, McKenna P et al. Disease-relevant autoantibodies in first episode schizophrenia. J Neurol 2011; 258: 686-688.

8 Tsutsui K, Kanbayashi T, Tanaka K, Boku S, Ito W, Tokunaga J et al. Anti-NMDA receptor antibody detected in encephalitis, schizophrenia, and narcolepsy with psychotic features. BMC Psychiatry 2012; 12: 37.

9 Dalmau J, Lancaster E, Martinez-Hernandez E, Rosenfeld MR, Balice-Gordon R. Clinical experience and laboratory investigations in patients with anti-NMDAR encephalitis. Lancet Neurol 2011; 10: 63-74.

10 Titulaer MJ, McCracken L, Gabilondo I, Armangue T, Glaser C, lizuka T et al. Treatment and prognostic factors for long-term outcome in patients with antiNMDA receptor encephalitis: an observational cohort study. Lancet Neurol 2013; 12: $157-165$.

11 Hughes EG, Peng X, Gleichman AJ, Lai M, Zhou L, Tsou R et al. Cellular and synaptic mechanisms of anti-NMDA receptor encephalitis. J Neurosci 2010; 30: 5866-5875.

12 Pruss $\mathrm{H}$, Holtje M, Maier N, Gomez A, Buchert R, Harms L et al. IgA NMDA receptor antibodies are markers of synaptic immunity in slow cognitive impairment. Neurology 2012; 78: 1743-1753.

13 Choe CU, Karamatskos E, Schattling B, Leypoldt F, Liuzzi G, Gerloff C et al. A clinical and neurobiological case of IgM NMDA receptor antibody associated encephalitis mimicking bipolar disorder. Psychiatry Res 2012; 208: 194-196.

14 Pruss H, Finke C, Holtje M, Hofmann J, Klingbeil C, Probst C et al. N-methyl-Daspartate receptor antibodies in herpes simplex encephalitis. Ann Neurol 2012; 72 902-911.

15 Steiner J, Walter M, Glanz W, Sarnyai Z, Bernstein HG, Vielhaber S et al. Increased prevalence of diverse $\mathrm{N}$-methyl-D-aspartate glutamate receptor antibodies in patients with an initial diagnosis of schizophrenia: specific relevance of IgG NR1a antibodies for distinction from N-methyl-D-aspartate glutamate receptor encephalitis. JAMA Psychiatry 2013; 70: 271-278.

16 Begemann M, Grube S, Papiol S, Malzahn D, Krampe H, Ribbe K et al. Modification of cognitive performance in schizophrenia by complexin 2 gene polymorphisms. Arch Gen Psychiatry 2010; 67: 879-888.

17 Ribbe K, Friedrichs H, Begemann M, Grube S, Papiol S, Kastner A et al. The crosssectional GRAS sample: a comprehensive phenotypical data collection of schizophrenic patients. BMC Psychiatry 2010; 10: 91.

18 Wandinger KP, Saschenbrecker S, Stoecker W, Dalmau J. Anti-NMDA-receptor encephalitis: a severe, multistage, treatable disorder presenting with psychosis. J Neuroimmunol 2011; 231: 86-91.

19 Toyka KV, Brachman DB, Pestronk A, Kao I. Myasthenia gravis: passive transfer from man to mouse. Science 1975; 190: 397-399.

20 Piedrahita JA, Zhang SH, Hagaman JR, Oliver PM, Maeda N. Generation of mice carrying a mutant apolipoprotein $\mathrm{E}$ gene inactivated by gene targeting in embryonic stem cells. Proc Natl Acad Sci USA 1992; 89: 4471-4475.

21 Radyushkin K, El-Kordi A, Boretius S, Castaneda S, Ronnenberg A, Reim K et al. Complexin2 null mutation requires a 'second hit' for induction of phenotypic changes relevant to schizophrenia. Genes Brain Behav 2010; 9: 592-602.

22 Purcell S, Neale B, Todd-Brown K, Thomas L, Ferreira MA, Bender D et al. PLINK: a tool set for whole-genome association and population-based linkage analyses. Am J Hum Genet 2007; 81: 559-575.

23 Fullerton SM, Shirman GA, Strittmatter WJ, Matthew WD. Impairment of the blood-nerve and blood-brain barriers in apolipoprotein e knockout mice. Exp Neurol 2001; 169: 13-22.
Neuropsychiatric disease relevance of circulating NMDAR-AB

C Hammer et al

24 Saher G, Rudolphi F, Corthals K, Ruhwedel T, Schmidt KF, Lowel S et al. Therapy of Pelizaeus-Merzbacher disease in mice by feeding a cholesterol-enriched diet. Nat Med 2012; 18: 1130-1135.

25 Bell RD, Winkler EA, Singh I, Sagare AP, Deane R, Wu Z et al. Apolipoprotein E controls cerebrovascular integrity via cyclophilin A. Nature 2012; 485: $512-516$.

26 Shlosberg D, Benifla M, Kaufer D, Friedman A. Blood-brain barrier breakdown as a therapeutic target in traumatic brain injury. Nat Rev Neurol 2010; 6 393-403.

27 Baburamani AA, Ek CJ, Walker DW, Castillo-Melendez M. Vulnerability of the developing brain to hypoxic-ischemic damage: contribution of the cerebral vasculature to injury and repair? Front Physiol 2012; 3: 424.

28 Visscher PM, Brown MA, McCarthy MI, Yang J. Five years of GWAS discovery. Am J Hum Genet 2012; 90: 7-24.

29 Zheng S, Eacker SM, Hong SJ, Gronostajski RM, Dawson TM, Dawson VL. NMDAinduced neuronal survival is mediated through nuclear factor $1-A$ in mice. $J$ Clin Invest 2010; 120: 2446-2456.

30 Sfriso P, Ghirardello A, Botsios C, Tonon M, Zen M, Bassi $\mathrm{N}$ et al. Infections and autoimmunity: the multifaceted relationship. J Leukoc Biol 2010; 87 385-395.

31 Ascherio A, Munger KL, Lunemann JD. The initiation and prevention of multiple sclerosis. Nat Rev Neurol 2012; 8: 602-612.

32 Dale RC, Irani SR, Brilot F, Pillai S, Webster R, Gill D et al. N-methyl-D-aspartate receptor antibodies in pediatric dyskinetic encephalitis lethargica. Ann Neurol 2009; 66: 704-709.

33 Tan A, Shuey N, Bladin C. A modern perspective on the differential diagnosis between encephalitis lethargica or anti-NMDA-receptor encephalitis. J Clin Neurosci 2010; 17: 1204-1206.

34 Reiber H, Peter JB. Cerebrospinal fluid analysis: disease-related data patterns and evaluation programs. J Neurol Sci 2001; 184: 101-122.

35 Ek CJ, Dziegielewska KM, Habgood MD, Saunders NR. Barriers in the developing brain and Neurotoxicology. Neurotoxicology 2012; 33: 586-604.

36 Grzeda E, Wisniewska RJ, Wisniewski K. Effect of an NMDA receptor agonist on T-maze and passive avoidance test in 12-week streptozotocin-induced diabetic rats. Pharmacol Rep 2007; 59: 656-663.

37 Sharp FR, Hendren RL. Psychosis: atypical limbic epilepsy versus limbic hyperexcitability with onset at puberty? Epilepsy Behav 2007; 10: 515-520.

38 Olney JW, Farber NB. Glutamate receptor dysfunction and schizophrenia. Arch Gen Psychiatry 1995; 52: 998-1007.

39 Manto M, Dalmau J, Didelot A, Rogemond V, Honnorat J. Afferent facilitation of corticomotor responses is increased by lgGs of patients with NMDA-receptor antibodies. J Neurol 2011; 258: 27-33.

40 Bechter K, Reiber H, Herzog S, Fuchs D, Tumani H, Maxeiner HG. Cerebrospinal fluid analysis in affective and schizophrenic spectrum disorders: identification of subgroups with immune responses and blood-CSF barrier dysfunction. J Psychiat Res 2010; 44: 321-330.

41 Doria A, Canova M, Tonon M, Zen M, Rampudda E, Bassi N et al. Infections as triggers and complications of systemic lupus erythematosus. Autoimmun Rev 2008; 8: 24-28.

42 Randone SB, Guiducci S, Cerinic MM. Systemic sclerosis and infections. Auto immun Rev 2008; 8: 36-40.

43 Blanpied TA, Clarke RJ, Johnson JW. Amantadine inhibits NMDA receptors by accelerating channel closure during channel block. I Neurosci 2005; 25: 3312-3322

44 Larrauri A, de Mateo S. Characterisation of swabbing for virological analysis in the Spanish Influenza Sentinel Surveillance System during four influenza seasons in the period 2002-2006. Euro Surveill 2007; 12: E5-E6.

45 Avitsur R, Mays JW, Sheridan JF. Sex differences in the response to influenza virus infection: modulation by stress. Horm Behav 2011; 59: 257-264.

Supplementary Information accompanies the paper on the Molecular Psychiatry website (http://www.nature.com/mp) 


\title{
Genetic Markers of a Munc13 Protein Family Member, BAIAP3, Are Gender Specifically Associated with Anxiety and Benzodiazepine Abuse in Mice and Humans
}

\author{
Sonja M Wojcik, ${ }^{1 *}$ Martesa Tantra, ${ }^{2,3^{*}}$ Beata Stepniak, ${ }^{2 *}$ Kwun-nok M Mann, ${ }^{1,3}$ Katja Müller-Ribbe, ${ }^{2}$ \\ Martin Begemann, ${ }^{2}$ Anes Ju, ${ }^{2}$ Sergi Papiol,, 3 Anja Ronnenberg, ${ }^{2}$ Artem Gurvich, ${ }^{2}$ Yong Shin, ${ }^{1,4}$ \\ Iris Augustin, ${ }^{1,5}$ Nils Brose, ${ }^{1,3}$ and Hannelore Ehrenreich ${ }^{2,3}$
}

${ }^{1}$ Max Planck Institute of Experimental Medicine, Department of Molecular Neurobiology, Göttingen, Germany; ${ }^{2}$ Max Planck Institute of Experimental Medicine, Clinical Neuroscience, Göttingen, Germany; ${ }^{3}$ DFG Center for Nanoscale Microscopy and Molecular Physiology of the Brain, Göttingen, Germany; ${ }^{4}$ present address: BioElectronics, Institute of Microelectronics, Agency for Science, Technology and Research (A*STAR), Singapore, Singapore; and ${ }^{5}$ present address: German Cancer Research Center, Department Signaling and Functional Genomics, Heidelberg, Germany

\begin{abstract}
Anxiety disorders and substance abuse, including benzodiazepine use disorder, frequently occur together. Unfortunately, treatment of anxiety disorders still includes benzodiazepines, and patients with an existing comorbid benzodiazepine use disorder or a genetic susceptibility for benzodiazepine use disorder may be at risk of adverse treatment outcomes. The identification of genetic predictors for anxiety disorders, and especially for benzodiazepine use disorder, could aid the selection of the best treatment option and improve clinical outcomes. The brain-specific angiogenesis inhibitor L-associated protein 3 (Baiap3) is a member of the mammalian uncoordinated 13 (Munc13) protein family of synaptic regulators of neurotransmitter exocytosis, with a striking expression pattern in amygdalae, hypothalamus and periaqueductal gray. Deletion of Baiap3 in mice leads to enhanced seizure propensity and increased anxiety, with the latter being more pronounced in female than in male animals. We hypothesized that genetic variation in human BAIAP3 may also be associated with anxiety. By using a phenotype-based genetic association study, we identified two human BAIAP3 single-nucleotide polymorphism risk genotypes (AA for rs2235632, $\pi$ for rs 1132358 ) that show a significant association with anxiety in women and, surprisingly, with benzodiazepine abuse in men. Returning to mice, we found that male, but not female, Baiap3 knockout (KO) mice develop tolerance to diazepam more quickly than control animals. Analysis of cultured Baiap3 KO hypothalamus slices revealed an increase in basal network activity and an altered response to diazepam withdrawal. Thus, Baiap3/BAIAP3 is gender specifically associated with anxiety and benzodiazepine use disorder, and the analysis of Baiap3/BAIAP3-related functions may help elucidate mechanisms underlying the development of both disorders.

Online address: http://www.molmed.org

doi: $10.2119 /$ molmed.2013.00033
\end{abstract}

\section{INTRODUCTION}

Anxiety disorders have high lifetime prevalence rates (1) and exhibit a remarkable comorbidity with substance use disorders (2-4). This association worsens treatment outcomes for both conditions (5) and represents a significant burden on individuals and society. Both anxiety disorders and substance use disorders are complex disorders that arise from a combination of genetic influence and environmental factors. To

*SMW, MT, and BS contributed equally to this study.

Address correspondence to Sonja M Wojcik, Max Planck Institute of Experimental Medicine, Department of Molecular Neurobiology, Hermann-Rein-Str. 3, D-37075 Göttingen, Germany.Phone: +49-551-3899-722; Fax: +49-551-3899-715; E-mail: wojcik@em.mpg.de. Submitted April 12, 2013; Accepted for publication May 14, 2013; Epub (www.molmed.org) ahead of print May 14, 2013.

The Feinstein Institute North
for Medical Research Shore LI)

improve upon established treatment options, which include pharmacological as well as cognitive-behavioral therapies $(6,7)$, a more detailed picture of the etiology of these disorders is instrumental. Estimates of heritability from twin and family studies are in the range of $20-40 \%$ across the different anxiety disorders $(8,9)$ and in the range of $40-70 \%$ for the major substance use disorders (10). Recent studies point to the involvement of a large number of genes with relatively small effect sizes for both anxiety disorder $(11,12)$ and substance use disorder (13-15). Although the interaction between anxiety disorders and substance use disorders is likely bidirec- 
tional and varies by the type of anxiety (16), genetically determined anxiousness personality traits may make the development of an addiction more likely $(2,17-19)$. The recommended first-line pharmacological treatments of anxiety disorders are selective serotonin or serotonin/norepinephrine reuptake inhibitors and the calcium channel modulator pregabaline (6). However, primary care physicians often still prescribe benzodiazepines, which rank among the most frequently abused prescription medications (National Institute on Drug Abuse [http://www.nida.nih.gov]), to patients suffering from anxiety disorders (20). Identifying genetic risk markers would advance our understanding of the biology of anxiety and benzodiazepine abuse and would be a valuable step in improving treatment options for these complex diseases.

In addition to human family, twin and genome-wide association studies, animal models are used to study the genetic basis and neural circuitries of anxiety and addiction. For both animals and humans, anxiety is an adaptive defensive response to threatening stimuli necessary for the survival of the species, whereas anxiety disorders are an extreme and maladaptive manifestation of normal anxiety (21). Somatic anxiety symptoms are mediated by the release of specific neurotransmitters and neuropeptides. The selection of candidate genes that are being investigated in animal studies is still largely driven by hypotheses of the neural circuitries and neurotransmitter systems thought to be involved in mediating fear and anxiety (22). By using a candidate gene approach, we investigate the involvement of the brain-specific angiogenesis inhibitor I-associated protein 3 (Baiap3), which is highly expressed in brain regions involved in processing fear, such as the amygdalae, hypothalamus and periaqueductal gray, in behavioral phenotypes relevant for human psychiatric disorders.

Baiap3 is a member of the mammalian uncoordinated 13 (Munc13) family of synaptic regulators of neurotransmitter exocytosis (23-25). Baiap3 has a unique and striking expression pattern (Allen Brain Atlas [http://mouse.brainmap.org]) in brain regions such as the central, medial and basomedial amygdaloid nuclei; the hypothalamus; and the periaqueductal gray. These areas are involved in regulating autonomic functions and are also critical in processing fearful stimuli and mediating anxiety-related behaviors $(26,27)$. The cellular function of Baiap3 is currently unknown; however, all other Munc13 members are regulators of vesicle exocytosis in various cell types (28). In the brain, Munc13-1 and

Munc13-2 are essential for membrane fusion of synaptic vesicles containing classical neurotransmitters, such as glutamate or $\gamma$-aminobutyric acid (GABA) (25). Munc13-4, a non-neuronal Munc13 isoform most closely related to Baiap3 at the sequence level, is involved in exocytosis in cells of the hematopoietic system $(29,30)$.

To explore the function of Baiap3, we combined the behavioral analysis of Baiap3 knockout (KO) mice with a phenotype-based genetic association study (PGAS) of the human BAIAP3 gene by using the Göttingen Research Association for Schizophrenia (GRAS) database $(31,32)$. Using this two-pronged approach, we identify Baiap3/BAIAP3 as the first genetic risk marker for anxiety and benzodiazepine abuse in both mice and humans.

\section{MATERIALS AND METHODS}

\section{Animals}

Animal maintenance. All experiments were approved by the local Animal Care and Use Committee of Lower Saxony, Oldenburg, Germany. The first three coding exons of the murine Baiap3 gene were preplaced with a neomycin resistance cassette through homologous recombination in embryonic stem cells (129/Ola) (Supplementary Figure S1A). Baiap3 mutant mice of mixed 129/Ola;C57BL/6N background were backcrossed for seven more generations to C57BL/ $6 \mathrm{~N}$; all experiments were done with WT and KO littermates of the resulting generation 8 . After weaning, mice were group-housed in standard plastic cages $(n=5$ per cage) and maintained in a temperaturecontrolled environment $\left(21 \pm 2^{\circ} \mathrm{C}\right)$ on a 12-h light-dark cycle with food and water ad libitum, unless stated otherwise.

Drugs used in animal experiments. Two classical benzodiazepines, positive allosteric modulators of GABA type A receptors $\left(\mathrm{GABA}_{\mathrm{A}} \mathrm{R}\right)$ were used: (i) the long-acting benzodiazepine diazepam (ratiopharm GmbH, Ulm, Germany) was suspended in saline containing polysorbate 80 for intraperitoneal (IP) injection, and (ii) the short-acting benzodiazepine midazolam (ratiopharm) was added to $2 \%$ sucrose solution for oral administration. Antagonists used were as follows: (i) flumazenil (Sigma-Aldrich Chemie, Munich, Germany), routinely applied in the clinic to counteract benzodiazepine overdoses, was dissolved in saline containing polysorbate 80 and $\mathrm{HCl}$; and (ii) pentylenetetrazole (PTZ) (Sigma-Aldrich Chemie), a noncompetitive GABA antagonist with epileptogenic properties, was dissolved in saline for IP injection.

Phenotypical characterization of Baiap3 KO mice. Behavioral characterization of naive Baiap3 $\mathrm{KO}$ mice and their WT littermates of both sexes began at the age of $8 \mathrm{wks}$ and was performed in the following order: elevated plus-maze, open field, light-dark box, hole board, rotarod and exposure to a fear-conditioning chamber to assess novelty-induced freezing behavior. Mouse numbers of all individual experiments are given in the figure legends.

Elevated plus-maze. The mouse was placed in the central platform, facing an open arm of the plus-maze. Behavior was recorded over $5 \mathrm{~min}$ by an overhead video camera. A personal computer equipped with Viewer software (Biobserve, Bonn, Germany) was used to calculate the time each animal spent in open versus closed arms. The proportion of time spent in open arms (natural aversion) was used as a fear equivalent.

Open field. Spontaneous activity in open field was tested in a gray Perspex 
arena (120 cm in diameter, $25 \mathrm{~cm}$ high), virtually divided into three zones: central, intermediate and peripheral. The mouse was placed in the center, and the test was started when the mouse reached the wall. Over $7 \mathrm{~min}$, the mouse was allowed to freely explore the open field. Behavior was recorded by a personal computer-linked overhead video camera and calculated using Viewer software. Readouts were as follows: velocity, distance traveled, time spent in each zone and initial latency to reach the wall.

Hole board. The hole board apparatus (TSE Systems GmbH, Bad Homburg, Germany) for measuring exploratory activity consisted of a $50 \mathrm{~cm} \times 50 \mathrm{~cm} \times$ $35 \mathrm{~cm}$ transparent Perspex chamber with a nontransparent floor raised above the bottom of the chamber. The floor had 16 equally spaced holes, $2.4 \mathrm{~cm}$ in diameter, fitted with a light barrier sensor $(8 \mathrm{~mm}$ below floor). Mice were allowed to explore the chamber for $5 \mathrm{~min}$, and the number of explored holes (head dips) was recorded.

Rotarod. This test for motor function, balance and coordination consists of a rotating drum (Ugo Basile, Comerio, Varese, Italy), accelerated from 4 to 40 revolutions per minute over $5 \mathrm{~min}$. Each mouse was placed individually on a drum and the latency of falling from the drum was recorded using a stopwatch.

To assess motor learning, the test was repeated $24 \mathrm{~h}$ later.

Novelty-induced fear response. To assess novelty-induced fear response (indicated by freezing behavior), a chamber designed for training and testing of context fear conditioning was used. Mice were placed inside the chamber and allowed to explore the chamber freely for 2 min, during which time no additional stimulus was presented (equivalent to the assessment of baseline freezing of the fear-conditioning paradigm). Duration of freezing behavior, defined as the absolute lack of movement (excluding respiratory movements), was recorded by a video camera and a personal computer equipped with Video Freeze software (MED Associates, St. Albans, VT, USA).
Pentylenetetrazole-induced seizures. Seizure activity was induced in wakeful mice by using a single IP injection of PTZ (50 mg/ $\mathrm{kg}$ body weight) (33). After injection of the compound, the mouse was placed in a small, clear home cage and closely observed for $30 \mathrm{~min}$. Latencies to focal (partial clonic), generalized (generalized clonic) and maximal (tonic-clonic) behavioral seizures were recorded. Furthermore, four phases in the continuum of behavioral response to IP PTZ injection were defined as follows: (i) hypoactivity (progressive decrease in motor activity until the animal came to rest in a crouched or prone position with the abdomen in full contact with the cage bottom); (ii) partial clonus (clonus seizure activity affecting face, head and/or forelimb or forelimbs); (iii) generalized clonus (sudden loss of upright posture, whole body clonus involving all four limbs and tail, rearing and autonomic signs); and (iv) tonic-clonic (maximal) seizure (generalized seizure characterized by tonic hindlimb extension-also associated with death). Finally, latencies to partial clonus (PC), generalized clonus (GC) and tonic-clonic (TC) seizures were summed to assign a seizure score to each mouse, used as a quantitative trait measure for mapping according to the following equation: seizure score $=[(0.2) \times$ $(1 /$ PC latency $)+(0.3) \times(1 /$ GC latency $)+$ $(0.5) \times(1 /$ TC latency $)] \times 1,000$. The weighting factors $(0.2,0.3$ and 0.5$)$ in the equation were included as a means of incorporating a measure of the progressive nature of the PTZ-induced seizure phenotype into the severity rating because generalized clonus is regarded as a more significant event than partial clonus and tonic hind limb extension as the most severe component of the phenotype. Therefore, the seizure score reflects the degree of progression of the seizure phenotype in each mouse (33).

Diazepam dependence, tolerance and withdrawal. The mice received injections of diazepam ( $5 \mathrm{mg} / \mathrm{kg}$ body weight IP) for 10 consecutive days. Rotarod test was performed $30 \mathrm{~min}$ after each diazepam injection for $7 \mathrm{~d}$, with baseline rotarod training performed for $2 \mathrm{~d}$ before starting injections. On d 11, diazepam withdrawal was induced by flumazenil (15 mg/kg body weight IP), followed by injection of PTZ ( $50 \mathrm{mg} / \mathrm{kg}$ body weight IP) to induce withdrawal-related seizures. Seizure induction by PTZ (50 mg/ kg body weight IP) was also performed on drug-naive mice.

Midazolam oral self-administration and behavior testing in the addicted state. To induce benzodiazepine dependence as a prerequisite for oral selfadministration (document of addiction), group-housed mice received midazolam (ratiopharm) in 2\% sucrose (to reduce the bitter taste), instead of drinking water. Midazolam concentration was increased weekly, starting from $0.005 \mathrm{mg} / \mathrm{mL}$ until the maximum concentration of $0.05 \mathrm{mg} / \mathrm{mL}$ was reached after $10 \mathrm{wks}$. A respective control group received $2 \%$ sucrose only. One set of midazolam mice was then exposed to a midazolam preference test. For this purpose, mice were first switched to single housing with a continued supply of midazolam $(0.05 \mathrm{mg} / \mathrm{mL})$ for $2 \mathrm{wks}$. For the preference test, every mouse had a choice of two bottles containing either midazolam $(0.05 \mathrm{mg} / \mathrm{mL})$ in $2 \%$ sucrose or $2 \%$ sucrose alone for another $2 \mathrm{wks}$. The relative consumption of midazolam solution was calculated. The other set of mice (midazolam and control mice) stayed group-housed and underwent automated home cage observation using the LABORAS $^{\mathrm{TM}}$ system (Metris, Hoofddorp, Netherlands). LABORAS is a fully automated system for continuous behavior recognition and tracking in small rodents. For habituation before testing, mice were temporarily put in single cages similar to the LABORAS cage in the testing room for 2 consecutive nights (1700 to 0900). On the day of testing, Makrolon type 3 cages $\left(840 \mathrm{~cm}^{2}\right)$, filled with a 2 -cm layer of bedding used during the habituation phase, were placed on each triangular sensor platform $(95 \mathrm{~cm} \times 75 \mathrm{~cm} \times 75 \mathrm{~cm})$. Food and sucrose solution with midazolam (addicted group) or $2 \%$ sucrose (control group) were provided ad libitum. 
Before each session, LABORAS was calibrated by using the calibration procedure and reference weights supplied by Metris. Movements during nighttime (1800 to 0900) were recorded and distinguished as separate behavioral patterns by the LABORAS software. Locomotion duration and scratching frequency during the dark phase (2000 to 0800) was analyzed.

Statistical analysis. Behavioral data were analyzed separately for males and females by the Mann-Whitney $U$ test and two-way analysis of variance (ANOVA), including post hoc Bonferroni testing, where applicable, using Prism 4 (GraphPad Software, San Diego, CA, USA). Significance level was set to $p<0.05$. All data are presented as mean \pm standard error of the mean (SEM).

\section{Human Sample}

Schizophrenic patient sample. The schizophrenic patient sample $(\mathrm{n}=1,086)$ was recruited across 23 sites throughout Germany in the cross-sectional GRAS study and most comprehensively phenotyped $(31,32)$. The study was approved by the Ethics Committee of the GeorgAugust-University (Göttingen, Germany) and the review boards of participating centers and complies with the Declaration of Helsinki. Patients fulfilling Diagnostic and Statistical Manual of Mental Disorders: DSM-IV-TR, 4th edition, text revision (34) criteria for schizophrenia or schizoaffective disorder were included in the analyses regardless of their disease stage (acute, chronic, residual or remitted). Almost all patients were of European Caucasian descent (Caucasian $94.7 \%$; other ethnicities $1.9 \%$; unknown $3.4 \%)$.

Healthy control sample. Voluntary blood donors $(n=1,142)$ recruited following the national guidelines for blood donation were included for case control analysis $(31,32)$. Also the majority of control subjects are of European Caucasian ethnicity (Caucasian $97.8 \%$; other ethnicities $2 \%$; unknown $0.2 \%$ ).

Sociodemographic and clinical variables. Sociodemographic data (age, years of education, level of unemployment), information on substance use disorder (summarizing abuse and dependence based on the DSM-IV-TR criteria for alcohol and cannabis) and clinical variables describing disease severity were used to characterize the sample. Clinical variables included Positive and Negative Syndrome Scale (PANSS) positive scale as a measure of positive symptom severity (35) as well as chlorpromazine equivalents to estimate the relative dose of antipsychotic medication. The Global Assessment of Functioning (GAF) scale (DSM-IV-TR) was used as a measure of impairment of psychological, social and occupational functioning.

Target variables. The dichotomous DSM-IV-TR benzodiazepine use disorder diagnosis (summarizing abuse and dependence) and the quantitative anxiety composite score were our target variables. The anxiety composite score is based on the aggregation of four anxietyrelated variables: (i) Brief Symptom Inventory (BSI) subscale anxiousness; (ii) State-Trait Anxiety Inventory (STAI) subscale trait anxiety; (iii) STAI subscale state anxiety; and (iv) anxiety item of the PANSS general psychopathology subscale (Supplementary Figure S2).

DNA extraction and normalization. Genomic DNA was purified from whole blood by using JETQUICK Blood and Cell Culture DNA Spin Kit (Genomed, Loehne, Germany) according to the manufacturer's protocol. DNA aliquots were stored at $-80^{\circ} \mathrm{C}$. For further analyses, DNA was normalized to $50 \mathrm{ng} / \mu \mathrm{L}$ with an automated robotic platform (Microlab Star, Hamilton, Bonaduz, Switzerland). Each sample was analyzed with a $0.8 \%$ agarose gel for quality control.

Genotyping. The three selected SNPs (rs11648169, rs2235632, rs1132358) of BAIAP3 were analyzed by using Simple Probes (TIB Molbiol, Berlin, Germany) and genotyped using the LightCycler 480 Genotyping Software implemented in the LightCycler 480 system (Roche, Mannheim, Germany). The reaction mixture $(10 \mu \mathrm{L})$ was prepared with $20 \mathrm{ng}$ DNA in 384-well plates following the standard protocol (Roche). In each run, eight positive controls (hgDNA, Bioline, Luckenwalde, Germany) and negative water blanks were included for quality and internal control purposes. Of the GRAS patients, a total of $n=1,082$ $(99.63 \%)$ were successfully genotyped for BAIAP3 SNP1 (C/G) rs11648169, $\mathrm{n}=$ $1,086(100 \%)$ for BAIAP3 SNP2 (G/A) rs2235632 and $n=1,069(98.43 \%)$ for BAIAP3 SNP3 (C/T) rs1132358 and included in the analyses. Of the healthy control subjects, all $\mathrm{n}=1,142$ were successfully genotyped for SNP1, SNP2 and SNP3 of the BAIAP3 gene.

Statistical analyses. For all analyses, statistical significance was set to 0.05 . Statistical analyses of human data were performed by using SPSS for Windows, version 17.0. Group differences in categorical and continuous variables were assessed using $\chi^{2}$ or Mann-Whitney $U$ tests; in cases of normal distribution of the continuous variable, $t$ tests were performed. Anxiety score composition was done using $z$-standardized mean subscale scores (BSI anxiousness, STAI trait anxiety, STAI state anxiety) or, in the case of PANSS anxiety, a $z$-standardized single item, organized such that higher values represent higher symptom severity. Intercorrelations and internal consistency of the anxiety composite score was calculated by using Pearson correlation coefficient and Cronbach $\alpha$ (36). In the GRAS sample, the following items or scales were incomplete: BSI anxiousness $7.5 \%$ missing, STAI trait anxiety $20.2 \%$, STAI state anxiety $21.6 \%$ and PANSS anxiety $3.2 \%$. If all four anxiety variables were available, the mean was calculated for each respective subject as an individual anxiety composite score. In the case of missing data, a linear regression-based multiple imputation model (10 iterations) of missing data was applied, if at least three out of the four variables per subject were available. For the 190 individuals with imputed values, the final anxiety composite score represents the mean of 10 imputed values for the missing item, increasing the availability of the anxiety score from $n=771$ to $n=961$ schizo- 
phrenic subjects (37). Analysis of covariance (adjusted for age, PANSS positive subscale score and chlorpromazine equivalents) was used to analyze the effect of SNP genotypes on the standardized anxiety composite score. For the phenotype-genotype association analyses (including peripheral blood mononuclear cells [PBMCs]; see below) of the BAIAP3 SNP rs2235632, G carriers (GG and AG) were aggregated and contrasted with individuals homozygous for the A allele, and in the case of SNP rs1132358, C carriers (CC and TC) were aggregated and contrasted with TT individuals. SNP rs11648169 was excluded from further analyses, since it yielded no statistically significant effects.

\section{In Vitro Analyses}

Immunofluorescence analysis. Brains were perfusion-fixed, and organotypic hypothalamus slices were immersion fixed in $4 \%$ paraformaldehyde in phosphate buffer ( $\mathrm{pH}$ 7.4). Brains were postfixed for $1 \mathrm{~h}$, cryoprotected with $30 \%$ sucrose and frozen. For immunofluorescence analysis, free-floating brain sections of $40 \mu \mathrm{m}$ thickness or organotypic sections of $300 \mu \mathrm{m}$ thickness were incubated in primary antibodies for $72 \mathrm{~h}$ followed by incubation with IgG-coupled Alexa Fluor 488, Alexa Fluor 555 and Alexa Flour 633 dyes (Invitrogen [Life Technologies, Darmstadt, Germany]) for $24 \mathrm{~h}$. Rabbit and guinea pig antibodies to Baiap3 were raised to a purified fragment (amino acids 617-973) containing the munc homology domain (MHD)-1 and MHD-2 of mouse Baiap3. Commercial primary antibodies used were rabbit and guinea pig anti-vesicular glutamate transporter 1 (VGLUT1), rabbit and guinea pig anti-VGLUT2, rabbit and guinea pig anti-vesicular inhibitory amino acid transporter (Viaat), mouse anti-Gephyrin (mAB7a) (all from Synaptic Systems, Göttingen, Germany), and mouse anti-postsynaptic density protein 95 (PSD-95) (clone K28/48, NeuroMab). False color images of brain sections and organotypic slices were obtained with a fluorescence stereomicroscope (Leica
FluoCombi III ${ }^{\mathrm{TM}}$ ) and an ApoTome ${ }^{\mathrm{TM}}$ fluorescence microscope (Axio Imager Z1; Zeiss), respectively.

Hypothalamus slice culture. Organotypic hypothalamus slices of $300-\mu \mathrm{m}$ thickness from postnatal d 5 (P5) and P6 mice were prepared in Hanks balanced salt solution (24020-091; Invitrogen [Life Technologies]) with $20 \%$ glucose and $1 \mathrm{mmol} / \mathrm{L}$ kynurenic acid (SigmaAldrich, Germany) (pH 7.4), by using a McIlwain Tissue Chopper. Slices were cultured in six-well plates on confetti cut from 0.45- $\mu \mathrm{m}$ filters (FHLC04700; EMD Millipore [Millipore Ireland B.V., Tullagreen, Carrigtwohill County Cork, Ireland]) that were placed in $0.4-\mu \mathrm{m}$ Millicell cell culture inserts (PICM03050; Millipore) for $5 \mathrm{~d}$ using a mixture of $41 \%$ Earle basal medium Eagle (BME) (F 0225; Biochrom, Berlin, Germany), with 25\% Earle balanced salt solution $(1.8 \mathrm{mmol} / \mathrm{L}$ $\mathrm{CaCl}_{2}, 1 \mathrm{mmol} / \mathrm{L} \mathrm{NaH} \mathrm{PO}_{4}, 0.8 \mathrm{mmol} / \mathrm{L}$ $\mathrm{MgSO}_{4}, 116 \mathrm{mmol} / \mathrm{L} \mathrm{NaCl}, 26.2 \mathrm{mmol} / \mathrm{L}$ $\mathrm{NaHCO}_{3}, 5.4 \mathrm{mmol} / \mathrm{L} \mathrm{KCl}, 5 \mathrm{mmol} / \mathrm{L} \mathrm{glu}-$ cose), $20 \%$ heat-inactivated horse serum, $10 \% \mathrm{H}_{2} \mathrm{O}, 25 \mathrm{mmol} / \mathrm{L}$ 4-(2-hydroxyethyl)1-piperazineethanesulfonic acid (HEPES) (Biochrom), $28 \mathrm{mmol} / \mathrm{L}$ glucose, $1 \mathrm{mmol} / \mathrm{L}$ GlutaMAX ${ }^{\mathrm{TM}}$ (35050; Invitrogen [Life Technologies]), $1 \mu \mathrm{g} / \mathrm{mL}$ insulin, $88 \mu \mathrm{g} / \mathrm{mL}$ ascorbic acid, $0.25 \%$ MEM Vitamine Solution (K0373; Biochrom) and 0.5\% MEM Amino Acids (K0363; Biochrom). On d 5 in vitro, the cultures were switched to a medium with identical components but containing $5 \%$ horse serum, 55\% BME and $2 \mathrm{mmol} / \mathrm{L}$ GlutaMAX. At the same time, diazepam was added to the medium from a $60 \mathrm{mmol} / \mathrm{L}$ stock solution in dimethylsulfoxide (DMSO) for a final concentration of $10 \mu \mathrm{mol} / \mathrm{L}$. For control cultures, DMSO was added as a vehicle control at the same dilution of 1:6,000. The $\mathrm{CO}_{2}$ concentration was $5 \%$, and medium changes were done on the day after culture and every $48 \mathrm{~h}$ after that.

Electrophysiological analyses. Organotypic slices containing the ventromedial hypothalamus were transferred to the recording chamber between DIV10 and DIV17. Recordings were started after a 30-min recovery time, the extracellular recording solution contained $120 \mathrm{mmol} / \mathrm{L}$ $\mathrm{NaCl}, 26 \mathrm{mmol} / \mathrm{L} \mathrm{NaHCO}_{3}, 1 \mathrm{mmol} / \mathrm{L}$ $\mathrm{KH}_{2} \mathrm{PO}_{4}, 2 \mathrm{mmol} / \mathrm{L} \mathrm{KCl}, 20 \mathrm{mmol} / \mathrm{L} \mathrm{glu}-$ cose, $2 \mathrm{mmol} / \mathrm{L} \mathrm{MgCl}_{2}, 2 \mathrm{mmol} / \mathrm{L} \mathrm{CaCl}_{2}$ and $250 \mathrm{nmol} / \mathrm{L}$ flumazenil. Cells were whole-cell voltage clamped at -70 or $-20 \mathrm{mV}$ or recorded in current clamp mode with an EPC 10 USB Double (HEKA, Lambrecht/Pfalz, Germany) under control of the Patchmaster 2.52 program (HEKA). All analyses were performed by using the Mini Analysis Program (Synaptosoft, Decatur, GA, USA). Recordings of miniature inhibitory postsynaptic currents (mIPSCs) were performed in the presence of $1 \mu \mathrm{mol} / \mathrm{L}$ tetrodotoxin (Tocris [R\&D Systems, Wiesbaden-Nordenstadt, Germany]) and $10 \mu \mathrm{mol} / \mathrm{L} 2,3$-dioxo-6-nitro-1,2,3,4tetrahydrobenzo[ $f$ quinoxaline-7sulfonamide (NBQX) (Tocris [R\&D Systems]), with an intracellular solution containing $100 \mathrm{mmol} / \mathrm{L} \mathrm{KCl}, 50 \mathrm{mmol} / \mathrm{L}$ K-gluconate, $10 \mathrm{mmol} / \mathrm{L}$ HEPES, $0.1 \mathrm{mmol} / \mathrm{L}$ EGTA, $0.3 \mathrm{mmol} / \mathrm{L}$ GTP, $4 \mathrm{mmol} / \mathrm{L}$ ATP and $0.2 \%$ biocytin. Action potentials and spontaneous inhibitory postsynaptic currents (IPSCs) were recorded with an intracellular solution containing $20 \mathrm{mmol} / \mathrm{L} \mathrm{KCl}, 130 \mathrm{mmol} / \mathrm{L} \mathrm{K}$-gluconate, $10 \mathrm{mmol} / \mathrm{L}$ HEPES, $0.1 \mathrm{mmol} / \mathrm{L}$ EGTA, $0.3 \mathrm{mmol} / \mathrm{L} \mathrm{GTP}, 4 \mathrm{mmol} / \mathrm{L}$ ATP and $0.2 \%$ biocytin. Action potentials analyzed were from the first minute of a 2-min recording; membrane potentials were measured after setting the current injection to $0 \mathrm{pA}$ at the end of the recording. IPSCs were recorded for $5 \mathrm{~min}$ after switching the cell to a holding potential of $-20 \mathrm{mV}$ and waiting for $1 \mathrm{~min}$. Statistical analyses were performed using GraphPad Prism5.

Analysis of BAIAP3 mRNA levels in PBMCs. PBMCs from 121 patients were isolated by using the standard FicollPaque Plus isolation procedure (GE Healthcare, Munich, Germany). For RNA isolation, the miRNeasy Mini Kit (Qiagen, Hilden, Germany) was used. A total of $1 \mu \mathrm{g}$ RNA, a mixture of oligo dT, hexamer primers, dNTPS (10 mmol/L each) and SuperScriptIII (200 U; Invitrogen [Life Technologies]) were used for transcription into cDNA $(20-\mu \mathrm{L}$ reaction). The mixture 
was incubated for $10 \mathrm{~min}$ at $25^{\circ} \mathrm{C}$ and 45 min at $50^{\circ} \mathrm{C}$, followed by $45 \mathrm{~min}$ at $55^{\circ} \mathrm{C}$. For the quantitative reverse transcriptase polymerase chain reaction (qRT-PCR), a 1:10 dilution of the cDNA was used and three replicate experiments per sample were performed: $5 \mu \mathrm{L}$ Power SYBR mix (Applied Biosystems) and $1 \mathrm{pmol}$ of each primer were added. BAIAP3 qRTPCR primers used were as follows: $5^{\prime}$ AGCTGGGCCCACCGCATCTCT-3' with 5'-CTCGGCAGGCACGGAAAAGTAG-3' and 5'-CTGACTTCAACAGCGACACC-3' with 5'-TGCTGTAGCCAAATTCGTTGT-3'. The following cycling profile was run on the LightCycler480 system (Roche): preheating at $95^{\circ} \mathrm{C}$ for $10 \mathrm{~min} ; 45$ cycles of $95^{\circ} \mathrm{C}$ for $15 \mathrm{~s}, 60^{\circ} \mathrm{C}$ for $1 \mathrm{~min}$. Cycle threshold values of BAIAP3 were standardized to cycle threshold values of GAPDH.

All supplementary materials are available online at www.molmed.org.

\section{RESULTS}

\section{Generation of Baiap3 KO Mice}

Baiap3 shares the basic domain structure of other Munc13 isoforms, with two munc-homology domains flanked by two C2 domains but lacks the N-termini contained in Munc13-1, -2 and -3 (23). The murine Baiap3 gene contains 33 coding exons that span $8.7 \mathrm{~kb}$. We generated Baiap3 KO mice by homologous recombination in embryonic stem cells, replacing the first three coding exons with a neomycin selection cassette (Supplementary Figure S1A). Baiap3 KO mice are viable, fertile and indistinguishable from their wild-type (WT) littermates in the home cage. In WT brain, the expression pattern of Baiap3 protein analyzed by immunofluorescence staining largely matches the distribution of Baiap3 mRNA published in the Allen Brain Atlas. Baiap3 protein is prominently expressed throughout the hypothalamus and in the central, medial and basomedial amygdaloid nuclei, as well as in the paraventricular nucleus of the thalamus (Figure 1). Strong expression is further detected in the septum, bed nucleus of the stria terminalis, midbrain including the periaqueductal gray and inferior colliculus, and brain stem including the parabrachial nucleus and nucleus tractus solitarius (Figure 1). Baiap3 immunoreactivity appears punctate, but does not seem to localize to either glutamatergic or GABAergic pre- or postsynapses to a significant degree (Supplementary Figures S1C-G). Adult Baiap3 KO mice lack any detectable expression of Baiap3 protein by immunofluorescence and Western blot analysis (Figure 1B, Supplementary Figure S1B). Western blot analysis of brains taken from newborn Baiap3 $\mathrm{KO}$ animals revealed the presence of a weak band that most likely corresponds to Baiap3 protein expressed from a start codon present in coding exon 4; however, this putative truncated Baiap3 product is barely detectable by the age of $3 \mathrm{wks}$ and not present in adult animals (Supplementary Figure S1B).

\section{Novelty-Induced Anxiety in Baiap3 KO Mice}

The striking expression pattern of Baiap3 in the amygdala and other brain regions involved in processing fear piqued our interest, and we chose to assess whether the genetic deletion of Baiap3 led to any detectable behavioral alterations. We subjected Baiap3 KO mice and WT littermates of both sexes to a battery of standard behavioral tests (Figures 2A-L; Supplementary Figures S3A-J). In the open field, both male and female Baiap3 KO mice showed an increased latency to reach the wall upon release in the center zone (Figures 2A, B). Female but not male $\mathrm{KO}$ mice also made fewer visits to the center (Figures 2C, D) and spent significantly more time in the periphery (Figures 2E, F). When placed in a novel chamber (fear-conditioning box), both male and female $\mathrm{KO}$ mice showed an increased novelty-induced freezing response (Figures 2K, L). Taken together, these findings are indicative of a heightened novelty-induced anxiety level in Baiap3 KO animals, with a more pronounced effect noted in females. In contrast, classical tests, measuring anxi- ety in the context of an inherent conflict between a protected and a more anxiogenic area, that is, elevated plus-maze and light-dark box, did not reveal any genotype differences (Figures 2G-J). Furthermore, the distance traveled (motor activity) in open field and elevated plusmaze (Supplementary Figures S3A-D), exploratory behavior (hole board; Supplementary Figures S3E, F), motor learning and coordination (rotarod; Supplementary Figures S3G, H) and body weight (Supplementary Figures S3I, J) were not affected by genotype.

\section{$B A I A P 3$ Is a Risk Marker for Anxiety in Women}

To explore the possibility of an association of genetic variability in the human $B A I A P 3$ gene with specific biological readouts, we made use of the GRAS database of schizophrenic patients $(31,32)$. Our hypotheses regarding Baiap3/BAIAP3 function were based on the anxiety phenotype observed in Baiap3 $\mathrm{KO}$ mice and on the prominent expression of Baiap 3 in brain regions involved in processing fearful stimuli as well as in substance use disorders. We selected three single-nucleotide polymorphisms (SNPs) in the BAIAP3 gene: rs11648169 (C/G, intronic), rs2235632 (G/A, intronic) and rs1132358 (C/T, coding sequence, synonymous Asp1040Asp) (Supplementary Figure S4A) from public databases [http://www.ncbi. nlm.nih.gov/projects/SNP/; http:// browser.1000genomes.org; http:// hapmap.ncbi.nlm.nih.gov/]. The selection of SNPs was based on (i) a high minor allele frequency $(\mathrm{MAF} \geq 0.36$ ) distribution within the European Caucasian population (http:/ / www.ncbi.nlm.nih.gov/ $\mathrm{SNP} /$ ), to increase the power to detect genetic effects, and (ii) the potential for functional consequences. The last criterion could only partially be fulfilled; the exonic SNP rs1132358 (C/T, Asp1040Asp, synonymous) might potentially affect mRNA structure or stability. All SNPs fulfilled Hardy-Weinberg equilibrium criteria, both in cases and in controls $(p>$ 0.05). A construction of haplotype blocks of the three SNPs revealed a similarly 

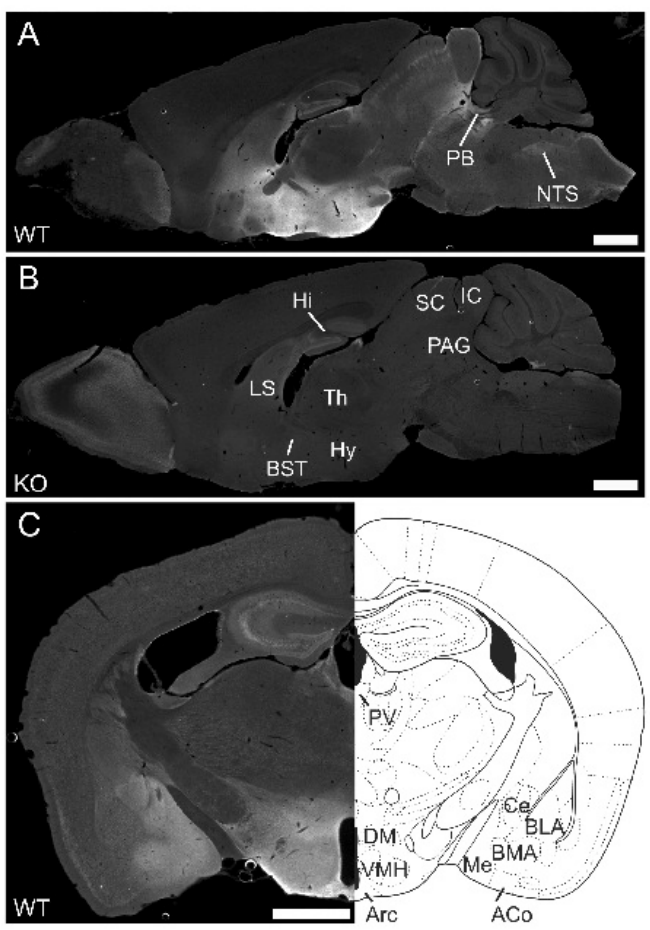

Figure 1. Immunofluorescence analysis of Baiap3 expression in mouse brain. (A) Sagittal brain section of adult Baiap3 WT mouse stained with rabbit anti-Baiap3 antibody. (B) Sagittal brain section of adult Baiap3 KO littermate showing the absence of Baiap3 immunoreactivity. Please note that the signal observed in the hippocampus of both WT and $\mathrm{KO}$ brain is a nonspecific background staining. (C) Coronal brain section of adult WT mouse stained for Baiap3 with a corresponding coronal diagram, adapted from the mouse Paxinos brain atlas (Bregma -1.46). PB, parabrachial nucleus; NTS, nucleus tractus solitarius; Hi, hippocampus; SC, superior colliculus; IC, inferior colliculus; PAG, periaqueductal gray; LS, lateral septum; Th, thalamus; Hy, hypothalamus; BST, bed nucleus of the stria terminalis; PV, paraventricular thalamic nucleus; DM, dorsomedial hypothalamic nucleus; VMH, ventromedial hypothalamic nucleus; Arc, arcuate nucleus; Ce, central amygdaloid nucleus; BLA, basolateral amygdaloid nucleus, anterior part; BMA, basomedial amygdaloid nucleus, anterior part; ME, medial amygdaloid nucleus; ACo, anterior cortical amygdaloid nucleus. Scale bars equal $1 \mathrm{~mm}$.

high degree of linkage disequilibrium between them in the GRAS sample (Supplementary Figure S4B) and in healthy controls (Supplementary Figure S4C). Case control analysis of genotype frequencies of the three SNPs did not reveal any significant differences, indicating that the selected genetic variation in BAIAP3 is not associated with schizophrenia risk (Supplementary Figure S4D). We subsequently used the PGAS approach (32) to analyze the three SNPs for association with specific phenotypic readouts rele- vant for anxiety disorders and substance use disorders. For this step, an anxiety composite score was constructed using four anxiety-relevant variables (Supplementary Figure S2), which showed a significant association with only two of the three selected SNPs (as expected because of the high linkage disequilibrium between both markers and their similar MAFs) for women but not for men (Table 1). SNP rs11648169 was excluded from further analyses, since it yielded no statistically significant effects.

\section{BAIAP3 Is a Risk Gene for Benzodiazepine Abuse in Men}

Because anxiety disorders and substance use disorders often occur together, and BAIAP 3 is expressed in brain regions relevant for emotionality and drug dependence, we also screened for a possible association between genetic variation in $B A I A P 3$ and substance use disorder. The same risk genotypes (AA for rs2235632, TT for rs1132358) that were associated with anxiety in women showed a statistically significant association with benzodiazepine use disorder in men (Table 1). Even though there was a similar tendency for women (benzodiazepine use disorder associated with $7.0 \% / 7.7 \%$ in AA/TT genotypes versus $4.7 \% / 4.6 \%$ in G/C carrier status), it did not reach statistical significance, perhaps because of the lower numbers of women than men in the GRAS sample. The genotype frequencies of rs2235632 and rs1132358 did not differ between men and women in the GRAS sample (rs2235632,

GG/AG/AA: men 25.1\%/49.1\%/25.8\%; women $28.3 \% / 46.3 \% / 25.5 \%$; rs 1132358 , CC/TC/TT: men $26.1 \% / 49.4 \% / 24.5 \%$; women $29.8 \% / 46.9 \% / 23.3 \%$ ), and neither of these two SNPs was associated with disease-related or sociodemographic control variables (Table 1).

For the purpose of an association analysis of the relevant BAIAP3 genotypes (GG/AG/AA in rs2235632 and CC/TC/TT in rs1132358) with benzodiazepine use disorder, the GRAS sample delivers an ideal, nearly experimental setting. The distribution of these genotypes among benzodiazepine users versus nonusers is highly comparable, allowing the identification of risk genotypes leading to benzodiazepine use disorder (Supplementary Table S1). Most importantly, the benzodiazepine dose was equal across all genotypes (Supplementary Table S1). Hence, the BAIAP3 risk genotypes (AA for rs2235632, TT for rs1132358) appear to confer a specific genetic risk of developing benzodiazepine use disorder given equal dose and likelihood of exposure. Interestingly, neither alcohol nor cannabis abuse were found to 
A

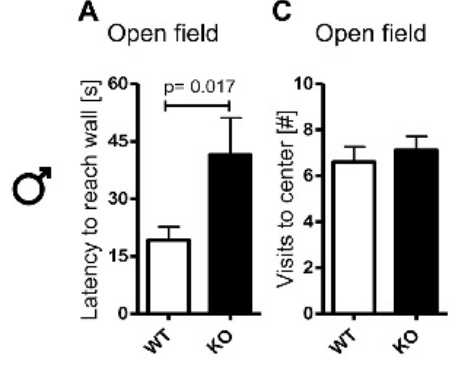

B

Open field

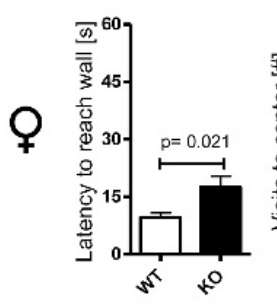

D

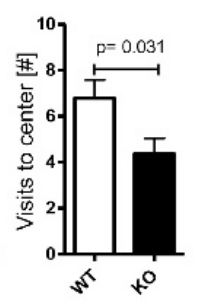

E

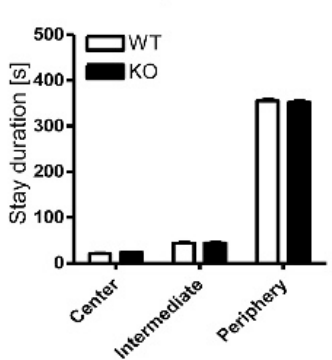

$\mathbf{F}$

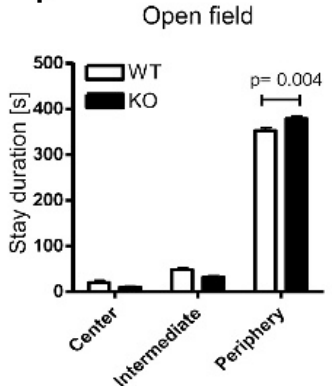

G Elevated plus-maze ${ }^{\mathrm{I}}$ Light/dark box ${ }^{\mathrm{K}}$ Freezing
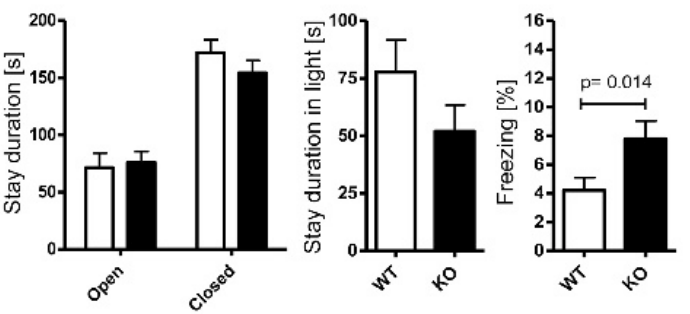

H Elevated plus-maze $\mathbf{J}_{\text {Light/dark box }}$

$\mathrm{L}_{\text {Freezing }}$
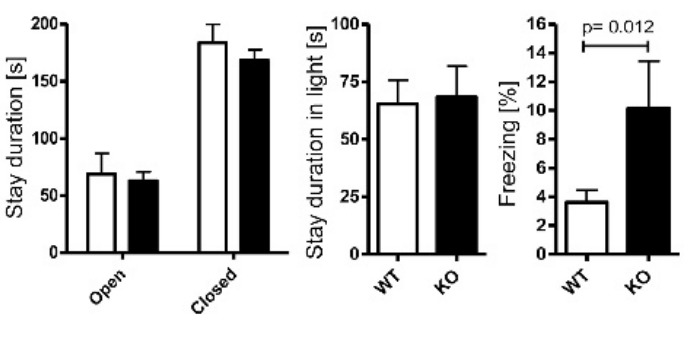

Figure 2. Anxiety phenotype in Baiap3 KO mice. (A-F) Open field parameters. (A, B) The latency to reach the wall of the open field was significantly increased in Baiap3 KO mice of both sexes, whereas visits to the center (C, D) and stay in the periphery (E, F) revealed anxiety-like behavior only in females. Elevated plus-maze $(G, H)$ and light-dark box $(l, J)$ revealed no genotype-dependent differences in either sex. (K, L) As readout of unspecific novelty-related anxiety, a higher freezing response was found in male as well as female Baiap3 KO mice. Numbers tested: males, $\mathrm{WT}=16-25, \mathrm{KO}=16-25$; females, $\mathrm{WT}=18-23, \mathrm{KO}=10-28$. Mann-Whitney $U$ test $(\mathrm{A}-\mathrm{D}, \mathrm{I}-\mathrm{L})$ and two-way ANOVA $(E-H)$, including Bonferroni testing, were applied. Means \pm SEM are presented.

be associated with the two SNPs, pointing to a specific benzodiazepine link with the selected BAIAP3 genotypes (Table 1).

To determine whether the identified risk genotypes are associated with altered expression of BAIAP3, we analyzed the mRNA levels of BAIAP3 in PBMCs obtained from 121 subjects by qRT-PCR. We found a statistically significant association of the BAIAP3 risk genotypes (AA for rs2235632, TT for rs1132358) with lower BAIAP3 mRNA levels in PBMCs of male individuals, which is at least partially comparable to a gene dose reduction or $\mathrm{KO}$ situation. This result is not found in women, possibly because of the lower numbers available for analysis (Supplementary Figure S5). However, these findings could also support the interpretation that the effects of BAIAP3 risk alleles are gender specific.

\section{Male Baiap3 KO Mice Show Faster Development of Tolerance to Benzodiazepines}

On the basis of the identification of human BAIAP3 risk genotypes for benzodiazepine abuse in male patients, we tested Baiap3 $\mathrm{KO}$ and WT littermates of both sexes in experimental paradigms of chronic benzodiazepine administration to assess the development of tolerance, dependence and withdrawal (Figure 3A). The baseline performance of each mouse in the rotarod test was established on two consecutive days of rotarod training. No significant genotype-dependent differences were detected in baseline performance (Supplementary Figures S3G, $\mathrm{H})$. Benzodiazepine dependence in Baiap $3 \mathrm{KO}$ and WT mice of both sexes was then induced with daily diazepam injections ( $5 \mathrm{mg} / \mathrm{kg}$ IP) for 10 consecu- tive days. To monitor the development of tolerance to diazepam, motor performance on rotarod at $30 \mathrm{~min}$ after each injection was evaluated over the first $7 \mathrm{~d}$ of diazepam treatment. Rapid development of tolerance to daily diazepam injections was apparent in both sexes and genotypes by an increase of the latency of falling from rotarod over the course of $7 \mathrm{~d}$ (Figures 3B, C). Here, male Baiap3 KO mice performed significantly better than their WT littermates (Figure 3B), whereas no such difference was detected for females (Figure 3C). Thus, male Baiap3 KO mice show faster development of tolerance to diazepam.

\section{Baiap3 KO Mice Have an Increased Seizure Propensity}

To evaluate whether Baiap3 genotype would affect the propensity for di- 


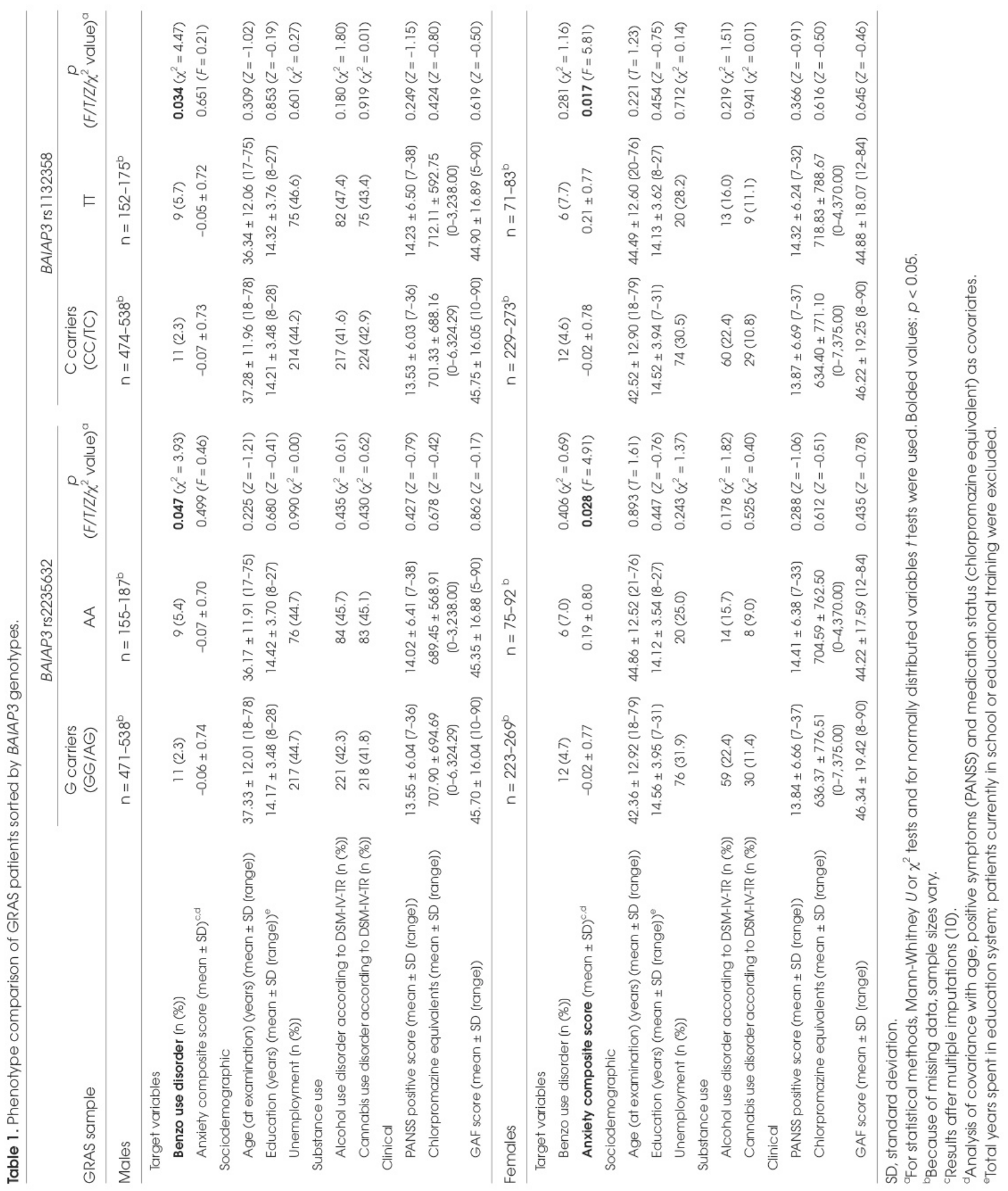




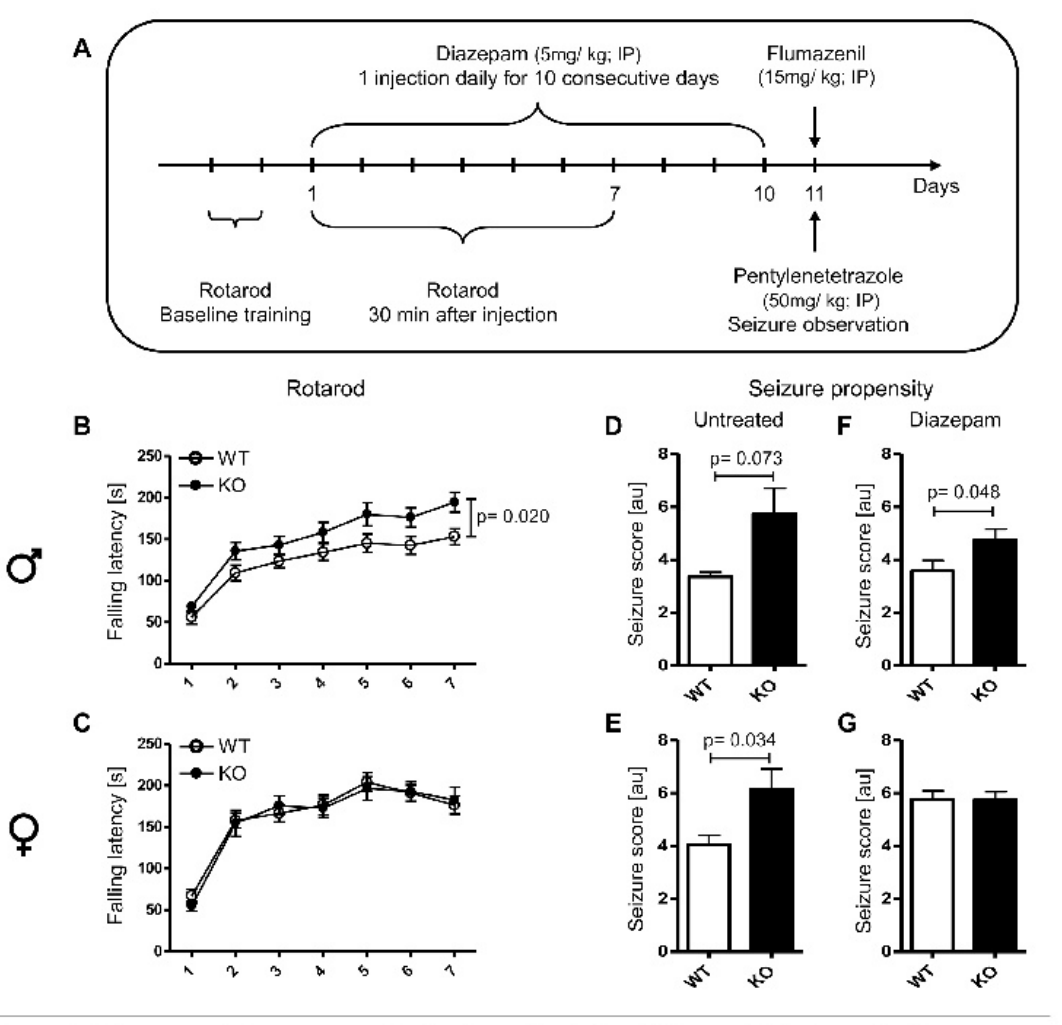

Figure 3. Diazepam tolerance and withdrawal in Baiap3 KO and WT mice. (A) Experimental design scheme. (B) Male diazepam-treated Baiap3 KO mice showed significantly faster improvement of performance on the rotarod, consistent with a more rapid development of tolerance to diazepam. (C) Rotarod performance of female mice was comparable between WT and KO. (D, E) Diazepam-naive Baiap3 KO mice display a higher PTZ-induced seizure propensity compared with WT (significant in females, strong tendency in males). (F, G) Flumazenil-induced diazepam withdrawal does not further increase PTZinduced seizure propensity in Baiap3 KO mice. Seizure propensity of female mice became comparable between genotypes, pointing to a ceiling effect. Numbers tested: males, $W T=25, K O=25$; females, $W T=21, K O=23$, except for $(D)$ and $(E)$, where males, $W T=7$, $\mathrm{KO}=7$; females, $\mathrm{WT}=8 ; \mathrm{KO}=10$. Mann-Whitney $U$ test $(\mathrm{D}-\mathrm{G})$ and two-way repeatedmeasures ANOVA (B, C), including Bonferroni, testing applied. Means \pm SEM are presented.

azepam withdrawal-related seizures, the susceptibility to PTZ-induced seizures was first evaluated in diazepam-naive mice. The seizure response of Baiap3 $\mathrm{KO}$ mice of both sexes to PTZ (50 mg/ kg IP) was higher than that in WT animals, with the difference just failing to reach significance in males (Figures 3D, E). To assess the effect of genotype on benzodiazepine withdrawal, the diazepam antagonist flumazenil (15 mg/kg IP) was injected on d 11, after $10 \mathrm{~d}$ of daily diazepam treatment, immediately followed by PTZ injection ( $50 \mathrm{mg} / \mathrm{kg} \mathrm{IP}$ ) to trigger withdrawal seizures (Figure 3A). Upon flumazenil-induced diazepam withdrawal, the response to PTZ in male Baiap3 KO and WT mice did not differ appreciably from the one found in diazepam-naive mice of both genotypes (Figure 3F). In contrast, the genotypedependent differences in diazepam-naive females regarding seizure scores disappeared under conditions of diazepam withdrawal (Figure 3G), which could be explained by a ceiling effect. Thus, fe- male and male Baiap3 $\mathrm{KO}$ mice are more seizure-prone than their WT littermates, and this propensity is not further increased by benzodiazepine withdrawal.

\section{Drug Self-administration and Basic Behaviors Do Not Differ between Baiap3 Genotypes upon Chronic Addiction}

To assess whether Baiap3 KO mice, once addicted, would also be more likely to orally self-administer benzodiazepines, we performed an experiment on chronic midazolam addiction, where self-application was assessed after forced long-term exposure to escalating doses of midazolam (Supplementary Figure S6). We detected no genotype or gender differences in the clear preference for midazolam. Moreover, no genotype effects on body weight or basic behavior in the chronically addicted state were noted (Supplementary Figure S6). These data indicate that the Baiap3 genotype gender specifically affects the development of tolerance, that is, drug abuse at an early stage. In chronic addiction, genotype effects are no longer detectable.

\section{Lack of Homeostatic Adaptation to Diazepam in Baiap3 KO Hypothalamus Slices}

One hypothesis regarding predisposition to the development of addiction at the cellular level is an altered response to the addiction-inducing substance and its withdrawal. Because Baiap3 KO mice showed an increased seizure propensity and an altered development of tolerance to diazepam, we investigated whether lack of Baiap3 leads to a measurably altered response to diazepam treatment and withdrawal in neurons in vitro. Because Baiap3 expression is highest in the hypothalamus, we cultured organotypic hypothalamus slices prepared from male P5/P6 Baiap3 KO and WT animals in the presence of either $10 \mu \mathrm{mol} / \mathrm{L}$ diazepam or vehicle (DMSO) and recorded from neurons in the ventromedial hypothalamus in the presence of the diazepam antagonist flumazenil to 
mimic diazepam withdrawal conditions in vitro. We hypothesized that diazepam treatment would lead to a homeostatic adaptation in the $\mathrm{GABA}_{\mathrm{A}} \mathrm{R}$-mediated mIPSCs (Figure 4A) that would become apparent under diazepam withdrawal conditions. Although we observed no diazepam treatment-dependent differences that reached statistical significance, there was a significant genotypedependent effect under diazepam withdrawal conditions. Here, WT mIPSC amplitudes were $27 \%$ smaller (Figure 4B) and rise times 13\% longer than in $\mathrm{KO}$ neurons (Figure 4C), which is suggestive of a homeostatic adaptation to diazepam treatment in WT but not in KO slices. No significant differences in MIPSC decay times and frequencies were observed (Supplementary Table S2). Because the sudden withdrawal of diazepam should lead to an increase in overall network activity, we recorded action potential (AP) frequencies in ventromedial hypothalamus slices in the presence of flumazenil. Surprisingly, KO slices already showed significantly higher AP frequencies than WT slices under control conditions, with no further increase under diazepam withdrawal conditions. By contrast, in WT slices, we observed a significant increase in AP frequency under diazepam withdrawal conditions compared with vehicle-treated WT slices (Figure 4D). There was no significant difference in the resting membrane potentials (Figure $4 \mathrm{E}), \mathrm{AP}$ rise times, decay times and half-widths (Supplementary Table S2). IPSCs were recorded in the same cells at a holding potential of $-20 \mathrm{mV}$ to be able to isolate spontaneous $\mathrm{GABA}_{\mathrm{A}} \mathrm{R}$ mediated currents without drug application. In WT slices, we observed a significant effect of diazepam withdrawal, with an increase in IPSC amplitude and frequency compared with vehicletreated WT slices (Figures 4F, G), which is in keeping with the overall higher firing rate and which was not apparent in KO slices. In summary, these data show that neurons in Baiap3 KO hypothalamus slices have higher AP firing rates,

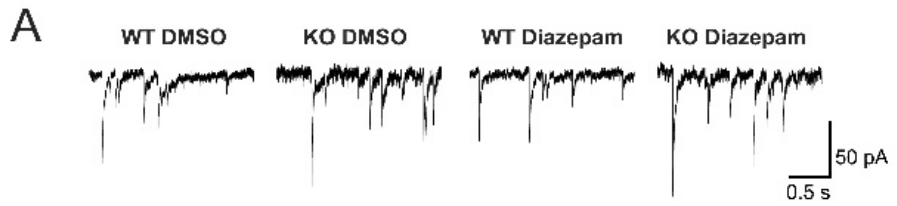

B mIPSc Amplitude

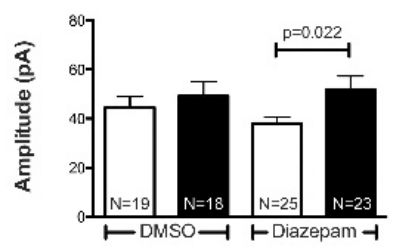

D Action Potential Frequency

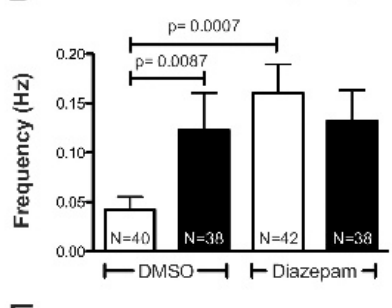

$\mathrm{F}$

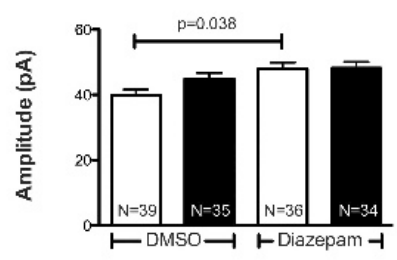

C

mIPSC Rise Time

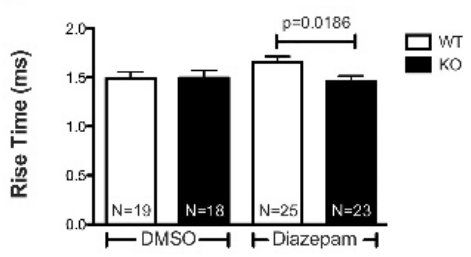

E Resting Membrane Potential
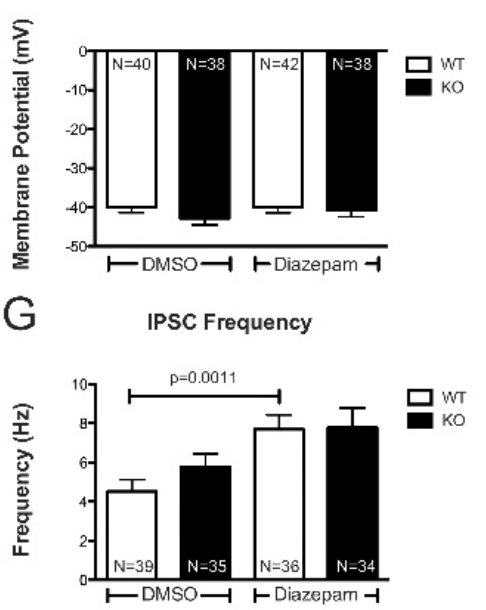

Figure 4. Increased basal network activity and lack of homeostatic adaptation to diazepam treatment in Baiap3 KO hypothalamus slices. (A) Sample traces of mIPSC recordings from WT and KO hypothalamus slices that were cultured in the presence of diazepam or under vehicle control conditions with DMSO. Under diazepam withdrawal conditions, Baiap3 WT mIPSC amplitudes were significantly smaller than in KO slices (B), and WT MIPSC rise times were longer than in KO slices (C). (D) Baiap3 WT slices showed an increase in AP frequency in response to diazepam withdrawal when compared with DMSO-treated WT slices, whereas no such increase was apparent for Baiap3 KO slices, which already showed an increased AP frequency under DMSO control conditions when compared with WT slices. (E) The resting membrane potential was not affected by experimental condition or Baiap3 genotype. IPSC amplitudes (F) and IPSC frequencies (G) were increased in Baiap3 WT slices under diazepam withdrawal compared with DMSO-treated WT slices. Mann-Whitney $U$ test was used for AP and IPSC frequencies; Student $t$ test was used for all other parameters. Means \pm SEM are presented.

likely consistent with the higher seizure propensity found in vivo, and that Baiap3 KO slices show no obvious homeostatic adaptation to diazepam treatment and withdrawal.

\section{DISCUSSION}

In this study, we identify two human $B A I A P 3$ risk genotypes that are associated with anxiety in women and benzodiazepine use disorder in men. We fur- 
ther show that Baiap 3 deficiency in mice leads to (i) elevated seizure propensity; (ii) increased anxiety in both genders, with a more pronounced effect in females; and (iii) a faster development of tolerance to benzodiazepines in male mice. In vitro analysis of hypothalamic slices revealed an increase in neuronal baseline activity in the absence of Baiap3. Withdrawal from chronic benzodiazepine application in vitro results in a genotype-specific response pattern.

To the best of our knowledge, no other genetic risk marker that is associated with anxiety and benzodiazepine abuse has been reported to date. We are aware that, pending replication in nonschizophrenic individuals, we cannot be sure that our findings can be applied to the general population. In spite of this limitation, our findings suggest a role for $B A I A P 3$ and potential interaction partners in the development of anxiety and drug dependence.

Unfortunately, similar data from samples of equally well-phenotyped healthy individuals or even other disease groups are not available. This is particularly true with regard to benzodiazepine abuse, since short-term exposure is a primary goal of controlled and medically surveyed indications. Even looking at other rare situations of long-term exposure (for example, intractable epilepsies), a homogeneous sample comparable to the GRAS collection would be difficult to imagine. In the GRAS sample, there are no differences between BAIAP3 genotypes regarding benzodiazepine exposure or daily dose (in case of exposure). This constellation allowed us to analyze the specific genetic risk of developing benzodiazepine use disorder in a setting close to an experimental condition.

Importantly, the present study was purely hypothesis-driven. Our hypotheses for performing a human phenotypebased genetic association study of $B A I A P 3$ were based on the anxiety phenotype we observed during basic behavioral characterization of Baiap3 $\mathrm{KO}$ mice as well as on the distinctive Baiap3 ex- pression pattern in brain, which includes regions relevant for addictive behaviors. We find that in humans, female carriers of the homozygous BAIAP3 risk genotypes (AA for SNP rs2235632; TT for SNP rs1132358) are more likely to meet criteria for an anxiety disorder, whereas male carriers of the same risk genotypes are more likely to fulfill criteria for benzodiazepine use disorder. Neither SNP was associated with schizophrenia in our case control analysis. Furthermore, no associations with substance use disorder other than benzodiazepine use disorder were observed. In general, both genetic linkage and candidate gene studies suffer from lack of replicability (12). However, in our study, the parallel identification of a gender-specific association of BAIAP3/Baiap3 with anxiety and an altered response to benzodiazepines in both mice and men, lends strong support to a causal link between BAIAP3 and the observed phenotypes.

As for other genetic variations associated with anxiety disorders $(11,12)$ or substance use disorders (13-15), the impact of BAIAP3 genotypes on anxiety disorders or benzodiazepine use disorder is likely to be small. However, the observed effects and their gender specificity (across two species) are intriguing. While we currently have no mechanistic insight into this gender specificity, part of the explanation may lie in the fact that Baiap3 is expressed in sexually dimorphic brain regions such as the hypothalamus, amygdala and the bed nucleus of the stria terminalis $(38,39)$.

Given the higher prevalence of both anxiety disorders and benzodiazepine use disorder in women $(9,40)$ the present findings were surprising at first glance, but the similarity of gender differences in mice and humans underlines their significance, encouraging follow-up work on this gender effect. Admittedly, the gender effects in humans may ultimately turn out to be less prominent, since the total number of individuals with benzodiazepine abuse in the GRAS sample is low, resulting in moderate significance levels only for men. It cannot be excluded that, in a larger sample, an association of benzodiazepine use disorder with the genotypes investigated here might reach significance for women as well. Furthermore, research focusing on gender differences and social desirability in self-reported anxiety suggests an underreporting of fear and distress in men (41-43). Therefore, our use of three self-reported measures in the calculation of the anxiety composite score might partly explain the lack of association of the BAIAP3 risk genotypes with anxiety in men. Nevertheless, gender differences in BAIAP3/Baiap3 genotype-phenotype associations most likely exist and are worth pursuing.

Benzodiazepines are positive allosteric modulators of $\mathrm{GABA}_{\mathrm{A}} \mathrm{R}$ and thus enhancers of inhibitory GABAergic neurotransmission. Their sedative, anti-convulsive and amnesic effects are largely mediated by the $\mathrm{GABA}_{\mathrm{A}} R \alpha 1$ subunit, the anxiolytic effect by the $\alpha 2$ subunit and muscle relaxation by $\alpha 2, \alpha 3$ and $\alpha 5$ subunits (44). To date, no specific risk association of these obvious candidate genes has been identified. At present we have no evidence that would suggest that Baiap3 interacts with $\mathrm{GABA}_{\mathrm{A}} \mathrm{R}$ subunits. However, the increased seizure propensity observed in Baiap $3 \mathrm{KO}$ mice of both sexes, which is already apparent without prior diazepam treatment and withdrawal, is indicative of an altered balance of excitatory and inhibitory systems. Our comparison of neuronal firing rates in hypothalamus slices under baseline and diazepam withdrawal conditions uncovered an increase in basal network activity in the absence of Baiap3. This finding was unexpected, and although presently limited to the hypothalamus, is consistent with the increased seizure propensity observed in vivo. Even though we do not know whether the seizures observed in our PTZ-induction model originate in the subcortical regions that express Baiap3, subcortical epileptogenesis with origins in the hypothalamus is a feature seen in hypothalamic hamartomas (45), and the amygdala, which also expresses Baiap3, is known to play a key role in epileptogenesis (46). 
Interestingly, the human BAIAP3 gene is located on chromosome 16p13.3, which has been linked to electroencephalographic traits of idiopathic epilepsy syndromes $(47,48)$. We would thus argue that further investigation of BAIAP3 as a candidate gene for epilepsy-related phenotypes is warranted. Because we found that Baiap3 did not colocalize with markers of GABAergic or glutamatergic preand postsynapses to a significant degree (Supplementary Figures S1C-G), the increased seizure propensity in Baiap3 $\mathrm{KO}$ mice of both sexes and the altered response to benzodiazepines in males is unlikely to be due to a direct effect of Baiap3 at GABAergic or glutamatergic synapses.

The neuronal circuitry underlying the addictive properties of benzodiazepines is less well understood than their molecular mechanism of action. Unlike many other addictive substances, benzodiazepines do not appear to increase dopamine levels in the nucleus accumbens (49-51), although electrophysiological studies suggest that benzodiazepines increase firing of dopaminergic neurons in the VTA through disinhibition of these neurons via inhibition of nearby inhibitory interneurons $(52,53)$. Additional mechanisms, such as neuroendocrine responses to benzodiazepine treatment, may play a critical role in the development of benzodiazepine use disorder (54). Furthermore, because expression of Baiap3 in both the VTA and in the nucleus accumbens is low (Allen Brain Atlas), a direct effect of Baiap3 on the mesolimbic dopamine pathway does not appear be the most likely explanation for the observed interaction between Baiap3 genotypes and the response to benzodiazepines. Instead, our findings support the interpretation that the altered response to benzodiazepines could be a consequence of a local or global change in neuronal excitability. Because all other members of the Munc13 protein family have been shown to be regulators of SNARE-mediated exocytosis $(25,29)$, Baiap3 may regulate the release of one or more modulatory neurotransmitters or neuropeptides that influence the balance between GABAergic and glutamatergic neurotransmission. Baiap3 immuoreactivity appears punctate (Supplementary Figures S1C-G) and may localize to peptidergic release sites, some of which may also contain VGLUT2 or Viaat. Although we presently cannot exclude the possibility that Baiap3 might have a postsynaptic function, given what is know about the function of all other members of the Munc13 protein family, we think that a pre-synaptic function is more likely. We can furthermore not exclude the possibility that alterations in the hypothalamicpituitary-adrenal axis may play a role in the anxiety phenotype or the altered response to benzodiazepines seen in Baiap3 $\mathrm{KO}$ mice. We are currently investigating whether Baiap3 is involved in regulating exocytosis of dense core vesicles and/or intracellular trafficking events that could influence neuropeptide release or extrasynaptic $\mathrm{GABA}_{\mathrm{A}}$ Rs.

\section{CONCLUSION}

To conclude, BAIAP3 had not previously been considered a candidate gene for either psychiatric disorders or epilepsy. Our study links BAIAP3/Baiap3 genotypes to anxiety and an altered response to benzodiazepines in both mice and men and thus strongly argues for an involvement of BAIAP3 in these neuropsychiatrically relevant phenotypes. The identification of human genetic variations that influence the risk for the development of pathological phenotypes as well as the response to pharmacological treatments may pave the way for more efficient treatments with fewer side effects. Rodent models are usually only imperfect representations of human psychiatric conditions; however, the simultaneous identification of Baiap3 as a biomarker for anxiety and the response to benzodiazepines in mouse and humans suggests that Baiap3 $\mathrm{KO}$ mice will be a valuable tool in further elucidating the genetic, physiological and neuroanatomical underpinnings of anxiety disorders and benzodiazepine use disorder.

\section{ACKNOWLEDGMENTS}

We are indebted to all patients for their participation in the GRAS study and all collaborating GRAS centers for their support. We are grateful to all colleagues who contributed to the GRAS data collection. We would also like to thank Astrid Zeuch, Astrid Ohle and the members of the DNA sequencing core facility for excellent technical assistance. This work was supported by the Max Planck Society, the MaxPlanck-Förderstiftung, and the DFG Center for Nanoscale Microscopy and Molecular Physiology of the Brain.

\section{DISCLOSURE}

The authors declare that they have no competing interests as defined by Molecular Medicine, or other interests that might be perceived to influence the results and discussion reported in this paper.

\section{REFERENCES}

1. Kessler RC, et al. (2005) Lifetime prevalence and age-of-onset distributions of DSM-IV disorders in the National Comorbidity Survey Replication. Arch. Gen. Psychiatry. 62:593-602.

2. Pasche S. (2012) Exploring the comorbidity of anxiety and substance use disorders. Curr. Psychiatry Rep. 14:176-81.

3. Swendsen J, et al. (2010) Mental disorders as risk factors for substance use, abuse and dependence: results from the 10-year follow-up of the National Comorbidity Survey. Addiction. 105:1117-28.

4. Conway KP, Compton W, Stinson FS, Grant BF. (2006) Lifetime comorbidity of DSM-IV mood and anxiety disorders and specific drug use disorders: results from the National Epidemiologic Survey on Alcohol and Related Conditions. J. Clin. Psychiatry. 67:247-57.

5. Myrick H, Brady K. (2003) Current review of the comorbidity of affective, anxiety, and substance use disorders. Curr. Opin. Psychiatry. 16:261-70.

6. Bandelow B, et al. (2012) Guidelines for the pharmacological treatment of anxiety disorders, obsessive-compulsive disorder and posttraumatic stress disorder in primary care. Int. J. Psychiatry Clin. Pract. 16:77-84.

7. Baker AL, Thornton LK, Hiles S, Hides L, Lubman DI. (2012) Psychological interventions for alcohol misuse among people with co-occurring depression or anxiety disorders: a systematic review. J. Affect. Disord. 139:217-29.

8. Hettema JM, Neale MC, Kendler KS. (2001) A review and meta-analysis of the genetic epidemiology of anxiety disorders. Am. J. Psychiatry. 158:1568-78.

9. Hettema JM, Prescott CA, Myers JM, Neale MC, 


\section{Appendix}

Kendler KS. (2005) The structure of genetic and environmental risk factors for anxiety disorders in men and women. Arch. Gen. Psychiatry. 62:182-9.

10. Ducci F, Goldman D. (2012) The genetic basis of addictive disorders. Psychiatr. Clin. North Am. 35:495-519.

11. Hovatta I, Barlow C. (2008) Molecular genetics of anxiety in mice and men. Ann. Med. 40:92-109.

12. Hamilton SP. (2009) Linkage and association studies of anxiety disorders. Depress. Anxiety. 26:976-83.

13. Wang JC, Kapoor M, Goate AM. (2012) The genetics of substance dependence. Annu. Rev. Genomics Hum. Genet. 13:241-61.

14. Gelernter J, Kranzler HR. (2010) Genetics of drug dependence. Dialogues Clin. Neurosci. 12:77-84.

15. Buckland PR. (2008) Will we ever find the genes for addiction? Addiction. 103:1768-76.

16. Marmorstein NR. (2012) Anxiety disorders and substance use disorders: different associations by anxiety disorder. J. Anxiety Disord. 26:88-94.

17. DeMartini KS, Carey KB. (2011) The role of anxiety sensitivity and drinking motives in predicting alcohol use: a critical review. Clin. Psychol. Rev. 31:169-77.

18. Zavos HM, Gregory AM, Eley TC. (2012) Longitudinal genetic analysis of anxiety sensitivity. Dev. Psychol. 48:204-12.

19. Kushner MG, Thuras P, Abrams K, Brekke M, Stritar L. (2001) Anxiety mediates the association between anxiety sensitivity and coping-related drinking motives in alcoholism treatment patients. Addict. Behav. 26:869-85.

20. Baldwin DS, Allgulander C, Bandelow B, Ferre F, Pallanti S. (2012) An international survey of reported prescribing practice in the treatment of patients with generalised anxiety disorder. World J. Biol. Psychiatry. 13:510-6.

21. Canteras NS, Resstel LB, Bertoglio LJ, Carobrez Ade P, Guimaraes FS. (2010) Neuroanatomy of anxiety. Curr. Top. Behav. Neurosci. 2:77-96.

22. Gratacos M, et al. (2007) Candidate genes for panic disorder: insight from human and mouse genetic studies. Genes Brain Behav. 6 (Suppl. 1):2-23.

23. Koch H, Hofmann K, Brose N. (2000) Definition of Munc13-homology-domains and characterization of a novel ubiquitously expressed Munc13 isoform. Biochem. J. 349:247-53.

24. Shiratsuchi T, et al. (1998) Cloning and characterization of BAP3 (BAI-associated protein 3), a C2 domain-containing protein that interacts with BAI1. Biochem. Biophys. Res. Commun. 251:158-65.

25. Varoqueaux F, et al. (2002) Total arrest of spontaneous and evoked synaptic transmission but normal synaptogenesis in the absence of Munc13-mediated vesicle priming. Proc. Natl. Acad. Sci. U. S. A. 99:9037-42.

26. Gorman JM, Kent JM, Sullivan GM, Coplan JD. (2000) Neuroanatomical hypothesis of panic disorder, revised. Am. J. Psychiatry. 157:493-505.

27. Gross CT, Canteras NS. (2012) The many paths to fear. Nat. Rev. Neurosci. 13:651-8.

28. Wojcik SM, Brose N. (2007) Regulation of mem- brane fusion in synaptic excitation-secretion coupling: speed and accuracy matter. Neuron. 55:11-24.

29. Feldmann J, et al. (2003) Munc13-4 is essential for cytolytic granules fusion and is mutated in a form of familial hemophagocytic lymphohistiocytosis (FHL3). Cell. 115:461-73.

30. Shirakawa R, et al. (2004) Munc13-4 is a GTPRab27-binding protein regulating dense core granule secretion in platelets. J. Biol. Chem. 279:10730-7

31. Begemann M, et al. (2010) Modification of cognitive performance in schizophrenia by complexin 2 gene polymorphisms. Arch. Gen. Psychiatry. 67:879-88.

32. Ribbe K, et al. (2010) The cross-sectional GRAS sample: a comprehensive phenotypical data collection of schizophrenic patients. BMC Psychiatry. 10:91.

33. Ferraro TN, et al. (1999) Mapping loci for pentylenetetrazol-induced seizure susceptibility in mice. J. Neurosci. 19:6733-9.

34. American Psychiatric Association (APA). (2000) Diagnostic and Statistical Manual of Mental Disorders: DSM-IV-TR. 4th ed., text revision. Washington (DC): APA. 992 pp

35. Kay SR, Fiszbein A, Opler LA. (1987) The positive and negative syndrome scale (PANSS) for schizophrenia. Schizophr. Bull. 13:261-76.

36. Cronbach LJ. (1951) Coefficient alpha and the internal structure of tests. Psychometrika. 16:297-334

37. Rubin DB. (1987) Multiple Imputation for Non-Response in Surveys. New York: John Wiley \& Sons.

38. Stefanova N, Ovtscharoff W. (2000) Sexual dimorphism of the bed nucleus of the stria terminalis and the amygdala. Adv. Anat. Embryol. Cell Biol. 158:III-X, 1-78.

39. Qureshi IA, Mehler MF. (2010) Genetic and epigenetic underpinnings of sex differences in the brain and in neurological and psychiatric disease susceptibility. Prog. Brain Res. 186:77-95.

40. Neutel CI. (2005) The epidemiology of long-term benzodiazepine use. Int. Rev. Psychiatry. 17:189-97.

41. Pierce KA, Kirkpatrick DR. (1992) Do men lie on fear surveys? Behav. Res. Ther. 30:415-8.

42. McLean CP, Anderson ER. (2009) Brave men and timid women? A review of the gender differences in fear and anxiety. Clin. Psychol. Rev. 29:496-505.

43. Stoyanova M, Hope DA. (2012) Gender, gender roles, and anxiety: perceived confirmability of self report, behavioral avoidance, and physiological reactivity. J. Anxiety Disord. 26:206-14.

44. Tan KR, Rudolph U, Luscher C. (2011) Hooked on benzodiazepines: GABAA receptor subtypes and addiction. Trends Neurosci. 34:188-97.

45. Fenoglio KA, et al. (2007) Hypothalamic hamartoma: basic mechanisms of intrinsic epileptogenesis. Semin. Pediatr. Neurol. 14:51-9.

46. Aroniadou-Anderjaska V, Fritsch B, Qashu F, Braga MF. (2008) Pathology and pathophysiology of the amygdala in epileptogenesis and epilepsy. Epilepsy Res. 78:102-16.

47. Pinto D, et al. (2005) Genome-wide linkage scan of epilepsy-related photoparoxysmal electroencephalographic response: evidence for linkage on chromosomes 7q32 and 16p13. Hum. Mol. Genet. 14:171-8.

48. de Kovel CG, et al. (2010) Whole-genome linkage scan for epilepsy-related photosensitivity: a mega-analysis. Epilepsy Res. 89:286-94.

49. Zetterstrom T, Fillenz M. (1990) Local administration of flurazepam has different effects on dopamine release in striatum and nucleus accumbens: a microdialysis study. Neuropharmacology. 29:129-34

50. Invernizzi R, Pozzi L, Samanin R. (1991) Release of dopamine is reduced by diazepam more in the nucleus accumbens than in the caudate nucleus of conscious rats. Neuropharmacology. 30:575-8.

51. Finlay JM, Damsma G, Fibiger HC. (1992) Benzodiazepine-induced decreases in extracellular concentrations of dopamine in the nucleus accumbens after acute and repeated administration. Psychopharmacology (Berl.). 106:202-8.

52. Tan KR, et al. (2010) Neural bases for addictive properties of benzodiazepines. Nature. 463:769-74.

53. O'Brien DP, White FJ. (1987) Inhibition of nondopamine cells in the ventral tegmental area by benzodiazepines: relationship to A10 dopamine cell activity. Eur. J. Pharmacol. 142:343-54.

54. Heberlein A, Bleich S, Kornhuber J, Hillemacher T. (2008) Neuroendocrine pathways in benzodiazepine dependence: new targets for research and therapy. Hum. Psychopharmacol. 23:171-81. 